\title{
High-resolution spectroscopy of low-mass stars
}

\author{
Dissertation
}

zur Erlangung des mathematisch-naturwissenschaftlichen Doktorgrades

"Doctor rerum naturalium"

der Georg-August-Universität Göttingen

im Promotionsprogramm GAUSS

der Georg-August University School of Science (GAUSS)

vorgelegt von

Ulf Seemann

aus

Bremen

Göttingen, 2014 
Betreuungsausschuss

Prof. Dr. Ansgar Reiners, Stellare Astrophysik, Institut für Astrophysik

Prof. Dr. Stefan Dreizler, Stellare Astrophysik, Institut für Astrophysik

Prof. Dr. Peter Hauschildt, Numerische Astrophysik, Hamburger Sternwarte

Dr. Hans Ulrich Käufl, Instrumentierung, European Southern Observatory

Mitglieder der Prüfungskommission

Referent/in: Prof. Dr. Ansgar Reiners, Stellare Astrophysik, Institut für Astrophysik

Korreferent/in: Prof. Dr. Stefan Dreizler, Stellare Astrophysik, Institut für Astrophysik ggf. 2. Korreferent/in: ...

Weitere Mitglieder der Prüfungskommission:

Prof. Dr. Artie P. Hatzes, Exoplaneten, Thüringer Landessternwarte Tautenburg

Prof. Dr. Wolfgang Glatzel, Theoretische Astrophysik, Institut für Astrophysik

Prof. Dr. Wolfram Kollatschny, Extragalaktische Astrophysik, Institut für Astrophysik

Prof. Dr. Jürgen Schmitt, Stellare Astrophysik, Hamburger Sternwarte

Tag der mündlichen Prüfung: 
To my parents 

"Die Astronomie ist vielleicht diejenige Wissenschaft, worin das wenigste durch Zufall entdeckt worden ist, wo der menschliche Verstand in seiner ganzen Größe erscheint und wo der Mensch am besten lernen kann, wie klein er ist."

Georg Christoph Lichtenberg (1742 - 1799) 



\section{Contents}

Contents

vii

List of Figures $x$

List of Tables $\quad$ xiii

List of Acronyms xiv

Abstract $\quad$ xix

$\begin{array}{ll}\text { Preface } & x x i\end{array}$

I. Activity and Rotation in the Hyades 1

1. Abstract 3

2. Introduction 5

3. Observations 9

3.1. Defining the sample . . . . . . . . . . . . . . . . 9

3.2. Observations . . . . . . . . . . . . . . . . . . 11

3.3. Data reduction . . . . . . . . . . . . . . . . . . 12

4. Analysis 21

4.1. Spectral typing . . . . . . . . . . . . . . . . . . . 21

4.2. $\mathrm{H} \alpha$ linestrength . . . . . . . . . . . . . . . . . . . . . . 22

4.3. Coronal X-ray emission . . . . . . . . . . . . . . . . . . . 24

4.4. Rotational velocities . . . . . . . . . . . . . . . . 25

4.4.1. Crosscorrelation procedure . . . . . . . . . . . . . . 27

4.4.2. Template selection . . . . . . . . . . . . . . 27

4.4.3. Template matching. . . . . . . . . . . . . . . . 28

4.4.4. Error estimation . . . . . . . . . . . . . . . 28

4.5. Binary star contamination . . . . . . . . . . . . . . . . 29

4.5.1. Photometric binaries . . . . . . . . . . . . . . . . . 30

4.5.2. Spectroscopic binaries . . . . . . . . . . . . . . . . 31

5. Results 33

5.1. Multiplicity . . . . . . . . . . . . . . . . . . 33

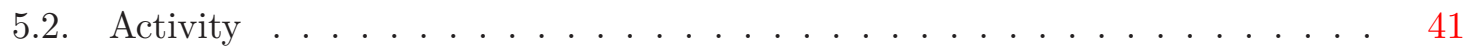

5.2.1. Chromospheric activity . . . . . . . . . . . . . . 42

$5.2 .2 . \quad$ Coronal activity . . . . . . . . . . . . . . . . . . 44 
5.2.3. Activity fraction . . . . . . . . . . . . . . . . . 47

5.3. Rotational velocities ..................... . 50

5.3.1. Fraction of rotators . . . . . . . . . . . . . . 51

5.3.2. Comparison to previous results . . . . . . . . . . . . . 51

5.3.3. Rotation-Activity relation ................. 53

6. Discussion $\quad 61$

6.1. Comparison to field stars . . . . . . . . . . . . . . . . . . . 61

6.2. Comparison to rotation periods ................... 64

6.3. Rotation and activity in binaries .................. 65

6.4. H $\alpha$ inactive rotators . . . . . . . . . . . . . . . . . . . . 66

6.5. Is the rotation limit linked to the fully convective boundary? . . . . . . . 67

$\begin{array}{lr}\text { 7. Summary } & 69\end{array}$

$\begin{array}{lr}\text { Appendices } & 71\end{array}$

A. Results on rotation and activity in the Hyades $\quad 72$

II. NAGICS-A novel absorption gas cell design for the CRIRES spectrograph 81

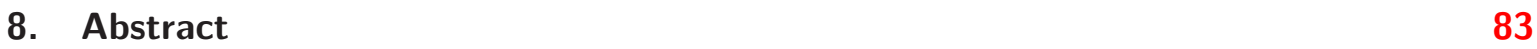

9. Introduction $\quad 85$

9.1. Motivation .......................... 85

9.1.1. Spectroscopic wavelength calibration techniques . . . . . . . . 85

9.1.2. Why use a captive gas as a wavelength reference? . . . . . . . . 89

9.2. Scope of this work . . . . . . . . . . . . . . . . . . . 91

10. Conventional absorption cell design 93

10.1. Design considerations . . . . . . . . . . . . . . . . . . 94

10.2. Short-path cell milestones . . . . . . . . . . . . . . . . . . . . 97

10.2.1. At optical wavelengths . . . . . . . . . . . . . . . . . 9 97

10.2.2. At near-infrared wavelengths . . . . . . . . . . . . . . 98

10.3. Shortcomings . . . . . . . . . . . . . . . . . . . . 101

10.3.1. Why White- or Herriott cells do not work . . . . . . . . . . . . 102

11. Novel absorption cell design $\quad 105$

11.1. Design principles ........................ . . 105

11.2. CRIRES requirements . . . . . . . . . . . . . . . 108

11.2.1. CRIRES key properties . . . . . . . . . . . . . . 108

11.2.2. Very Large Telescope key properties . . . . . . . . . . . . . . . 111

11.2.3. Restrictions imposed by the VLT . . . . . . . . . . . . . . . . . . . . 111

11.2.4. Restrictions imposed by the CRIRES instrument and its pre-optics . 114

11.2.5. VLT model . . . . . . . . . . . . . . . . . . . 116

11.3. A long-path cell concept for CRIRES . . . . . . . . . . . . . . . . 118

11.3.1. First order concept . . . . . . . . . . . . . . . . . 122

11.3.2. CRIRES model . . . . . . . . . . . . . . . . . . 123 
11.3.3. NAGICS system overview . . . . . . . . . . . . . . . . 128

11.3.4. Optical design ..................... . . . 129

11.3.5. Gas vessel design . . . . . . . . . . . . . . . . . . 134

12. Performance Analysis 139

12.1. Identifying a baseline design . . . . . . . . . . . . . . . . . 140

12.1.1. Opto-mechanical assembly height . . . . . . . . . . . . . . . . . . . 140

12.1.2. Optical bench dimensions . . . . . . . . . . . . . . . . . . . 141

12.1.3. Field of view . . . . . . . . . . . . . . . . . . . 142

12.1.4. Optical path length . . . . . . . . . . . . . . . . . 142

12.1.5. Mirror properties . . . . . . . . . . . . . . . . . . . . 144

12.1.6. Baseline design parameters . . . . . . . . . . . . . . . . . . . . . . . . . . . . . . . . . . 145

12.2. Model performance verification . . . . . . . . . . . . . . . . . . 145

12.2.1. VLT model at Nasmyth . . . . . . . . . . . . . . . . . . . 145

12.2.2. CRIRES model . . . . . . . . . . . . . . . . . . . . 146

12.3. Baseline design performance analysis . . . . . . . . . . . . . . . 156

12.3.1. Optical performance . . . . . . . . . . . . . . . . 157

12.3.2. Spectroscopic performance . . . . . . . . . . . . . . 167

12.3.3. Performance summary . . . . . . . . . . . . . . . . 173

13. Risk assessment 175

13.1. Trade-off options and simplifications . . . . . . . . . . . . . 175

13.1.1. Field of view . . . . . . . . . . . . . . . . . . . . . 175

13.1.2. Reducing optical complexities . . . . . . . . . . . . . . . . 176

13.1.3. Wavelength range . . . . . . . . . . . . . . . 178

13.2. Tolerances . . . . . . . . . . . . . . . . . . . 181

13.2.1. Optomechanical alignment . . . . . . . . . . . . . . . 182

13.2.2. Tolerance analysis . . . . . . . . . . . . . . . . 185

$\begin{array}{ll}\text { 14. Summary and outlook } & 187\end{array}$

14.1. Adaptions to other instruments . . . . . . . . . . . . . . . . . . . . . . 187

14.2. Summary .......................... . . 188

$\begin{array}{lr}\text { References } & 189\end{array}$

$\begin{array}{lr}\text { List of publications } & 205\end{array}$ 


\section{List of Figures}

3.1. Proper motion distribution in the Hyades . . . . . . . . . . . . . . . . 10

3.2. Distribution of candidate targets around the Hyades cluster center . . . . . 10

3.3. Colour magnitude diagrams of objects around the Hyades centre . . . . . . 11

3.4. Colour-colour diagrams of target candidates and cluster members . . . . . . 12

3.5. Distribution of spectral types of Hyades K-M stars covered . . . . . . . . . 12

4.1. Comparison of colour-temperature calibrations . . . . . . . . . . . 23

4.2. Comparison of effective temperatures obtained for the sample stars . . . . . 24

4.3. Colour vs. absolute magnitude diagram . . . . . . . . . . . . . . . . 31

4.4. $\mathrm{H} \alpha$ profiles of confirmed binaries . . . . . . . . . . . . . . . 32

5.1. $\mathrm{H} \alpha$ profiles of newly discovered (candidate) binaries . . . . . . . . . 40

5.2. Fractions of binary stars . . . . . . . . . . . . . . . . 41

5.3. Normalized $\mathrm{H} \alpha$ activity vs. effective temperature . . . . . . . . . . . . . 42

5.4. Normalized X-ray emission luminosity vs. effective temperature . . . . . . . 44

5.5. Chromospheric activity versus coronal activity . . . . . . . . . . . 45

5.6. Fraction of chromospherically active stars per spectral type . . . . . . . 47

5.7. Fraction of coronally active stars per spectral type . . . . . . . . . . . 48

5.8. Comparison of detected activity strengths with previous measurements . . . 49

5.9. Hyades measured rotational velocities $v_{\text {rot }} \sin i$ versus effective temperature $\quad 50$

5.10. Spectral type dependent ratios of rotating Hyades stars . . . . . . . . . 52

5.11. Distribution of projected rotational velocities $v \sin i$ with spectral type . . . $\quad 53$

5.12. Comparison of measured $v \sin i \ldots \ldots \ldots \ldots$. . . . . . . . . 54

5.13. Hyades chromospheric activity versus rotational velocity . . . . . . . . 55

5.14. Hyades coronal activity versus rotational velocity . . . . . . . . . . . 56

5.15. $\mathrm{H} \alpha$ line profiles for chromospherically inactive yet rotating stars . . . . . . 58

6.1. Fraction of Hyades stars with detected rotation . . . . . . . . . . . . 62

6.2. Comparison of chromospherically active Hyades and field stars . . . . . . 63

6.3. Comparison of spectroscopic and photometric rotational periods in the Hyades and Praesepe clusters . . . . . . . . . . . . . . . 66 66

9.1. Spectral line distribution of wavelength calibration sources . . . . . . . . 86

9.2. ThAr emission line strengths in the NIR . . . . . . . . . . . 87

9.3. $\mathrm{N}_{2} \mathrm{O}$ absorption line bands in the NIR . . . . . . . . . . . . 89

10.1. Stellar observations with an $\mathrm{HF}$ and $\mathrm{I}_{2}$ calibration gas cell . . . . . . . . . 97

10.2. CRIREs ammonia gascell vessel . . . . . . . . . . . . . . . . . . . . . 99

11.1. Conventional long-path gas cell collimation line concept . . . . . . . . . . 107

11.2. The Cryogenic High-Resolution Infrared Echelle Spectrograph (CRIRES) at the $\mathrm{VLT} \ldots \ldots \ldots \ldots$. . . . . . . . . . . . . . . . . . . . . . . . . . 
11.3. CRIREs Optical design . . . . . . . . . . . . . . . . . . . . . 110

11.4. Schematic drawing of the Vut unit telescope and dome . . . . . . . . . . 112

11.5. Arrangement of the CRIRES spectrograph on the Nasmyth platform (detail) 113

11.6. CRIRES optical layout (detail) . . . . . . . . . . . . . . . . . . 114

11.7. Optical model of the VLT in Nasmyth setup . . . . . . . . . . . . . . . . 117

11.8. 3D rendering of the optical VLT model . . . . . . . . . . . . . . . . 118

11.9. Footprint diagrams of selected field points along the VLT optical path . . . 119

11.10. Arrangement of CRIRES on the Nasmyth platform . . . . . . . . . . . . 120

11.11. Illustration of the collimation problem . . . . . . . . . . . . . . 121

11.12. Optical layout of the Vut Nasmyth focus . . . . . . . . . . . . . . . 123

11.13. Solution for a collimator with optimal collimation . . . . . . . . . . . . . 124

11.14. Solution for a collimator with the back-focal distance as a fixed parameter . 124

11.15. Solution for a collimator with the back-focal distance as an additional free parameter . . . . . . . . . . . . . . . . . . 124

11.16. CRIRES AO-relay model and derotator (topview) . . . . . . . . . . 126

11.17. Optical layout of the dichroic entrance window to the CRIRES vessel . . . . 128

11.18. NAGICS optical layout 3D system overview (bird's view) . . . . . . . . . . . 129

11.19. NAGics optical layout $2 \mathrm{D}$ (topview) . . . . . . . . . . . . . 130

11.20. FO sub-system optical layout (side view) . . . . . . . . . . . . . . . 133

11.21. DL sub-system optical layout (top view) . . . . . . . . . . . . . . . . 134

11.22. RCRF sub-system optical layout (top view) . . . . . . . . . . . . 135

11.23. 3D visualizations of the gas container vessel concept (side) . . . . . . . . . 137

11.24. 3D visualization of the gas container vessel concept (exploded view) . . . . 138

12.1. NAGics height parameter dependence . . . . . . . . . . . . . . . . 141

12.2. NAGICs folding mirror apertures versus field of view . . . . . . . . . . . 143

12.3. Mirror diameter as a function of field of view and cell length . . . . . . . . . 143

12.4. VLT model performance verification: spot size vs. field angle . . . . . . . . . 146

12.5. Vut model verification enclosed energy . . . . . . . . . . . . . . . . 147

12.6. Strehl ratios at the Nasmyth focal plane . . . . . . . . . . . . . . . . . . 148

12.7. Strehl ratios at the adaptive optics focal plane . . . . . . . . . . . . . 149

12.8. Strehl ratios at the instrument focal plane . . . . . . . . . . . . . . 150

12.9. Lateral color shift at the slit . . . . . . . . . . . . . . . . 151

12.10. Lateral color shift at the slit . . . . . . . . . . . . . . . . . 151

12.11. Strehl ratios as a function of defocus . . . . . . . . . . . . . . . 152

12.12. Encircled energy fractions at the slit $\left(50^{\prime \prime} \times 50^{\prime \prime}\right) \ldots \ldots \ldots \ldots$

12.13. Spot diagrams at the slit for $0.95,2.3$, and $5.3 \mu \mathrm{m}$ across $10^{\prime \prime} \times 10^{\prime \prime} \ldots \ldots 155$

12.14. Pupil image match on the AO-DM . . . . . . . . . . . . . . . 155

12.15. Measured reflectivity of HR metal coatings . . . . . . . . . . . . . 157

12.16. Integrated system transmission of the VLT and the CRIRES warm-optics (without gas cell) . . . . . . . . . . . . . . . . . . 157

12.17. Vignetting towards the field edges . . . . . . . . . . . . . . 159

12.18. Integrated system transmission of NAGICs for different coatings . . . . . . . 160

12.19. Image scale in the slit FP with and without NAGICS . . . . . . . . . . . . 161

12.20. Strehl ratio maps in the FP . . . . . . . . . . . . . . . 163

12.21. Strehl ratio vs. field angle in $\mathrm{X}$ and $\mathrm{Y} \ldots \ldots \ldots \ldots$. . . . . . . . 164

12.22. Wavelength dependent axial focal shift . . . . . . . . . . . . . . 164

12.23. Lateral color shift at the slit . . . . . . . . . . . . . . . . 165 
12.24. NAGICS encircled energy fractions at the slit . . . . . . . . . . . 166

12.25. Ratio of encircled energy fractions at the slit with and without NAGICS . . 167

12.26. Wavelength dependent RMS spot sizes across $10^{\prime \prime} \times 10^{\prime \prime}$ for NAGiCS and no cell168

12.27. Spot diagrams at the slit for $0.95,2.3$, and $5.3 \mu \mathrm{m}$ across $10^{\prime \prime} \times 10^{\prime \prime} \ldots \ldots$

12.28. Pupil image match on the AO-DM . . . . . . . . . . . . . . . . 170

12.29. NAGICS estimated performance with ammonia gas . . . . . . . . . . 171

12.30. NAGICS performance prediction for the methane-13 isotopologue . . . . . . 172

13.1. RMS spot sizes in the FP for the parabolic trade-off . . . . . . . . . . . 178

13.2. Encircled energy fractions at the slit for the parabolic trade-off option . . . 179

13.3. RMS spot radii in the FP for the spherical trade-off . . . . . . . . . . . . 180

13.4. Encircled energy fractions at the slit for the spherical trade-off option . . . 181

13.5. Resulting system transmission for alternative window substrates . . . . . . 182

13.6. System sensitivity to conic variation . . . . . . . . . . . . . 183

13.7. RSS estimates on performance changes . . . . . . . . . . . . . . . . . 184

13.8. Monte Carlo simulation results . . . . . . . . . . . . . . . . . . . 186 


\section{List of Tables}

3.1. Observed objects with identifiers, proper motions, optical and NIR magnitudes, and distances . . . . . . . . . . . . . . . . . 14

3.2. $\quad$ Log of FEROS and UVES observations . . . . . . . . . . . . . . 17

4.1. colour-temperature transformations . . . . . . . . . . . . 23

4.2. Spectral type and effective temperature . . . . . . . . . . . 25

4.3. Template stars used for the cross-correlation analysis . . . . . . . . . . 26

5.1. Confirmed and newly identified binaries . . . . . . . . . . . . . . . 37

5.2. Potential photometric binaries . . . . . . . . . . . . . . . . . 39

5.3. Activity and rotation results for $\mathrm{H} \alpha$ inactive yet rotating stars . . . . . . . 59

5.4. Comparison to other published rotational velocities $v \sin i \ldots \ldots$. . . . 60

A.1. Activity and rotation results for the Hyades combined catalog . . . . . . . 73

11.1. Vut optical design data for the Nasmyth configuration . . . . . . . . . 116

11.2. Crires-AO optical design data . . . . . . . . . . . . . . . 125

11.3. CRIRES pre-slit optical design data . . . . . . . . . . . . . . . 127

12.1. Summary of NAGiCs baseline design parameters . . . . . . . . . . . . 145

13.1. NAGICs trade-off for paraboli or spherical mirrors . . . . . . . . . . 176 


\section{List of Acronyms}

\begin{tabular}{ll} 
2MASS & Two Micron All-Sky Survey \\
ADC & Athmospheric dispersion compensator \\
AO & Adaptive optics \\
AODM & Adaptive optics deformable mirror \\
AOM & AO-mirror \\
AR & Anti-reflection \\
AU & Astronomical unit \\
BB & Blackbody \\
BCAH & Baraffe-Chabrier-Allard-Hauschildt stellar evolution models \\
CAT & Coud'e Auxiliary Telescope \\
CCD & Charged-coupled device \\
CCW & Counter-clockwise \\
CDU & Cross-disperser unit \\
CFHT & Canada-France-Hawaii Telescope \\
CMD & Color-magnitude diagram \\
CRIRES & Cryogenic High-Resolution Infrared Echelle Spectrograph \\
CSHELL & Cryogenic Echelle Spectrograph \\
DEC & Declination \\
DL & Diffraction limit \\
DM & deformable mirror \\
ESO & European Southern Observatory \\
EW & Equivalent width \\
FEROS & Fiber-fed Extended Range Optical Spectrograph \\
\hline FM & Folding miror \\
\hline
\end{tabular}


FOV Field of view

FP Focal plane

FTS Fourier-Transform spectrograph

FWHM Full-width at half maximum

HAIR $\quad \mathrm{H} \alpha$-inactive rotators

HARPS High-Accuracy Radial velocity Planet Searcher

HCL Hollow-cathode lamp

HITRAN High-resolution transmission molecular absorption database

HR high-resolution

HST Hubble Space Telescope

IF Interface

IMF Intermediate focus

INS Instrument

IR Infra-red

IRT Infra-red triplet

IRTF NASA Infra-red telescope facility

LFC Laser frequency comb

MACAO Multi Application Curvature Adaptive Optics

MAIT Manufacture, Assembly, Integration, Testing

MIDAS ESO-MIDAS data reduction system

MPG Max-Planck Gesellschaft

MS Main sequence

NAGICS Novel absorption gas cell design for the CRIRES spectrograph

NASA National Aeronautics and Space Administration

NIR Near infra-red

NIST Nastional Institute for Standards and Technology

NOMAD The Naval Observatory Merged Astrometric Dataset

OPD Optical path difference

OPL Optical path length 


\begin{tabular}{|c|c|}
\hline OPX & Optical axis \\
\hline PCEB & Post-common envelope binary \\
\hline $\mathrm{PM}$ & Proper motion \\
\hline PMS & Pre-main sequence \\
\hline PSF & Point-spread function \\
\hline PTV & Peak-to-valley \\
\hline RA & Right ascension \\
\hline RASS & ROSAT all-sky survey \\
\hline $\mathrm{RCM}$ & Re-collimating mirro \\
\hline $\mathrm{RCRF}$ & Re-collimation \& re-focusing \\
\hline RFM & Re-focusing mirror \\
\hline RMS & Root mean square \\
\hline ROC & Radius of curvature \\
\hline ROSAT & Röntgensatellit \\
\hline RSS & Root sum squared \\
\hline RV & Radial velocity \\
\hline SB & Spectroscopic binary \\
\hline SED & Spectral energy distribution \\
\hline SNR & Signal-to-noise ratio \\
\hline SPT & Spectral type \\
\hline TEL & Telescope \\
\hline TMA & Three mirror anasthigmat \\
\hline TNG & Telescopio Nazionale Galileo \\
\hline UCAC & The US Naval Observatory CCD Astrograph Catalog \\
\hline UKIRT & United Kingdom Infrared Telescope \\
\hline $\mathrm{UT}$ & Universal Time \\
\hline UV & Ultra-violet \\
\hline UVES & UV-Visible Echelle Spectrograph \\
\hline VLT & Very Large Telescope \\
\hline
\end{tabular}


WD

White dwarf

WFS

Wavefront sensor

$\mathrm{XCF}$

Cross-correlation function 



\section{Abstract}

High-resolution spectroscopy is employed to investigate a large sample of open cluster lowmass stars. The stellar rotation and magnetic activity of these stars are measured and their correlation is studied. I find in this sample that the same relation between these parameters holds on either side around the mass where stellar structure changes from partially to fully convective. These results promote a rotation driven magnetic dynamo mechanism in this whole mass regime for stars of intermediate ages $(\sim 625 \mathrm{Myr})$, and advocate against a correlation between rapid rotation prevalence and the transition to full convection.

In this work, also a new wavelength calibration device is developed for the technique of highresolution spectroscopy. This device enables unprecedented long pathlengths in transmission spectroscopy of absorption gases for wavelength referencing. The concept and design are presented and a detailed implementation of this design for a high-resolution near-infrared spectrograph (VLT/CRIRES) is modelled and characterized. The novel approach allows for higher precision wavelength calibration than existing techniques of similar kind. 



\section{Preface}

This thesis is presented in two parts, reflecting projects concerning high-resolution spectroscopy of low-mass stars. Part I presents a high-resolution spectroscopic study of low-mass stars in the Hyades open cluster. It investigates the connection between stellar rotation and stellar magnetic activity at the intermediate age of the Hyades, thereby probing observationally the magnetic field production in fully- and partially-convective main-sequence Hyades. The to date largest comprehensive catalog of this kind is presented. Early results have been published in Seemann et al. (2011); this study has been submitted for publication in Astronomy 8 Astrophysics.

Part II presents a novel concept of a wavelength calibration method for high-resolution optical and near-infrared spectroscopy. Such wavelength reference is in high demand for the spectroscopic detection and characterization of extrasolar planets, particularly of low-mass exoplanets around low-mass stars. For this purpose, the method presented here enables for the first time the application of gaseous transmission spectroscopy through long optical paths (several meters) as a simultaneous wavelength reference. An instrument baseline design of this novel concept is developed for the VLT/CRIRES spectrograph, and its performance analyzed. The concept is conceived in the context of the CRIRES + instrument upgrade; parts have been laid out in the technical report VLT-TRE-ESO-14500-5813 (Follert et al. 2013).

An abstract, introduction, and summary is provided for each part in this thesis. 



\section{Part I.}

Activity and Rotation in the Hyades 



\section{Abstract}

We present a catalog of rotation, chromospheric, and coronal activity in a sample of 171 low-mass stars in the Hyades open cluster. We explore the mass region where stars have not yet converged to slow rotation, but where instead rapid rotation becomes prevalent. The sample comprises spectral types from early-K to mid-M and covers the parameter space on both sides of the boundary to full convection. We obtain optical high-resolution spectra for 66 targets using ESO's FEROS and VLT/UVES facilities, and combine the data with measurements of $v \sin i, \mathrm{H} \alpha$ emission, and X-ray emission from the literature. We derive projected rotational velocities and chromospheric as well as coronal activity strengths, and infer homogenous effective temperatures and spectral types for all stars. Among the catalog stars, we identify 44 binaries or likely binaries. Five new highly probable low-mass binaries are discovered in the new spectroscopic data, as well as evidence for nine new photometric binary stars.

We find a steep rise in the fractions of chromospherically and of coronally active stars around spectral type K7/M0, with stars at earlier K-types being inactive, and with all stars showing activity by type M6, in agreement with previous findings. We discover a similarly steep increase in the fraction of rapid rotators at the same spectral types, based on our measurements of projected rotational velocities. On both sides of the boundary to full convection, we find a working rotation-activity connection in the $625 \mathrm{Myr}$ Hyades, so that rotation is always paralleled by activity. This indicates a rotation driven dynamo mechanism for solar-like and early to mid-M type Hyades alike, analogous to older stars.

A comparison of our results with data from the field supports the view that mass dependent magnetic braking is a function of stellar age, so that the threshold to rapid rotation occurs at higher masses in the intermediate age Hyades than in old field stars. In particular, our findings indicate that rapid rotation is not correlated to the boundary to full convection at $0.35 M_{\odot}$. Instead, we observe significant fractions of rapidly rotating low-mass stars at masses above $0.35 M_{\odot}$ in the Hyades, in contrast to field stars. We conclude that changes in the magnetic dynamo processes between partially and fully convective stars cannot be the underlying driver for the observed break in the rotation-distribution.

The distribution of projected rotation rates we find is further backed by photometric rotation periods. We discover a population of $\mathrm{H} \alpha$ inactive stars, yet with increased rotation rates that seem to violate the rotation-activity relation. We argue that these stars are nevertheless mildly active as evidenced in X-rays and Ca II emission, and that low to intermediate levels of activity are not mapped in $\mathrm{H} \alpha$ in these $\mathrm{K}$ and early M-type Hyades. 



\section{Introduction}

Solar-type stars are mostly fast rotators and magnetically active when they are young. Their magnetic fields, as evidenced from activity proxies (Güdel 2004; Schrijver \& Zwaan 2008), drive stellar winds (Roxburgh 1983) which rotationally slow-down the star by means of angular momentum transfer (Mestel 1984; Kawaler 1988). For solar-like stars $\left(0.35 \lesssim M_{\odot} \lesssim 1.3\right)$, the angular velocity evolution over time is empirically quantified by $\omega(t) \propto t^{-\frac{1}{2}}$ (Skumanich 1972). Spin-down times, ie. the period over which most of the angular momentum is dissipated (cf. MacGregor \& Brenner 1991), are thought to be strongly mass dependent (Barnes 2003). Following this rotation-age relation, higher mass stars like the sun are explained to have lost most of their angular velocity - gained during the contraction phase until the star reached the main-sequence (MS) - after a few hundred Myr.

On the contrary, lower-mass stars experience a longer pre-main-sequence (PMS) phase (Baraffe et al. 1998) and thus reach the MS at later ages. Once they settle on the MS, the smaller radii enforce spin-up velocities of order $100 \mathrm{~km} / \mathrm{s}$ (Bouvier et al. 1997). The brakingability is weaker than for higher masses, so that effectively, lower-mass stars on the MS are still spinning faster at any given age. As a result, the spin-down timescales of the latest solar-like stars (earlier than M3) are found of order 1 Gyr (eg. Barnes 2003; Irwin et al. 2011). This empirical framework is backed by a plethora of rotational periods, collected for solar-like stars in young open clusters and for field stars (eg. see the compilation in Irwin et al. 2011, and references therein).

The observational picture painted so far gets substantially more complex for very lowmass stars around and below the boundary to full convection: Though rotation data for M-stars has much increased in recent years, both in quality and numbers, the data mostly span only young stars of a few hundred Myr. At older ages, the determination of rotation rates is severely hampered by their intrinsic faintness, and rotation rates of field stars are sparse. As these stars settle on the MS at later ages than solar-like stars (a few hundred Myr compared to several $10 \mathrm{Myr}$ ), young clusters only probe very early stages of the Mdwarf braking history. A wide range of rotation rates is measured in young to intermediate cluster M-stars, and also in field M-dwarfs (eg. Marcy \& Chen 1992; Delfosse et al. 1998; Mohanty \& Basri 2003; Browning et al. 2010; Irwin et al. 2011; Reiners et al. 2012). The presence of slow and rapid rotators alike is ascribed to, first, reflect an imprint of the diversity during PMS spin-up evolution (spread in disc lifetimes); and second, to a much slower spindown process towards M-stars (Barnes 2003). Even at a Gyr age, the rotation rates of M-stars do not seem to have converged into a narrow band of velocities, whereas in the latest solar-like stars such a behaviour is observed before $600 \mathrm{Myr}$ (and thus resembles the braking evolution towards a uniform, slow rotation rate, at a given mass). Probing rotation in very late-type stars encounters additional difficulties, because the small stellar radii render the measurement of rotational velocities insensitive to slower rotators (eg. with a detection limit of $v \sin i \approx 3 \mathrm{~km} / \mathrm{s}$, the longest detectable rotation period is $P=3.3$ days, for a $0.2 R_{\odot}$ star). The excessive lengthening of the spin-down times in M-dwarfs is interpreted in terms of a strongly reduced magnetic braking efficiency, leaving them rapidly rotating $\left(v_{\text {rot }}>3 \mathrm{~km} / \mathrm{s}\right)$ even after Gyrs. A plausible explanation for the reduced braking efficiency is its strong 
dependence on stellar radius, which sharply drops at spectral type M3 (Reiners \& Mohanty 2012).

The turnover in braking efficiency between solar-type and very low-mass stars (approximately $0.35 M_{\odot}-0.08 M_{\odot}$ ) is encountered at around $0.35 M_{\odot}$, where stars become fully convective (Chabrier \& Baraffe 1997) and do not host a radiative core anymore. Models of magnetic dynamos (Ossendrijver 2003) predict that rotation driven dynamos (as observed in the sun) operate most efficiently at the bottom of the stellar convection zone. As the interface between convection zone and radiative core (tachocline) vanishes (Siess et al. 2000), a rotation-driven interface dynamo cannot operate anymore in fully convective stars (Chabrier \& Küker 2006). This has lead to the notion that the break in the rotation distribution diagnosed at around $0.3 M_{\odot}$ emerges from the changes in dynamo mechanism (Durney et al. 1993; Barnes 2003), thought to prevent effective rotational braking.

However, despite the changes in internal structure, M-stars are apparently still capable to produce strong magnetic fields, as evidenced by means of various activity tracers (Hawley et al. 1996; Gizis et al. 2002; West et al. 2004, 2008), by magnetic field measurements employing molecular Zeeman broadening (Reiners \& Basri 2007, 2010), and by Zeeman Doppler imaging (Donati et al. 2008; Morin et al. 2010).

A correspondence of activity and rotation, expressed as a well assessed rotation-activity relation in solar-like stars (Kraft 1970; Noyes et al. 1984; Pizzolato et al. 2003), therefore seems to also be at play in stars with masses below the boundary to full convection (Delfosse et al. 1998; Mohanty \& Basri 2003; Reiners \& Basri 2008; Reiners et al. 2012). It is understood as a continuation of the rotation-activity relation from higher mass stars, in that all active stars are also rapid rotators among the M-stars, and vice versa. Hence, rotation and activity are still linked by a dynamo at these lower masses with altered internal structure (Kiraga \& Stepien 2007; Wright et al. 2011). This generation of magnetic fields is believed to still reflect an underlying rotational dependence of the fully convective dynamo (Reiners \& Basri 2007; Reiners et al. 2012; Reiners \& Mohanty 2012), at least until the break-down of the rotation-activity relation around spectral type M7. For stars of type M7 and later types, rotation and activity do not seem to be correlated anymore (Reiners \& Basri 2010), although magnetic fields are still observed.

The transition (in terms of mass) from an inactive regime of solar-like (consequently, spundown) to the regime of very low-mass stars with pervasively observed high levels of activity (presumably fast rotators) is discernable in a few open clusters of intermediate ages (in the range 30 to $600 \mathrm{Myr}$ ) as an abrupt onset in $\mathrm{H} \alpha$ emission strength. This so-called $\mathrm{H} \alpha$-limit is evidenced at higher masses for the younger cluster ages (Hawley et al. 1999). Support to such a limit is drawn from the distribution of rotational periods in higher mass cluster stars, indicating a drop-off towards lower masses, but the threshold region between slow and rapid rotation in terms of mass (or spectral type) is sparsely sampled. It is thus elusive whether the activity limit at different ages also coincides with a shifted rotation limit - as would be suggested by a valid rotation-activity relation - or if the rotation threshold is keyed to the partial to convective boundary, as hinted at by the observational picture in older field stars.

The interpretation of the available data is somewhat clouded by a lack of cluster ages in the $300 \mathrm{Myr}-1$ Gyr parameter space range. On the younger end, the picture is diluted by the ongoing spin-up processes in M-stars. Conversely, on the older end among field stars, ages are hard to determine and mixed, and vary greatly (1-10 Gyr). Only a very limited number of open clusters with intermediate ages are available; at ages when their present lifetime is not long after the arrival on the MS, but yet below the spin-down timescales for M-type stars. Notably, there are the Hyades, Praesepe, and Coma at $\approx 600 \mathrm{Myr}$ which can serve to shed 
light on the corresponding stages of activity and rotation.

In the case of the Hyades open cluster, previous studies have reported declined rotation rates (Radick et al. 1987; Stauffer et al. 1987; Rebolo \& Beckman 1988; Delorme et al. 2011) for sun-like, mostly FG stars of the $625 \pm 50$ Myr population (Perryman et al. 1998). For mid M-type stars and later, high levels of rotation (Stauffer et al. 1997a; Reid \& Mahoney 2000) have been reported, alongside with chromospheric activity investigations (Stauffer et al. 1987; Hawley \& Reid 1994; Reid et al. 1995; Stauffer et al. 1997a; Hawley et al. 1999), but the rotational threshold region of early M-stars remains largely unexplored territory.

The present work adresses the gap of rotation and activity measurements in K-M-type stars, and aims to probe the link between chromospheric and coronal activity with respect to rotation at $625 \mathrm{Myr}$. The paper further aims to assess the view on rotation in early M-stars at an age where intermediate stages of the magnetic braking evolution in these stars can be tested. In particular, we probe whether the transition to full convection in the Hyades is correlated to a break in the distribution of rotation; ie. does the apparent linkage seen in field stars persist at younger ages?

In this first part of the present thesis, we present a catalog of rotation and activity in the Hyades K and M stars. It is organized as follows: Section 3 sets out to describe our strategy of sample selection and the new spectroscopic observations, followed by the treatment of the data. Our methodologies to measure activity strengths and rotational velocities using a crosscorrelation approach, as well as the determination of spectral types are detailed in Section 4. This section also discusses the choice of templates and our effort to identify multiple stars. We then present our results in Section 5, where we discuss binaries, and present strengths and rotation velocities. Their frequency and distributions are further looked at in the same section, where we also compare our results with previous ones, and elaborate on implications on the rotation-activity relation. The results are then discussed and put into context in Section 6, and summarized in Section 7. 



\section{Observations}

Contents

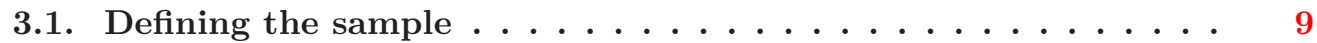
3.2. Observations ...................... 11
3.3. Data reduction . . . . . . . . . . . . . . . . 12

\subsection{Defining the sample}

To construct a representative sample of Hyades with spectral types $\mathrm{K}-\mathrm{M}$, we search for targets with constraints in proper motion, colour, and brightness. Candidates are drawn from the NOMAD database (Zacharias et al. 2004a) and additionally UCAC2 (Zacharias et al. 2004b) from within a $20^{\circ}$ radius around the Hyades cluster center. We disregard in our source catalog all objects that do not have known, high proper motions typical for Hyades members of $60 \lesssim \Delta \alpha \lesssim 150 \mathrm{mas} / \mathrm{yr}$ and $-60 \lesssim \Delta \delta \lesssim+10 \mathrm{mas} / \mathrm{yr}$ (Fig. 3.1). On the resulting $10^{5}$ stars, a constraint on $8 \lesssim V \lesssim 15$ and $6 \lesssim K \lesssim 10$ is applied (Fig. 3.2). From the 5500 remainders, we select only stars with $0.08 \lesssim H-K_{s} \lesssim 0.27,2.0 \lesssim V-K_{s} \lesssim 5.3$, and $2.3 \lesssim R-K_{s} \lesssim 5$, corresponding to spectral types between $\mathrm{K}$ and mid-M dwarfs (Tokunaga 2000). Fig. 3.3 and 3.4 illustrate the selection cuts. By selecting only stars within these colour ranges, we restrict ourselves to main sequence dwarf stars within spectral classes $\mathrm{K}$ and $\mathrm{M}$, and avoid giants as well as hotter main sequence stars. The remaining objects are crossmatched against the survey by Reid (1992). We do not apply a radial velocity criterion to constrain to the overall cluster motion, so to enhance the probability of cluster membership, as a selection based on radial velocities would limit us to objects with existing measurements of what we strive for. We exclude from the source catalog Hyades with genuine measurements of $\mathrm{H} \alpha$ and/or rotational velocities $v \sin i$ in Reid \& Mahoney (2000). Our final source catalog contains over 100 suitable candidates, with the targets distributed around the cluster core to ensure a good overlap with X-ray observations. A further restriction in declination $\left(\delta \lesssim 20^{\circ}\right)$ is performed for reasons of observability. All objects have $J H K$ magnitudes from 2MASS (Cutri et al. 2003), and optical magnitudes from various sources ( $B$ magnitudes are often unavailable or inconsistent, so we do not use those as constraints). Where $V R$ magnitudes are missing or inconsistent between the different catalogs used, $V R$ were compiled from the recent literature (eg. Ivanov (2008); Röser et al. (2011); Zacharias et al. (2010). Table 3.1 presents our source catalog and lists secondary object identifiers, proper motions, optical and NIR photometry, and distance information for all observed objects.

To expand the sample of low-mass Hyades of interest to us, we combine our catalog of observed targets with objects from the literature. We collect Hyades with previously measured $\mathrm{H} \alpha$ and $v \sin i$ and include objects from Radick et al. (1987); Stauffer et al. (1987, 1997a) and Reid \& Mahoney (2000). We consider only objects where previous works have determined both $\mathrm{H} \alpha$ and $v \sin i$, although many more data exists on $\mathrm{H} \alpha$ alone. From the totalling 171 Hyades we produce a combined catalog which we will call the combined sample in the 

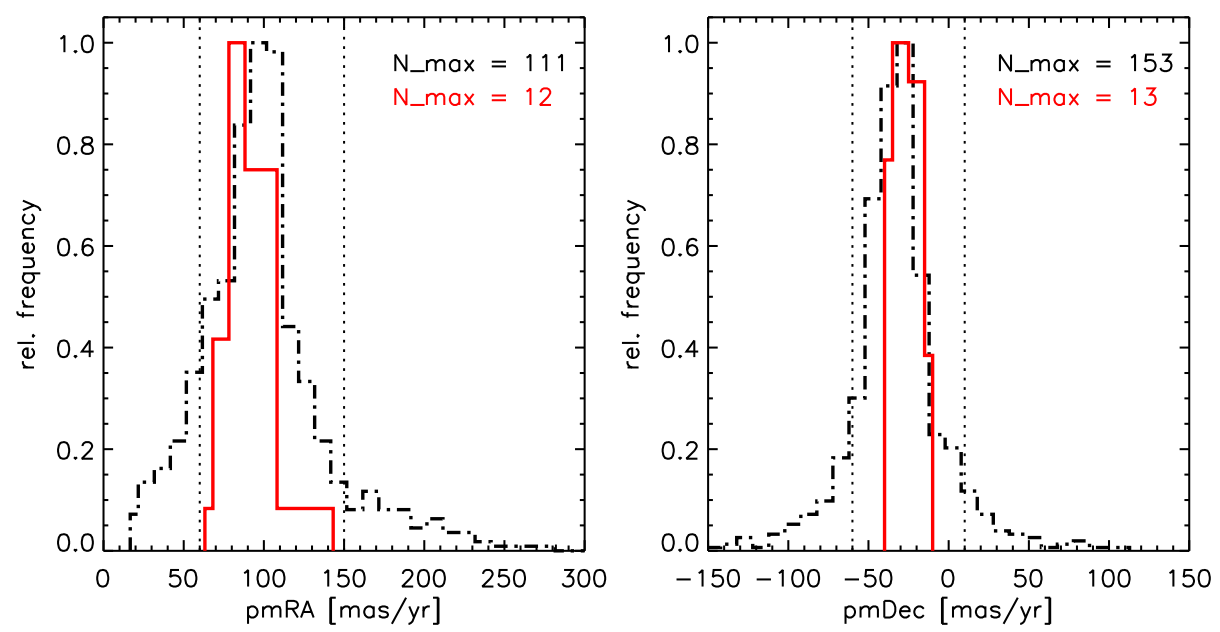

Figure 3.1.: Proper motion distribution in the Hyades (left panel in right ascension, right panel in declination). The dashed-dotted histogram shows cluster members from the catalog of Röser et al. (2011), which is not restricted to low-mass main sequence Hyades stars. Our source catalog selection (solid red line) is constructed around the proper motion center peaks in both RA and DEC, where the dotted lines represent the selection cuts. The relative frequency in each histogram is normalized by the number of objects in the central bin $N_{\max }$.
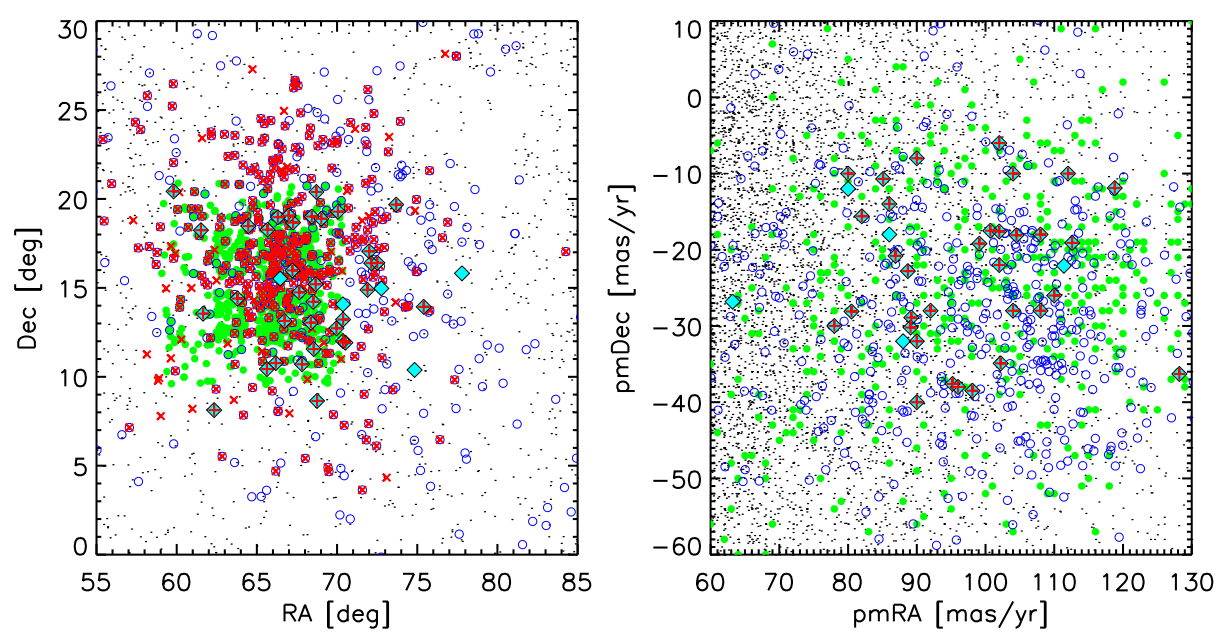

Figure 3.2.: left: Distribution of candidate targets around the Hyades cluster center $(\alpha=$ 04h $\left.26.9, \delta=+15^{\circ} 52^{\prime}\right)$. Plotted are stars from our input catalogs with constraints on proper motion and $V$ magnitude (dots), Hyades from the Reid (1992) catalog (green points), Hyades members from Röser et al. (2011, blue circles), and targets in our sample (cyan diamonds). Hyades with X-ray emission (Stern et al. 1995) are marked as red crosses (red plus signs for our targets). right: Distribution of proper motions for the same objects (with the same symbols). Note that the tight constraints on proper motion in RA and Dec places all targets in this work closely around the cluster center. 

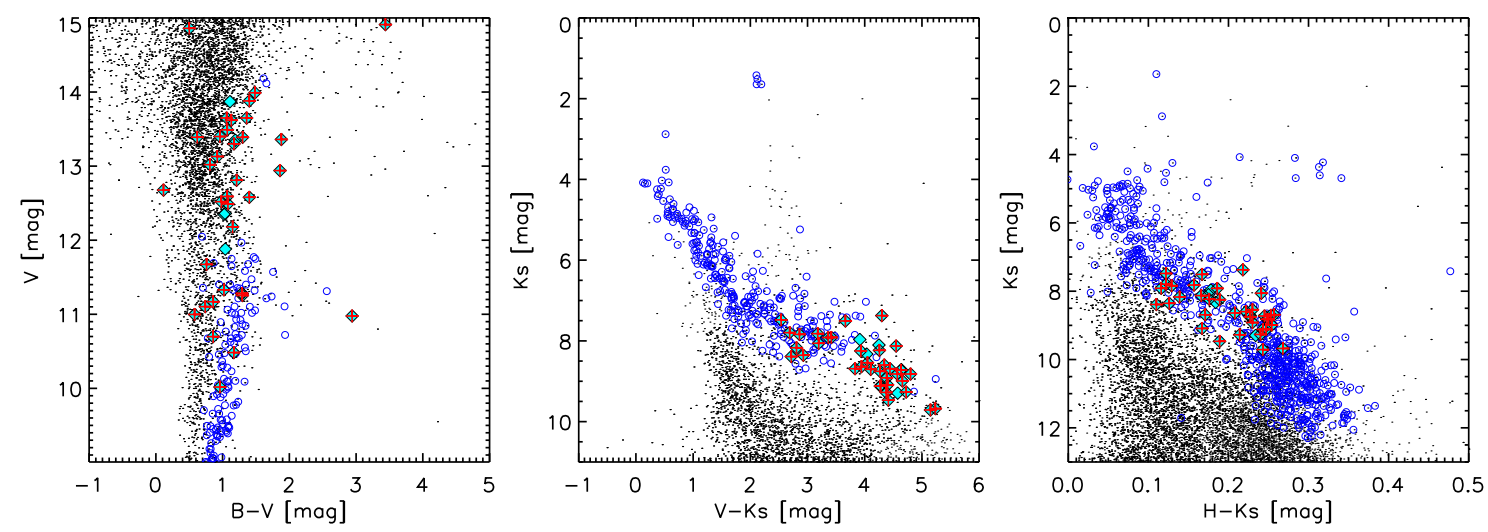

Figure 3.3.: Colour magnitude diagrams of objects around the Hyades centre. Black dots denote stars within $20^{\circ}$ from our combined input catalogs. All other symbols as in Fig. 3.2. Targets in our sample (diamonds) are required to lie within $0.08 \lesssim H-K_{s} \lesssim 0.27,2.0 \lesssim V-K_{s} \lesssim 5.3$. All selected objects occupy the Hyades main sequence between of spectral types $\mathrm{K}$ and M. Note the significantly smaller spread in the middle and right panels ( $B$ magnitudes seem to be less accurate than $V J H K)$.

following, with spectral types covering the range K1 to M6. Fig. 3.5 presents the distribution of spectral types covered.

\subsection{Observations}

Observations were performed for 66 of the stars in the source catalog. We obtained new spectroscopy for 55 objects listed in Table 3.2 using the Fiber-fed Extended Range Optical Spectrograph (FEROS), mounted on the ESO/MPG $2.2 \mathrm{~m}$ telescope on La Silla. FEROS (Kaufer \& Pasquini 1998) provides a wavelength coverage of $360 \mathrm{~nm}$ to $920 \mathrm{~nm}$ in 39 echelle orders and a fixed resolving power of $R=48000$. The secondary fiber was always placed on the nearby sky, serving as a sky background measurement. Integration times were typically 300-900 seconds for the K type stars, and 1200-3600 seconds for the M type objects. Three stars have more than one observation (cf. Table 3.2). We employed the atmospheric dispersion corrector (ADC) to compensate for losses at higher airmasses (typically $1.3-2.8$ ). A signalto-noise ratio $(\mathrm{S} / \mathrm{N})$ of up to 150 between $850-890 \mathrm{~nm}$ was achieved for the earlier type objects. For the M-dwarfs, where the SED falls off rapidly towards the blue, we obtain a S/N of typically around 90 , and $\gtrsim 60$ for the faintest targets in our sample $(V \lesssim 15)$.

Spectra for 11 additional objects observed with VLT/UVES (Dekker et al. 2000) were retrieved from the ESO archive facility. Observations were performed with resolving powers of $R=50000$ or 110000 (slit width $0.8^{\prime \prime}$ and $0.3^{\prime \prime}$ ), and in two different instrument modes covering wavelength ranges of $476-684 \mathrm{~nm}$ and $500-705 \mathrm{~nm}$ on two detectors in the red arm. We obtain a SNR of 40 for the latest type star (M3) and up to 350 for the early K stars in the red arm around $680 \mathrm{~nm}$ with exposure times between 300-1200 seconds, respectively. Table 3.2 summarizes the observations. 

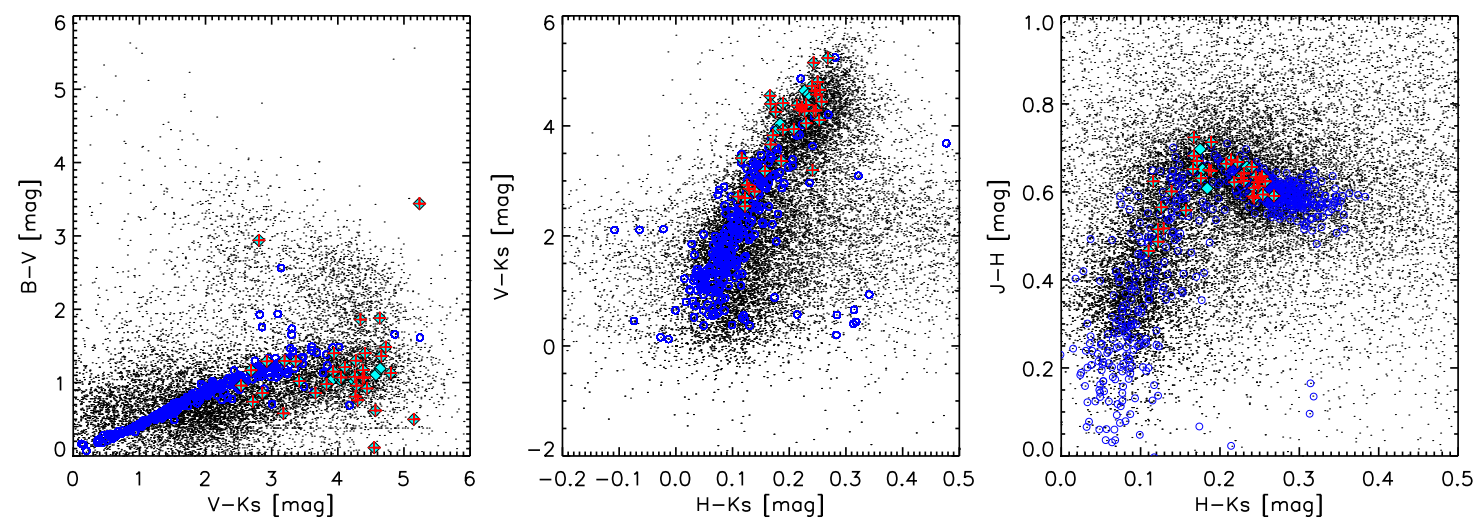

Figure 3.4.: Colour-colour diagrams of target candidates and cluster members, analogous to Fig. 3.3. Symbols as in Fig. 3.2. Our sample of Hyades is well constrained to the main sequence dwarf star region (Bessell \& Brett 1988). Optical colours seem to be rather unreliable for these late-type stars.

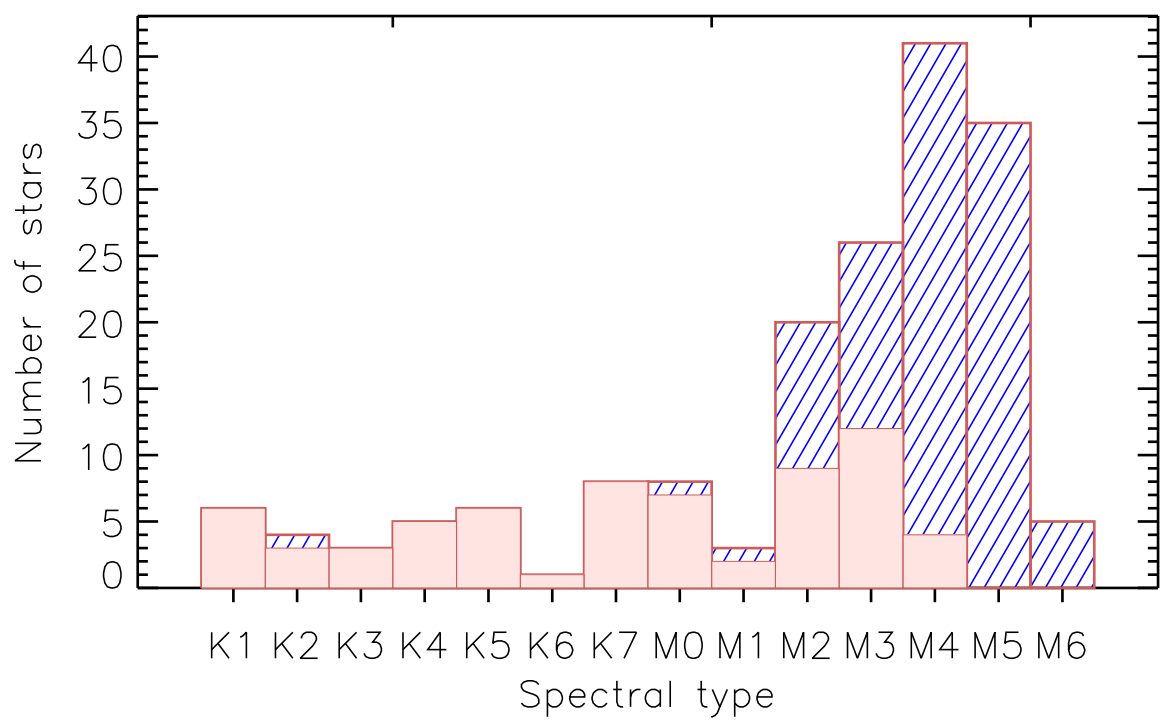

Figure 3.5.: Distribution of spectral types of Hyades K-M stars covered in this work. The total sample size is 171 (hatched histogram). Newly obtained spectroscopic observations are shown as solid histogram bars.

\subsection{Data reduction}

Calibration and reduction of the unbinned 2D echelle spectra was performed using the FEROS context within MIDAS. All raw spectra were bias subtracted and filtered for "cosmics", then order-traced, flat-fielded, optimal-extracted, and wavelength calibrated (using ThAr lamp exposures). A barycentric correction has been applied. A simultanously taken spectrum of the sky background (through the second fiber) was ultimately subtracted from each of the object spectra. For stars where multiple observations were taken, the individual spectra are averaged (RHy 57, RHy 299, LP 416-130). UVES raw data from the ESO archive were reduced with the corresponding full set of calibrations using the ESO UVES instrument pipeline 4.9.8. We treated the two red instrument arms independently to optimize the spectral fidelity. 
Optimal extraction was applied except for some of the highest SNR spectra, where optimal extraction would leave small scale ripples imprinted on the continuum flux level in the reddest orders. For the spectra affected, we could greatly reduce such residual artefacts by using an averaging extraction algorithm. Barycentric RV corrections were applied. Observations of rotational velocity template stars (FEROS and UVES) were treated following the same steps, respectively. 
Table 3.1.: Objects with identifiers, proper motions, optical and NIR magnitudes, and distances compiled for the observed sample stars. Sorted for spectral type. Object names refer to identifiers most commonly used in the literature.

\begin{tabular}{|c|c|c|c|c|c|c|c|c|c|c|c|c|}
\hline $\begin{array}{l}\text { Object } \\
\text { 2MASS J }\end{array}$ & other & $\begin{array}{c}\mu^{a} \\
\operatorname{mas} / \mathrm{yr}\end{array}$ & $\begin{array}{c}\epsilon^{a} \\
\operatorname{mas} / \mathrm{yr}\end{array}$ & $\begin{array}{c}\mathrm{B}^{b} \\
\mathrm{mag}\end{array}$ & $\begin{array}{c}\mathrm{V}^{b} \\
\mathrm{mag}\end{array}$ & $\begin{array}{c}\mathrm{R}^{c} \\
\mathrm{mag}\end{array}$ & $\begin{array}{c}\mathrm{J}^{d} \\
\mathrm{mag}\end{array}$ & $\begin{array}{c}\mathrm{H}^{d} \\
\mathrm{mag}\end{array}$ & $\begin{array}{l}\mathrm{K}_{S}{ }^{\mathrm{d}} \\
\mathrm{mag}\end{array}$ & $\mathrm{SpT}^{e}$ & $\begin{array}{c}\mathrm{d} \\
\mathrm{pc}\end{array}$ & Notes \\
\hline $03000280+0744590$ & $\mathrm{BD}+07459$ & 327.4 & 19.3 & 8.90 & 7.97 & 7.39 & 6.323 & 5.951 & 5.841 & K1 & 45.00 & c) \\
\hline $04092011+0807436$ & HG 7-113 & 85.1 & -10.7 & 11.84 & 11.10 & 10.62 & 8.964 & 8.498 & 8.388 & K1 & 66.36 & a) \\
\hline $03481186+0708464$ & LP 533-57 & 173.3 & 5.2 & 12.19 & 10.85 & 9.77 & 8.273 & 7.624 & 7.509 & K1 & 35.86 & a) \\
\hline $05004888+0443591$ & $\mathrm{BD}+04810$ & 73.4 & 10.7 & 10.65 & 9.72 & 9.16 & 8.113 & 7.692 & 7.589 & K1 & 49.60 & a) \\
\hline $04325009+1600210$ & vB 91; vA 684 & 101.7 & -28.5 & 9.83 & 8.90 & 8.33 & 7.285 & 6.859 & 6.770 & K1 & 45.98 & a) \\
\hline $04345007+2023404$ & LP 415-1399 & 98.1 & -38.5 & 12.35 & 11.33 & 10.70 & 8.654 & 8.030 & 7.914 & K1 & 47.08 & a) \\
\hline $04181926+1605181$ & vB 25 & 119.7 & -19.2 & 10.56 & 9.57 & 9.05 & 7.880 & 7.423 & 7.322 & $\mathrm{~K} 2$ & 44.11 & a) \\
\hline $04265434+1308175$ & LP $415-75$ & 104.5 & -18.1 & 11.65 & 10.48 & 9.77 & 8.432 & 7.917 & 7.795 & $\mathrm{~K} 2$ & 46.43 & a) \\
\hline $04395095+1243426$ & vB 311 & 102.0 & -17.6 & 10.98 & 10.02 & 9.42 & 8.089 & 7.602 & 7.480 & $\mathrm{~K} 2$ & 43.25 & a) \\
\hline $04082667+1211304$ & vB 233 & 116.0 & -13.0 & 12.60 & 11.27 & 10.61 & 8.818 & 8.171 & 8.046 & K3 & 47.80 & a) \\
\hline $04275674+1903390$ & HG 7-227 & 80.5 & -28.1 & 12.57 & 11.28 & 11.03 & 9.045 & 8.480 & 8.354 & K3 & 57.47 & a) \\
\hline $04500069+1624436$ & Pels 96 & 89.2 & -28.9 & 11.56 & 10.70 & 10.15 & 8.485 & 7.968 & 7.839 & K3 & 46.95 & a) \\
\hline $04033902+1927180$ & RHy 44 & 119.0 & -35.4 & 11.16 & 10.10 & 9.48 & 8.206 & 7.709 & 7.600 & K4 & 47.57 & a) \\
\hline $04322565+1306476$ & vB 288; vA 677 & 104.3 & -19.3 & 12.19 & 11.00 & 10.60 & 8.423 & 7.791 & 7.654 & K4 & 45.07 & a) \\
\hline $04473532+1453209$ & vB 332 & 88.7 & -22.8 & 10.71 & 10.98 & 11.04 & 8.910 & 8.309 & 8.170 & K4 & 47.04 & a) \\
\hline $04235440+1403075$ & vB 262; vA 294 & 114.2 & -15.6 & 12.16 & 11.02 & 10.80 & 8.506 & 7.925 & 7.786 & K4 & 43.27 & a) \\
\hline $04145191+1303178$ & vA 68 & 112.8 & -18.6 & 11.89 & 10.68 & 10.01 & 8.445 & 7.828 & 7.688 & $\mathrm{~K} 4$ & 47.06 & a) \\
\hline 04201057-1445400 & BD-15 767 & 176.8 & 113.3 & 10.98 & 9.78 & 9.21 & 7.554 & 6.976 & 6.832 & K5 & 28.64 & a) \\
\hline $03202921+0827161$ & G 79-28 & 226.3 & 3.2 & 10.77 & 9.63 & 8.91 & 7.545 & 6.955 & 6.881 & K5 & 31.09 & a) \\
\hline $04070122+1520062$ & Pels 15 & 126.0 & -20.7 & 11.67 & 10.47 & 9.40 & 8.365 & 7.811 & 7.665 & K5 & 44.50 & a) \\
\hline $04333716+2109030$ & vB 290 & 108.9 & -45.2 & 11.92 & 10.68 & 9.71 & 8.427 & 7.835 & 7.686 & K5 & 42.97 & a) \\
\hline $04094935+0918197$ & HG 7-115 & 155.8 & -2.8 & 11.29 & 10.10 & 9.44 & 7.912 & 7.340 & 7.264 & K5 & 35.35 & a) \\
\hline $04052565+1926316$ & vB 226 & 122.6 & -28.4 & 12.75 & 11.40 & 10.80 & 8.889 & 8.259 & 8.107 & K5 & 46.51 & a) \\
\hline $04334192+1900504$ & vB 291 & 102.2 & -34.9 & 11.58 & 11.00 & 10.61 & 8.535 & 7.977 & 7.820 & K6 & 45.35 & a) \\
\hline $04140812+0924240$ & HG 7-129 & 110.1 & -7.2 & 12.51 & 11.29 & 10.55 & 8.920 & 8.304 & 8.221 & $\mathrm{~K} 7$ & 45.00 & c) \\
\hline $04151038+1423544$ & vA 72 & 118.8 & -11.9 & 12.79 & 12.68 & 12.85 & 8.946 & 8.294 & 8.128 & K7 & 44.78 & a) \\
\hline $04063463+1332566$ & LP 474-1171 & 112.0 & -10.0 & 14.56 & 13.49 & 12.94 & 9.981 & 9.257 & 9.090 & K7 & 49.73 & a) \\
\hline
\end{tabular}


Table 3.1.: Observed objects (continued).

\begin{tabular}{|c|c|c|c|c|c|c|c|c|c|c|c|c|}
\hline $\begin{array}{l}\text { Object } \\
\text { 2MASS J }\end{array}$ & other & $\begin{array}{c}\mu^{a} \\
\operatorname{mas} / \mathrm{yr}\end{array}$ & $\begin{array}{r}\epsilon^{a} \\
\operatorname{mas} / \mathrm{yr}\end{array}$ & $\begin{array}{c}\mathrm{B}^{b} \\
\mathrm{mag}\end{array}$ & $\begin{array}{c}\mathrm{V}^{b} \\
\mathrm{mag}\end{array}$ & $\begin{array}{c}\mathrm{R}^{c} \\
\mathrm{mag}\end{array}$ & $\begin{array}{c}\mathrm{J}^{d} \\
\mathrm{mag}\end{array}$ & $\begin{array}{c}\mathrm{H}^{d} \\
\mathrm{mag}\end{array}$ & $\begin{array}{l}\mathrm{K}_{S}{ }^{d} \\
\mathrm{mag}\end{array}$ & $\mathrm{SpT}^{e}$ & $\begin{array}{c}\mathrm{d} \\
\mathrm{pc}\end{array}$ & Notes \\
\hline $04412966+1313164$ & vB 316 & 99.1 & -19.2 & 12.03 & 11.17 & 10.62 & 8.356 & 7.674 & 7.507 & $\overline{\mathrm{K} 7}$ & 45.00 & c) \\
\hline $04340530+1413029$ & vA 731 & 82.0 & -15.6 & 13.50 & 12.52 & 11.30 & 9.531 & 8.861 & 8.690 & K7 & 58.51 & a) \\
\hline $04081110+1652229$ & HG 7-104 & 133.2 & -23.0 & 13.29 & 11.12 & 10.41 & 8.766 & 8.106 & 7.933 & K7 & 42.09 & a) \\
\hline $05110971+1548574$ & $\ldots$ & 63.2 & -26.8 & 13.37 & 12.35 & 11.32 & 8.974 & 8.277 & 8.102 & K7 & 45.00 & c) \\
\hline $04343992+1512325$ & vA 750 & 104.0 & -28.0 & 13.57 & 12.49 & 11.40 & 9.041 & 8.410 & 8.234 & K7 & 45.29 & a) \\
\hline $04510241+1458167$ & $\ldots$ & 88.0 & -32.0 & 12.92 & 11.88 & 10.81 & 8.795 & 8.144 & 7.966 & M0 & 45.00 & c) \\
\hline $04161310+1853042$ & vB 247 & 119.6 & -33.6 & 13.30 & 11.86 & 11.12 & 9.062 & 8.448 & 8.268 & M0 & 44.29 & a) \\
\hline $04254922+1531165$ & vA 366 & 111.4 & -22.1 & 13.40 & 12.36 & 11.41 & 9.118 & 8.510 & 8.326 & M0 & 45.00 & c) \\
\hline $05013603+1355586$ & vB 348 & 86.9 & -20.8 & 12.57 & 11.28 & 10.48 & 8.754 & 8.104 & 7.918 & M0 & 42.25 & a) \\
\hline $04222568+1118205$ & vB 259 & 118.7 & -12.3 & 11.05 & 9.82 & 9.20 & 7.620 & 7.135 & 6.948 & M0 & 42.70 & a) \\
\hline $04285243+1558539$ & vA 502 & 112.6 & -19.1 & 13.33 & 12.18 & 11.30 & 9.151 & 8.438 & 8.249 & M0 & 46.30 & b) \\
\hline $04244401+1046192$ & LP 475-60 & 104.0 & -10.0 & 15.28 & 13.88 & 13.56 & 10.304 & 9.656 & 9.467 & M0 & 48.90 & a) \\
\hline $03565784+1650581$ & HG 7-46 & 83.2 & -60.7 & 18.24 & 12.02 & 11.17 & 9.465 & 8.825 & 8.622 & M1 & 45.00 & c) \\
\hline $04223004+1026046$ & LP $475-445$ & 102.0 & -6.0 & 13.98 & 12.58 & 12.10 & 9.510 & 8.846 & 8.638 & M1 & 49.00 & a) \\
\hline $04544410+1940513$ & LP 416-130 & 90.0 & -40.0 & 14.71 & 13.65 & 13.45 & 10.163 & 9.490 & 9.276 & M2 & 44.42 & a) \\
\hline $04480086+1703216$ & LP 416-43 & 89.1 & -30.2 & 12.43 & 11.67 & 11.18 & 8.214 & 7.592 & 7.374 & M2 & 48.08 & a) \\
\hline $04291234+1516259$ & vA 529 & 100.1 & -32.9 & 12.90 & 12.34 & 11.20 & 9.308 & 8.706 & 8.486 & M2 & 47.24 & a) \\
\hline $04060221+1815033$ & LP 414-54 & 149.9 & -34.1 & 14.80 & 12.94 & 12.30 & 9.488 & 8.820 & 8.598 & M2 & 39.75 & a) \\
\hline $04404249+0213522$ & $\ldots$ & 170.6 & 49.4 & 12.58 & 11.05 & 10.31 & 7.894 & 7.255 & 7.032 & M2 & 25.38 & a) \\
\hline $04412876+1200337$ & LP 475-1699 & 86.0 & -14.0 & 13.83 & 13.02 & 11.91 & 9.612 & 8.980 & 8.753 & M2 & 46.38 & a) \\
\hline $04412780+1404340$ & $\ldots$ & 86.0 & -18.0 & 14.54 & 13.35 & 12.35 & 9.576 & 8.938 & 8.711 & M2 & 40.87 & a) \\
\hline $04360416+1853189$ & LP 415-1582 & 90.0 & -32.0 & 14.47 & 13.30 & 13.53 & 9.772 & 9.147 & 8.917 & M2 & 51.20 & a) \\
\hline $04483062+1623187$ & LP 416-570 & 78.0 & -30.0 & 13.66 & 12.59 & 11.36 & 9.414 & 8.772 & 8.542 & M2 & 50.25 & a) \\
\hline $04591981+1023096$ & $\ldots$ & 80.0 & -12.0 & 14.98 & 13.87 & 12.69 & 10.185 & 9.528 & 9.294 & M3 & 50.25 & a) \\
\hline $04350255+0839304$ & LP 415-292 & 95.2 & -37.6 & 15.48 & 13.99 & 12.70 & 10.163 & 9.506 & 9.266 & M3 & 52.97 & a) \\
\hline $04271663+1714305$ & vA 420 & 109.0 & -35.0 & 13.89 & 13.28 & 12.40 & 9.714 & 9.067 & 8.826 & M3 & 44.46 & a) \\
\hline $04341113+1133285$ & vB 294 & 100.7 & -17.5 & 12.55 & 11.25 & 10.45 & 8.890 & 8.300 & 8.059 & M3 & 51.05 & a) \\
\hline $04303385+1444532$ & LP $475-957$ & 102.0 & -22.0 & 15.36 & 14.86 & 13.57 & 10.563 & 9.954 & 9.711 & M3 & 49.24 & a) \\
\hline
\end{tabular}


Table 3.1.: Observed objects (continued).

\begin{tabular}{|c|c|c|c|c|c|c|c|c|c|c|c|c|}
\hline $\begin{array}{l}\text { Object } \\
\text { 2MASS J }\end{array}$ & other & $\begin{array}{r}\mu^{a} \\
\mathrm{mas} / \mathrm{yr}\end{array}$ & $\begin{array}{r}\epsilon^{a} \\
\mathrm{mas} / \mathrm{yr}\end{array}$ & $\begin{array}{r}\mathrm{B}^{b} \\
\mathrm{mag}\end{array}$ & $\begin{array}{c}\mathrm{V}^{b} \\
\mathrm{mag}\end{array}$ & $\begin{array}{c}\mathrm{R}^{c} \\
\mathrm{mag}\end{array}$ & $\begin{array}{r}\mathrm{J}^{d} \\
\mathrm{mag}\end{array}$ & $\begin{array}{c}\mathrm{H}^{d} \\
\mathrm{mag}\end{array}$ & $\begin{array}{l}\mathrm{K}_{S}{ }^{d} \\
\mathrm{mag}\end{array}$ & $\mathrm{SpT}^{e}$ & $\begin{array}{c}\mathrm{d} \\
\mathrm{pc}\end{array}$ & Notes \\
\hline $04401271+1917099$ & vB 310 & 96.0 & -38.0 & 14.69 & 13.39 & 13.66 & 9.948 & 9.362 & 9.119 & M3 & 46.45 & a) \\
\hline $04223953+1816097$ & LP 415-27 & 128.2 & -36.3 & 15.24 & 13.36 & 12.20 & 9.605 & 8.971 & 8.726 & M3 & 36.75 & a) \\
\hline $04420453+1155137$ & LP $475-1747$ & 80.0 & -10.0 & 14.36 & 13.40 & 12.48 & 9.987 & 9.365 & 9.118 & M3 & 53.68 & a) \\
\hline $04322373+1745026$ & vA 673 & 107.9 & -38.3 & 17.83 & 13.89 & 14.14 & 10.753 & 10.161 & 9.913 & M3 & 43.16 & a) \\
\hline $04295572+1654506$ & vA 559 & 111.3 & -30.6 & 13.79 & 13.07 & 11.91 & 9.524 & 8.901 & 8.653 & M3 & 43.20 & a) \\
\hline $04332699+1302438$ & vA 709 & 108.0 & -18.0 & 14.01 & 13.39 & 12.13 & 9.682 & 9.069 & 8.821 & M3 & 41.84 & a) \\
\hline $03591350+2025415$ & LP 414-479 & 138.0 & -28.0 & 14.75 & 13.62 & 12.84 & 9.700 & 9.070 & 8.820 & M3 & 45.00 & c) \\
\hline $04251456+1858250$ & LP 415-794 & 92.0 & -28.0 & 14.02 & 12.81 & 12.95 & 9.593 & 8.958 & 8.705 & M4 & 51.49 & a) \\
\hline $04175061+1828307$ & vB 250 & 110.0 & -26.0 & 15.01 & 13.65 & 13.16 & 9.840 & 9.246 & 8.992 & M4 & 46.69 & a) \\
\hline $04290099+1840254$ & vB 278 & 108.0 & -28.0 & 14.05 & 13.13 & 12.96 & 9.570 & 8.946 & 8.689 & M4 & 46.21 & a) \\
\hline $04311576+1042154$ & RHy 299 & 90.0 & -8.0 & 16.28 & 14.91 & 13.66 & 10.535 & 9.944 & 9.676 & M4 & 44.84 & a) \\
\hline
\end{tabular}

References. Sources if not stated otherwise: ${ }^{(a)}$ RA, Dec (Table 3.2) and proper motions from NOMAD (Zacharias et al. 2004a), (b) BV from Röser et al. (2011), (c) R magnitude from NOMAD, (d) 2 MASS $\mathrm{JHK}_{S}$ from Cutri et al. (2003), (e) see section 4.1

Notes. Distances derived from parallaxes in a) Röser et al. (2011), b) Hanson (1975) or Schwan (1991); c) fixed to 45 pc (cluster center). 
Table 3.2.: Log of observations for the 66 objects observed with the FEROS and UVES spectrographs, sorted by spectral type.

\begin{tabular}{|c|c|c|c|c|c|c|c|c|c|}
\hline $\begin{array}{c}\text { Target }^{a} \\
\text { 2MASS J }\end{array}$ & Other Name ${ }^{b}$ & $\alpha(J 2000)$ & $\delta(J 2000)$ & $\begin{array}{c}\text { Date } \\
\text { MJD-2400000 }\end{array}$ & $\mathrm{SpT}$ & $\begin{array}{c}\mathrm{V} \\
\mathrm{mag}\end{array}$ & $\begin{array}{l}\text { Exptime } \\
\text { sec }\end{array}$ & $\begin{array}{l}\operatorname{Ins}^{c} \\
F / U\end{array}$ & $\mathrm{SNR}^{d}$ \\
\hline $03000280+0744590$ & $\mathrm{BD}+07459$ & 030002.8 & +074459.1 & 54398.09 & K1 & 7.97 & 900 & $\mathrm{~F}$ & 364 \\
\hline $04092011+0807436$ & HG 7-113 & 040920.1 & +080743.7 & 54783.37 & K1 & 11.10 & 600 & $\mathrm{~F}$ & 78 \\
\hline $03481186+0708464$ & LP 533-57 & 034811.9 & +070846.5 & 51919.07 & K1 & 10.85 & 1200 & $\mathrm{U}$ & 201 \\
\hline $05004888+0443591$ & $\mathrm{BD}+04810$ & 050048.9 & +044359.2 & 52649.16 & K1 & 9.72 & 900 & $\mathrm{U}$ & 184 \\
\hline $04325009+1600210$ & vB 91; vA 684 & 043250.1 & +160020.9 & 55126.25 & K1 & 8.90 & 1800 & $\mathrm{~F}$ & 69 \\
\hline $04345007+2023404$ & LP 415-1399 & 043450.1 & +202340.5 & 54782.24 & K1 & 11.33 & 600 & $\mathrm{~F}$ & 105 \\
\hline $04181926+1605181$ & vB 25 & 041819.3 & +160518.0 & 52657.05 & $\mathrm{~K} 2$ & 9.57 & 300 & U & 68 \\
\hline $04265434+1308175$ & LP $415-75$ & 042654.3 & +130817.4 & 54788.31 & $\mathrm{~K} 2$ & 10.48 & 300 & $\mathrm{~F}$ & 74 \\
\hline $04395095+1243426$ & vB 311 & 043951.0 & +124342.5 & 54786.34 & K2 & 10.02 & 300 & $\mathrm{~F}$ & 94 \\
\hline $04082667+1211304$ & vB 233 & 040826.7 & +121130.6 & 55768.41 & K3 & 11.27 & 480 & $\mathrm{~F}$ & 56 \\
\hline $04275674+1903390$ & HG 7-227 & 042756.8 & +190338.9 & 54782.26 & K3 & 11.28 & 600 & $\mathrm{~F}$ & 75 \\
\hline $04500069+1624436$ & Pels 96 & 045000.7 & +162443.4 & 54787.35 & K3 & 10.70 & 300 & $\mathrm{~F}$ & 69 \\
\hline $04033902+1927180$ & RHy 44 & 040339.0 & +1927 18.0 & 52656.09 & K4 & 10.10 & 1200 & $\mathrm{U}$ & 350 \\
\hline $04322565+1306476$ & vB 288; vA 677 & 043225.7 & +130647.6 & 55767.44 & K4 & 11.00 & 600 & $\mathrm{~F}$ & 41 \\
\hline $04473532+1453209$ & vB 332 & 044735.3 & +145320.7 & 54782.28 & K4 & 10.98 & 600 & $\mathrm{~F}$ & 81 \\
\hline $04235440+1403075$ & vB 262; vA 294 & 042354.4 & +140307.6 & 55772.46 & K4 & 11.02 & 332 & $\mathrm{~F}$ & 16 \\
\hline $04145191+1303178$ & vA 68 & 041451.9 & +130317.8 & 52561.27 & K4 & 10.68 & 398 & $\mathrm{U}$ & 75 \\
\hline 04201057-1445400 & BD-15 767 & 042010.6 & -144539.9 & 55198.14 & K5 & 9.78 & 900 & $\mathrm{~F}$ & 210 \\
\hline $03202921+0827161$ & G 79-28 & 032029.2 & +082716.0 & 52656.08 & K5 & 9.63 & 300 & $\mathrm{U}$ & 224 \\
\hline $04070122+1520062$ & Pels 15 & 040701.2 & +152006.1 & 53329.12 & K5 & 10.47 & 600 & $\mathrm{U}$ & 142 \\
\hline $04333716+2109030$ & vB 290 & 043337.2 & +210903.1 & 52561.31 & K5 & 10.68 & 399 & $\mathrm{U}$ & 96 \\
\hline $04094935+0918197$ & HG 7-115 & 040949.3 & +091819.8 & 52663.10 & K5 & 10.10 & 1200 & $\mathrm{U}$ & 296 \\
\hline $04052565+1926316$ & vB 226 & 040525.7 & +192631.8 & 55772.42 & K5 & 11.40 & 600 & $\mathrm{~F}$ & 36 \\
\hline $04334192+1900504$ & vB 291 & 043341.9 & +190050.5 & 54782.25 & K6 & 11.00 & 600 & $\mathrm{~F}$ & 102 \\
\hline $04140812+0924240$ & HG 7-129 & 041408.1 & +092424.1 & 55772.45 & K7 & 11.29 & 840 & $\mathrm{~F}$ & 49 \\
\hline $04151038+1423544$ & vA 72 & 041510.4 & +142354.5 & 54785.14 & K7 & 12.68 & 2100 & $\mathrm{~F}$ & 116 \\
\hline $04063463+1332566$ & LP $474-1171$ & 040634.6 & +133256.8 & 54785.11 & K7 & 13.49 & 2400 & $\mathrm{~F}$ & 57 \\
\hline
\end{tabular}


Table 3.2.: Log of observations for the 66 objects observed with the FEROS and UVES spectrographs, sorted by spectral type (continued).

\begin{tabular}{|c|c|c|c|c|c|c|c|c|c|}
\hline $\begin{array}{c}\text { Target }^{a} \\
\text { 2MASS J }\end{array}$ & Other Name $^{b}$ & $\alpha(J 2000)$ & $\overline{\delta(J 2000)}$ & $\begin{array}{c}\text { Date } \\
\text { MJD-2400000 }\end{array}$ & $\overline{\mathrm{SpT}}$ & $\begin{array}{l}\mathrm{V} \\
\mathrm{mag}\end{array}$ & $\begin{array}{l}\text { Exptime } \\
\text { sec }\end{array}$ & $\begin{array}{l}\operatorname{Ins}^{c} \\
\mathrm{~F} / \mathrm{U}\end{array}$ & $\overline{\mathrm{SNR}^{d}}$ \\
\hline $04412966+1313164$ & vB 316 & 044129.7 & +131316.3 & 54782.30 & K7 & 11.17 & 600 & $\mathrm{~F}$ & 101 \\
\hline $04340530+1413029$ & vA 731 & 043405.3 & +141303.2 & 54786.32 & K7 & 12.52 & 1000 & $\mathrm{~F}$ & 68 \\
\hline $04081110+1652229$ & HG 7-104 & 040811.1 & +165223.1 & 55772.43 & K7 & 11.12 & 700 & $\mathrm{~F}$ & 41 \\
\hline $05110971+1548574$ & $\ldots$ & 051109.7 & +154857.5 & 54781.29 & K7 & 12.35 & 1200 & $\mathrm{~F}$ & 104 \\
\hline $04343992+1512325$ & vA 750 & 043439.9 & +151232.7 & 54782.32 & K7 & 12.49 & 720 & $\mathrm{~F}$ & 76 \\
\hline $04510241+1458167$ & & 045102.4 & +1458 16.5 & 54782.29 & M0 & 11.88 & 600 & $\mathrm{~F}$ & 86 \\
\hline $04161310+1853042$ & vB 247 & 041613.1 & +185304.2 & 55772.44 & M0 & 11.86 & 840 & $\mathrm{~F}$ & 43 \\
\hline $04254922+1531165$ & vA 366 & 042549.2 & +153116.5 & 54786.28 & M0 & 12.36 & 1000 & $\mathrm{~F}$ & 74 \\
\hline $05013603+1355586$ & vB 348 & 050136.0 & +135558.8 & 54781.28 & M0 & 11.28 & 600 & $\mathrm{~F}$ & 83 \\
\hline $04222568+1118205$ & vB 259 & 042225.7 & +11 1820.6 & 55125.25 & M0 & 9.82 & 1800 & $\mathrm{~F}$ & 46 \\
\hline $04285243+1558539$ & vA 502 & 042852.5 & +155853.8 & 54788.30 & M0 & 12.18 & 1000 & $\mathrm{~F}$ & 101 \\
\hline $04244401+1046192$ & LP 475-60 & 042444.0 & +104619.6 & 54784.28 & M0 & 13.88 & 4200 & $\mathrm{~F}$ & 103 \\
\hline $03565784+1650581$ & HG 7-46 & 035657.9 & +165058.2 & 55769.43 & M1 & 12.02 & 780 & $\mathrm{~F}$ & 19 \\
\hline $04223004+1026046$ & LP $475-445$ & 042230.0 & +102604.8 & 54784.26 & M1 & 12.58 & 1400 & $\mathrm{~F}$ & 99 \\
\hline $04544410+1940513$ & LP 416-130 & 045444.1 & +194051.3 & 54787.27 & M2 & 13.65 & 2000 & $\mathrm{~F}$ & 89 \\
\hline $04480086+1703216$ & LP 416-43 & 044800.9 & +170321.7 & 54783.32 & M2 & 11.67 & 900 & $\mathrm{~F}$ & 153 \\
\hline $04291234+1516259$ & vA 529 & 042912.4 & +151626.0 & 52657.07 & M2 & 12.34 & 300 & $\mathrm{U}$ & 36 \\
\hline $04060221+1815033$ & LP 414-54 & 040602.6 & +18 1432.4 & 54786.18 & M2 & 12.94 & 1000 & $\mathrm{~F}$ & 69 \\
\hline $04404249+0213522$ & $\ldots$ & 044042.5 & +02 1352.0 & 53710.25 & M2 & 11.05 & 938 & $\mathrm{~F}$ & 144 \\
\hline $04412876+1200337$ & LP 475-1699 & 044128.8 & +120033.7 & 54784.23 & M2 & 13.02 & 1800 & $\mathrm{~F}$ & 107 \\
\hline $04412780+1404340$ & & 044127.8 & +140434.1 & 54783.30 & M2 & 13.35 & 1500 & $\mathrm{~F}$ & 97 \\
\hline $04360416+1853189$ & LP 415-1582 & 043604.2 & +185319.0 & 54788.25 & M2 & 13.30 & 3600 & $\mathrm{~F}$ & 121 \\
\hline $04483062+1623187$ & LP 416-570 & 044830.6 & +16 2319.0 & 54782.27 & M2 & 12.59 & 600 & $\mathrm{~F}$ & 57 \\
\hline $04591981+1023096$ & $\ldots$ & 045919.8 & +102309.5 & 54784.33 & M3 & 13.87 & 2100 & $\mathrm{~F}$ & 79 \\
\hline $04350255+0839304$ & LP 415-292 & 043500.6 & +083846.2 & 54781.31 & M3 & 13.99 & 900 & $\mathrm{~F}$ & 116 \\
\hline $04271663+1714305$ & vA 420 & 042716.6 & +17 1430.5 & 55770.44 & M3 & 13.28 & 2121 & $\mathrm{~F}$ & 32 \\
\hline $04341113+1133285$ & vB 294 & 043411.2 & +113328.5 & 54782.31 & M3 & 11.25 & 900 & $\mathrm{~F}$ & 93 \\
\hline $04303385+1444532$ & LP 475-957 & 043033.9 & +14 4453.2 & 54787.29 & M3 & 14.86 & 4200 & $\mathrm{~F}$ & 81 \\
\hline
\end{tabular}


Table 3.2.: Log of observations for the 66 objects observed with the FEROS and UVES spectrographs, sorted by spectral type (continued).

\begin{tabular}{|c|c|c|c|c|c|c|c|c|c|}
\hline $\begin{array}{c}\text { Target }^{a} \\
\text { 2MASS J }\end{array}$ & Other Name ${ }^{b}$ & $\alpha(J 2000)$ & $\delta(J 2000)$ & $\begin{array}{c}\text { Date } \\
\text { MJD-2400000 }\end{array}$ & $\overline{\mathrm{SpT}}$ & $\begin{array}{c}\mathrm{V} \\
\mathrm{mag}\end{array}$ & $\begin{array}{l}\text { Exptime } \\
\text { sec }\end{array}$ & $\begin{array}{l}\mathrm{Ins}^{c} \\
\mathrm{~F} / \mathrm{U}\end{array}$ & $\overline{\text { SNR }}^{d}$ \\
\hline $04401271+1917099$ & vB 310 & 044012.7 & +191710.0 & 54788.21 & M3 & 13.39 & 3599 & $\mathrm{~F}$ & 99 \\
\hline $04223953+1816097$ & LP $415-27$ & 042239.5 & +181609.8 & 54788.18 & M3 & 13.36 & 2100 & $\mathrm{~F}$ & 117 \\
\hline $04420453+1155137$ & LP $475-1747$ & 044204.5 & +115513.8 & 54784.20 & M3 & 13.40 & 2400 & $\mathrm{~F}$ & 95 \\
\hline $04322373+1745026$ & vA 673 & 043223.8 & +174502.7 & 52659.17 & M3 & 13.89 & 300 & $\mathrm{U}$ & 45 \\
\hline $04295572+1654506$ & vA 559 & 042955.7 & +165450.7 & 55770.41 & M3 & 13.07 & 1800 & $\mathrm{~F}$ & 64 \\
\hline $04332699+1302438$ & vA 709 & 043327.0 & +130243.7 & 54786.30 & M3 & 13.39 & 1399 & $\mathrm{~F}$ & 65 \\
\hline $03591350+2025415$ & LP 414-479 & 035913.4 & +202541.6 & 54781.23 & M3 & 13.62 & 2100 & $\mathrm{~F}$ & 89 \\
\hline $04251456+1858250$ & LP 415-794 & 042514.5 & +185824.9 & 54783.23 & M4 & 12.81 & 2400 & $\mathrm{~F}$ & 121 \\
\hline $04175061+1828307$ & vB 250 & 041750.6 & +182830.9 & 54786.25 & M4 & 13.65 & 2699 & $\mathrm{~F}$ & 80 \\
\hline $04290099+1840254$ & vB 278 & 042901.0 & +184025.4 & 54783.26 & M4 & 13.13 & 2400 & $\mathrm{~F}$ & 124 \\
\hline $04311576+1042154$ & RHy 299 & 043115.7 & +104215.5 & 54786.19 & M4 & 14.91 & 4200 & $\mathrm{~F}$ & 61 \\
\hline
\end{tabular}

\section{Notes.}

(a) 2MASS identifiers as in Cutri et al. (2003)

(b) Target names follow the catalogs abbreviated as follows: LP (Luyten 1979), HG (Giclas et al. 1962), Pels (Pels et al. 1975), RHy (Reid 1992), vA (van Altena 1966), vB (van Bueren 1952)

(c) indicates FEROS (F) or UVES (U)

(d) Signal to noise ratio (SNR) is given as the median of several measurements (Stoehr et al. 2007) in wavelength ranges between $8500-8900 \AA$ (FEROS) or $6500-6800 \AA$ (UVES) 



\section{Analysis}

\section{Contents}

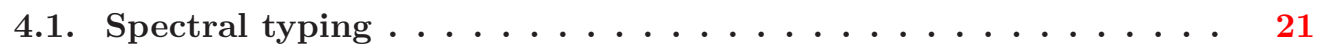

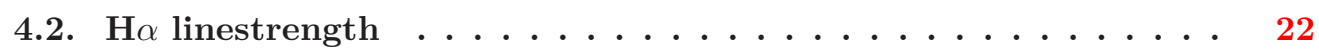

4.3. Coronal X-ray emission . . . . . . . . . . . . . 24

4.4. Rotational velocities . . . . . . . . . . . . 25

4.4.1. Crosscorrelation procedure . . . . . . . . . . . . . 27

4.4.2. Template selection . . . . . . . . . . . . . . . 27

4.4.3. Template matching . . . . . . . . . . . . . . . . . 28

4.4.4. Error estimation . . . . . . . . . . . . . . . . . . 28

4.5. Binary star contamination ... . . . . . . . . 29

4.5.1. Photometric binaries . . . . . . . . . . . . . . . . . 30

4.5.2. Spectroscopic binaries . . . . . . . . . . . . . . . 31

\subsection{Spectral typing}

Many investigations have previously tried to measure stellar rotation and the $\mathrm{H} \alpha$ line spectroscopically. Most of these studies refer to different stars in terms of colour, and rarely have authors used the same colours, or even the same photometric systems. This makes comparison with and analysis of previous measurements a gruelling undertaking. The most intuitive scale rather than colour is a classification by spectral type through effective temperature, and we chose to employ this scheme to further comparableness among the objects under investigation in the present study.

As spectra are not available for the entire sample to derive effective temperatures spectroscopically, a determination has to rely on photometry to ensure the most homogenous classification throughout all objects. Compiled magnitudes from the literature are presented in Table 3.1 for the observed sample. Optical magnitudes or colours for additional stars (combined sample) are taken from the corresponding source papers; additionally, we also infer their 2MASS JHK magnitudes from the NOMAD catalog. Magnitudes and colours of Hyades that were included into the combined sample were originally given in a variety of photometric systems, and need to be transformed appropriately for a direct comparison. Additional difficulties come into play when bolometric magnitudes are desired. As an example, Stauffer et al. (1997a) is based on $V-I$ on the Kron system (Bessell \& Weis 1987), while the original Reid survey of the Hyades (Reid 1992) is drawn from photographic plates in $B-V$, and the more detailed studies that followed originate in observations mostly calibrated onto the Cousins system (Bessell \& Weis 1987) in $R-I$ (Reid 1993). The traditionally used $B-V$ colour saturates in the early M-dwarf regime as the flux in the blue bandpasses vanish, and is of little use. Moreover, accurate $B-V$ data are hardly available for these late type stars as they approach the photometric faintness limit in $B$. However, having NIR magnitudes at 
hand for all of the targets, we opt to base our stellar classification on the homogenous 2MASS photometry.

To convert colours into effective temperatures, we compare $T_{\text {eff }}$ obtained by transformations from different colour indices. Generally, $J H K$ magnitudes have very small uncertainties because they originate from a homogenous, deep survey; and were measured simultanously, so that they do not suffer from partial brightness variations due to stellar activity modulations, and other transient effects. Although $V-K$ is most sensitive to $T_{\text {eff }}$ (smallest slope) and a sensitive temperature indicator, optical $B V R$ magnitudes are (additionally to the above mentioned) somewhat problematic since they were not measured simultanously, but originate from very different epochs than the 2MASS colours. $J-H$ shows a non-monotonic reversal, and $J-K$ is less monotonic and shows a steeper slope and less smoothness around $T_{\text {eff }} \approx$ $3500 \mathrm{~K}$ than $H-K$; we thus opt for the $H-K$ index to convert into effective temperatures and use the colour-temperature calibrations derived by Worthey \& Lee (2011) for solar metallicity cool dwarfs. We find that these calibrations are more complete (in terms of spectral types covered) and more reliable for cool low-mass dwarfs (cf. Fig. 4.1), and rely on a broader sample of stars than individual colour calibrations such as Tokunaga (2000), Kenyon \& Hartmann (1995), or Leggett et al. (2000), which have a sparser sampling in this spectral type regime (there are only relatively few stars available in their calibrations of the "K-dwarf desert") or do not extend at all to cool M-type temperatures. We note that the Tokunaga and Worthey calibrations are in good agreement for $T_{\text {eff }} \gtrsim 3900 \mathrm{~K}(\mathrm{M} 0)$, for lower temperatures the Tokunaga relation yields systematically higher temperatures $(\$ 200 \mathrm{~K}$ for $H-K)$, which we attribute to the variety of sources used for different spectral types therein.

To transform $(H-K)_{\mathrm{BB}}$ (homogenized Bessell \& Brett system, which the Worthey et al. calibrations are based on) into $T_{\text {eff }}$, we fit a 4 th order polynomial,

$$
T_{\text {eff }}=\sum_{i=0}^{4} c_{i}(H-K)_{\mathrm{BB}}^{i}
$$

to the Worthey \& Lee (2011) data from $5500 \mathrm{~K}$ downwards to the lowest available temperatures (thus covering types $\mathrm{K} 0$ to $\mathrm{M} 6$ ), and apply the transformations given in Carpenter (2001) to convert our 2MASS $H-K$ colours into the homogenized system of Bessell \& Brett (1988):

$$
\left(H-K_{s}\right)_{2 \mathrm{MASS}}=(0.996 \pm 0.019)\left(H-K_{s}\right)_{\mathrm{BB}}+(0.028 \pm 0.005)
$$

The effective temperatures in Table A.1 are then computed from the polynomial coefficients listed in Table 4.1. We use an adopted MK-classification after Kenyon \& Hartmann (1995) and Golimowski et al. (2004) to assign spectral types to effective temperatures, given in Table 4.2 for completeness.

Uncertainties in the temperature derivation are dominated by the photometric errors in 2MASS $H$ and $K_{s}$ magnitudes, and by the coefficients in Eq. (4.2), which are of the same order. Errors in $H$ and $K_{s}$ range from $0.020 \ldots 0.046$ and $0.016 \ldots 0.031$, respectively. This translates into a temperature error of $574 \ldots 871 \mathrm{~K}$ and $139 \ldots 224 \mathrm{~K}$ for the hottest and coolest object in our sample, respectively. We thus estimate the uncertainty in temperature to be correct within \pm one spectral sub-types at the hot and cool end.

\section{2. $\mathrm{H}_{\alpha}$ linestrength}

From the extracted spectra in the observed sample, $\mathrm{H} \alpha(\lambda 656.3 \mathrm{~nm})$ equivalent widths were measured as a proxy for chromospheric activity. We express the $\mathrm{H} \alpha$ emission strength relative 

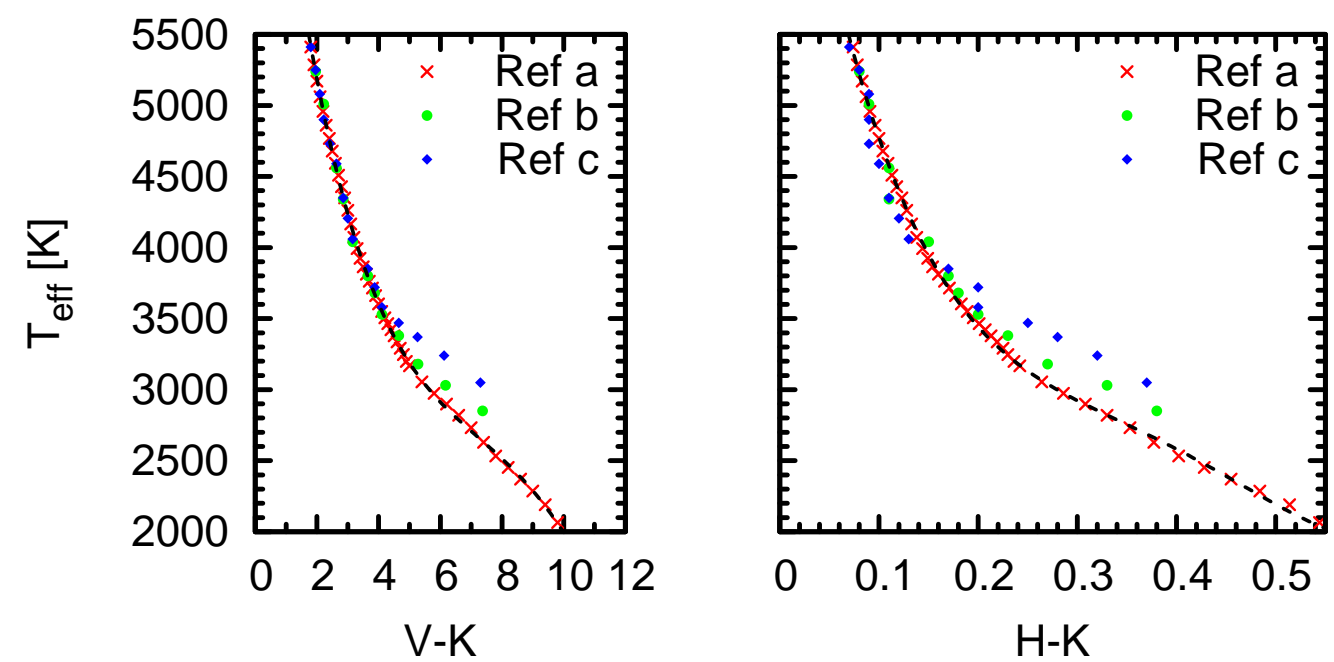

Figure 4.1.: Comparison of colour-temperature calibrations in $V-K$ and $H-K$ colour from Worthey \& Lee (2011); Tokunaga (2000); Kenyon \& Hartmann (1995); denoted as "Ref a" to "Ref c", respectively. The dashed lines are fits to Worthey \& Lee (2011) using a 4th order polynomial.

to the bolometric luminosity, ie. $L_{\mathrm{H} \alpha} / L_{\mathrm{bol}}$ to account for the steeply decreasing luminosity within the spectral range $\mathrm{K}-\mathrm{M}$, which would otherwise overestimate equivalent widths of $\mathrm{H} \alpha$ emission for earlier spectral types. The bolometric flux $F_{\mathrm{bol}}$ is computed from synthetic PHOENIX spectra (Hauschildt et al. 1999) of the same spectral types. The continuum level around the $\mathrm{H} \alpha$ line was determined by fitting the adjacent wavelength regions with a linear function. We adopt $6545-6559 \AA$ and $6567-6580 \AA$ as continuum ranges for all objects, and subtract the fit before determining equivalent widths of the $\mathrm{H} \alpha$ line. We estimate our detection limit by measuring the standard deviation on either side of $\mathrm{H} \alpha$ in the continuum, and take the average to be the noise level above which we can identify $\mathrm{H} \alpha$ absorption/emission. For the earliest spectral types, this yields a limit of around $\log L_{\mathrm{H} \alpha} / L_{\mathrm{bol}} \lesssim-6$, for the latest types the detection limit is somewhat degraded to around $\log L_{\mathrm{H} \alpha} / L_{\mathrm{bol}} \lesssim-5$ owing to the much lower $\mathrm{S} / \mathrm{N}$ in this wavelength regime. We convert the line equivalent widths by scaling them with the absolute flux computed from the corresponding range in the PHOENIX models. Then, the luminosity ratio is given by $\log L_{\mathrm{H} \alpha} / L_{\mathrm{bol}}=\log F_{\mathrm{H} \alpha} / F_{\mathrm{bol}}$. A few objects in the extended catalog that originate from the literature are lacking $\log L_{\mathrm{H} \alpha} / L_{\mathrm{bol}}$, but have only $\mathrm{H} \alpha$ equivalent widths. We estimate their relative $\mathrm{H} \alpha$ luminosities using the reported data together with the effective temperatures and the prescription described above. For vA 115 and

Table 4.1.: Polynomial coefficients used for colour-temperature transformations as in Eq. 4.1.

\begin{tabular}{lrr}
\hline \hline Parameter & Value & Error \\
\hline$c_{0}$ & 7961.32 & $0.8 \%$ \\
$c_{1}$ & -45665 & $2 \%$ \\
$c_{2}$ & 163837 & $4 \%$ \\
$c_{3}$ & -276573 & $6 \%$ \\
$c_{4}$ & 170830 & $7 \%$ \\
\hline
\end{tabular}



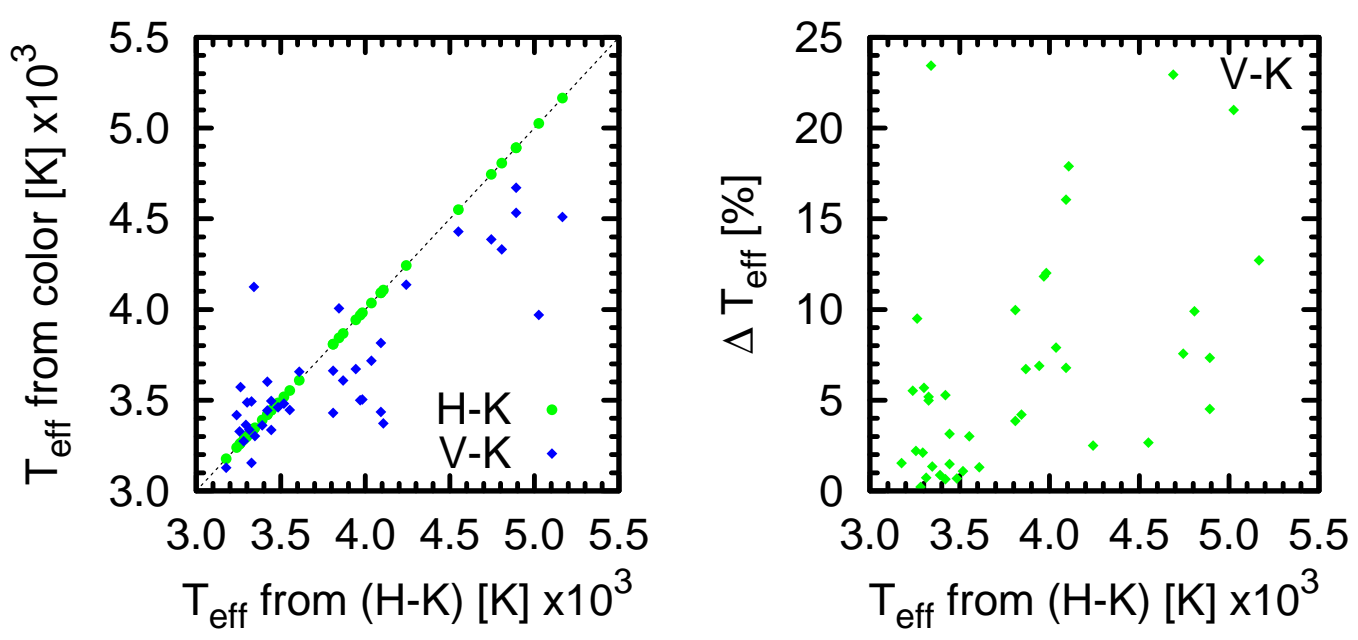

Figure 4.2.: Comparison of effective temperatures obtained for the sample stars from $H-K$ and $V-K$ colour, plotted against temperature (left) and fractional difference

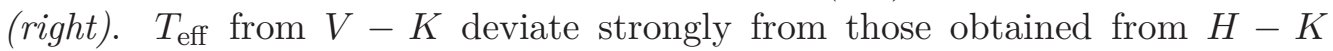
(especially towards the "K-dwarf desert"), given that the $V$ magnitudes are generally of poorer quality and from a multitude of originating photometric systems, whereas $H-K$ are concise 2MASS magnitudes.

vB 190, Stauffer et al. (1997a) measured comparably small equivalent widths of H $\alpha$ absorption/emission. Both objects were later found to be multiple systems (Guenther et al. 2005; Bender \& Simon 2008, respectively), and so $\mathrm{H} \alpha$ is only an average proxy, indistinguishable for the individual stars (cf. Sec. 5.1). We assume that for stars with $\mathrm{H} \alpha$ measured in both small absorption and emission (over several measurements), the detected emission strength is an upper bound for what one can expect to be the normal (non-flaring) activity level. We give upper limits based on the Stauffer equivalent widths also for vA 334, which was reported in moderately strong emission $(0.7-0.95 \AA)$.

\subsection{Coronal X-ray emission}

$\mathrm{X}$-ray continuum emission is an excellent tracer of coronal magnetic activity, complementing indicators from the chromosphere such as $\mathrm{H} \alpha$ and Ca II. Strong X-ray emission has been widely measured in FGK solar-like stars in the solar neighbourhood, particularly facilitated by surveys such as the ROSAT All Sky Survey (RASS). At larger distances and later spectral types, X-ray detections are demanding and often only upper limits can be placed for later type stars. Stern et al. (1995) have conducted an extensive survey of the entire Hyades region based on the RASS, detecting 187 X-ray active Hyades down to spectral type mid M. Other studies (Stern et al. 1994; Pye et al. 1994; Reid et al. 1995) have used deep ROSAT pointed observations to identify Hyades members in X-rays. We cross-match these X-ray detections in the Hyades with our extended sample stars so to provide a complete and concise picture of chromospheric and coronal activity for Hyades $\mathrm{K}$ and $\mathrm{M}$ stars. We do so by correlating our optical positions with their X-ray counterparts in the respective catalogs, finding very good positional agreement for most objects (usually within a few arcseconds). Most of our sample 
Table 4.2.: Spectral type correlation to effective temperatures

\begin{tabular}{cccc}
\hline \hline Spectral type & $\begin{array}{c}T_{\text {eff }} \\
{\left[{ }^{\circ} \mathrm{K}\right]}\end{array}$ & Spectral type & $\begin{array}{c}T_{\text {eff }} \\
{\left[{ }^{\circ} \mathrm{K}\right]}\end{array}$ \\
\hline K0 & 5250 & M0 & 3850 \\
K1 & 5080 & M1 & 3720 \\
K2 & 4900 & M2 & 3580 \\
K3 & 4730 & M3 & 3470 \\
K4 & 4590 & M4 & 3370 \\
K5 & 4350 & M5 & 3200 \\
K6 & 4205 & M6 & 2900 \\
K7 & 4060 & M7 & 2620 \\
& & M8 & 2490 \\
& & M9 & 2400 \\
\hline
\end{tabular}

targets (85\%) are identified among the Stern et al. (1995) detections, where the limiting X-ray luminosity is $1-2 \times 10^{28} \mathrm{ergs} \mathrm{s}^{-1}$ (at the Hyades' distance). Although the detection limit is lowered by about a magnitude $\left(\approx 1.5 \times 10^{27} \mathrm{ergs} \mathrm{s}^{-1}\right)$ by the deeper pointings, eg. Reid et al. (1995), only a small projected cluster area is covered, thus resulting in comparably few detected Hyades. We use the well established conversion factor of $6 \times 10^{-12} \mathrm{ergs} \mathrm{s}^{-1}$ counts $^{-1}$ to obtain X-ray fluxes from published ROSAT countrates. ${ }^{1}$ A number of objects are not detected, these are mostly among the latest spectral types. These stars are probably just too faint to produce an X-ray luminosity above the detection threshold, i.e. the irradiated X-ray flux is below the detection limit of ROSAT at the corresponding distance. For such non-detections, Table A.1 lists upper limits based on the sensitivity of the RASS. Individual non-detections are discussed in Sec. 5.2.2.

Bolometric luminosities are based on individual $T_{\text {eff }}$ and the corresponding luminosity from $\mathrm{BCAH}$ isochrones (650 Myr, solar metallicity). Because the (projected) size of the Hyades cluster extends over many square degrees, and the cluster diameter is of order $20 \mathrm{pc}$ (Röser et al. 2011), the absolute distance to individual cluster stars can vary significantly, thus potentially overestimating the luminosity by a factor of up to 2.5. We try to minimize this effect by obtaining individual distances for all objects where available. Distances are preferentially taken from parallaxes in Röser et al. (2011), and subsequently from Stern et al. (1995) and references therein. In a few cases, no distance information is available, and we assume for these stars a distance of $45 \mathrm{pc}$, eg. the approximate distance to the cluster center $(46.34 \pm 0.27 \mathrm{pc}$, Perryman et al. 1998). Table 3.1 lists the individual distances and their origin for stars in the observed sample.

\subsection{Rotational velocities}

We determine the stellar projected rotational velocity ${ }^{2} v_{\text {rot }} \sin i$ by measuring the spectral line broading. Rotation is degenerate in $\sin i$, where $i$ is the inclination angle between the stellar rotation axis and the line-of-sight $\left(i=90^{\circ}\right.$ for pole-on). It can generally be assumed

\footnotetext{
${ }^{1}$ This factor has been shown, eg. by Fleming et al. (1993); Stern et al. (1994, 1995); Reid et al. (1995), to well describe soft $(\approx 0.1-1.8 \mathrm{keV}) \mathrm{X}$-ray emission from stellar coronae.

${ }^{2}$ To ease readibility, we will use the shortened $v \sin i$ to refer to rotational velocity in the following text. This should not be mistaken as radial velocity.
} 
that $i$ is randomly distributed, and hence does not pose a systematic bias on a large sample, albeit the rotational velocities of individual objects remain lower bounds of the true velocity.

We employ a cross-correlation technique to infer the line broadening similar to the methods used by Browning et al. (2010) and West \& Basri (2009a) for cool, M-type stars. Our method cross-correlates each of the object spectra against a template spectrum of equal or very similar spectral type. The templates are constructed from slowly rotating stars observed with the same instrumental setup to minimize the effects of instrumental profile and spectral resolution changes. All of the templates have measured $v \sin i \leq 3 \mathrm{~km} / \mathrm{s}$ (cf. Table 4.3, and references therein) from high-resolution spectroscopy, which is at or below the detection limit for the $R=48000$ FEROS spectra, and a conservative estimate in case of UVES observations. This means that there are three categories of template and program stars owing to three different combinations of instrument and resolving power. The template stars were observed over a wide range in time, but were treated following the same steps as the object data in Sec. 3.3. We consider the template stars as non-rotating and their spectra as rotationally unbroadened, leaving them with an imprint of instrumental broadening only plus other physical broadening agents (which are negligible in templates and objects for our purposes). Categories of program and templates are thus always matched, i.e. program stars of a given instrument are always treated with template observations of the same instrument and with the same resolving power.

Table 4.3.: Template stars used for the cross-correlation analysis.

\begin{tabular}{|c|c|c|c|c|c|}
\hline Name & $\alpha(J 2000)$ & $\delta(J 2000)$ & $\overline{\mathrm{SpT}}$ & $\begin{array}{c}v \sin i \\
{\left[\mathrm{~km} \mathrm{~s}^{-1}\right]}\end{array}$ & Reference \\
\hline HD 162907 & 175430.1 & -263755 & K0V & 1.0 & Nordström et al. (2004) \\
\hline GL 559B & 143935.1 & -605013 & $\mathrm{~K} 1 \mathrm{~V}$ & 1.1 & Saar \& Osten (1997) \\
\hline Gl 144 & 033255.8 & -092729 & $\mathrm{~K} 2 \mathrm{~V}$ & 3.0 & Nordström et al. (2004) \\
\hline Gl 33 & 004822.9 & 051650 & $\mathrm{~K} 2 \mathrm{~V}$ & 2.0 & Nordström et al. (2004) \\
\hline Gl $105 \mathrm{~A}^{\S}$ & 023604.9 & 065312 & K3V & 2.0 & Nordström et al. (2004) \\
\hline Gl 542 & 141904.9 & -592244 & $\mathrm{~K} 3 \mathrm{~V}$ & 1.0 & Nordström et al. (2004) \\
\hline $\mathrm{Gl} 845 \mathrm{~A}$ & 220321.7 & -564709 & $\mathrm{~K} 4.5 \mathrm{~V}$ & 2.0 & Nordström et al. (2004) \\
\hline Gl 664 & 171613.4 & -263246 & $\mathrm{~K} 5 \mathrm{~V}$ & 2.0 & Nordström et al. (2004) \\
\hline Gl 9251B & 080709.1 & 072300 & $\mathrm{~K} 7 \mathrm{~V}$ & 1.4 & López-Santiago et al. (2010) \\
\hline Gl 57 & 012134.6 & -413923 & $\mathrm{~K} 7 \mathrm{~V}$ & 3.0 & this work \\
\hline Gl 229 & 061034.6 & -215146 & M0.5 & 1.0 & Reiners (2007) \\
\hline Gl 514 & 132959.0 & 102246 & M0.5 & 1.5 & Reiners (2007) \\
\hline Gl 2 & 000510.2 & 454712 & M1.0 & 2.5 & Browning et al. (2010) \\
\hline Gl 908 & 234911.9 & 022411 & M1.0 & 2.5 & Browning et al. (2010) \\
\hline Gl 173 & 043741.9 & -110218 & M1.5 & 2.5 & Browning et al. (2010) \\
\hline Gl 205 & 053126.9 & -034022 & M1.5 & 1.5 & Reiners (2007) \\
\hline Gl 433 & 113527.1 & -323208 & M1.5 & 2.5 & Browning et al. (2010) \\
\hline Gl 70 & 014320.3 & 041923 & M2.0 & 2.5 & Browning et al. (2010) \\
\hline Gl 411 & 110322.3 & 355720 & M2.0 & 2.5 & Browning et al. (2010) \\
\hline Gl 84 & 020504.1 & -173651 & M2.5 & 3.0 & Reiners et al. (2012) \\
\hline Gl 581 & 151927.5 & -074320 & M3.0 & 2.5 & Browning et al. (2010) \\
\hline
\end{tabular}

Notes. ${ }^{\S}$ denotes template spectra available from both FEROS and UVES. All other template stars from either instrument. 


\subsubsection{Crosscorrelation procedure}

Each template spectrum is artificially (rotationally) broadened employing a variable linebroadening kernel (Gray 2005) that corresponds to a series of rotational velocities from 0 to $50 \mathrm{~km} / \mathrm{s}$. As the broadening is non-linear, step sizes range from $0.5 \mathrm{~km} / \mathrm{s}$ to $5 \mathrm{~km} / \mathrm{s}$ at the lower and upper ends, respectively. These are then each cross-correlated with the original, unbroadened template. The resulting cross-correlation functions (XCFs) are a series of distinct gaussian-like peaks, where their width corresponds to the broadening applied to the template spectrum. We fit these peaks with gaussians and measure their FWHM. The correlation function's width as a function of the underlying rotational velocities used in the broadening kernel yield a broadening "growth curve" that is specific for each combination of object and template spectra (and also depends on the wavelength range). This growth curve enables the computation of $v \sin i$ inherent to a spectrum from the width of its crosscorrelation profile. Cross-correlating the object spectrum with the unbroadened template $(v \sin i \approx 0 \mathrm{~km} / \mathrm{s}$ ) and measuring the corresponding width of the correlation function thus gives the rotational velocity $v \sin i$ of the object. Note that template and object spectra have the same instrumental profiles, and are of very similar spectral type so that other linebroadening mechanisms than $v \sin i$ are negliable and do, at most, alter the correlation profiles in a systematic way.

For each object-template combination, $v \sin i$ is measured from a set of pre-selected wavelengthranges. These are typically $20-50 \mathrm{~nm}$ wide and exclude ranges with telluric features identified in the atlases of Kirkpatrick et al. (1991) and Wallace et al. (2011). Cross-correlation is performed in about 20 ranges between $\lambda \lambda 500-900 \mathrm{~nm}$ that contain moderately deep, unblended photospheric lines. We exclude overly broad and deep lines as these dominate and smear out the correlation profiles. Similarly, molecular band-heads are omitted, as these can produce a correlation profile with many peaks due to the many narrowly spaced lines in the spectrum. Correlation profiles that do not resemble a gaussian are not taken into account. Each profile is assigned a quality flag, and only the best fits are considered in the further analysis. The shape of the correlation profile may also help to discriminate binary stars.

\subsubsection{Template selection}

Our template stars are field dwarfs, and thus probably considerably older than the intermediate aged Hyades (625 Myr, Perryman et al. 1998). The latter have settled on the main sequence, but show quite some differences in their spectra when compared to the field stars of the same spectral type. This is likely due to $\log g$ contraction effects, and results in subtle differences e.g. in linestrengths. The transition from K-type to M-type stars is particularly affected by a somewhat variable onset of dominating molecular features ( $\mathrm{TiO}, \mathrm{FeH}$ ), which occurs in the open cluster stars at a spectral type that may be one or two sub-types earlier or later compared with the field stars. To account for the different spectral features present in early K to mid M-type stars, we use different sets of wavelength-ranges for K- and M-stars. Furthermore, each cross-correlation analysis of an object is performed with at least three templates, where the additional templates are of neighbouring spectral types. We find that this scheme reduces the uncertainty in the $v \sin i$ determination significantly, and gives us an additional measure of the suitability of a specific template for a given object.

Our sample of Hyades is comprised of the full range of $\mathrm{K}-$ mid M spectral types. This broad range makes the selection of suitable template stars a somewhat delicate issue, since the spectral shape and abundances drastically change over the course of $\mathrm{K}$ to M-types, rendering 
a comparison of even close spectral types difficult. Other studies of rotation, mostly in field stars, avoid this issue by focussing solely on a narrow, more homogenous range of spectral classes, e.g. either a few adjacent spectral sub-classes in the M-type regime or earlier than mid K-type stars. The performance of the cross-correlation method to determine $v \sin i$ to a certain degree depends on a good match between object and template spectrum. For our sample objects, it is obvious that a single template star cannot be employed for the entire set of object spectra. Our approach is to optimize the spectral difference between object and template, which in our experience provides the most robust results. We carefully constructed a set of template stars for both the FEROS and UVES subsamples independently, aiming at the slowest possible rotators among the range of spectral types covered. Only a few template stars are observed with both instruments. For the UVES subsample, an additional challenge is to match wavelength coverage and instrumental mode of object and template, since UVES observations were performed in a variety of resolving powers and wavelength settings. The resulting set of templates is listed in Table 4.3.

\subsubsection{Template matching}

We were unable to follow our strict methodology for six program stars observed with UVES:

1. For 2M 03202921+0827161 (HIP 15563), 2M 04094935+0918197 (HIP 19441), and for 2M $05004888+0443591$ (HIP 23312) the appropriate template spectra are only available at higher resolution compared to the targets. We degraded the corresponding templates using a gaussian-like instrumental broadening kernel (Gray 2005) to match the resolution of the objects.

2. For 2M 04291234+1516259 (vA 529), the same procedure was applied, but degrading the object spectrum to match the available templates.

3. Observations of 2M 04033902+1927180 (RHy 44) and 2M 04322373+1745026 (RHy 308) were performed under conditions with the spectrograph's slit width larger than the seeing, so that the spectral resolution is not slit width but seeing dominated. In these cases, we degraded the resolution of the template spectra for each object-template combination following two steps: first, we measured the width of unblended telluric lines in the $\mathrm{O}_{2}$ band $6237-6350 \AA$ in the objects by fitting gaussian profiles (these lines are free from stellar broadening agents). Then, we instrumentally broadened the corresponding template spectra (available in higher resolution) until the template's telluric linewidths match those in the objects. In doing so, we assume that the intrinsic $\mathrm{O}_{2}$ linewidths of the program star's observation are negligible compared to the effect of seeing $\left(0.66^{\prime \prime}\right.$ and $1.15^{\prime \prime}$ for the two objects, respectively). The resulting object-template combinations then underwent the usual cross-correlation analysis.

\subsubsection{Error estimation}

The final (projected) rotational velocity for an object is then computed as follows: From the set of $v \sin i$ values obtained from the different wavelength-ranges for one template, those with a low quality flagged profile are discarded. For the remaining, the median and the standard deviation are taken. A typical spread is on the order of $1 \mathrm{~km} / \mathrm{s}$. From all template spectra employed for an object, a weighted average is computed from the individual median values, whose error we consider as the formal $v \sin i$ error, given in Table A.1. We note that this error 
reflects the differences between the individual wavelength-ranges for each template spectrum, but is not strictly tied to the physical rotational velocity of the program star. The formal error should therefore be taken as a guide of relative uncertainty rather than as absolute numbers. Following Mohanty \& Basri (2003), we estimate that random errors are around $\pm 1 \mathrm{~km} / \mathrm{s}$, and that errors introduced by instrumental broadening at our resolution are marginal. The true rotational velocity for individual stars is therefore probably good within $\pm 1 \mathrm{~km} / \mathrm{s}$ to $\pm 2 \mathrm{~km} / \mathrm{s}$ (albeit the degeneracy in inclination $i$ still remains). We determine the detection limit in $v \sin i$ of our method by analyzing the cross-correlation growth curve. This function is smooth until a steep cut-off towards zero rotational broadening, where artifical broadening of the slow template spectrum has little to no effect on the width of the XCF. We empirically determine the location of the cut-off by fitting a linear function to both sides of the knee, the intersection then gives an estimate of the minimum rotational broadening kernel for which we can measure the width of the resulting XCF. For most object-template combinations, this happens around $2 \mathrm{~km} / \mathrm{s}$ to $2.5 \mathrm{~km} / \mathrm{s}$, and is naturally at slightly lower values for the UVES observed program stars with the highest resolving power. However, the intrinsic rotational broadening of our template stars is not zero, but usually determined by an upper limit of 2.5 to $3.0 \mathrm{~km} / \mathrm{s}$. Ideally, such a calibrator should be non-rotating, which for obvious reasons is not feasible. Basri et al. (2000) showed that rotating template stars are appropriate as long as the targets are rotating faster than the template. If the target star has $v \sin i$ below that of the template, this can be determined, though not the exact $v \sin i$ itself. The template stars chosen for our purpose are among the slowest rotators available in the given spectral type range, so that our $v \sin i$ determination is expected to be limited by the least slowest rotators among them. We thus conservatively estimate our detection limit in $v \sin i$ to be $3.0 \mathrm{~km} / \mathrm{s}$, corresponding to the upper bound of intrinsic $v \sin i$ of the template stars employed (Table 4.3). We apply this limit to all stars in the observed sample, ignoring the fact that individual cases, where template spectra with $v \sin i$ lower than $3.0 \mathrm{~km} / \mathrm{s}$ are applied to the higher resolution observations $(R>50000)$ may yield substantially lower detection limits.

Rotational velocities compiled from the literature, that are included in our extended sample, are given with their corresponding detection limits. Stauffer et al. give a limit of $6 \mathrm{~km} / \mathrm{s}$ for their measurements, while Reid \& Mahoney (2000) quote a limiting $v \sin i$ of $2.5 \mathrm{~km} / \mathrm{s}$ following a similar, but simplified cross-correlation method on their observations obtained at $R=45000$ (essentially, only two wavelength-ranges are used for the XCF with two fixed template spectra of the same spectral type). Where our observed sample covers stars with previously measured $v \sin i$, this is discussed in Sec. 5.3.2.

\subsection{Binary star contamination}

A multiple stellar system can mimick the effect of rotational broadening. When observed at an unfortunate epoch, the radial velocity shift between the system's components may be just large enough to broaden the spectral line profiles, but small enough not to allow to disentangle the compound line profiles. This implies that the luminosity difference of the stars is rather small, otherwise the contribution from the fainter component fades out quickly. In the latter case, the XCF will be distorted with small sidelopes in the XCF wings instead of an approximately symmetric growth of the entire XCF. It is thus important to estimate the fraction of binary stars that potentially impairs the number of stars with presumably enhanced rotation. The classical approach to identify spectroscopic binaries is of course to search for periodic radial velocity changes. This requires multiple epochs of observations 
of each object, and we do not have repeated observations available. However, also a single epoch observation and its XCF from the cross-correlation analysis are indicative of stellar multiplicity, as are colour-magnitude diagnostics.

\subsubsection{Photometric binaries}

Fig. 4.3 plots the program stars in a colour-magnitude diagram, alongside with an $650 \mathrm{Myr}$ isochrone with solar metallicity, which is the closest available to the Hyades estimate of $[\mathrm{Fe} / \mathrm{H}]=0.14 \pm 0.05$ (Perryman et al. 1998). For masses smaller than $0.6 M_{\odot}$ we use the BCAH set of models from Baraffe et al. (1998), together with the Baraffe et al. (2002) set for larger masses, with the CIT magnitudes from the isochrone models converted into the 2MASS photometric system to match our data by using the transformations in Carpenter (2001). Röser et al. (2011) find that the BCAH models show better agreement in the low mass regime for the Hyades than other models, albeit the low mass end of the Hyades main sequence is sparsely populated by observations.

In Fig. 4.3, we plot in circles stars with absolute magnitudes derived from individual distances, i.e. where parallax measurements are available (cf. Table 3.1), with zero space absorption. The average error from these parallaxes results in a distance uncertainty of about $2 \mathrm{pc}$ for the late type stars covered here. For stars that lack a parallax measurement, we assume the distance to the cluster center ( $45 \mathrm{pc}$, starred symbols in Fig. 4.3). The Hyades are very extended in their spatial structure, with a derived tidal radius of $r_{t} \approx 10 \mathrm{pc}$ (Perryman et al. 1998; Röser et al. 2011, with an enclosed tidal mass of $276 M_{\odot}$ ). They find the contributing mass in the volume outside of $r_{t}$ up to a distance of $30 \mathrm{pc}$ around the cluster center ("halo" and beyond) to be $52 \%$ of the tidal mass. At the same time, the average mass per star decreases from $1.4 M_{\odot}$ in the cluster center to $0.5 M_{\odot}$ at $r_{t}$. Hence, it is more likely to find low mass stars outside of the cluster core, so that we can expect our targets to have distances largely varying around outside the cluster center distance.

In Fig. 4.3, for guidance, the thin solid and dashed lines represent distances of $1 r_{t}$ and $2 r_{t}$, respectively, that a star would end up on if we moved it by that distance along the line of sight. The minimum and maximum radial distances of cluster members is, for comparison, $19 \mathrm{pc}$ and 75 pc. Fig. 4.3 suggests that the photometry follows the general trend of the model well, with larger scatter towards the redder part $\left(H-K_{s}>0.2\right.$, i.e. spectral type M3). We estimate the displacement in the CMD of potential binaries by constructing isochrones for binary systems with different types of secondary stars. A mass ratio of 1:1 (green long-dashed) and effective temperature differences of $\Delta T_{\text {eff }}=300,700$, and $1500 \mathrm{~K}$ between primary and secondary are displayed (dashed, dashed-dotted, dashed double-dotted, respectively). Several aspects can be readily seen: Our binary isochrones are rather meant as an illustration, yet there are only a handful of stars brighter in $M_{K_{s}}$ than prescribed by the equal-binary isochrone. This motivates a literature search to identify published binaries, which we plot as filled symbols. Most stars in this bright regime turn out to be multiple, so that we flag the remaining seven objects outside of the equal-binary isochrone and in the range $4<M_{K_{s}}<7.5$ as suspicious. Given the photometric dispersion along the MS, this is only a very subtle indication of potential multiplicity.

Two objects in the lower left of Fig. 4.3 are peculiar. 2M 04270642+1625482 (RHy $240 \mathrm{AB}$ ) is among the faintest objects, and an HST resolved binary (Gizis \& Reid 1995). Its 2MASS photometry is noted as very bad in the Cutri et al. (2003) database, which explains the misplacement in the CMD. We estimate the individual $T_{\text {eff }}$ by evaluation of the separate $M_{I}$ for the A and B components in Gizis \& Reid (1995). Using the isochrone model, we 


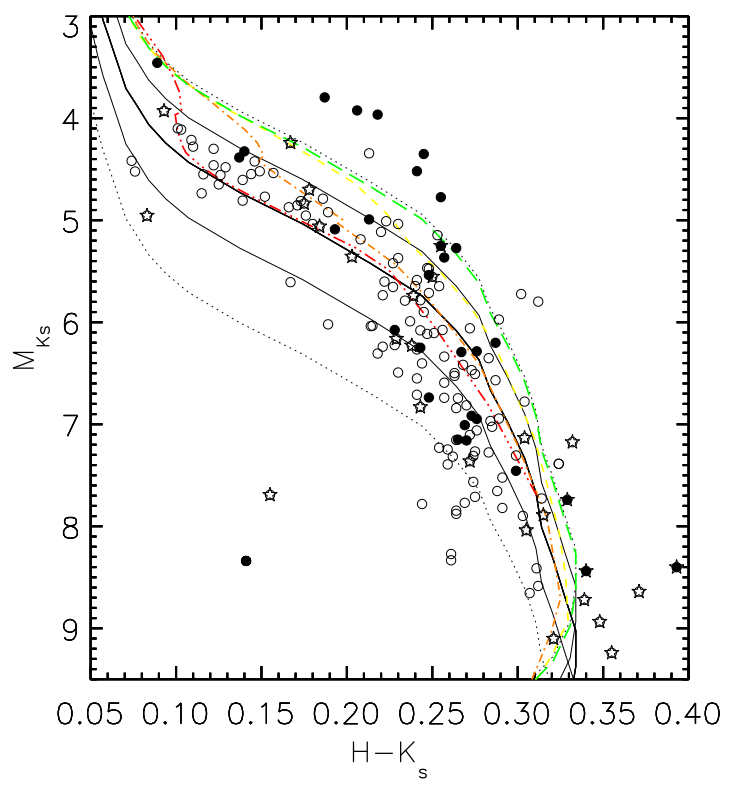

Figure 4.3.: Colour vs. absolute magnitude diagram for selected objects. An isochrone from the BCAH models (Baraffe et al. 1998, 2002) for the Hyades' age (thick solid line) is included. Isochrones corresponding to $r_{t}= \pm 10 \mathrm{pc}$ (tidal radius) and $\pm 20 \mathrm{pc}$ (cluster halo) away or in front of the true cluster center are indicated by thin solid and thin dashed lines, respectively. Other thick curves are derived isochrones for binary stars with a difference in effective temperature of the primary of $0 \mathrm{~K}$ (same mass; long dashed, green), $300 \mathrm{~K}$ (dashed, yellow), $700 \mathrm{~K}$ (dash-dotted, orange), and $1500 \mathrm{~K}$ (dash-double-dotted, red). Starred symbols assume a distance of $45 \mathrm{pc}$, filled symbols mark identified binary systems. See text for details.

predict a spectral type of M5 for this equal mass binary. The second obvious outlier is 2M 03583070+2715238 (RHy 9). Its NIR colours and its brightness suggest it to be a distant background star, however, Reid (1993) finds it a high probability cluster member based on proper motions. Optical colours place it towards slightly redder type between M0 $(B-V)$ to M6 $\left(V-I_{c}\right)$, so that rather the photometric quality than a much larger distance is the reason for its misplacement in the CMD. Recent NIR photometry (Lawrence et al. 2012) from the UKIRT Infrared Deep Sky Survey, transformed into the 2MASS photometric system using the relations in Hewett et al. (2006), give a consistent $M_{K_{S}}=7.55$ and $H-K_{S}=0.305$, placing RHy 9 directly onto the BCAH isochrone as an M5 dwarf, and as such it is kept in our catalog.

\subsubsection{Spectroscopic binaries}

For objects where we have obtained new spectroscopy, we check our single epoch observations for double-lined signatures of SB2 binaries. This procedure is sensitive to binaries with significant radial velocity difference only, and requires that the flux contribution from both stars is of similar strength. Under such conditions, spectroscopic binaries can be easily detected by their double structured XCF in the cross-correlation analysis. We find clear evidence for SB2s in five spectra, which are discussed in Sec. 5.1.

Some stars show $\mathrm{H} \alpha$ line profiles with a double peak, which may mimick the presence of a 


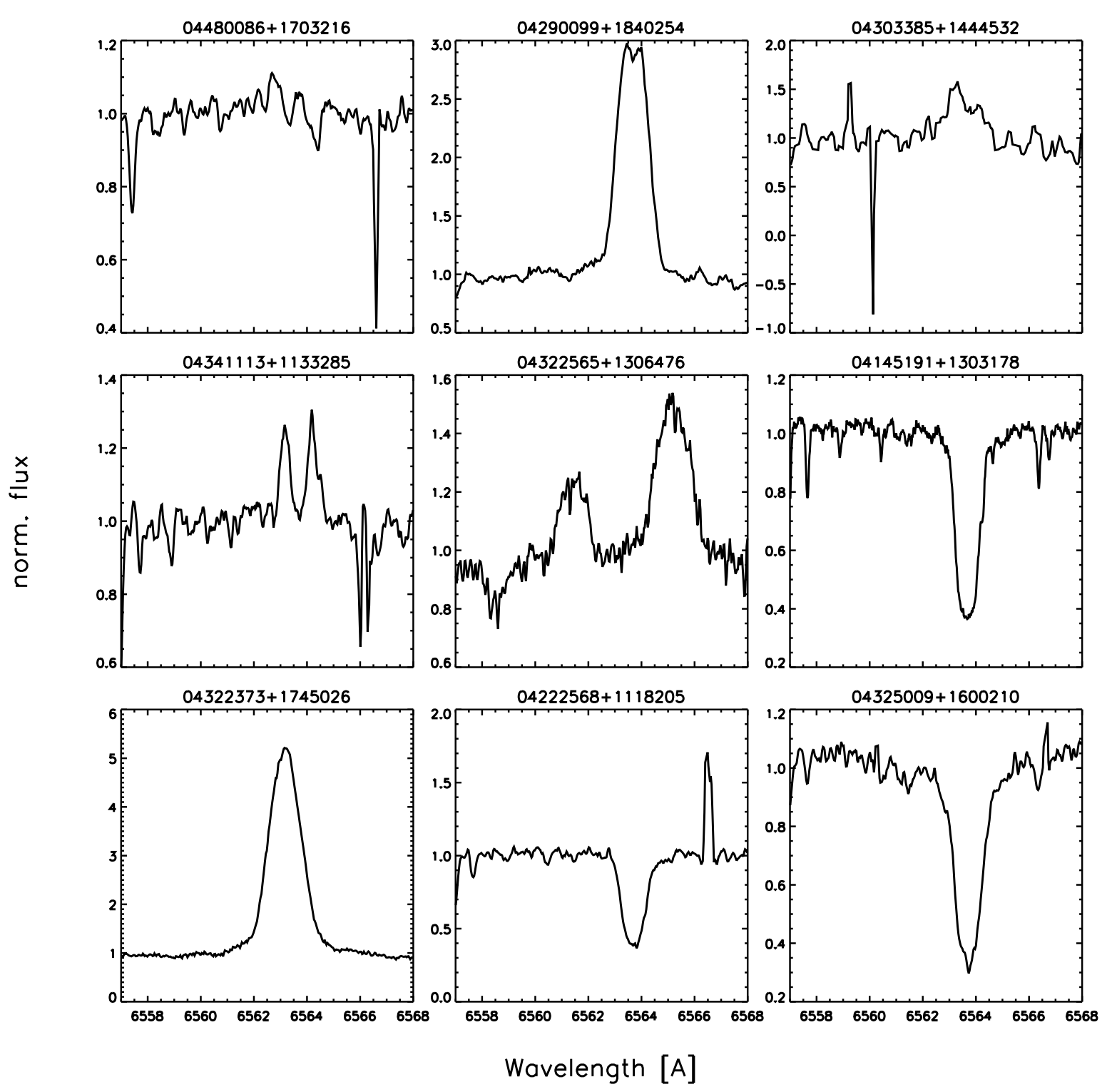

Figure 4.4.: $\mathrm{H} \alpha$ profiles of confirmed binaries among our new spectroscopic observations. Objects are labelled by their 2MASS identifiers. Double lined $\mathrm{H} \alpha$ profiles are readily visible in three of the nine objects

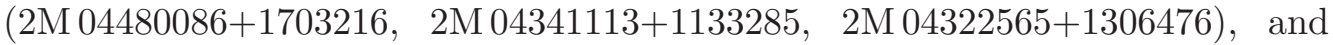
probably also in $2 \mathrm{M} 04303385+1444532$.

companion, or be due to self-absorption in the core of emission line profiles. However, their $\mathrm{XCFs}$ do not support the presence of a companion. We cannot exclude companions with much lower mass though, as such a XCF will only be slightly distorted on one side in the wing before the effect becomes undetectable.

An extensive literature search reveals that a small number of the program stars have multiepoch RV measurements, and in a few cases resolved imaging. We find that 33 objects from our list are known binaries, with a few additional putative detections, listed in Sec. 5.1. Fig. 4.4 displays the spectra around $\mathrm{H} \alpha$ of nine confirmed binaries in our observed sample. 


\section{Results}

\section{Contents}

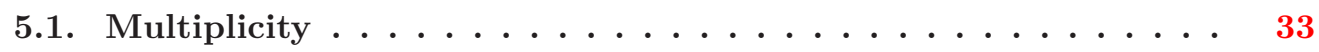

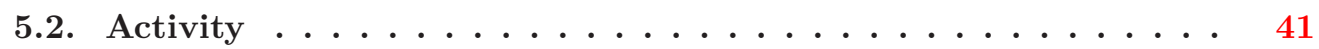

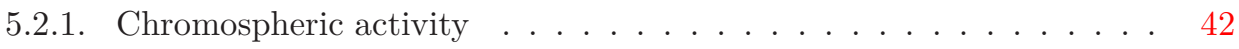

5.2.2. Coronal activity . . . . . . . . . . . . . . 44

5.2.3. Activity fraction . . . . . . . . . . . . . . . 47

5.3. Rotational velocities . . . . . . . . . . . . . 50

5.3.1. Fraction of rotators . . . . . . . . . . . . . . 51

5.3.2. Comparison to previous results . . . . . . . . . . . . 51

5.3.3. Rotation-Activity relation . . . . . . . . . . . . . 53

We have established a large sample of over 170 Hyades low mass stars of spectral types $\mathrm{K}-\mathrm{M}$ in order to illucidate the interconnection of rotational velocity and magnetic activity. The sample of single and multiple stars covers the transitional spectral region where stars become fully convective, and thus enables to test the rotation-activity relation and magnetic braking, known from low mass field stars, for the age of the Hyades open cluster.

\subsection{Multiplicity}

Out of 171 objects, we find 33 of them to be multiple stars with published information in the literature. Among them are seven of the 66 stars that we obtained spectra of.

One of them, 2M J04480086+1703216 (LP 416-43, Pels 95), first noted by Griffin et al. (1985), was probably observed close to orbital phase $\pi / 2$ (maximum projected separation), as it is clearly visible as a SB2 in our spectrum with well separated, unblended spectral line systems of almost equal strength. This indicates similar luminosities, hence spectral types for both components. The XCF is clearly separated into two peaks, and the $\mathrm{H} \alpha$ profile appears split, with a bluer feature in either weak emission or absorption, whereas the redder part is in slight absorption (the $\mathrm{H} \alpha$ line alone could also indicate a single strong absorption profile almost entirely filled-in with emission, if this star would not show a clear SB2 signature). With such a large $\Delta R V$ between the primary and the secondary in LP 416-43, our initial attempt to measure the rotational velocity with the established template stars (Section 4.4) naturally failed, as the separation of the line systems in these spectra is much larger than the line widths. As a result, the XCFs show two distinct peaks, separated by $\Delta \mathrm{RV}$, which could be used separately to determine $v \sin i$. However, we find that this approach is hampered by the small difference in amplitude in the intermediate range between the two peaks in the XCFs, which effectively limits our ability to properly fit the width of the peaks (and hence, $v \sin i$ ). Instead, we employ a binary template approach to measure $v \sin i$ for this SB2 as follows: first we measure the radial velocity separation of the components from the above XCFs. We then clone and superimpose the appropriate template stars with the measured 
$\Delta \mathrm{RV}$, to construct artificial binary spectra. This procedure seems reasonable given that both components in LP 416-43 have nearly identical spectral line depths, so that the spectral type of the composite template spectra should be a good match for both the primary and the secondary. The resulting XCFs have well defined single peaks, and the corresponding growth curves are smooth and give very consistent $v \sin i$ throughout all spectral ranges and for all templates employed.

This approach is susceptible to systematic errors if an incorrect $\Delta \mathrm{RV}$ is applied, because then the error in radial velocity difference propagates into an erronous width of the corresponding XCFs. As a consequence, the rotational velocity will be overestimated. To quantify this effect, we vary the previously determined $\Delta R V$ when constructing the template and evaluate the corresponding rotational velocities. We estimate from fitting the peak positions in the XCFs, that the relative radial velocities can be measured to within better than $\pm 0.5 \mathrm{~km} / \mathrm{s}$, so the error in $\Delta R V$ should never sum up to more than $\pm 1 \mathrm{~km} / \mathrm{s}$. By analyzing the XCFs and obtaining $v \sin i$ for a number of binary templates synthesized with different RV separations within $\Delta \mathrm{RV} \pm 1.5 \mathrm{~km} / \mathrm{s}$, we obtain a function that, when fitted with eg. a gaussian, yields a minimized $v \sin i$ and gives the error budget that can be expected. The smallest $v \sin i$ then corresponds to the best-matching $\Delta \mathrm{RV}$ for the template creation, and evaluation of this function at the estimated uncertainties of $\pm 1 \mathrm{~km} / \mathrm{s}$ in $\Delta R V$ gives the corresponding error in the measured $v \sin i$. In the case of LP 416-43, we get a typical error of $0.5 \ldots 1.5 \mathrm{~km} / \mathrm{s}$ for a single template. This is of the same order as the random errors in the normal treatment, so that systematics resulting from an imperfect creation of these special binary templates should be well controlled and do not significantly degrade the determination of rotational velocity. We note that the resulting $v \sin i$ following the ascribed approach gives, strictly speaking, individual rotation rates for each component in the binary star only if both stars actually have the same $v \sin i$ (eg. if they are tidally locked). If one component is rotating at a different rate, the measured $v \sin i$ does not reflect this but represents some sort of average of the true rotation rates ${ }^{1}$. However, as in LP 416-43 both spectral line systems are alike and of the same depth, there is no reason why in this binary the component should have vastly different (projected) rotational velocities, and thus the derived $v \sin i$ seem robust.

Our analysis shows the opposite behaviour in the case of $\mathbf{2 M ~ J 0 4 3 2 5 0 0 9 + 1 6 0 0 2 1 0}$ (vA 684, vB 91): Griffin et al. (1988) established this object as a binary system with a period of $>15$ years, derived from long-term radial velocities. It is noted as a single-lined spectroscopic binary (SB1), composed of two K stars of similar spectral type (Barrado y Navascues \& Stauffer 1996, derived from photometric deconvolution). We confirm this in the sense that we do not see any spectroscopic hints of a secondary, and our XCFs are well symmetric without typical deformations of a secondary star. $\mathrm{H} \alpha$ is in absorption and does not show any signs of blending or line core emission, so that we regard the contribution of a presumably wide companion star to $\mathrm{H} \alpha$ and $v \sin i$ as negligible.

$\mathbf{2 M ~ 0 4 3 2 2 5 6 5 + 1 3 0 6 4 7 6}$ (vA 677) displays two clearly distinct $\mathrm{H} \alpha$ emission lines, but is otherwise not double-lined. This is interesting since the two strong $\mathrm{H} \alpha$ lines alone indicate $\Delta R V=171 \mathrm{~km} / \mathrm{s}$, which would be easily detectable in the XCF, but we do not notice this in our XCFs. Bender \& Simon (2008) have concluded from RV data a K3-M0 binary, and so we think that this difference in spectral type (hence, luminosity) is the reason for our nondetection, even more so as we find this object to be a fast rotator $(>20 \mathrm{~km} / \mathrm{s})$. The amount of flux from an M0 dwarf is about four times lower than that from a K3-type dwarf, so that the

\footnotetext{
${ }^{1}$ This is because in our approach, both components in the constructed template spectra are treated in the same way, meaning both components have the same rotational broadening apllied.
} 
fainter spectral imprint may be on the verge of detectability, moreover when a high rotation rate dampens the line widths. It is then unlikely that the reduced flux contribution of the M0 companion could dilute the XCFs in a way that could mimick as high an enhanced rotational broadening, hence the large $v \sin i$. As we cannot disentangle the individual rotation, we account for the total $\mathrm{H} \alpha$ emission in our derived $\log L_{\mathrm{H} \alpha} / L_{\mathrm{bol}}=-3.96$, which should be taken accordingly. The emission flux as visible from the spectrum in Fig. 4.4 is not equally shared by both lines; around $64 \%$ can be attributed to the more redward component. As one emission line is likely produced by the lower-mass companion, its true flux remains unknown because its continuum level is outshone by the more luminous star. The compound system appears as of type K4, and we list it as such.

2M J04322373+1745026 (VA 673, HZ 9, WD 0429-176) requires special attention. Röser et al. (2011) includes it as a Hyades member. VA 673 is a known post common envelope binary system (PCEB) with a $0.564 \mathrm{~d}$ period, composed of a DA2.5 white dwarf and a dMe main sequence companion (Lanning \& Pesch 1981). It is the only white dwarf in our sample of Hyades, given that its low-mass companion fits well into our survey. Schreiber \& Gänsicke (2003) derived $T_{\text {eff }}=17400 \mathrm{~K}$ and quote a mass of $0.51 \mathrm{M}_{\odot}$ for the primary, and $0.28 \mathrm{M}_{\odot}$ for the secondary star. O'Dwyer et al. (2003) measured hard X-ray emission $\left(0.5-2.4 \mathrm{keV}, \log L_{X}=28.6 \mathrm{ergs} \mathrm{s}^{-1}\right)$ from the dMe companion; Stern et al. (1995) report $\log L_{X}=28.4 \mathrm{ergs} \mathrm{s}^{-1}$ in their Table 1. Soft X-ray emission is expected from white dwarfs, but hard X-ray emission (greater than $0.5 \mathrm{keV}$ ) in such systems is generally attributed to the companions, with $L_{X}$ consistent to what is seen in field dM stars. Previously, Bleach et al. (2002b) have determined the projected rotational velocity of the dMe companion by measuring the rotational broadening of the $\mathrm{NaI}$ doublet $(8183,8195 \AA)$ and nearby $\mathrm{Fe}$ and $\mathrm{Ti}$ lines (8374-8400 $\AA$ and $8410-8430 \AA$ ). They find a $v \sin i$ between $31.0 \pm 7.0$ and $40.0 \pm 8.5 \mathrm{~km} \mathrm{~s}^{-1}$ with two different template stars (cf. Table 6 in Bleach et al. 2002b). The $v \sin i$ from the $\mathrm{Na}$ I doublet is, for both templates, considerably higher (though within the error bars). The authors identify an imperfect telluric line removal as a source of a possible overestimation, as the Na I doublet is severely contaminated by tellurics. With our methodology, we find $v \sin i=23.6 \pm 0.8 \mathrm{~km} / \mathrm{s}$. This is considerably lower (by 24\%) than the lowest value reported by Bleach et al. (2002b), $31 \pm 7 \mathrm{~km} / \mathrm{s}$, and just within the error margins. We use spectral windows in the range 6131-6800 $\AA$ (telluric line contamination by $\mathrm{O}_{2}$ and $\mathrm{H}_{2} \mathrm{O}$ excluded (Wallace et al. 2011) as described in Sec. 4.4), hence our measurements are not prone to possible telluric line removal issues. We obtain consistent values throughout all employed template stars, and the XCFs are adequately gaussian without obvious deformations from the primary star. Although the flux contribution from the DA white dwarf is still $\approx 20 \%$ contiunuum flux by $\mathrm{H} \alpha$ (from a blackbody simulation), essentially all spectral lines (except broad hydrogen and telluric lines) originate from the companion star. Thus, the inferred $v \sin i$ in this binary system is measured solely for the companion star, and should not be diluted by line-broadening of the primary white dwarf. For interactions between primary and secondary star in this system and its implications on rotation, refer to Sec. 6.

The spectrum of VA 673 (Fig. 4.4) shows strong evidence of magnetic activity. Bleach et al. (2002a) have noted a very high level of quiescient chromospheric activity, and in our spectrum a multitude of atomic lines $\left(\mathrm{H} \alpha-\mathrm{H}_{\eta}\right.$, He I $\mathrm{D}_{3}$, Na I D, Ca II IRT, and others) are observed in emission. We obtain $\log L_{\mathrm{H} \alpha} / L_{\mathrm{bol}}=-3.46$ for the dM companion of VA 673 , which makes it the most active star in our observed sample. The system also shows enhanced X-ray emission of $\log L_{X} / L_{\mathrm{bol}}=-3.17$. The flux contribution of the white dwarf is about $1.7 \%$ and $1.2 \%$ at the $H$ and $K$ band centers, respectively. This is only $95 \%$ (75\%) of the 2MASS photometric errors in $H\left(K_{s}\right)$ for VA 673, so that our determination of spectral type from $H-K$ should 
not be compromised. The derived M3 spectral type for the low mass companion in VA 673 is in agreement with the type obtained by Hoard et al. (2007), also from photometry.

VA 673 is a PCEB system, i.e. the companion has been tidally locked during the common envelope phase and is left as a fast rotator. As a result, a high level of magnetic activity can be expected to be generated by this rotation. Our results for VA 673 demonstrate that this is indeed the case, with strong $\mathrm{H} \alpha$ and X-ray emission. Recently, Rebassa-Mansergas et al. (2012) have surveyed a larger sample of PCEB systems, demonstrating that enhanced activity is seen in all close white dwarf/dM binaries following this mechanism, and we confirm this picture for the Hyades PCEB VA 673. 
Table 5.1.: Confirmed and newly identified binaries among our spectroscopic observations or the compiled catalog. Objects with doublelined spectra are marked as SB2. XCF designates cross-correlation profiles with typical binary signatures. RV are radial velocity confirmations, HST astrometry. Approximate spectral types for the primary and secondary reflect mass ratios where available, and are inferred from BCAH models for the age of the Hyades; or are based on individual colors, if not derived in the references.

\begin{tabular}{|c|c|c|c|c|c|}
\hline $2 \mathrm{MASS}$ & Other ID & Primary & Secondary & Notes & Reference \\
\hline \multicolumn{6}{|l|}{ Confirmed binaries: } \\
\hline \multirow[t]{2}{*}{$04480086+1703216$} & LP 416-43, P 95 & M2 & $\sim \mathrm{M} 2$ & $P=10.77 \mathrm{~d}$ & Griffin et al. (1985) \\
\hline & & & & & Wright et al. (2011) \\
\hline \multirow[t]{2}{*}{$04325009+1600210$} & vA 684, vB 91 & K0 & K4 & $P>15$ yr & Griffin et al. (1988) \\
\hline & & & & & Barrado y Navascues \& Stauffer (1996) \\
\hline \multirow[t]{2}{*}{$04322565+1306476$} & vA 677 & K3 & M0 & $\mathrm{RV}$ & Stauffer et al. (1997a) \\
\hline & & & & & Bender \& Simon (2008) \\
\hline \multirow[t]{2}{*}{$04322373+1745026$} & vA 673 & DA2.5 & M3.5 & RV & Lanning \& Pesch (1981) \\
\hline & & & & & Schreiber \& Gänsicke (2003) \\
\hline $04285080+1617204$ & vB 190 & K7 & M3.5 & $\mathrm{RV}$ & Bender \& Simon (2008) \\
\hline $04282878+1741453$ & vA 486 & M1 & M2 -M4 & $\mathrm{RV}$ & Guenther et al. (2005) \\
\hline $04222568+1118205$ & P 33, RHy 173 & K0 & K7 & RV & Bender \& Simon (2008) \\
\hline $04341113+1133285$ & P 79, RHy 334 & K7 & M6.0 & RV & Bender \& Simon (2008) \\
\hline $04145191+1303178$ & vA 68, RHy 116 & $\mathrm{~K} 5 \mathrm{~V}$ & M5V-M6V & $\mathrm{RV}$ & Guenther et al. (2005) \\
\hline $04042701+2024303$ & RHy 49 & $\sim \mathrm{M} 4$ & $\sim \mathrm{M} 4$ & HST & Reid \& Gizis (1997) \\
\hline $04153255+2048274$ & RHy 119 & $\sim \mathrm{M} 5.5$ & $\sim \mathrm{M} 7$ & HST & Reid \& Gizis (1997) \\
\hline $04254701+1732407$ & RHy 221 & 〜M5 & $\sim \mathrm{M} 7$ & $\mathrm{HST}$ & Reid \& Gizis (1997) \\
\hline $04273646+1926454$ & RHy 244 & $\sim \mathrm{M} 4$ & $\sim \mathrm{M} 5$ & HST & Reid \& Gizis (1997) \\
\hline $04352466+1044525$ & RHy 346 & $\sim \mathrm{M} 4$ & $\sim \mathrm{M} 4$ & $\mathrm{HST}$ & Reid \& Gizis (1997) \\
\hline $04270642+1625482$ & RHy 240 & $\sim \mathrm{M} 5$ & $\sim \mathrm{M} 5$ & HST & Gizis \& Reid (1995) \\
\hline $04214955+1929086$ & LP 415-20, Br262 & M6.0 & M9.5 & $P=11.5 \mathrm{yr}$ & Konopacky et al. (2010) \\
\hline $04211974+1202380$ & RHy 158 & M4.5 & & & Reid \& Mahoney (2000) \\
\hline $04403699+1638088$ & RHy 403 & M5.5 & M6.5-L2.5 & RV & Reid \& Mahoney (2000) \\
\hline $04174767+1339422$ & vA 115 & M1 & M5-M9 & RV & Guenther et al. (2005) \\
\hline $04251352+1716056$ & vA 351, RHy217 & $\sim \mathrm{M} 3$ & Ｍ3 & SB3 & Stauffer et al. (1997a) \\
\hline
\end{tabular}


Table 5.1.: Confirmed and newly identified binaries (continued).

\begin{tabular}{llccll}
\hline \hline 2MASS & Other ID & Primary & Secondary & Notes & Reference \\
\hline $04423029+2027115$ & LP 415-345 & $\sim$ M3 & $\sim$ M3 & RV & Stauffer et al. (1997a) \\
$04235070+0912193$ & GH 7-192, LP 535-73 & $\sim$ M3 & $\sim$ M4 & RV & Stauffer et al. (1997a) \\
$04122173+1615033$ & VA45, LP414-117 & $\sim$ M4 & $\sim$ M4 & RV & Stauffer et al. (1997a) \\
$04120760+1737341$ & VA 43, RHy99 & $\sim$ M4 & $\sim$ M4 & RV & Stauffer et al. (1997a) \\
$04235033+1455174$ & VA 288 & $\sim$ M3 & $\sim$ M4 & RV & Stauffer et al. (1997a) \\
$04275919+1845327$ & RHy251, L72 & $\sim$ M3 & $\sim$ M3 & RV & Stauffer et al. (1997a) \\
& & & & & \\
\hline Candidate binaries: & & & & & \\
$04290099+1840254$ & RHy 268 & $\sim$ M4 & later M4 ? & XCF & Stauffer et al. (1987), this work \\
$04360416+1853189$ & RHy 351 & $\sim$ M2 & later M2 ? & XCF & this work \\
$04412966+1313164$ & P 81, vB 316 & K7 & $\sim$ K7 & SB2 & this work \\
$04332699+1302438$ & RHy 322 & $\sim$ M3 & later M3 ? & XCF & this work \\
$04412780+1404340$ & $\ldots$ & $\sim$ M2 & later M2 ? & XCF & this work \\
$04161352+1647481$ & RHy 126 & M5.0 & & HST & Reid \& Gizis (1997) \\
$04395159+1939344$ & RHy 391 & M5.5 & & HST & Reid \& Gizis (1997) \\
$04213775+2018174$ & RHy 162 & M4.5 & & XCF & Reid \& Mahoney (2000) \\
$04214435+2024105$ & RHy 165 & M5.5 & & XCF & Reid \& Mahoney (2000) \\
$04300417+1604079$ & RHy 281 & M5.5 & & RV & Reid \& Mahoney (2000) \\
$04510241+1458167$ & $\ldots$ & $\sim M 0$ & & H $\alpha$ & this work \\
\hline \hline
\end{tabular}


Table 5.2.: Potential photometric binaries.

\begin{tabular}{llrrrr}
\hline $\begin{array}{l}\text { Object } \\
\text { 2MASS J }\end{array}$ & other & $\begin{array}{r}d \\
\mathrm{pc}\end{array}$ & $\begin{array}{r}M_{K_{S}} \\
\mathrm{mag}\end{array}$ & $H-K_{S}$ & Notes \\
\hline \hline $04244805+1552292$ & VA 334 & 50.30 & 4.34 & 0.21 & a) \\
$04251456+1858250$ & LP 415-794 & 51.49 & 5.15 & 0.25 & a) \\
$04290099+1840254$ & vB 278 & 46.21 & 5.37 & 0.26 & a) \\
$04302395+1729591$ & RHy 283 & 56.72 & 5.72 & 0.30 & a) \\
$03545319+1618563$ & GH 7-33 & 44.82 & 5.80 & 0.31 & a) \\
$04262170+1800009$ & RHy 231 & 49.55 & 6.78 & 0.30 & a) \\
$04395159+1939344$ & RHy 391 & 45.00 & 7.17 & 0.33 & c) \\
$04371455+1119268$ & RHy 369 & 50.30 & 7.28 & 0.28 & a) \\
$04180899+1724586$ & RHy 143 & 47.24 & 7.39 & 0.32 & a) \\
$04261903+1703021$ & RHy 230 & 45.00 & 8.64 & 0.37 & c) \\
$04300417+1604079$ & RHy 281 & 45.00 & 8.72 & 0.34 & c) \\
$04385847+1539412$ & RHy 386 & 45.00 & 8.94 & 0.35 & c) \\
$04214435+2024105$ & RHy 165 & 45.00 & 9.24 & 0.36 & c) \\
& & & & & \\
\hline
\end{tabular}

Notes. Distances derived from parallaxes in a) Röser et al. (2011), b) Hanson (1975) or Schwan (1991); c) fixed to 45 pc (cluster center).

We find that of the 21 stars in the observed sample with suspicious $\mathrm{H} \alpha$ line shapes, 12 spectra reveal no further signs of companions based on their clean XCF profiles and a lack of reference work. These stars are likely to exhibit self-absorption in their $\mathrm{H} \alpha$ profiles (Fig. 4.4).

In five of the 21 suspects, we notice a clearly defined asymmetric beaviour in all of their XCFs. One of these targets, 2M 04290099+1840254 (LP 415-121, RHy 268, Pels 71) is among the eight stars found to be rapid rotators by Stauffer et al. (1987). They also suspected a possible binarity, which is supported by the shape anomalies of our XCFs. The separation in radial velocity seems comparable to the spectral resolution of the data, but we do see evidence of line blending from two stars, so that we confirm it as a spectroscopic binary candidate.

Our data reveal four new (spectroscopic) binary candidates, based on the evaluation of line separation and XCFs. 2M 04412780+1404340, 2M 04412966+1313164 (Pels 81, vB 316), 2M 04332699+1302438 (vA 709, RHy 322), 2M 04360416+1853189 (LP 4151582, RHy 351) all show evidence of a companion in their systematically wing-shaped XCFs and have a double-peak structered $\mathrm{H} \alpha$ line core. One spectrum (vB 316) is an obvious SB2, similarly to LP 416-43 with a clearly separated signature of two equal flux contributers, but with $\mathrm{H} \alpha$ in absorption. We treat it in the same fashion by constructing "cloned" template spectra. With a best fit solution of $\Delta R V=34.8 \mathrm{~km} / \mathrm{s}$, following the cloning approach as for LP 416-43 yields a $v \sin i=9.8 \pm 1.5 \mathrm{~km} / \mathrm{s}$ for $\mathrm{vB} 316$.

The asymmetric, but not separated XCFs of the four stars lead us to classify these as potential binaries. We show their $\mathrm{H} \alpha$ profiles in Fig. 5.1 and list them in Table 5.1 as candidates, as additional data at different orbital phases is needed to confirm them. Note that these "candidates" are not plotted as (filled) binaries in any figures, unless explicitly stated. 


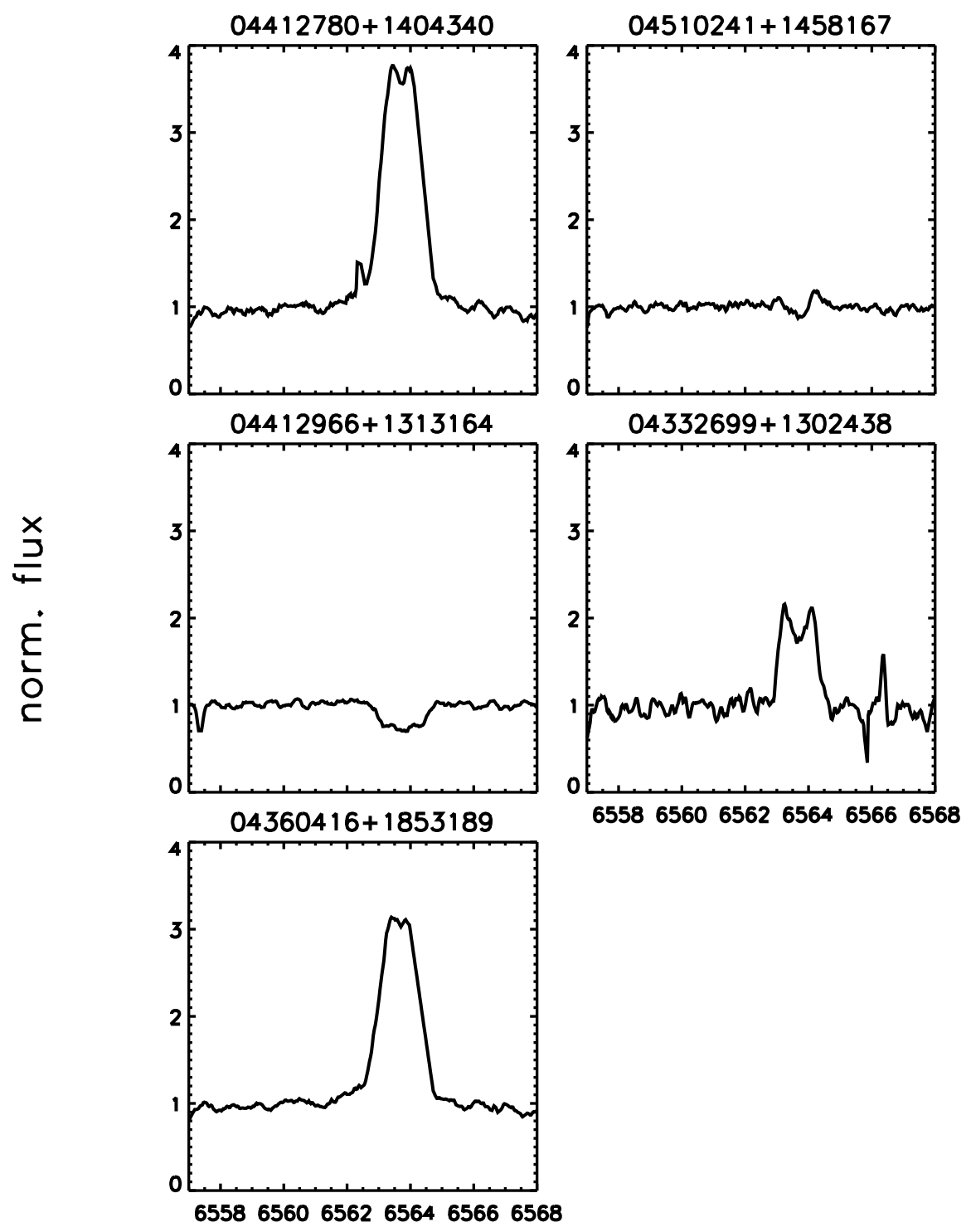

Wavelength [A]

Figure 5.1.: $\mathrm{H} \alpha$ profiles of newly discovered (candidate) binaries. Objects are labelled by their 2MASS identifiers. 


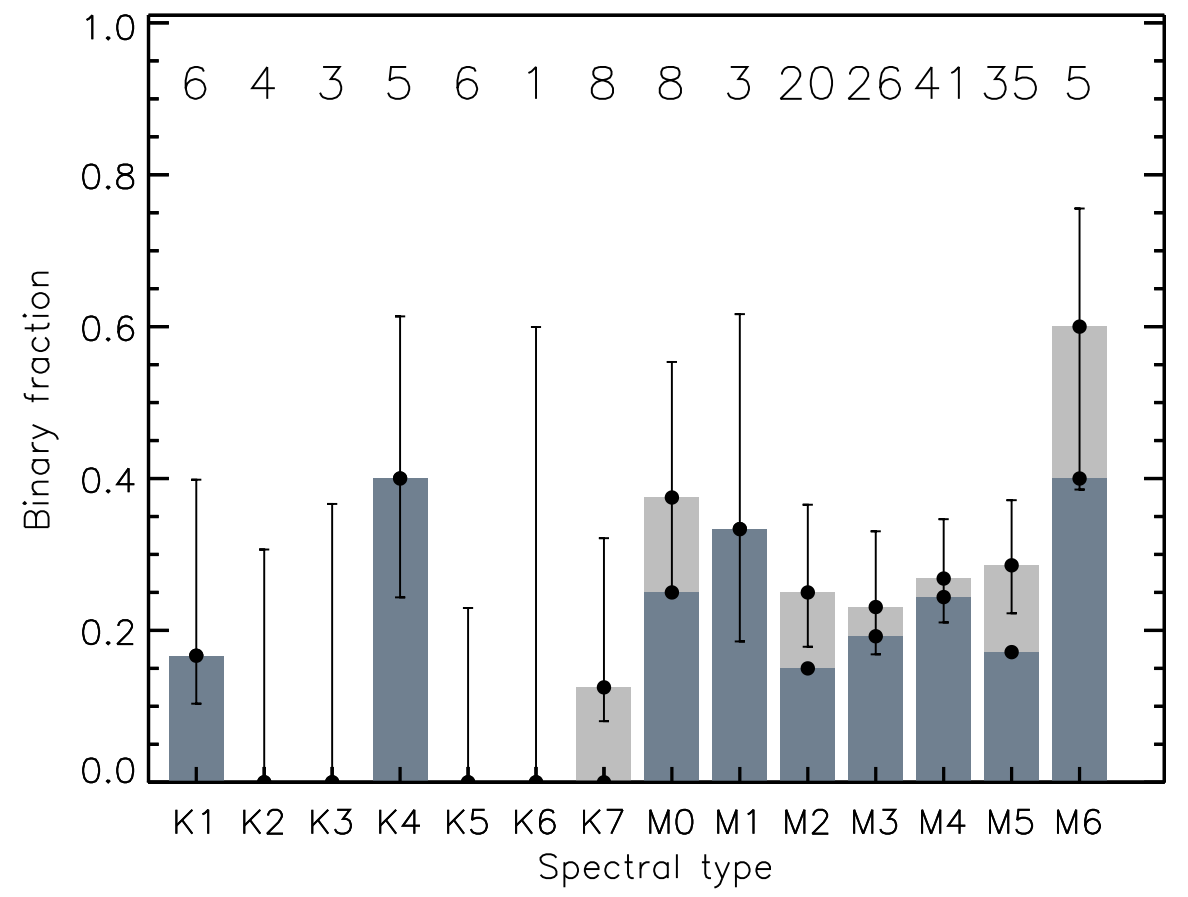

Figure 5.2.: Fraction of binary stars per spectral type among the 171 objects. New candidate binaries (Table 5.1) are taken into account (light bars), else are not considered (dark bars). The overall observed binary fraction is $26 \%$ or $19 \%$, respectively. For readibility, $1 \sigma$ statistical error bars are shown for the light distribution only. Numbers on top of each spectral type indicate the total number of stars in that bin.

Table 5.2 holds 13 objects that we identified in the CMD based on their suspicious photometry (Sec. 4.5). They are brightner and redder than expected for the Hyades MS, and lie above or close to the isochrone for equal-mass binary systems. All of the remaining stars (14) in this regime are confirmed binaries. Individual follow-up observations will show if the 13 suspects are indeed not single stars, which is challenging for these faint stars. At least for three of them further evidence of binarity exists, as they have been noted candidates from HST or RV work (see Table 5.1).

\subsection{Activity}

Magnetic activity is a direct result of the stellar dynamo processes, for which stellar rotation is believed to be a necessary ingredient. Two indicators of activity are measured for the present set of Hyades $\mathrm{K}-\mathrm{M}$ stars: $\mathrm{H} \alpha$ emission is a tracer of chromospheric magnetic activity, originating from magnetic heating. X-ray emission, in contrary, is not tied to individual emission lines, but emanates as broadband flux emission from the thermally heated plasma in the corona. As with other observational proxies of magnetic field generation (calcium emission, photospheric spots, flares), the magnetic field strength is not directly accessible as eg. by means of Zeeman broadening (FeH diagnostic, Reiners \& Basri 2008); however, the direct correspondence between these proxies and the production of magnetic fields is well established. We discuss the results of the activity measurements separately, before relating 


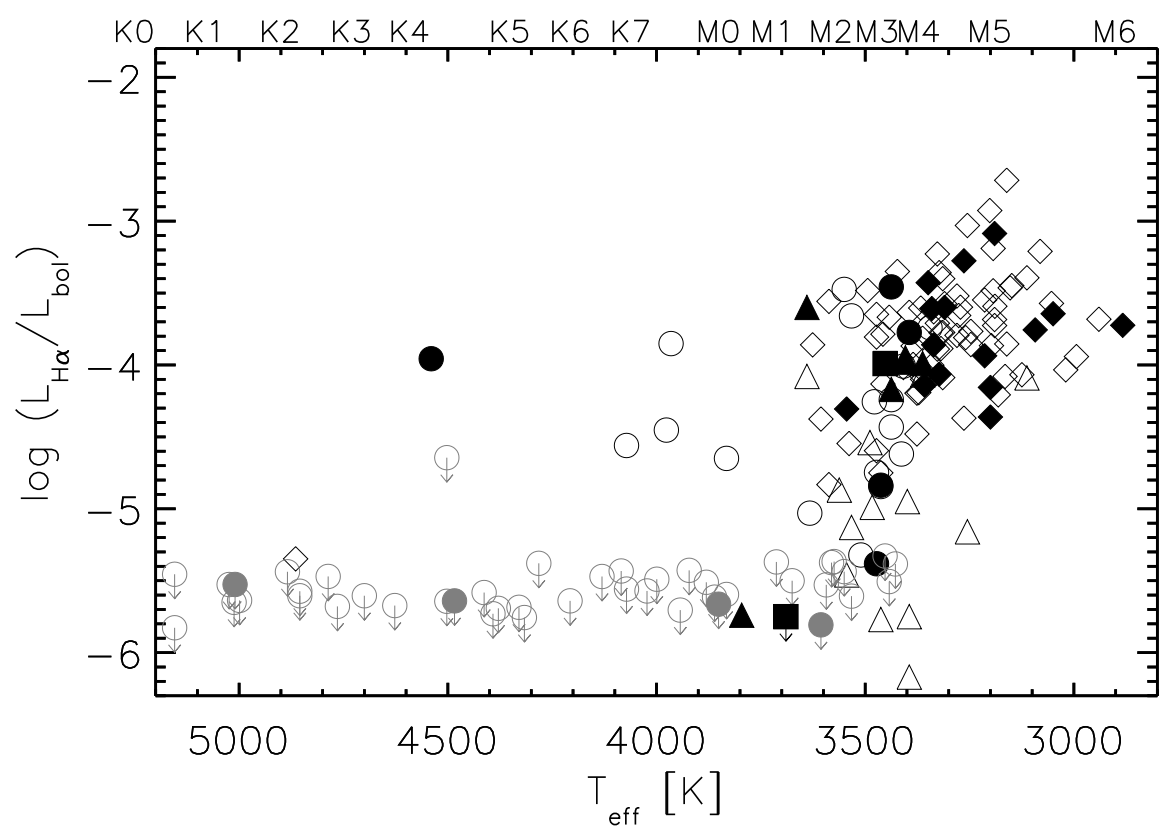

Figure 5.3.: Normalized $\mathrm{H} \alpha$ activity vs. effective temperature (or spectral type) of $\mathrm{K}-\mathrm{M}$ dwarfs in the Hyades. Open symbols denote single stars, filled symbols binary stars. Non-detections (upper limits, with arrows) are greyed out. Symbol shapes represent the origin in the combined catalog, with objects as squares (Radick et al. 1987; Seifahrt et al. 2009), triangles (Stauffer et al. 1987, 1997a), diamonds (Reid \& Mahoney 2000, and references therein), and circles (this work). Chromospheric magnetic activity ( $\mathrm{H} \alpha$ emission) is marginally observed in the $\mathrm{K}$ stars (inactive) until it becomes prevalent among the M-type stars. By M5, no object is seen as inactive. A limiting saturation level of $\log \left(L_{\mathrm{H} \alpha} / L_{\mathrm{bol}}\right) \approx-3.5$ is reached at around the same region where activity sets in.

the two proxies to each other. Rotation will be discussed after, and the three aspects brought into context.

\subsubsection{Chromospheric activity}

Measurements of $\mathrm{H} \alpha$ activity for the entire sample are presented in Fig. 5.3. The general distribution follows the expected trend observed in (older) field stars. K stars are observed as inactive, with average measured levels of $\log \left(L_{\mathrm{H} \alpha} / L_{\mathrm{bol}}\right) \approx-5.5$ as upper limits. We distinguish between single stars (plotted as open symbols in Fig. 5.3) and established binaries (filled), and both types show the same trend (a few notable exceptions exist). A dramatic increase in activity levels is seen among the early $\mathrm{M}$ stars. Beginning at $T_{\text {eff }} \approx 3700 \mathrm{~K}, \mathrm{H} \alpha$ activity levels rise by two orders of magnitude, and then continue on this level on average towards cooler temperatures. A transition region exits where both inactive and active stars coexist $(3700-3400 \mathrm{~K})$, corresponding to spectral types M1-M4. For later types, no inactive stars are observed, and $\mathrm{H} \alpha$ maintains a high level. This behaviour is seen for single and binary stars alike.

A few active objects are present among the hotter stars that do not follow the general inactivity floor, but that show considerably enhanced $\mathrm{H} \alpha$. These are four presumably single 
stars and one binary that deserve further mention (one further star, 2M 4235440+1403075 (vA 294), is an upper limit due to a low S/N spectrum). 2M 04322565+1306476 (vA 677) displays unusually high activity compared with other stars of similar spectral type in Fig. 5.3. It consists of a K3/M0 pair with a short orbital period of $1.48 \mathrm{~d}$, and a separation of $d \sin i \approx$ $0.02 \mathrm{AU}$ has been reported from direct imaging of this close system (Bender \& Simon 2008). This may explain its activity, as in such a system, interactions or induced $\mathrm{H} \alpha$ are likely similar to what is seen in post common envelope binaries. We discuss this further in Sec. 6. Our value in Fig. 5.3 for vA 677 takes into account $\mathrm{H} \alpha$ emission components from both stars, but even when we perform a fractional analysis ( $64 \%$ is contributed by the redder component), the resulting $\log \left(L_{\mathrm{H} \alpha} / L_{\mathrm{bol}}\right)=-4.41$ and -4.15 , respectively, are still on a significantly enhanced level. Given the rapid rotation of the (compound) system (Table A.1), we can compare this to the rotational velocity the star would show if the system were tidally locked, based on the orbital period. This yields $v_{\text {rot }} \approx 20 \ldots 27 \mathrm{~km} / \mathrm{s}$ as an estimate-depending on the assumed radius - which is very similar to what we actually measure for the system $(v \sin i=23.4 \pm 1.2 \mathrm{~km} / \mathrm{s})$. Thus, the reason for vA 677 showing enhanced activity (and rotation) that sticks out of the population of stars in the K-type range is likely a tidally locked system. As a result, this object cannot be compared directly to the single counterparts.

The remaining four active stars of type K7-M0 in Fig. 5.3 are somewhat puzzling. They appear in contrast to the very sharp and abrupt onset of $\mathrm{H} \alpha$ at slightly cooler temperatures, which is remarkable on its own. All four objects have precise distances measured from parallaxes, so we can rule out a largely misclassified luminosity due to a distance effect. If they were background stars, we would underestimate their bolometric luminosities, which in turn would make them appear more active in Fig. 5.3. We do not see any indications of binarity either, that could justify a misclassification of spectral type for these objects. Inspecting the corresponding spectra reveals very prominent $\mathrm{H} \alpha$ emission lines with strong self-absorption in the line cores, the $\mathrm{H} \alpha$ activity levels thus also appear sound. We see this as a hint that the stars in question could be randomly caught during active flaring events with strong activity outbursts, fostered temporarily due to variable magnetic activity. Or they could reveal a separate population of active stars in the otherwise inactive regime. If these stars were not part of the natural distribution of growing chromospheric activity as a function of spectral type (but instead represented some other underlying mechanism unknown to us), the step function-like changeover from quiescent to enhanced activity levels (coined "H $\alpha$ limit") would appear offset. In that case, the $\mathrm{H} \alpha$ limit would appear as an abrupt surge between spectral types M1/M2, at the same time mapping the much increased number density of stars per spectral type bin at precisely the same position. While our catalog contains only eleven M0-M1 stars, this number jumps to 46 in the M2-M3 bins. A selection effect is therefore the more probable reason for the sharp increase at M1/M2, rather than a general paucity of active stars of type M0/M1. We argue that with more data points in the M0/M1 bins, the sharp increase in $\mathrm{H} \alpha$ levels smears out into a smoother transition. The four active stars earlier than M1-type thus are extremely unlikely to belong to a separate population, but are evidence of a somewhat smooth transition in $\mathrm{H} \alpha$. First, even if these objects are flare stars in Fig. 5.3, their quiescent state would most probably still exhibit $\mathrm{H} \alpha$ activity above the level of the other stars at that spectral type. Second, all four of these stars also show increased $\mathrm{X}$-ray emission (Sec. 5.2.2), which adds support for an enhanced quiescent activity level. Note that the X-ray and the $\mathrm{H} \alpha$ data have quite different observing epochs, so flaring stars are not necessarily seen in both indicators. Third, $78 \%$ of the objects in this particular range of spectral types, which show either increased chromospheric or increased coronal activity, also exhibit enhanced rotation $(6 \gtrsim v \sin i \lesssim 10 \mathrm{~km} / \mathrm{s})$. As we will see when discussing the 


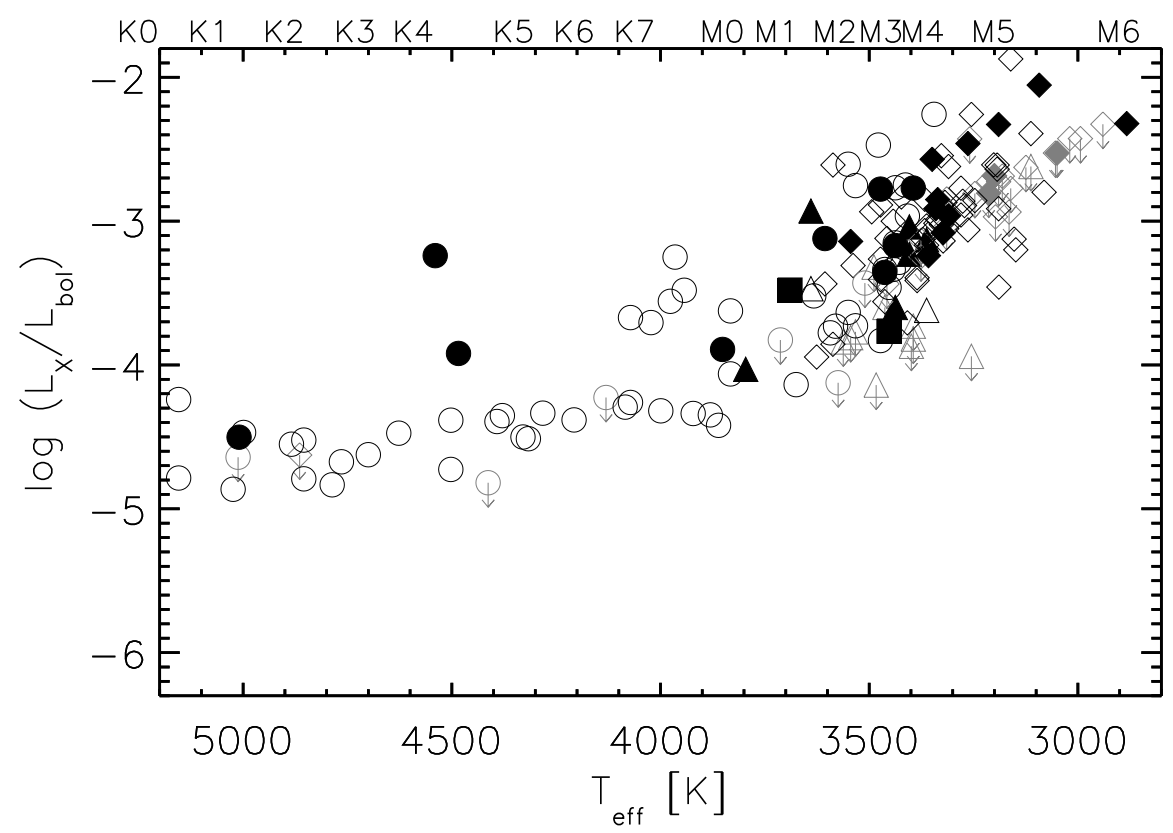

Figure 5.4.: Normalized X-ray emission luminosity vs. effective temperature (or spectral type) for Hyades $\mathrm{K}-\mathrm{M}$ dwarfs. Open symbols denote single stars, filled symbols binary stars. Non-detections (upper limits, with arrows) are greyed out. Symbol shape as in Fig. 5.3. Coronal magnetic activity (X-ray emission) strongly increases for spectral types M.

rotation-activity relation in Sec. 5.3.3, this advocates against a sharp $\mathrm{H} \alpha$ limit at spectral type M1/M2, but instead promotes the existence of elevated activity levels in the K7-M1 spectral type range. The data thus indicates an $\mathrm{H} \alpha$ limit in precisely this range, located between type K7 and M1, albeit a higher count of observations in this range would further substantiate its locus.

Hawley et al. (1999) have investigated the $\mathrm{H} \alpha$ limit and determined its locus in a few open clusters of different ages. For the Hyades, they report the change from inactivity to prevalent activity to occur around spectral type M2, albeit with substantial uncertainty (around M2 when based on $V-I$ data, within K4-M2 when based on bolometric magnitudes; see their Figs. 5 and $6 \mathrm{~b}$ ). Concurrently, the data in our Fig. 5.3 agrees with an $\mathrm{H} \alpha$ limit no later than at type M2 well, and constrains the $\mathrm{H} \alpha$ limit towards one to three sub-types earlier as argued above.

\subsubsection{Coronal activity}

Similar to Fig. 5.3, we show in Fig. 5.4 the distribution of normalized X-ray emission luminosity as a function of effective temperature (spectral type). X-ray emission in main sequence stars as a tracer of coronal magnetic activity is well known from the Sun. Diffuse continuum $\mathrm{X}$-ray emission from cool stars is attributed to stellar coronae as a result of magnetically driven, hot plasma (Güdel 2004). In particular, mass ejections and accelerated wind particles can trigger strong X-ray emission.

For the earlier type stars in our catalog, coronal activity stays at a relatively quiescient level until beginning with spectral type $\mathrm{K} 7, L_{X} / L_{\mathrm{bol}}$ quickly gains strength towards cooler 


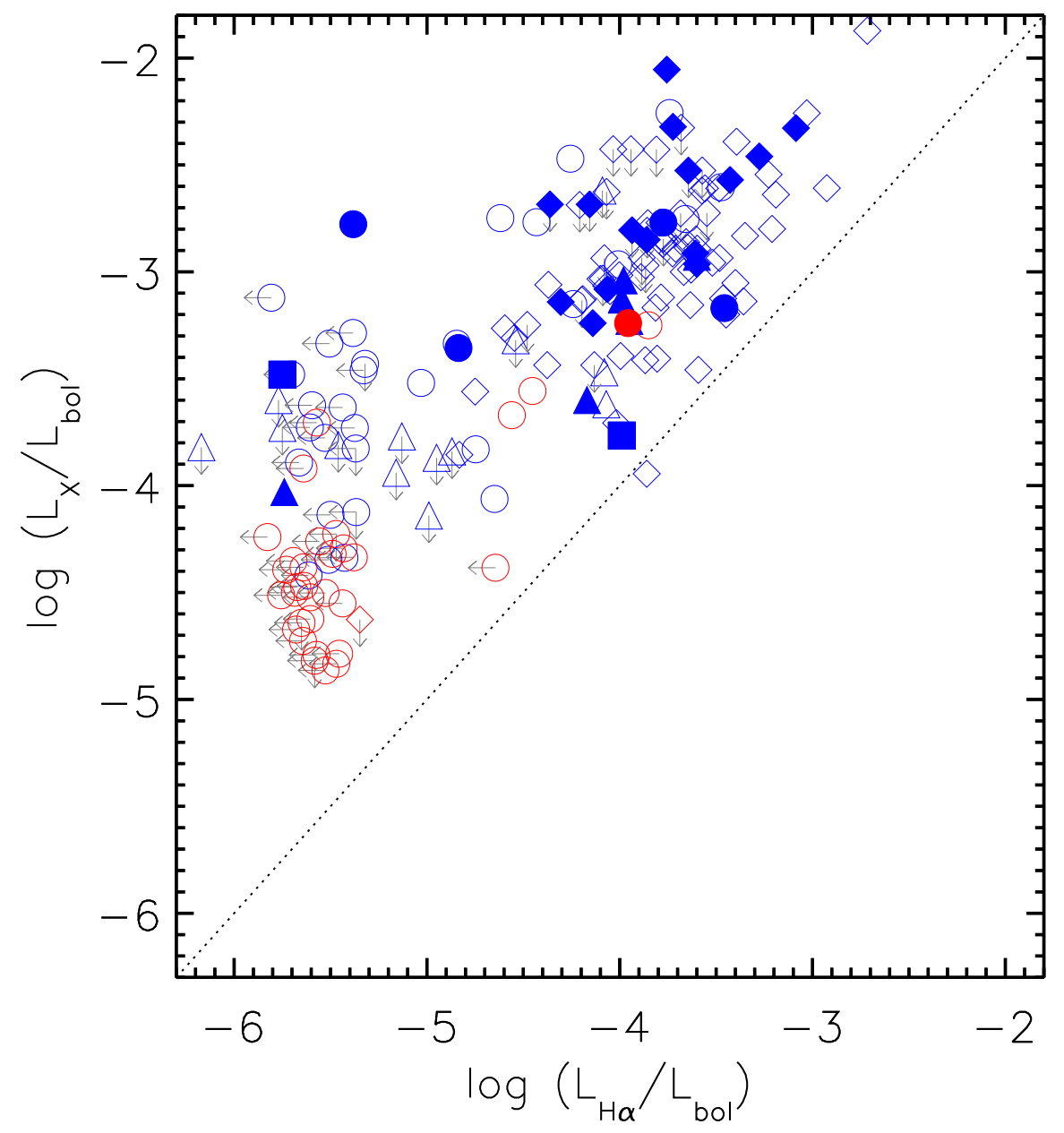

Figure 5.5.: Chromospheric versus coronal activity (normalized by the bolometric luminosity) for spectral types $\mathrm{K}-\mathrm{M}$ in the Hyades. K-type stars are plotted in red, M-type stars in blue. Filled symbols denote binary stars. Upper limits in $L_{\mathrm{H} \alpha}$ (left arrows) and $L_{X}$ (down arrows). Symbols as in Fig. 5.3. Generally, strong $\mathrm{H} \alpha$ equates to strong X-ray emission.

temperatures. By spectral type M4, no object is seen at the previously quiescient level anymore. As expected, this happens at the same mass as for $\mathrm{H} \alpha$. It is noteworthy that the $L_{X}$ activity distribution is smoother than for $\mathrm{H} \alpha$ with a less abrupt onset. The envelope of maximum X-ray emission (saturation) is reached at spectral type M4 and stays constant until the latest stars in the sample, albeit the level of saturation is an order of magnitude higher than in the chromospheric counterpart.

There is no obvious difference between the single stars and the binaries in Fig. 5.4. However, a few stars need to be discussed in detail as they stand out. Two binary stars among the $\mathrm{K}$ dwarfs display obvious high levels of X-ray activity. Firstly, the more active of the two is again vA 677 mentioned above. The strong X-ray emission in this close binary further supports a tidally locked system with fast rotators, responsible for generating the $\mathrm{H} \alpha$ and $L_{X}$ activity levels. The second object (2M 04145191+1303178, vA 68) is found to be a K5/M5 binary from direct imaging (Guenther et al. 2005). The observed separation $\left(0.62^{\prime \prime}\right)$ translates into an upper limit of $29 \mathrm{AU} \times \sin i$, so that an interacting close binary is unlikely (H $\alpha$ emission is 
not detected). As pointed out earlier, the X-ray data are not simultanously recorded with the spectroscopic observations. However, the mid M-dwarf companion on its own can be expected to exhibit significant X-ray emission following the population in Fig. 5.4, in which case our observed value is underestimated (due to the much lower respective bolometric luminosity of the M-dwarf alone), as our measurement of $L_{X} / L_{\mathrm{bol}}$ reflects the integrated $L_{\mathrm{bol}}$ of the binary. Discarding the potential flux contribution from the higher-mass star, the datum for vA 68 in $\mathrm{X}$-rays would then be another measurement of an isolated M5 star with $L_{X} / L_{\mathrm{bol}}=-3.27$ (corrected for $L_{\mathrm{bol}}$ ), placing it consistently on the lower $L_{X}$ envelope.

A number of data points in Fig. 5.4 are clustered in the K7-M0 range at increased X-ray activity, similar to what is seen in $\mathrm{H} \alpha$. Interestingly enough, this active subset is only seen in the very same narrow regime as it is observed in $\mathrm{H} \alpha$ (Fig. 5.4). These distinct objects are not necessarily active in both $L_{X}$ and $\mathrm{H} \alpha$, in fact, some of them are either active in the one or the other activity indicator. We note again that observations of the two proxies were not at all simultanous, but offset by $\approx 15$ years. However, all the stars of this subset that are active in $\mathrm{H} \alpha$ are also very active in X-rays, notably the aforementioned vA 750, 2M 05110971+1548574, and LP 474-1171. These stars are intrinsically very active and exceptions in this otherwise rather quiescient regime, and lend support to the idea that the population of active stars in the Hyades extends into the vicinity of spectral type $\mathrm{K} 7$ (and thus with an $\mathrm{H} \alpha$ - and X-ray limit shifted to the latest $\mathrm{K}$ stars, at earliest). The remaining of these stars with either high $\mathrm{X}$-ray or $\mathrm{H} \alpha$ levels can be attributed to temporal flaring activity, although we do not observe obvious flare stars in other spectral type regions.

As already noted in Sec. 4.3, we only detect X-ray emission upper limits in a number of stars of the latest spectral types. This is not surprising, given a) the sensitivity of the ROSAT observations considered here, b) the intrinsic faintness of these late M-dwarfs, and c) the distance to the Hyades cluster. It is thus important to realize that the distribution of detected $\log L_{X} / L_{\mathrm{bol}}$ at the low-mass end of our catalog is biased towards higher activity levels. In particular, the paucity of very low $L_{X}$ detections bears an imprint of the sample selection (ie. target faintness). This does not mean that the true distribution lacks inactive stars - we just do not detect them in our sample. Although the limitations are the same in the field, at closer distances the $\log L_{X} / L_{\text {bol }}$ distribution for field stars is also populated in the inactive regime, where nearby stars of type M5 are observed down to $\log L_{X} / L_{\text {bol }} \approx-6$ (Voges et al. 1999). In Fig. 5.4, the observed lower envelope in $\log L_{X} / L_{\text {bol }}$ approaches levels of -2.5 to -3 for the latest Hyades types. However, a handful of stars later than M5 (plus a few more upper limits) exhibit $\log L_{X} / L_{\text {bol }}$ as strong as -1.9 , probably also caught during peak levels of variable activity.

Our effort to pair the optical proxy of activity, $\mathrm{H} \alpha$, with observations of X-ray emission from the corona is mapped in Fig. 5.5 for the sample stars. From this, the interplay between $\mathrm{H} \alpha$ and X-rays can be divided into four groups. Inactive stars show neither of the two, whereas active stars do exhibit enhanced levels in both proxies. No object shows high $\mathrm{H} \alpha$ and no X-ray emission - which is expected: while $\mathrm{H} \alpha$ emission needs to overcome the stellar continuum emission to be detected as such, no such continuum is irradiated in X-rays, and $\mathrm{X}$-ray emission is only triggered in the presence of magnetic activity. Conversely, a group of Hyades stars with low $\mathrm{H} \alpha$ emission but enhanced X-ray emission exists (detections, not upper limits), and this is mostly true for M-stars (plotted in blue in Fig. 5.5), but also for a few K-stars (in red).

Within the latter group, 2M04341113+1133285 (vB 294) takes an extreme position of $\log L_{X} / L_{\mathrm{bol}}=-2.78$. Similarly to vA 68 , this binary is comprised of a K7/M6 system (Bender \& Simon 2008). If the X-ray detection is solely due to the later type companion, 


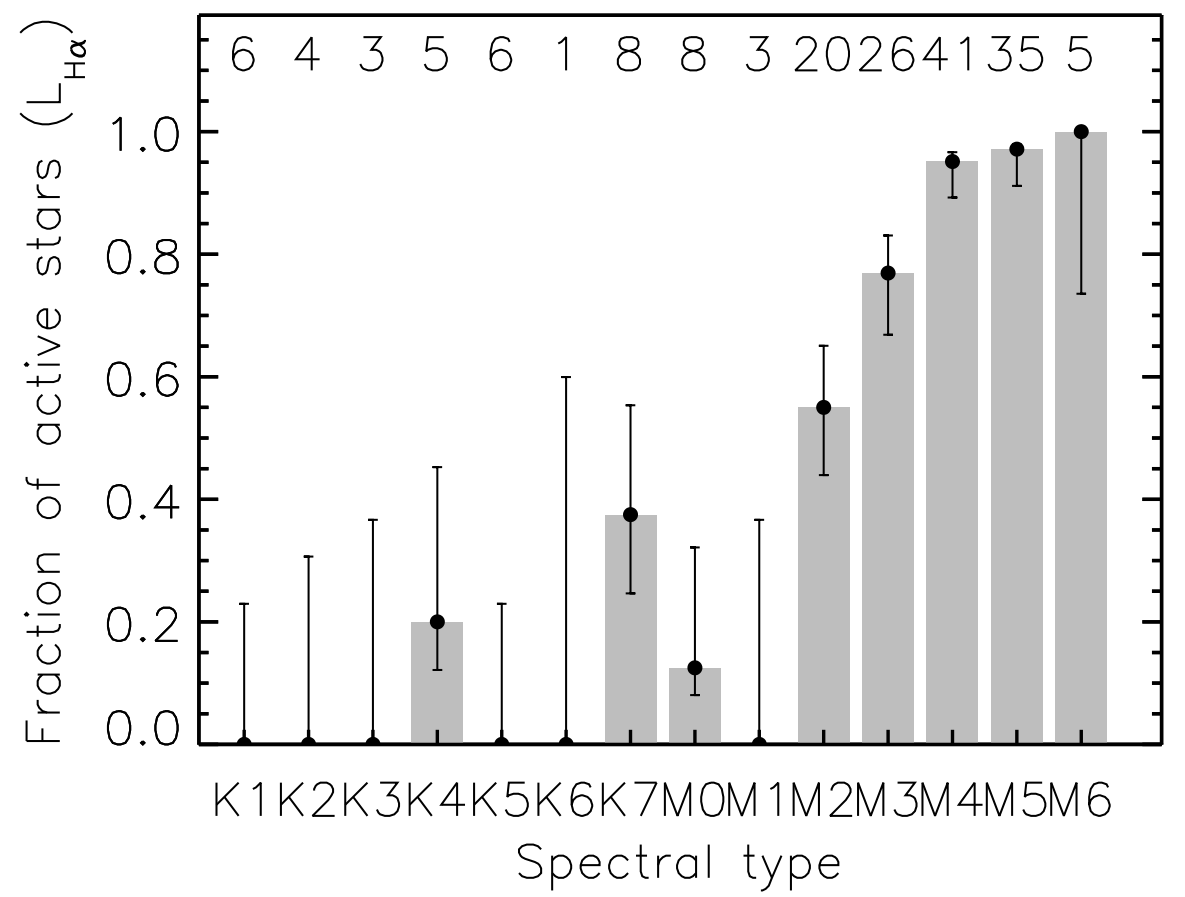

Figure 5.6.: Fraction of chromospherically active stars (ie. with $\mathrm{H} \alpha$ emission) per spectral type. Numbers indicate the total number of stars per spectral type bin, error bars refer to $1 \sigma$ uncertainties.

it's lower luminosity places the star down to $\log L_{X} / L_{\text {bol }}=-3.1$ in Fig. 5.5, further towards the other objects in the same group (still at a considerably high level though, yet restoring consistency).

The general picture of activity in the Hyades $\mathrm{K}-\mathrm{M}$ stars follows the rule that strong $\mathrm{H} \alpha$ equates to strong X-ray emission. This also seems true for binary stars, however, it is surprising that we do not see any binaries among the non-active group of $\operatorname{stars}^{2}$ in Fig. 5.5. In effect, the chromospheric-coronal emission relation at $625 \mathrm{Myr}$ in the Hyades appears no different than in field stars, where it is well characterized (eg. Hempelmann et al. 1995; Pizzolato et al. 2003; Reiners et al. 2009).

\subsubsection{Activity fraction}

From the measurements of $\mathrm{H} \alpha$ emission it is evident that strong chromospheric magnetic activity is found in Hyades $\mathrm{M}$ stars. We also find $\mathrm{H} \alpha$ activity in $12 \%$ of the K-type stars (compared to $80 \%$ in the M-stars). Fig. 5.6 shows the distribution of "active" stars, i.e. those with detected $\mathrm{H} \alpha$ in emission, as a function of spectral type. A cut-off threshold of $\log L_{\mathrm{H} \alpha} / L_{\mathrm{bol}}=-5$ is applied in Fig. 5.6 to mark inactive objects. There is a clear jump in the fraction of active stars observed. From the $80 \%$ active stars of all $\mathrm{M}$ stars in the catalog, by spectral type M2 more than $55 \%$ are in emission, further growing to $95 \%$ by M4. On the

\footnotetext{
${ }^{2}$ We count $2 \mathrm{M} 04174767+1339422(\mathrm{vA} 115)$, on the verge of being inactive $\left(\log L_{X} / L_{\mathrm{bol}}=-4.06\right)$, to the group of stars with marginal $\mathrm{H} \alpha$ emission and slightly increased X-ray emission. This star is found to have a low-mass companion of spectral type late M (Guenther et al. 2005), so that possibly again the lower mass companion may be responsible for the bulk X-ray emission, in which case our derived $\log L_{X} / L_{\mathrm{bol}}$ is underestimated although the origin of the $\mathrm{H} \alpha$ emission is unclear then.
} 


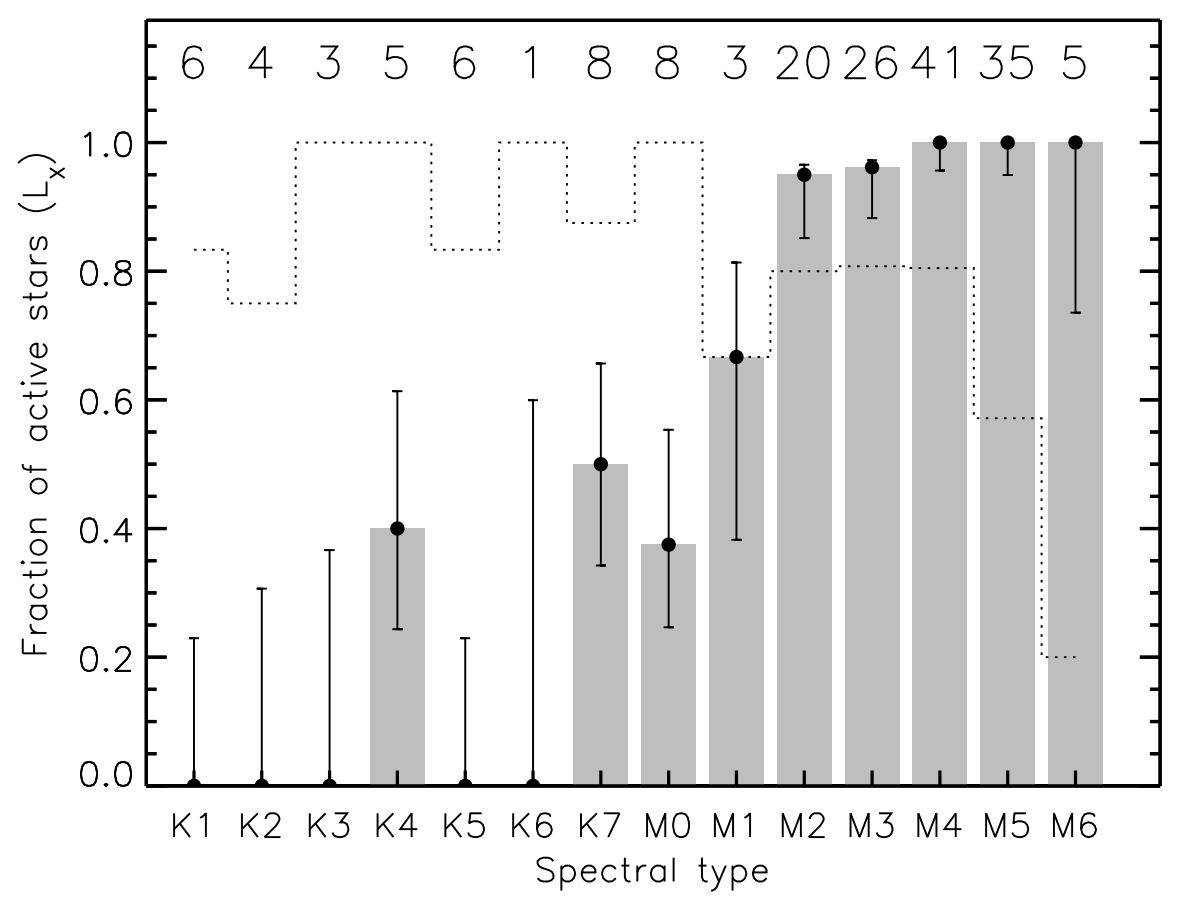

Figure 5.7.: Fraction of coronally active stars per spectral type. Active stars have detected coronal X-ray emission $\log L_{X} / L_{\mathrm{bol}}>-4$. Numbers indicate the total number of stars per spectral type bin, error bars refer to $1 \sigma$ uncertainties. Note that the X-ray detection limit is tightly correlated with spectral type (ie. object faintness), indicated by the dotted outline. At M6, only the most $L_{X}$ luminous Hyades are detected.

contrary, among the K-types, basically no activity is observed until the very latest K-types (four objects do show $\mathrm{H} \alpha$ in emission, see below).

Given the low number of stars in the earlier spectral type bins, particularly K6 and M1, the distribution appears somewhat unsmooth. However, active stars do exist in this regime as pointed out in Sec. 5.2.1. Hints for a turnover in the distribution have been noted already by Stauffer et al. (1991, 1994): the frequency of active stars quickly rises towards later spectral types, and this transition region is confined to a range of a few spectral types. In field stars, this region has been demonstrated to be M3-M4 (eg. West et al. 2004, 2008; Reiners et al. 2012). As other authors have suspected before (Hawley et al. 1999), we notice a shift of this transitional region towards earlier spectral types, i.e. between K7 and M2, at latest, for the Hyades open cluster stars.

From the X-ray perspective of activity, the picture is substantially simpler. Fig. 5.7 demonstrates, that coronal X-ray emission is detected in a high fraction of $80 \%$, on average, of all spectral types covered, and essentially the distribution is very similar to $\mathrm{H} \alpha$. Note that the detection of $L_{X}$ towards later types is hampered by their intrinsic faintness, ie. the detection limit. This results in a bias in Fig. 5.7 and explains the drop-off among the latest types (indicated by the dotted outline). By spectral type M6, the sensitivity of the observations (ROSAT, though with different levels of deepness depending on mode; cf. Table A.1) is on par with the level of X-ray emission from the most active stars (Fig. 5.4), so that the low detection rate is of no surprise. 

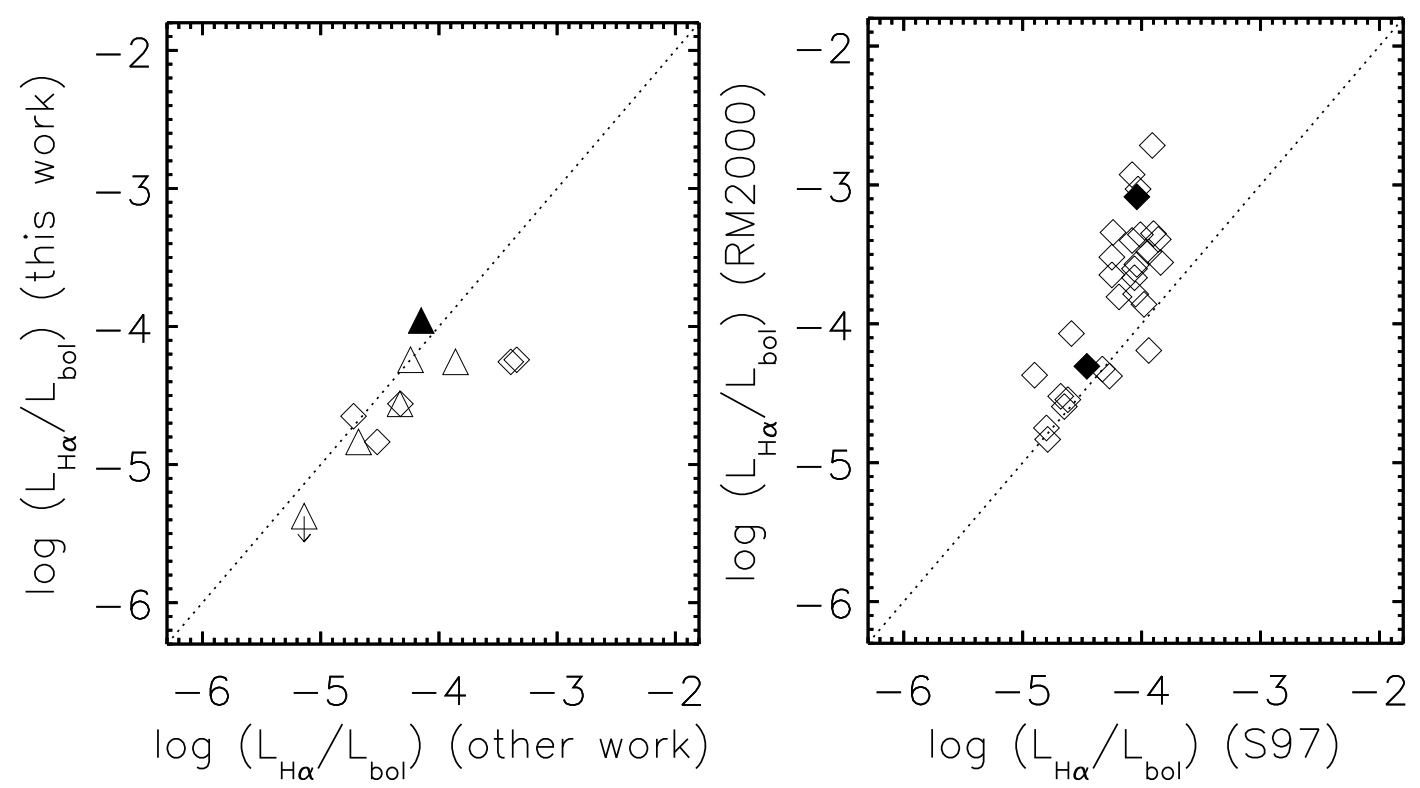

Figure 5.8.: Comparison of detected activity strengths with previous measurements. Left panel: chromospheric $\mathrm{H} \alpha$ emission luminosity for stars in our sample in common with Stauffer et al. (1991, 1997a, triangles) and Reid \& Mahoney (2000, diamonds). Right panel: comparison of $\mathrm{H} \alpha$ emission for stars included in both S91/S97 and RM2000. Filled symbols indicate confirmed binary stars, arrows are upper limits, respectively.

In Fig. 5.7, the ratio of active objects with $\log L_{X} / L_{\text {bol }}>-4$ resembles the behaviour seen in chromospheric activity (Fig. 5.6), again with a clear increase in X-ray emission strength at spectral type K7/M0 (for the K4 bin, see above). Where inactivity seemed prevalent in $\mathrm{H} \alpha$ in the few objects of spectral type M0/M1, X-ray activity is observed in the majority of them, supporting a homogenous onset in activity in the early M-stars. Fig. 5.7 further underlines the approximate correspondence between between coronal and chromospheric activity.

\section{Comparison to previous results}

A few stars in the observed sample are in common with previous measurements from the series of papers by Stauffer et al. This provides a means of comparison with our method of measuring the $\mathrm{H} \alpha$ emission strength relative to the bolometric luminosity of the star. While we base $L_{\mathrm{bol}}$ on stellar models, other authors have used simpler empirical relations between photometric magnitude and colour to derive the bolometric luminosity. A strong emission in e.g. H $\alpha$ can change the flux in optical filter bands significantly - mostly in $R$-but secondary activity proxies like calcium, other balmer lines, and sometimes helium emission can have flux contributions in various wavelength ranges, rendering precise photometry difficult. A comparison of the two approaches is plotted in Fig. 5.8 (left). Our values generally seem to be in good correspondence with the ones by Stauffer et al, with a slight offset of approximately -0.25 for most stars to lower luminosities that supports the aforementioned. With a small number of stars in comparison, we cannot exclude a significant bias from stellar temporal variability, so that larger discrepancies are likely caused by random, enhanced activity levels and flares at the time of observation. We attribute the two objects with the strongest previous 


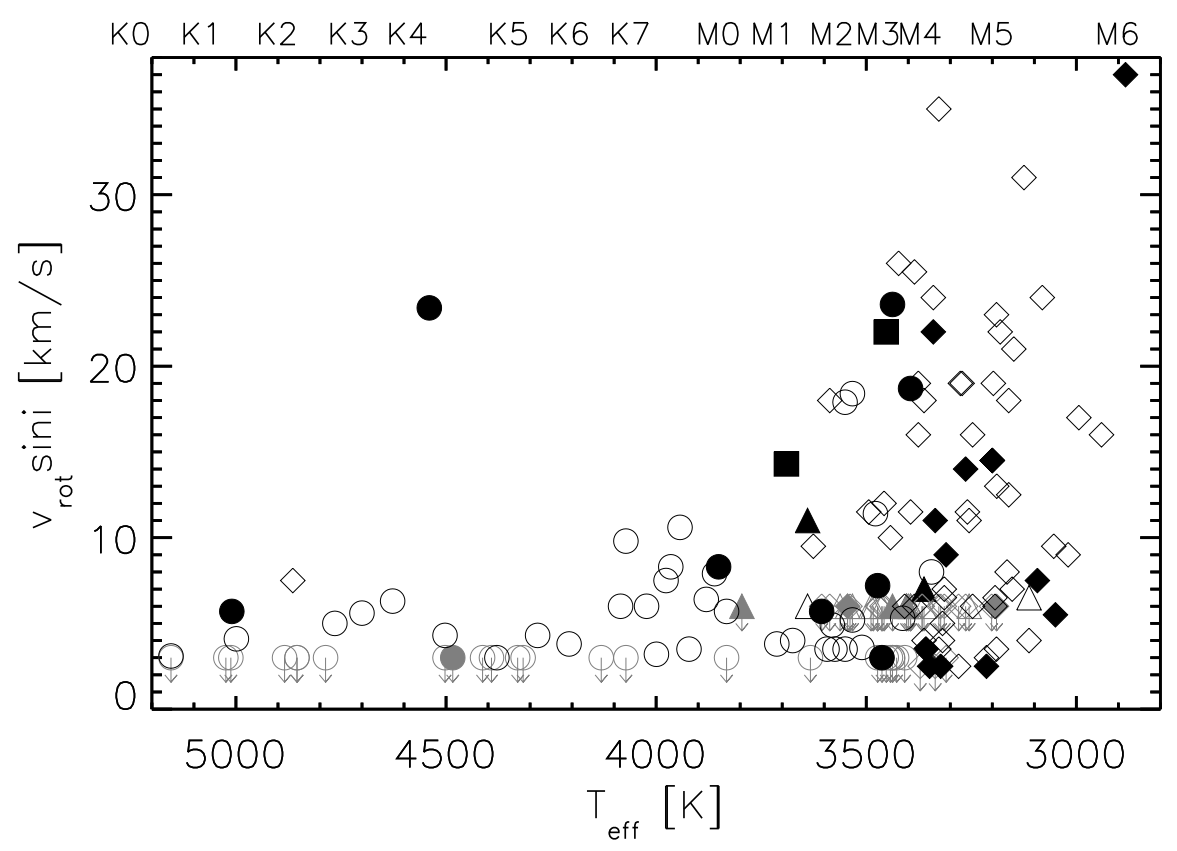

Figure 5.9.: Measured rotational velocities $v_{r o t} \sin i$ as a function of temperature (with corresponding spectral types) for Hyades $\mathrm{K}-\mathrm{M}$ type stars. Non-detections $\left(v_{\text {rot }} \sin i\right.$ below the detection limit) are marked as grey arrowed symbols. Single stars are indicated by open, binary stars by filled symbols. Different shapes as in Fig. 5.3. The isolated rapid binary at $T_{\text {eff }}=4550 \mathrm{~K}$ is $2 \mathrm{M} 04322565+1306476$ (see text), the most rapid rotator is $2 \mathrm{M} 04214955+1929086$ at M6.

measurements, now seen less active by approximately an order of magnitude, to this effect.

On the contrary, we find a large difference in Fig. 5.8 (right) between data from Reid \& Mahoney (2000) in common with Stauffer et al. (1997a, 1991), where the former measure systematically stronger $\mathrm{H} \alpha$ emission than the latter. Two regimes exist, where stars in the inactive part $\left(\log L_{\mathrm{H} \alpha} / L_{\mathrm{bol}} \lesssim-4.3\right)$ have consistent values within a factor of upto three. In the more active part $\left(\log L_{\mathrm{H} \alpha} / L_{\mathrm{bol}} \gtrsim-4.3\right)$, differences amount to upto $\times 16$, with an average enhancement of $\times 3$. Given the number of objects affected, this is hardly explained by activity variability alone. The Stauffer et al. data seems to saturate around $\log L_{\mathrm{H} \alpha} / L_{\mathrm{bol}}=-4$, which points to discrepancies in $L_{\mathrm{bol}}$ arising from their colour calibration (and possibly differences in obtaining $L_{\mathrm{H} \alpha}$ ). We take this as further support for our homogenous methodology.

\subsection{Rotational velocities}

Stellar rotation is the major constituent of spectral line broadening in main sequence stars. The effect is independent of wavelength and results from the integrated Doppler-shifts of light from the advancing and receding parts of the rotating stellar disc. As such, rotational broadening is a symmetric effect, and imposed equally sided on all spectral (photospheric) lines. While ealier type stars on the main sequence can reach several hundreds of $\mathrm{km} / \mathrm{s}$, the rotational velocity of low-mass stars is of order $50 \mathrm{~km} / \mathrm{s}$ and below, and thus requires a high spectral resolving power to be disentangled. The signature of a similar type companion star may mimick the presence of rotational broadening (cf. Sec. 4.4), if the separation in RV is 
small enough, ie. if the orbital phase is close to zero; or the orbital period of the companion is long. A much lower mass companion is also difficult to detect, as the spectral contribution of the lower luminosity object may not be detectable against the brighter one, and the line separation in RV is naturally further reduced. This means that any measurement of increased rotational velocity is, in principle, prone to contamination by unseen companions, and this needs to be kept in mind.

We show the measured projected rotational velocities $v \sin i$ in Fig. 5.9 for all spectral types in the sample of Hyades stars. The distribution of $v \sin i$ is controlled by two regions: K-type stars (until K7) are observed with significant rotation rates (on the order of $5 \mathrm{~km} / \mathrm{s}$ ), whereas this behaviour dramatically changes in the M-dwarfs, where rapid rotation seems to be common on the $10-30 \mathrm{~km} / \mathrm{s}$ level. Fig. 5.9 displays a few interesting aspects. First, there does not seem to be a significant difference between single and binary stars. We find that the (detected) binaries populate all parts from non-rotators (below the detection limit) to the highest rotation as do the single stars, across the entire range of spectral types. The strikingly rapid K-type object $(v \sin i=23.4 \mathrm{~km} / \mathrm{s})$ is an obvious exception. This binary (vA 677) has already been discussed in Sec. 5.2.1, and we only note here again that it is likely a tidally locked system. Evidence for this scenario is drawn from its close orbital separation in conjunction with its exceptionally high activity and rotation for its kind. vA 677 therefore should be regarded differently than the single stars and the many other binaries in the sample that apparently behave no different than the single stars.

Secondly, our sample shows that for stars cooler than $3200 \mathrm{~K}$ (later than M5), we do not find any non-rotators, with the lower envelope of $v \sin i$ rising towards the coolest objects in the sample.

\subsubsection{Fraction of rotators}

Our analysis shows rotation in a number of stars at spectral types mid-K. We also find evidence for rotational velocities among the K7-M1 stars that are clearly above the detection limit, with $v \sin i$ up to about $10 \mathrm{~km} / \mathrm{s}$. Even if we drop the only two detected binaries among them, the evidence is striking. Fig. 5.10 illustrates this behaviour and shows the fraction of stars with detected rotation. Around $40 \%$ of the K7 to M1 stars have rotational velocities larger than $6 \mathrm{~km} / \mathrm{s}$, in contrast to earlier spectral types, and also to the adjacent cooler types. We note that the low number of stars in the K6 and M1 bins (see the histogram of rotation in Fig. 5.11 in absolute numbers) alone can not account for the peak at K7/M0. Fig. 5.10 also reveals that the fraction of fast rotators in the Hyades constantly grows towards cooler temperatures, rising clearly to over $50 \%$ for mid M-types, with detectable rotation in $100 \%$ of the latest type stars.

\subsubsection{Comparison to previous results}

One of the aims of this study was to establish a large catalog of rotation in the Hyades low-mass stars. To accomplish this, precedence for the new observations to the catalog were given to unexplored targets for which no data existed. However, a few of these new objects are covered in the literature with measured rotation rates, and we use them for the purpose of comparison with $v \sin i$ measured in this work (Fig. 5.12). In this figure, we plot $v \sin i$ values from previous works against those derived by our analysis. For most stars, there is good consistency within the uncertainties and detection limits. Note that in many cases, previous data has higher detection limits (6 to $10 \mathrm{~km} / \mathrm{s}$ ) than our new data, and that previous 


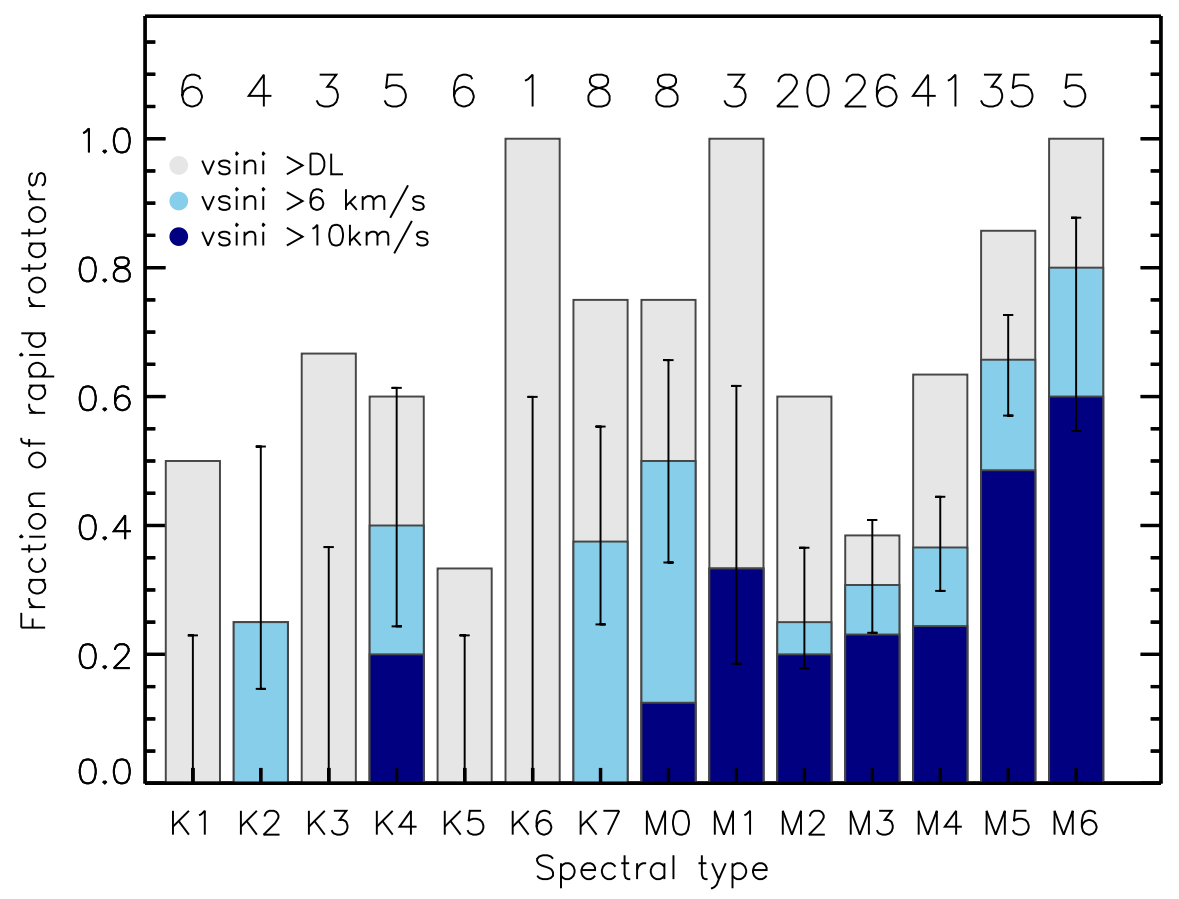

Figure 5.10.: Spectral type dependent ratios of rotating Hyades stars. Stars with rotational velocities $v \sin i$ above the detection limit $\left(3 \mathrm{~km} \mathrm{~s}^{-1}\right.$ in most cases) are plotted in the grey distribution. Moderate rotation $\left(v \sin i>6 \mathrm{~km} \mathrm{~s}^{-1}\right)$ is marked in light blue, rapid rotation $\left(v \sin i>10 \mathrm{~km} \mathrm{~s}^{-1}\right)$ in dark blue. Error bars refer to $1 \sigma$ uncertainties of the light blue distribution. Numbers on top of each bin give the total number of (rotating and non-rotating) stars per spectral type.

measurements rely on different methodologies. Table 5.4 lists those data. For the binaries in common (filled symbols in Fig. 5.12), there does not seem to be scatter much larger than for the other stars. Given that these binaries were observed at different epochs, one might expect to see a strong impact of different orbital phases on the resulting $v \sin i$, if binarity was a major agent of rotational broadening in these binaries. This is obviously not the case, and provides confidence that rotation rates can still be measured in binary stars. There may possibly be multiple stars in the sample for which, depending on orbital period and RV difference, rotational broadening will be deteriorated by the individual spectral line systems of the components (without being detected in the XCFs). However, such cases should remain statistical outliers and may be identified later by their unusually enlarged $v \sin i$ (see the discussion in Sec. 5.3.3).

There remain two objects in Fig. 5.12 with significant differences to previously reported rotation rates, that are not consistent within the uncertainties. One of them is $2 \mathrm{M} 04350255+0839304$, for which Stauffer et al. (1997a) reported a rotation rate more than twice of what our analysis suggests. At $v \sin i=25 \mathrm{~km} / \mathrm{s}$ we would easily identify the star as rotationally broadened in our XCFs. This is not the case. Instead, the XCFs are much narrower than what we see in faster rotating stars. No hints of a blended spectroscopic binary are detected that would argue for an additional broadening agent and lead to an overestimated rotation rate in Stauffer et al. (1997a). The second one is $2 \mathrm{M} 04322373+1745026$, for which we derive a $v \sin i 30 \%$ smaller than reported by Bleach et al. (2002b). We attribute this difference not to the binary 


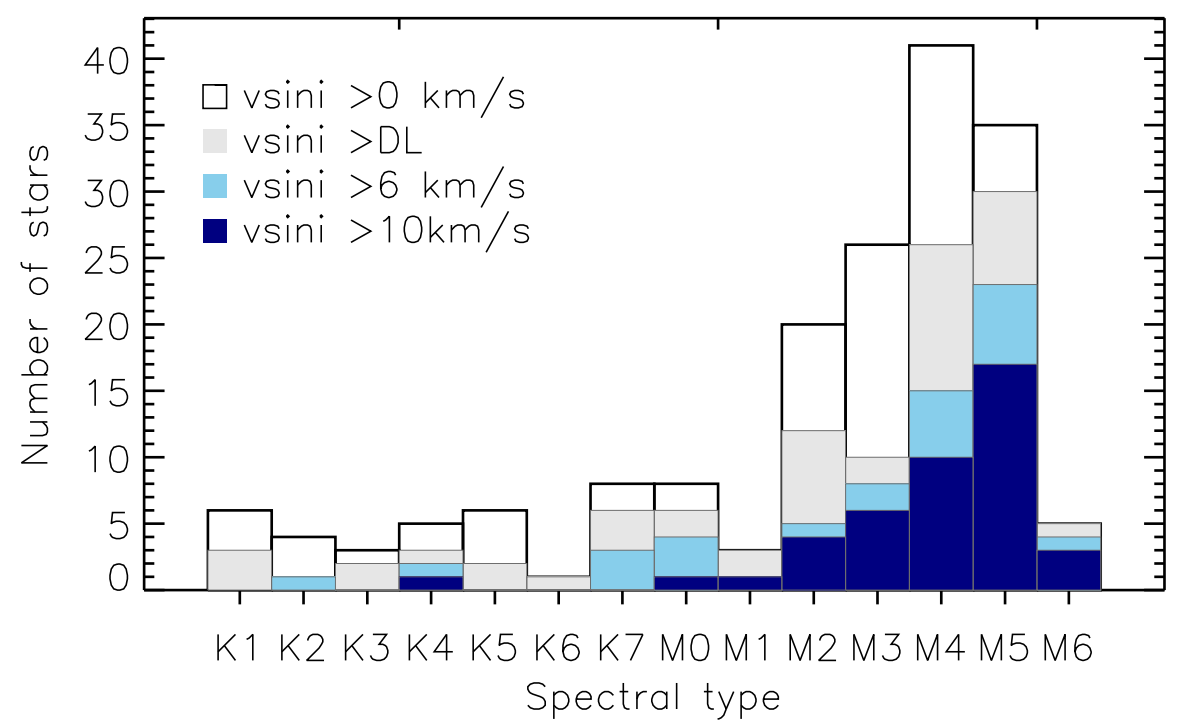

Figure 5.11.: Distribution of projected rotational velocities $v \sin i$ with spectral type. The open, outlined histogram comprises the entire set of rotating and non-rotating objects. Grey areas represent rotators with $v \sin i$ above the detection limit (DL) of the corresponding measurements (mostly, $\mathrm{DL}=3 \mathrm{~km} \mathrm{~s}^{-1}$ ). Rapid rotators with measured $v \sin i$ larger than $6 \mathrm{~km} \mathrm{~s}^{-1}$ and $10 \mathrm{~km} \mathrm{~s}^{-1}$ are indicated by light blue and dark blue histograms, respectively.

nature of the object, but to the very different method applied in Bleach et al. (2002b), and detailed in Sec. 5.1.

\subsubsection{Rotation-Activity relation}

Our catalog of Hyades $\mathrm{K}$ and $\mathrm{M}$ stars allows to probe stellar rotation and chromospheric and coronal activity from a large sample of common objects. This enables direct correlations between the observed consequences of the magnetic field generation and its most prominent driver. We show in Fig. 5.13 and 5.14 the correspondence of $v \sin i$ to $\mathrm{H} \alpha$ emission and Xray emission, respectively. As rotation is generally accepted to be a prerequisite of stellar magnetic fields (in a rotationally driven dynamo), and hence magnetic fields emanate in activity proxies, more rotation equates to stronger activity. The data clearly resembles this mechanism in both tracers, with the fastest objects being among the most active. Thus, the rotation-activity relation known from field stars (spanning the spectral type range F- down to $\mathrm{M}$ ) is still valid in the Hyades low-mass dwarfs, i.e. both in the slow/inactive K-dwarf and in the fast/active M-dwarf regime. There seems to be a limiting maximum of activity strength that these stars can produce, which is the same for all rotational velocities. This means that even among those stars, whose $v \sin i$ is too small to be detected, we find the same upper bounds of $\log \left(L_{\mathrm{H} \alpha} / L_{\mathrm{bol}}\right)_{\mathrm{sat}} \approx-3.5$ and $\log \left(L_{X} / L_{\mathrm{bol}}\right)_{\mathrm{sat}} \approx-2.5$ as for the most rapidly rotating stars in the sample. This saturation limit is seen in the same way than in old field stars (Schrijver \& Zwaan 2008; Pizzolato et al. 2003), albeit in the latter only at later spectral types. However, a few stars in the sample exhibit considerably stronger activity, argueably caused by powerful magnetic outbursts, and probably randomly caught during such events. One would expect that the most rapid individuals are, at the same time, also the most active 

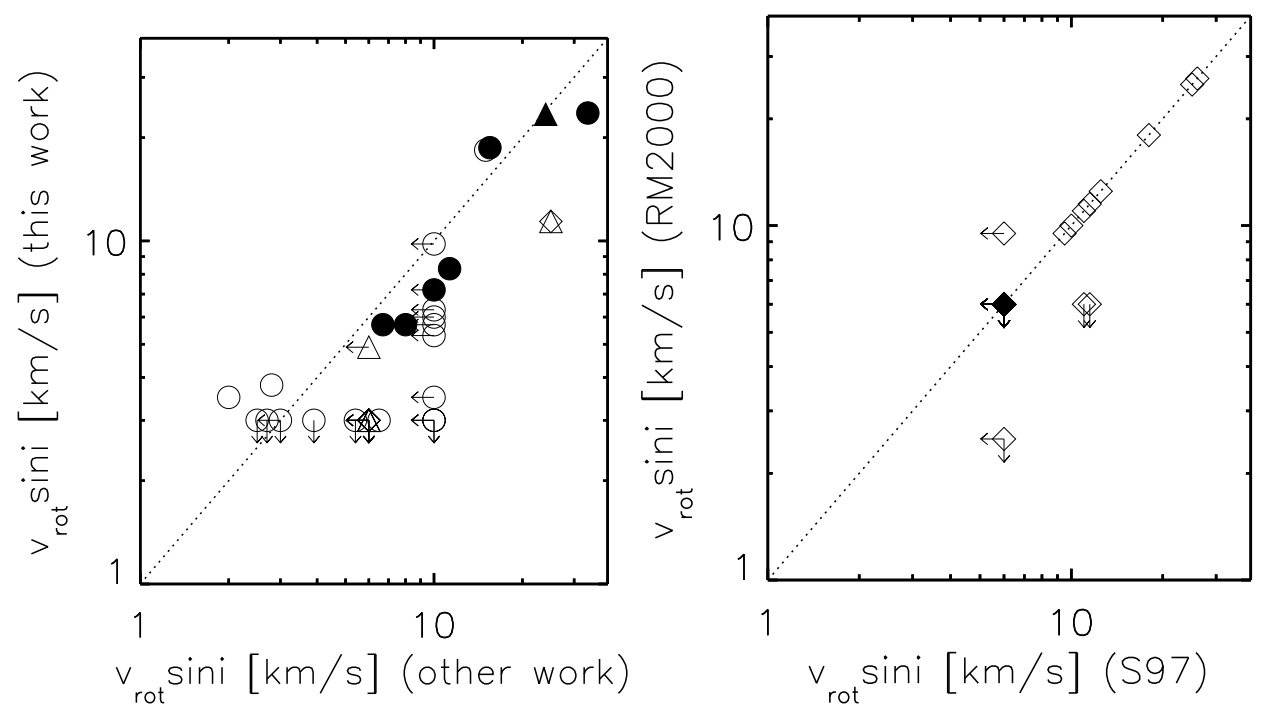

Figure 5.12.: Comparison of measured $v \sin i$ for objects in our sample with previously determined rotational velocities. Left: $v \sin i$ measured in this work versus values obtained by other studies. Stars included in S97 (Stauffer et al. 1997a) and RM2000 (Reid \& Mahoney 2000) are plotted as triangles and diamonds, respectively. Circles denote other works (for references, see Table 5.4). Filled symbols are confirmed binary systems. Right: comparison of $v \sin i$ for objects in common derived in S97 and RM2000. Note that stars on the identity line were only measured by S97, others were also remeasured by RM2000. See Fig. 5.8 for further explanations of plot symbols.

ones. While this is true for the general distribution in the sample, the three objects with $v \sin i>30 \mathrm{~km} / \mathrm{s}$ attract attention. Their $\mathrm{H} \alpha$ strength is slightly below $\log \left(L_{\mathrm{H} \alpha} / L_{\mathrm{bol}}\right)_{\mathrm{sat}}$. It must be noted though, that the corresponding coronal emission remains at very high levels.

There is a puzzling subset of targets in Fig. 5.13, however, that seems to contradict the rotation-activity connection. Eleven objects in the sample populate a range of rotational velocities $v \sin i \geq 6 \mathrm{~km} / \mathrm{s}$ (excluding upper limits), but surprisingly at very low or nondetected $\mathrm{H} \alpha$ emission strengths. One third of these objects are $\mathrm{K}$ stars, the others very early M-type. Even if we exclude the known binaries among them (33\%) for their potentially different evolution of rotation, the behaviour is still striking. The HAIRs are summarized in Table 5.3 with their properties. Interestingly, the same objects with detected rotation but with marginal or no $\mathrm{H} \alpha$ emission are not all inactive when we look at X-ray activity. There is only one star among the HAIRs, 2M 04181763+1500339, with a non-detection in $L_{X}$, the remaining showing $\log L_{X} / L_{\text {bol }}$ between -4.6 (rather inactive) and as high as -2.8 (close to $\log \left(L_{X} / L_{\text {bol }}\right)_{\text {sat }}$ ). Again, even if we exclude the binaries (which show $L_{X}$ above average among the HAIRs), strong X-ray emitters remain that are non-detections in $\mathrm{H} \alpha$. The strength of X-ray activity for the objects in question grows with decreasing effective temperature. One possible explanation is that all the HAIRs are hidden binaries which unidentifiably broadened the XCFs and so mimicked enhanced rotation. This would first require a small difference in $\mathrm{RV}$ and/or a much lower-mass companion, because otherwise the impact should be visible in the deformation of the XCFs (we have identified one object among the HAIRs, 2M 04412966+1313164, as a probable SB2 before; cf. Sec. 5.1). We estimate the 


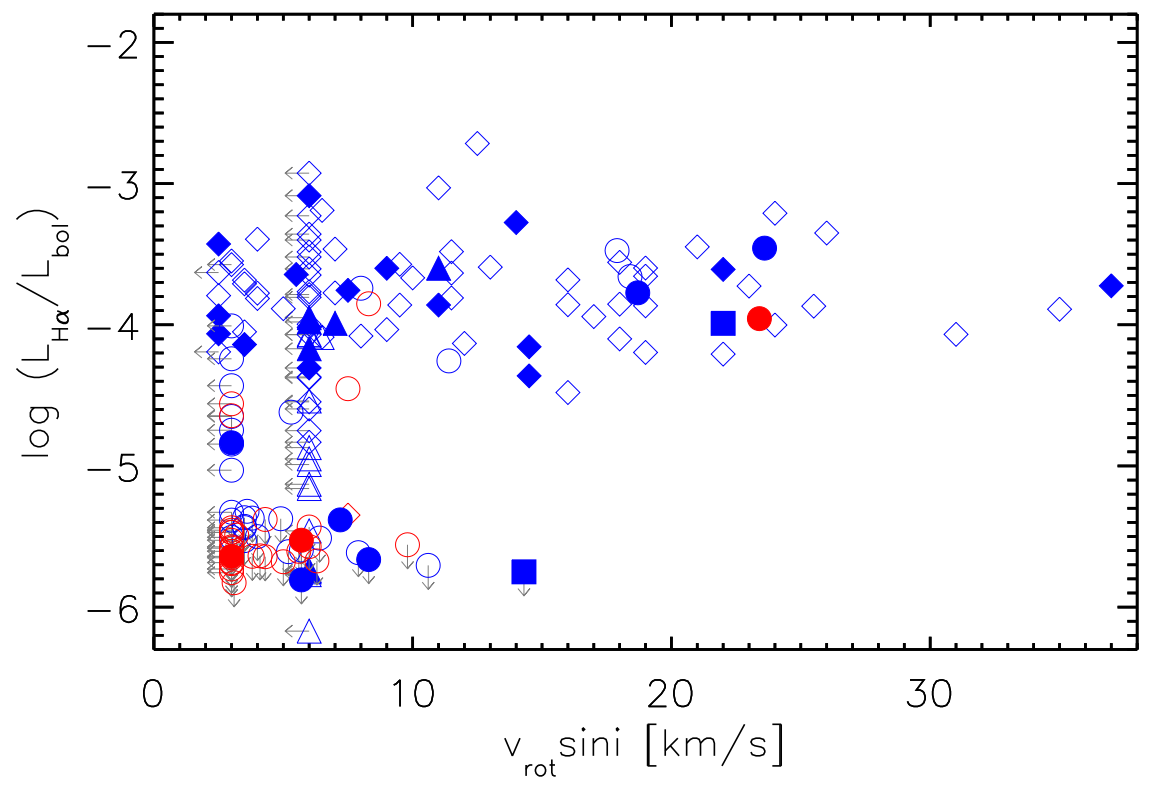

Figure 5.13.: Chromospheric activity versus rotational velocity $v_{r o t} \sin i$ for the Hyades $\mathrm{K}-$ $\mathrm{M}$ stars. Objects of spectral type $\mathrm{K}$ are denoted in red, M-type stars in blue. Upper (detection) limits are indicated by their respective arrows. For coding of symbols, refer to Fig. 5.3.

effect of such binary stars on the resulting $\mathrm{H} \alpha$ line profile, and find that for combinations of an earlier type star with $\mathrm{H} \alpha$ in absorption and a later type one with $\mathrm{H} \alpha$ in strong emission, together with a small enough $\Delta R V$, the line EW effectively vanishes or cancels out. We see indications of this mechanism in two of the binary HAIRs, namely 2M $04341113+1133285$ and 2M $04285080+1617204^{3}$, which may explain their positions in Fig. 5.13. Thus, one component in these objects should be active, but we are unable to disentangle them from the current epoch of data. The same may be true for 2M 04510241+1458167, the fastest presumably single HAIR, which also shows a high level of X-ray activity $\left(\log L_{X} / L_{\mathrm{bol}}=-3.5\right)$. Its $\mathrm{H} \alpha$ profile resembles both parts of an absorption and emission line, which leads us to the conclusion that this may indeed be a so far unnoticed binary star, with an unresolved but chromospherically active late-type companion. As a test, we try to reconstruct the $\mathrm{H} \alpha$ emission line of the active component in this case (assuming a superposed gaussian absorption profile). This yields a lower limit of $\log L_{\mathrm{H} \alpha} / L_{\mathrm{bol}} \gtrsim-4.8$ for $2 \mathrm{M} 04510241+1458167$ (as we do not know the bolometric luminosity of the active star, which should be lower), placing the object well towards the rotation-activity regime in Fig. 5.13, yet at considerably lower activity level than other stars with similar rotation ${ }^{4}$.

The remaining apparently single HAIRs do not bear indications of such hidden, active companions. Their $\mathrm{H} \alpha$ lines are moderate to strong absorption profiles, on display in Fig. 5.15. Any spectroscopic binary companion to these objects would have to show a significant flux contribution (ie. be within a few spectral types) in order to bias the measurement of $v \sin i$ (and not be at orbital phase close to zero), but this would mean a significant and measurable

\footnotetext{
${ }^{3}$ Another binary star, 2M 04480086+1703216, with $v \sin i=5.7 \pm 1.1$ not listed as a HAIR, reveals the same properties.

4 This estimate assumes that both components rotate with the same rotational velocity $v \sin i$, which is not necessarily the case.
} 


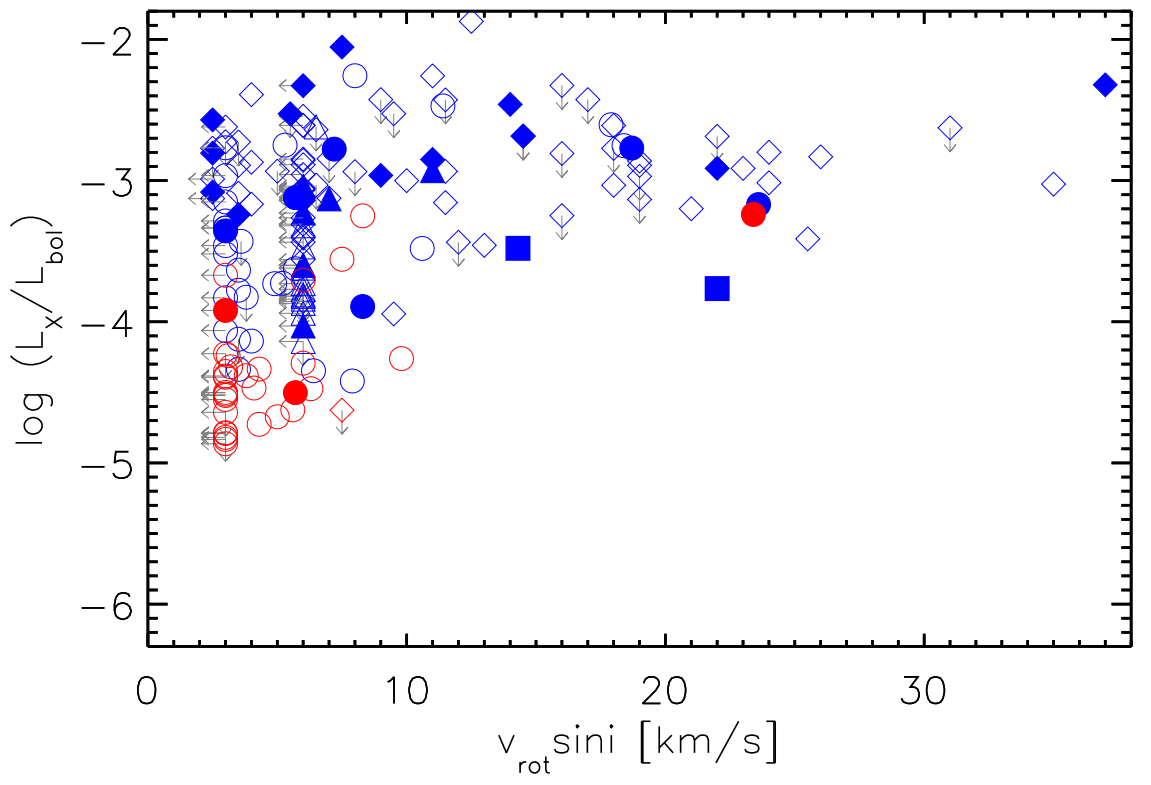

Figure 5.14.: Coronal activity versus rotational velocity $v_{\text {rot }} \sin i$ for the Hyades K-M stars. Colour coding and plot symbols as in Fig. 5.13. See Fig. 5.3 for further explanations of symbol shapes.

distortion of the resulting $\mathrm{H} \alpha$ profile (either emission or absorption). Or a companion would have an insignificant flux contribution, in which case $v \sin i$ would not be compromised and $\mathrm{H} \alpha$ might be disturbed (in case of emission) or not. Either scenario does not explain the observations. If we do not detect secondary $\mathrm{H} \alpha$ emission, then such a low-luminosity companion cannot be a contributor to the detected rotation either. This renders the binary hypothesis for the remaining HAIRs unlikely or irrelevant.

Furthermore, this would not explain the fact that the presumably single HAIRs do show Xray activity, but at levels around the lowest that we detect with $=-4.6 \leq \log L_{X} / L_{\text {bol }} \leq-4.3$ (there are three stars with higher values, $-3.7 \leq \log L_{X} / L_{\mathrm{bol}} \leq-3.6$, but they have lower $v \sin i \leq 6 \mathrm{~km} / \mathrm{s}$ ). This level is substantially below the average of confirmed binaries with detected rotation (around $\log L_{X} / L_{\mathrm{bol}}=-3.0$ ).

If the HAIRs do not form $\mathrm{H} \alpha$ emission lines in their chromospheres, these stars cannot have a high level of activity. With increasing activity level, $\mathrm{H} \alpha$ is known to increase in absorption before the line core fills in, and eventually turns into emission, while at the same time other activity tracers show an emission line from the beginning (Walkowicz \& Hawley 2009). We suspect that the HAIR stars might be in a phase where absorption is still present, but filling of the line with emission has not yet started (in terms of activity level). We therefore search these objects for emission in the Ca II H \& K lines. We find that all the stars show $\mathrm{H}$ and $\mathrm{K}$ with emission cores, while $\mathrm{H} \alpha$ is in absorption. Hence, indications are that the HAIR stars do still exhibit chromospheric activity, although another proxy for it, $\mathrm{H} \alpha$, does not show this. The level of activity in these stars is therefore comparably low, in concordance with a reduced level of coronal activity. In effect, the $\mathrm{H} \alpha$-inactive but rotating objects thus fall into two categories: the faster rotating among them $(v \sin i \leq 14 \mathrm{~km} / \mathrm{s})$ are likely binaries with at least one component showing activity, while the slowest detected rotators $(v \sin i \leq 8 \mathrm{~km} / \mathrm{s})$ without measurable $\mathrm{H} \alpha$ emission still show chromospheric activity as Calcium emission and coronal X-ray activity. In both categories, rotation is associated with signs of magnetic 
activity, so that the rotation-activity relation does not seem to be violated. Rotation thus remains a governing principle for magnetic activity throughout all spectral types $\mathrm{K}$ to $\mathrm{M}$, and moreover at younger ages. 

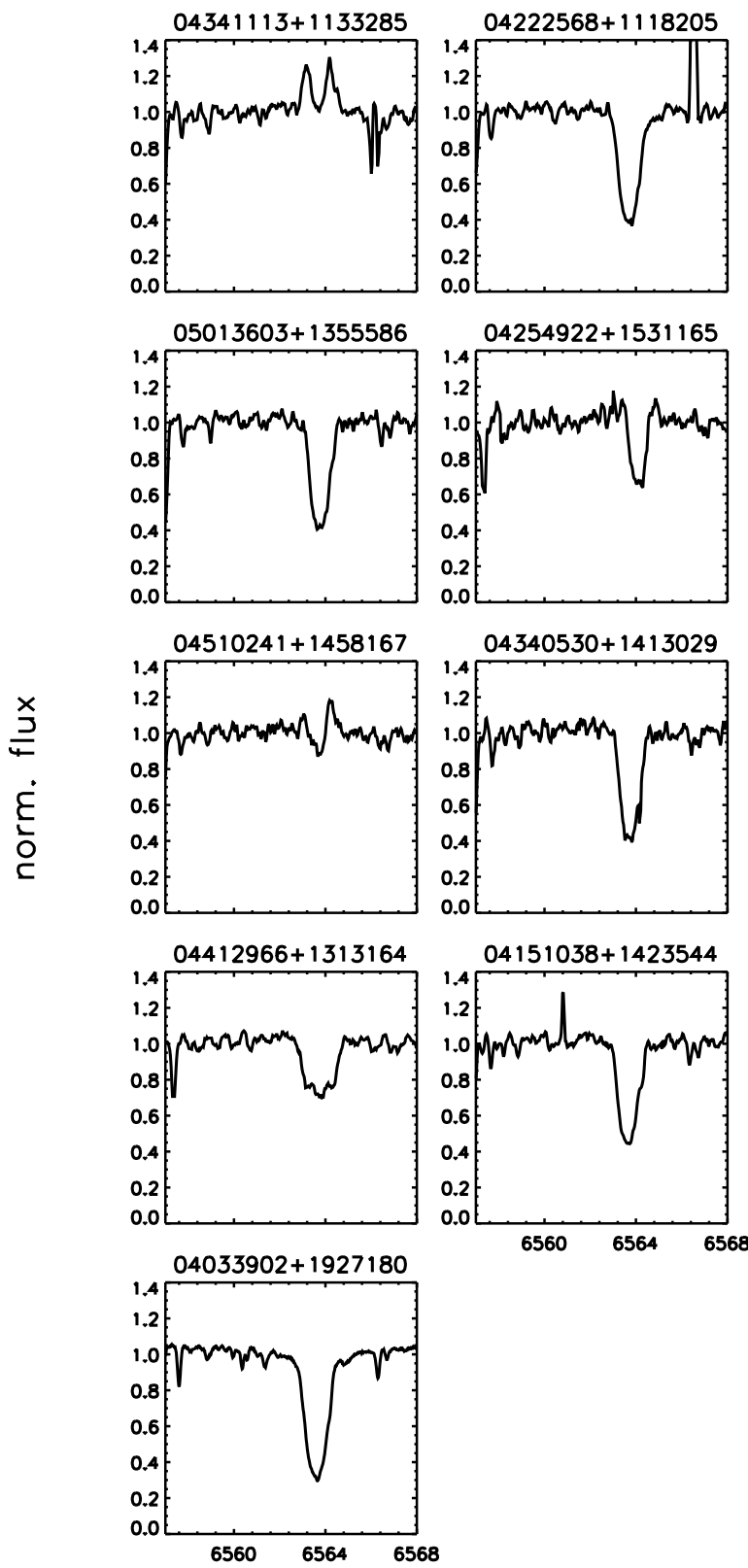

\section{Wavelength $[\mathrm{A}]$}

Figure 5.15.: $\mathrm{H} \alpha$ line profiles for nine of the eleven chromospherically inactive yet rotating stars from Table 5.3 (2M 04285080+1617204 and 2M 04181763+1500339 are not shown). 
Table 5.3.: Activity and rotation for $\mathrm{H} \alpha$ inactive yet rotating stars. For notes and references, please refer to Table A.1.

\begin{tabular}{|c|c|c|c|c|c|c|c|c|c|c|c|c|}
\hline $\begin{array}{l}\text { Object }^{a} \\
\text { 2MASS J }\end{array}$ & Other Name ${ }^{b}$ & $\begin{array}{l}T_{\text {eff }}{ }^{c} \\
{\left[{ }^{\circ} \mathrm{K}\right]}\end{array}$ & $\mathrm{SSpT}^{d}$ & & $\frac{\log L_{X}}{\log L_{\mathrm{bol}}}$ & & $\frac{\overline{\operatorname{og}} L_{H \alpha}}{\operatorname{og} L_{\text {bol }}}$ & $\begin{array}{r}v \sin i \\
{[\mathrm{~km}}\end{array}$ & $\begin{array}{l} \pm \sigma^{e} \\
\left.b^{-1}\right]\end{array}$ & SB? $f$ & $\operatorname{Ref}^{g}$ & Notes $^{h}$ \\
\hline $04341113+1133285$ & vB 294 & 3475 & M3 & & -2.78 & & -5.38 & 7.2 & \pm 1.2 & $\checkmark$ & $(9)$ & a) d) \\
\hline $04285080+1617204$ & vB 190 & 3690 & M1 & & -3.48 & $<$ & -5.75 & 14.3 & $\ldots$ & $\checkmark$ & (1) & a) d) \\
\hline $04222568+1118205$ & vB 259 & 3850 & M0 & & -3.89 & $<$ & -5.66 & 8.3 & \pm 1.1 & $\checkmark$ & (9) & a) d) \\
\hline $05013603+1355586$ & vB 348 & 3860 & M0 & & -4.42 & $<$ & -5.61 & 7.9 & \pm 1.4 & & (9) & a) d) \\
\hline $04254922+1531165$ & vA 366 & 3880 & M0 & & -4.35 & $<$ & -5.51 & 6.4 & \pm 1.1 & & (9) & c) f) \\
\hline $04510241+1458167$ & $\ldots$ & 3945 & M0 & & -3.48 & $<$ & -5.70 & 10.6 & \pm 1.4 & ? & (9) & c) e) \\
\hline $04340530+1413029$ & vA 731 & 4025 & K7 & & -3.71 & $<$ & -5.57 & 6.0 & \pm 0.8 & & (9) & a) d) \\
\hline $04412966+1313164$ & vB 316 & 4070 & K7 & & -4.26 & $<$ & -5.56 & 9.8 & \pm 1.5 & $?$ & (9) & c) d) \\
\hline $04151038+1423544$ & vA 72 & 4085 & K7 & & -4.29 & $<$ & -5.43 & 6.0 & \pm 1.0 & & (9) & a) d) \\
\hline $04033902+1927180$ & RHy 44 & 4625 & K4 & & -4.47 & $<$ & -5.67 & 6.3 & \pm 0.3 & & (9) & a) d) \\
\hline $04181763+1500339$ & RHy 200 & 4865 & $\mathrm{~K} 2$ & $<$ & -4.63 & & -5.35 & 7.5 & $\ldots$ & & (7) & c) h) \\
\hline
\end{tabular}


Table 5.4.: Comparison to other published rotational velocities $v \sin i$.

\begin{tabular}{|c|c|c|c|c|c|c|}
\hline \multirow[t]{2}{*}{ 2MASS J } & \multirow[t]{2}{*}{$\overline{\mathrm{SpT}}$} & \multicolumn{4}{|c|}{$v \sin i\left[\mathrm{~km} \mathrm{~s}^{-1}\right]$} & \multirow[b]{2}{*}{$\operatorname{Re}$} \\
\hline & & thi & work & & her & \\
\hline $04325009+1600210$ & K1 & & 5.7 & & 6.7 & $(2)$ \\
\hline $04181926+1605181$ & $\mathrm{~K} 2$ & $<$ & 2.5 & & 2.5 & (6) \\
\hline $04265434+1308175$ & $\mathrm{~K} 2$ & $<$ & 2.5 & & 5.4 & (6) \\
\hline $04395095+1243426$ & $\mathrm{~K} 2$ & $<$ & 2.5 & & 3.9 & (6) \\
\hline $04033902+1927180$ & $\mathrm{~K} 4$ & & 6.3 & $<$ & 10 & (3) \\
\hline $04322565+1306476$ & K4 & & 23.4 & & 24 & (4) \\
\hline $04235440+1403075$ & K4 & $<$ & 2.5 & $<$ & 3 & (6) \\
\hline $04070122+1520062$ & K5 & & 3.0 & & 6.5 & (6) \\
\hline $04333716+2109030$ & K5 & $<$ & 2.5 & & 2.7 & (6) \\
\hline $04334192+1900504$ & K6 & & 3.8 & & 2.8 & (6) \\
\hline $04412966+1313164$ & K7 & & 9.8 & $<$ & 10 & (3) \\
\hline $04063463+1332566$ & K7 & $<$ & 2.5 & $<$ & 6 & (4) \\
\hline $04340530+1413029$ & K7 & & 6.0 & $<$ & 10 & (3) \\
\hline $04161310+1853042$ & M0 & & 3.5 & & 2 & (6) \\
\hline $04222568+1118205$ & M0 & & 8.3 & & 11.3 & (6) \\
\hline $04244401+1046192$ & M0 & $<$ & 2.5 & & 6 & (4) \\
\hline $04285243+1558539$ & M0 & & 5.7 & $<$ & 10 & (3) \\
\hline $04480086+1703216$ & M2 & & 5.7 & & 8 & (1) \\
\hline $04291234+1516259$ & M2 & & 3.5 & $<$ & 10 & (3) \\
\hline $04060221+1815033$ & M2 & & 4.9 & $<$ & 6 & (4) \\
\hline $04360416+1853189$ & M2 & & 18.4 & & 15 & (3) \\
\hline $04350255+0839304$ & M3 & & 11.4 & & 25 & (4) \\
\hline $04341113+1133285$ & M3 & & 7.2 & $<$ & 10 & $(3)$ \\
\hline $04271663+1714305$ & M3 & $<$ & 2.5 & $<$ & 10 & (3) \\
\hline $04303385+1444532$ & M3 & & 3.0 & & 6 & (4) \\
\hline $04332699+1302438$ & M3 & & 3.0 & $<$ & 6 & (4) \\
\hline $04295572+1654506$ & M3 & & 2.8 & $<$ & 10 & (3) \\
\hline $04322373+1745026$ & M3 & & 23.6 & & 33.4 & $(5)$ \\
\hline $04251456+1858250$ & M4 & & 5.3 & $<$ & 10 & (3) \\
\hline $04290099+1840254$ & M4 & & 18.7 & & 15.5 & (3) \\
\hline
\end{tabular}

References. (1) Griffin et al. (1985); (2) Radick et al. (1987); (3) Stauffer et al. (1987); (4) Stauffer et al. (1997a); (5) Bleach et al. (2002b); (6) Mermilliod et al. (2009). 


\section{Discussion}

\section{Contents}

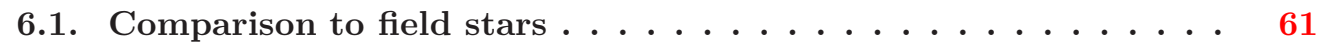

6.2. Comparison to rotation periods . . . . . . . . . . 64

6.3. Rotation and activity in binaries ............. 65

6.4. $\mathrm{H} \alpha$ inactive rotators . . . . . . . . . . . . 66

6.5. Is the rotation limit linked to the fully convective boundary? . . 67

\subsection{Comparison to field stars}

Our new data of the Hyades suggest that the fraction of fast rotators seen in the field is low in the mid-M range when compared to the younger Hyades stars. For the 625 Myr population, we find $30 \%$ of the M3 stars to be rapidly rotating, whereas this ratio is down to $15 \%$ for the field M3 stars. Fig. 6.1 illustrates our findings and compares the fractional rotation as seen in the field, and in our catalog. We plot as open squares data for the field stars adapted from Reiners et al. (2012, M0-M4) and Reiners \& Basri (2010, M7-M9), and include $1 \sigma$ statistical error bars drawn from the number of stars considered per spectral type bin. For the M5 and M6 bins, data is very limited (West \& Basri 2009b; Browning et al. 2010) and statistically not firm, so we do not plot these two bins (eg. in the sample by West \& Basri 2009b, there are only two stars of type M6, both fast rotators). It is important to realize that the field star distribution shows the ratio of stars rotating faster than $v \sin i>3 \mathrm{~km} / \mathrm{s}$, whereas this fixed threshold is not suitable for the Hyades. Other than in the field, a good fraction of Hyades in any spectral type bin, also among the "slow" early K-types, shows detectable rotation (ie. $v \sin i>3 \mathrm{~km} / \mathrm{s}$ in most cases; cf. Fig. 5.10), but clearly a change towards higher $v \sin i$ is observed for the later-type stars. An additional inconvenience is caused by the quickly dimishing stellar radius $R$ with respect to effective temperature among the M-dwarfs. $R$ decreases by a factor of 1.4 over the K-type range, but it does so by more than a factor of 5 across the M-types, hence the stellar surface velocities are vastly higher among the $\mathrm{M}$ stars and a direct comparison of $v \sin i$ across the $\mathrm{K}-\mathrm{M}$ range is difficult. Consequently, the matter of moment for the present discussion is the change from a slow level of rotation to an increased level of rapid rotation, with respect to the behaviour in field stars. To account for the radius effect, we derive rotation periods $P / \sin i=2 \pi R / v \sin i$ based on radii inferred from the BCAH models. These periods are still bound to the $\sin i$ ambiguity (a mean inclination of $i=90^{\circ}$ is assumed), so that $P$ is actually a lower limit. Rapid rotation in period space is considered for $P<3$ days, and the resulting distribution of rapid rotators is shown in Fig. 6.1 as starred symbols. Note that the distribution in case of the field stars in Fig. 6.1 does not significantly change when based on periods, as no K-types are covered.

It is striking that not only is the frequency of enhanced rotation lower in the field, but also we see a rise of rotation at earlier spectral types in the Hyades than in the field stars. No significant fraction of the old field stars (few Gyr) hotter than M3 are detected with rotation, 


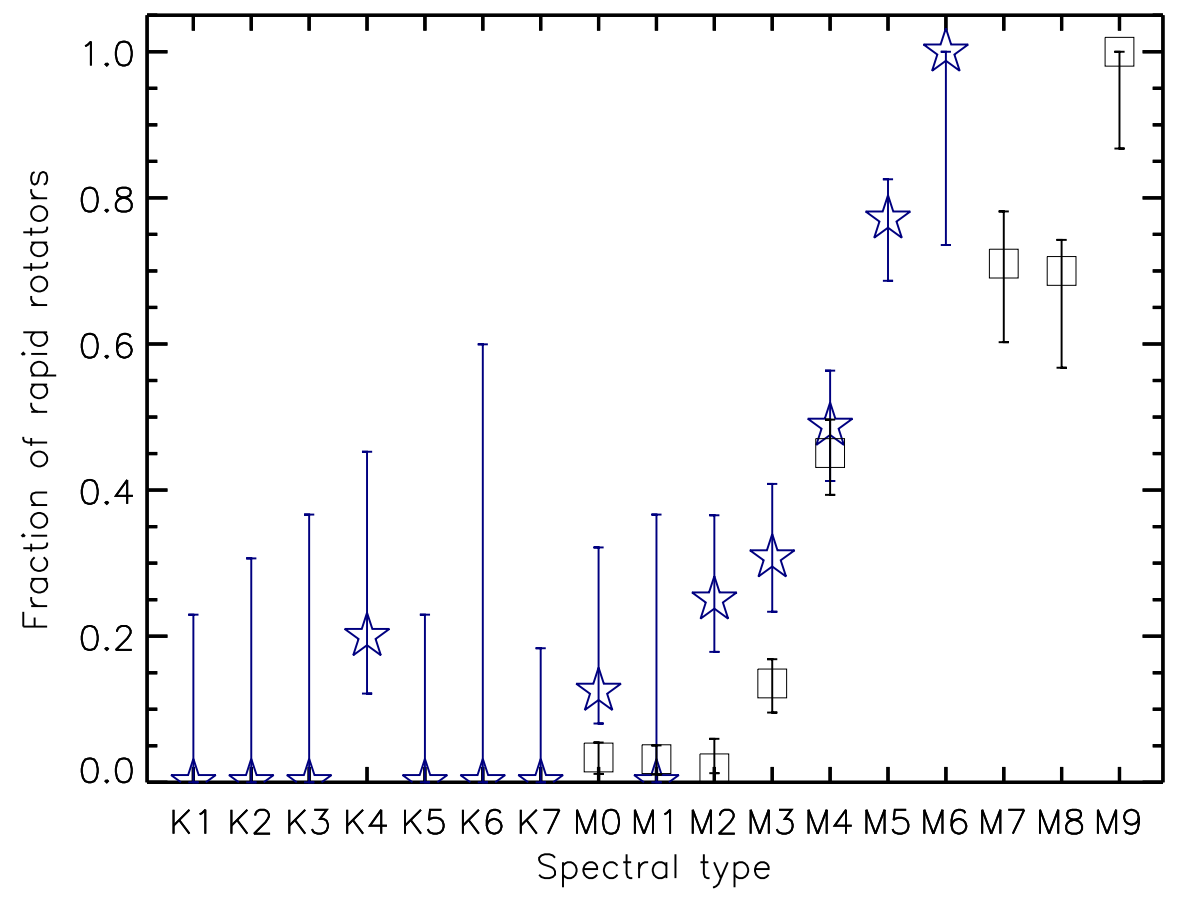

Figure 6.1.: Fraction of stars with detected rotation for the Hyades (this catalog, starred) and for field stars (squares). Data for the field stars adapted from Reiners et al. (2012, M0-M4) and Reiners \& Basri (2010, M7-M9). Error bars show statistical $1 \sigma$ uncertainties. For the Hyades, the fractions are expressed in rotational periods to eliminate the bias introduced by the stellar radius $R$, as $R$ quickly drops in this spectral type range.

whereas in the intermediate age Hyades (625 Myr) already more than $10 \%$ of the M0 stars are rapidly rotating, and $30 \%$ do so by M3. On the contrary, the field stars only begin to speed up notably at M3, and probably half of the field stars are rapid at M6, when already all of the cluster stars are observed rotating at high levels. Although no star in the M1 bin qualifies as rapid, we believe this is due to the low number of stars in that bin (three), and does not reflect a real paucity of increased rotation rate. An onset of rotation in the Hyades cluster thus starts as early-type as $\mathrm{K} 7-\mathrm{M} 0.12 \%$ of the M0 stars show fast rotation periods; or in terms of $v \sin i$, between $37 \%$ and $50 \%$ of M0 dwarfs rotate at a rate higher than $6 \mathrm{~km} / \mathrm{s}$ - this is not observed at higher temperatures,${ }^{1}$ - so the onset clearly occurs at higher massses than in the field.

At the same time, Fig. 6.2 makes clear, that when compared to the Gyr-aged field population, the $625 \mathrm{Myr}$ Hyades start to become also magnetically active at earlier spectral type. This activity dependence on stellar age has been first suspected by Stauffer et al. (1991) and further developed by Hawley \& Reid (1994) and Hawley et al. (1999), but the sampling remained sparse, and the locus of the $\mathrm{H} \alpha$-limit dilute. The new data in our catalog enables to refine this picture, providing further evidence for an $\mathrm{H} \alpha$-limit shifted to higher masses than in older stars (Fig. 6.2), and constraining its locus to spectral type K7/M0 (Fig. 5.6).

\footnotetext{
${ }^{1}$ Given the low number of stars in some K-type bins, particularly K6 and M1, the rotation onset in terms of spectral type has some uncertainty. However, rotation is clearly detected in stars typed M0 (see above). We also notice an increased $v \sin i$ (ie. below-average periods) in one K2 and one K4 star (cf. Fig. 5.10), though with low statistical significance (the rapid K4 rotator, vA 677, is discussed above, see Sec. 5.3).
} 


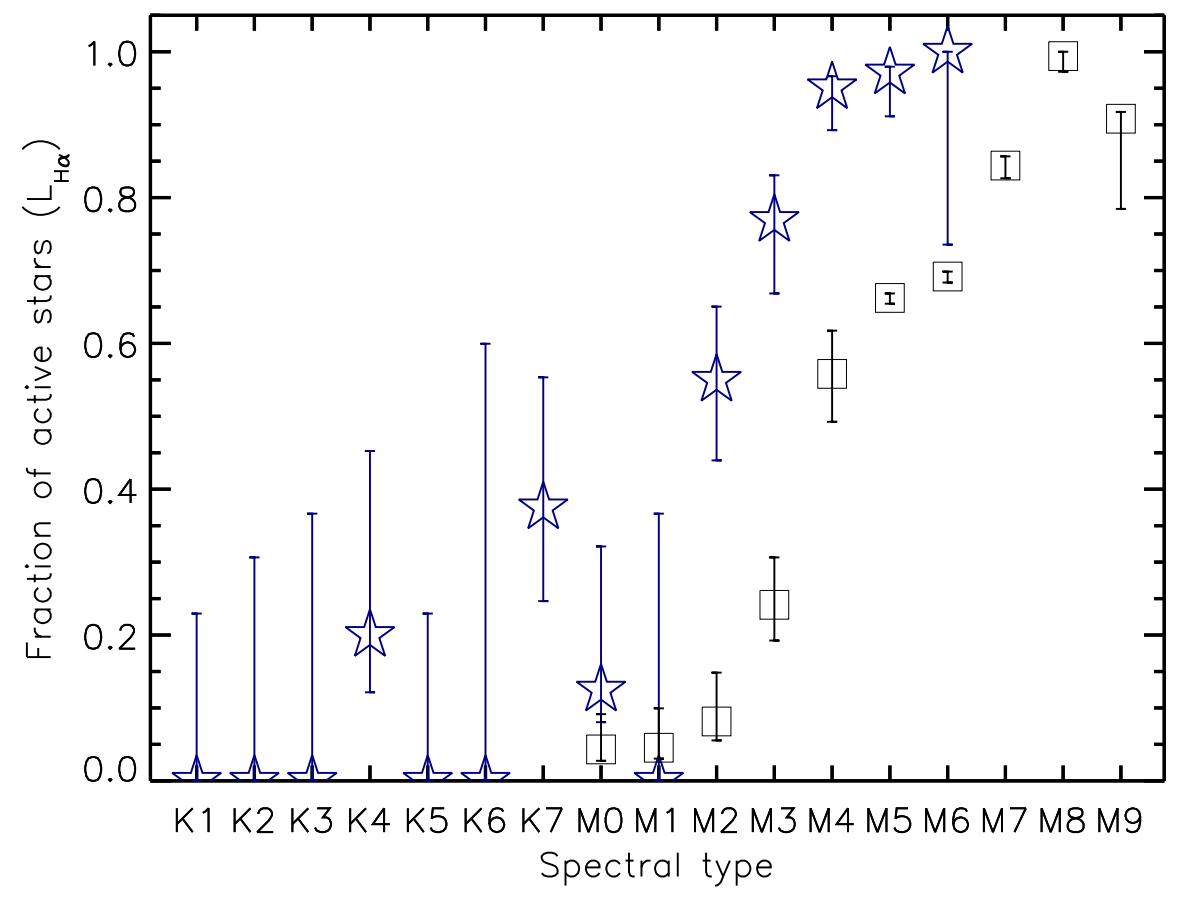

Figure 6.2.: Comparison of the fraction of stars with detected chromospheric activity in the Hyades and in field stars. Data from this catalog is shown as circles, field star data is taken from Reiners et al. (2012, M0 through M4) and West et al. (2008, M5 through M9), plotted as squares. Error bars show statistical $1 \sigma$ uncertainties.

The observed X-ray activity distribution (Fig. 5.7) shows virtually the same distribution, supporting the locus seen in $\mathrm{H} \alpha$.

Fig. 6.1 now provides a direct link to rotation, and supports the coexistence of an activitylimit and a rotation-limit, so that the locus of both prevalent activity and rapid rotation coincide. Thus, at the younger age of the Hyades, both rapid rotation and activity become predominant at higher masses than at older ages, and remain connected in the transition region. Hence, the rotation-activity correlation seems to be valid (in terms of mass) above, at, and below the $\mathrm{H} \alpha$-limit, though with different implications.

Our results in the Hyades cluster corroborate the idea that the highest mass at which $\mathrm{H} \alpha$ activity (or rapid rotation) is still predominant is a well defined function of stellar age. This is equivalent to a mass and age-dependent magnetic braking process, and the underlying braking efficiency function at a given age steepens at what is observed as the limiting $\mathrm{H} \alpha$ (or limiting rotation) mass. For other cluster ages, several determinations of the limiting $\mathrm{H} \alpha$ locus exist, eg. Hawley et al. (1999), and a few studies have reported indications of an onset also in rotation for younger clusters (eg. Stauffer et al. 1997b; Jackson \& Jeffries 2010b), or have noticed a possible drop-off in rotational periods (Lamm et al. 2005; Irwin et al. 2009; Delorme et al. 2011). The caveat at young ages of less than hundred Myr, however, is the high intrinsic spread of rotation rates. In these young clusters, the distribution of rotation has not yet converged onto an envelope of slow, effectively braked rotators. This is because the spin-up process emerging from the pre-MS contraction phase has not yet finished, leaving too little time for braking to take effect (even in the higher mass stars). Consequently, no massregime of converged, slow rotators (and thus inactive stars) yet exists, rendering a transition 
(activity-limit) difficult or impossible to discern.

The derived distribution of rotational velocities in the Hyades low-mass stars is controlled by considerable scatter in the levels of $v \sin i$. As the inclination angle $\sin i$ remains unknown, the projected rotation rates pose individual lower limits to the true velocities. To lift the degeneracy, photometric periods are needed (see below) - but mostly not available for lowmass Hyades. However, there is no reason to assume that the inclination angle is not randomly oriented in a cluster like the Hyades (Jackson \& Jeffries 2010a), so that a systematic bias resulting from restrictions on $\sin i$ is not expected. Thus, individual stars may be off from their true value of rotational velocity, but this is not the case for the distribution of $v$ over the entire sample with random inclinations (cf. estimates in Reiners et al. 2012). The scatter in observed rotation rates should therefore map the real distribution, so that a range of volocities exists especially among the M-dwarfs, where most stars are seen rapid (Fig. 5.12). In turn, it also means that the majority of stars in which no or little rotation is observed, still rotate slightly above the detection limit (cf. light grey distribution in Fig. 5.12). This basal level of rotation in the Hyades K-dwarfs is consistent with an age-effect: Magnetic braking over the age of the cluster has not yet brought these stars to the same slow level of rotation that is observed in old field K-dwarfs. The spin-down time span for this spectral type thus must be of order, but slightly longer than the $625 \mathrm{Myr}$ of the Hyades cluster.

\subsection{Comparison to rotation periods}

We compare the available rotational periods for the Hyades with our own measurements, derived as described above, in Fig. 6.3. Again, we do not assume a mean inclination angle, so our periods are still prone to $\sin i$ and thus are technically lower limits. Photometric periods are compiled from Radick et al. (1987), Prosser et al. (1995), and Delorme et al. (2011). We also include in Fig. 6.3 period measurements for the Praesepe open cluster, which is nearly coeval $(\approx 600 \mathrm{Myr}$; Irwin et al. 2009) to the Hyades and thus should show a distribution of rotation with mass very akin to that of the slightly older Hyades. Rotation periods for Praesepe are taken from Scholz \& Eislöffel (2007), Scholz et al. (2011), and Delorme et al. (2011). $T_{\text {eff }}$ is based on $(B-V)$ photometry supplied by Radick et al. (1987) and Prosser et al. (1995), which is transformed analogous to Sec. 4.1 (these two papers provide only very few M-stars). In the case of Praesepe, Scholz \& Eislöffel (2007) and Scholz et al. (2011) have determined masses (almost all of them M-dwarfs) from absolute $I_{C}$ and $J$ photometry given a cluster distance of $170 \mathrm{pc}$, and applying the $\mathrm{BCAH}$ mass-magnitude relations (which also yields $T_{\text {eff }}$ from the models). For the Delorme et al. (2011) data (both Hyades and Praesepe), we chose not to use their masses derived from $V-K$ photometry, but to go for absolute $M_{K}$ magnitudes $^{2}$ and utilize the BCAH $T_{\text {eff }}-M_{K}$ relationships (see Sec. 4.1, and the discussion in Reiners \& Mohanty 2012, about discrepancies in mass resulting from $V-K$ as provided by Delorme et al).

Fig. 6.3 shows good agreement between the photometric rotation periods and those derived from our $v \sin i$ measurements. First, both distributions nicely resemble the envelope of slow rotation, with a mean period of $\approx 10$ days for stars of type mid-K. The long period envelope seems to be slightly inclined with respect towards the higher mass end, reflecting the fact that at 600-650 Myr, higher mass solar-like stars have not yet slowed down to the level of their lower mass (still solar-like) counterparts. Note that our $v \sin i$ data cannot reproduce the "hump"

\footnotetext{
${ }^{2}$ We assume a cluster distance of 45 pc (Hyades) and 170 pc (Praesepe), as only few Delorme et al. (2011) objects have parallaxes.
} 
of slow rotators $(P>10 \mathrm{~d})$ for M0-M3 stars. This is because these slow periods correspond to $v \sin i<3 \mathrm{~km} / \mathrm{s}$ and are beyond the detection limit of our survey (indicated by a dashed line in Fig. 6.3). There seems to be a sharp drop among the longest periods in the M0-M3 range, so that the envelope of slow rotators falls to $\approx 6 \mathrm{~km} \mathrm{~s}^{-1}$ at $T_{\text {eff }}=3500 \mathrm{~K}$. This is not an observational bias, but can be explained by core-envelope decoupling (eg. Denissenkov et al. 2010). Invoking this mechanism, the total stellar spin-down time is thought to lengthen as only the convective shell is braked efficiently by angular momentum loss, while the radiative core maintains its spin for longer times, i.e. is decoupled. The "hump" thus resembles the slow surface rotation of the outer convection layer, while the data is not sensitive to the core rotation. Core-envelope decoupling cannot work for fully convective stars, which defines the upper edge of slow periods at spectral type M3.

Likewise dictated by the (solar-type) rotation-age relation, the slowest rotators at $600-$ $650 \mathrm{Myr}$ at the highest $T_{\text {eff }}$ considered here $(P \approx 10 \mathrm{~d}$ at $5200 \mathrm{~K})$ are still spinning considerably faster than Gyr aged counterparts in the field and the present day sun. The seven largest periods that we observe in our catalog of $v \sin i$ (corresponding to $12<P / \sin i<14$ days) seem to be slightly longer than what is suggested by the literature rotation periods $(P \approx$ 10 days). As we assumed $i=90^{\circ}$, their true periods may be lower given the unknown $\sin i$. Additionally, considering the error margins of these data points in $v \sin i$ space, the resulting (projected) periods are still consistent with the slow envelope laid out by the photometric periods at these masses.

Second, the lower bound in periods given by the rapid rotators also matches well across the range of spectral types. Note that the data from Delorme et al. (2011) is limited to $1.1<P<$ 20 days, but only covers spectral types in Fig. 6.3 for which periods are expected inside this range. ${ }^{3}$ So, the ranges of rotation rates present in Fig. 6.3 in each spectral type, observed in $P$ (from photometry) and in $P / \sin i$ (from $v \sin i$ ) are very similar (except, naturally, for the "hump" of long periods as mentioned above, which cannot be resolved in $v \sin i$ ). This means that the assumption of randomly orientated inclinations $i$ is consistent in our sample, and that statistically $i$ does not introduce systematic effects. The scatter observed in projected rotational velocities is thus real, meaning it reflects the true range of periods, and is not caused by a scatter in inclinations.

\subsection{Rotation and activity in binaries}

In the present study, we have identified 44 binaries and binary candidates among the stars in our catalog. Their behaviour in rotation and activity follows the general trends seen in the other (single) objects. Multiple systems lie within the scatter of activity levels in Xray and $\mathrm{H} \alpha$, and no obvious trend towards different rotation rates than the single stars is observed (individual exceptions are discussed in Sec. 5.3). Given that the majority of these binaries are identified as SB2s or from direct imaging, it is evident that their detection is biased towards massive short-period companions and wide systems with long orbital periods, respectively. Although our binary fraction is likely not complete, we see that companions in wide orbits likely behave as do independent single stars. In contrast, we have identified pairs of binary stars with measured close separation and short orbital periods, and these objects

\footnotetext{
${ }^{3}$ There may possibly be a few individual rapid rotators missed by the limited range in $P$ in the Delorme et al. (2011) data among the K-dwarfs, and likewise around the 20 days end at lower masses, but these missing points would not change the distribution of periods. Data from these authors does not extend beyond masses/spectral types where $P$ slows down to below 1.1 days, but this regime is well sampled by the Praesepe data from other sources (Scholz \& Eislöffel 2007; Scholz et al. 2011).
} 


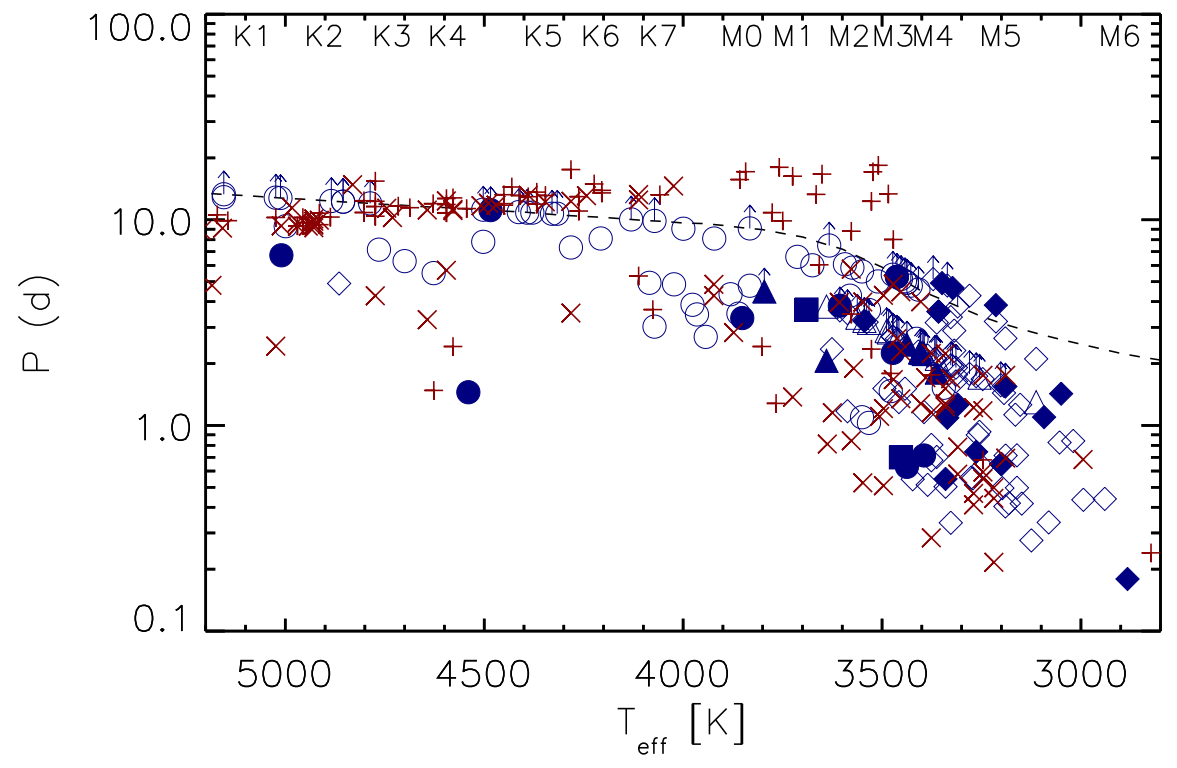

Figure 6.3.: Comparison of rotational periods derived in this work with photometric periods from the literature for the nearly coeval Hyades and Praesepe clusters. Pluses (Hyades) and crosses (Praesepe) denote data from Radick et al. (1987); Prosser et al. (1995); Delorme et al. (2011) and Scholz \& Eislöffel (2007); Scholz et al. (2011); Delorme et al. (2011), respectively. Other symbols mark Hyades data from this catalog as in previous figures, with single stars as open, and binaries as filled symbols. The dashed line indicates our corresponding $v \sin i$ detection limit (and hence an upper limit) of $v \sin i=3 \mathrm{~km} \mathrm{~s}^{-1}$.

stand out in both activity indicators, and also show enhanced rotation rates where their single counterparts do not. Recently, Rebassa-Mansergas et al. (2012) have demonstrated for white dwarf binaries with low-mass companions, that enhanced rotation and activity can be expected from such close systems independently of spectral type. That is, companions of any spectral type in these close systems are prone to much higher rotation rates, and to increased activity, than single stars of the same spectral type. The reason for this mechanism is likely tidal locking. On the other hand, companions in wide systems act like individually evolved stars. Our analysis adds evidence for this scheme for at least two binaries (only one of them a PCEB, comprising a WD), namely 2M 04322565+1306476 and 2M 04322373+1745026, with orbital periods below two days. We argue that these systems are tidally locked, explaining the increased rotation rates for the given spectral types. Their enhanced activity further supports the universal concept of rotationally driven activity, so that increased rotation always gives rise to enhanced magnetic activity.

\section{4. $\mathrm{H} \alpha$ inactive rotators}

In our catalog of low-mass Hyades, we detect a number of objects with rotation rates $v \sin i \geq$ $6 \mathrm{~km} / \mathrm{s}$ that do not show signs of activity in $\mathrm{H} \alpha$. These HAIRs are observed with $\mathrm{H} \alpha$ lines in absorption, in contrast to the expectation that significantly rotating stars are also active at the same time (which we find for the other sample stars). While a few of these objects can be explained by binary stars in which one component outshines the other, rendering the true 
$\mathrm{H} \alpha$ emission flux hard to detect, this cannot be the case for all. These HAIR stars appear as inactive or moderately active in X-ray activity, and at the same time show chromospheric activity in the form of Calcium $\mathrm{H}$ and $\mathrm{K}$ emission cores. It is intriguing that this behaviour, in a few stars, extends over temperatures that correspond to the entire range of spectral class $\mathrm{K}$, and into the M-type regime. In turn, this means that $\mathrm{H} \alpha$ emission alone might not be a universal indicator of magnetic activity over this entire spectral type range. If $\mathrm{H} \alpha$ absorption can still be visible in the earliest active M-dwarfs, the filling-in of the $\mathrm{H} \alpha$ line (Cram \& Mullan 1979) must be very subtle with increasing activity strength until at some point it noticably turns into emission.

We conclude that a direct correspondence of $\mathrm{H} \alpha$ emission and eg. Ca II $\mathrm{H} \& \mathrm{~K}$ emission as seen in more active stars (Walkowicz \& Hawley 2009) is weak and not necessarily unique in the temperature range discussed here. In other words, several activity diagnostics respond with quite different sensitivities to low to mild levels of magnetic activity, and the absence of a particular emission feature does not necessarily exclude the presence of magnetic activity. As a consequence, magnetic activity of rather weakly $\mathrm{H} \alpha$ active stars of spectral type $\mathrm{K}-\mathrm{M}$ might be underestimated for objects with $\mathrm{H} \alpha$ in absorption. This is not a concern for the present study, but may help to understand the scatter in the coronal-chromospheric activity relation (Fig. 5.5) towards the lowest values of $\log L_{\mathrm{H} \alpha} / L_{\mathrm{bol}}$.

\subsection{Is the rotation limit linked to the fully convective boundary?}

One of the goals of the present work was to test whether the transition from slow to rapid rotation (with respect to effective temperature/mass) in the Hyades is tied to the boundary to full convection. As in field stars the rotation transition, as pointed out in Fig. 6.1, coincides with the convection boundary at spectral type M3 $\left(0.3 M_{\odot}\right)$, it has been suggestive to identify the latter as the cause of the former. As we have shown, the distribution of rotation in the Hyades low-mass stars is clearly distinct from that of the field star population. From Fig. 6.1 it is evident that the transition from the plateau of slow rotation rates to the domain of rapid rotators is clearly ocurring at ealier spectral types (beginning at around M0, completed by M6) at 625 Myr than at several Gyr (starting at around M3, probably complete by M9). At the same time, all stars with masses $1-0.1 M_{\odot}$ have converged onto the MS by $\approx 600 \mathrm{Myr}$. Consequently, they have all stopped spinning up during their pre-MS phase, and are only facing brake-down by angular momentum loss as time evolves. More importantly, the stellar mass at which a radiative core has already formed is not a function of time anymore (highermass stars form radiative cores earlier, and have completed doing so by around $50 \mathrm{Myr}$ to $130 \mathrm{Myr}$ for $1-0.6 M_{\odot}$ and $\gtrsim 0.35 M_{\odot}$, respectively). Stars with $\lesssim 0.35 M_{\odot}$ never form radiative cores and thus are always fully convective. Hence, the fully convective boundary in the 625 Myr Hyades stars is fixed to the same mass as in older field stars.

Effectively, our results thus show that the rotational transition does not coincide with the fully convective boundary, in contrast to older stars. This adds further support that a changing magnetic dynamo across the convective boundary does not play a governing role in the evolution of rotation, and for the observed break in rotation. Our results ultimately encourage models of angular momentum evolution that can explain the observed rotation rates for all masses without the need to invoke changes in the magnetic field structure, resulting from different dynamo mechanisms (eg. Reiners \& Mohanty 2012). The processes of magnetic field production thus do not seem to shape the lengthening of spin-down times in M-dwarfs. 



\section{Summary}

In this part of the thesis, we present the to date largest comprehensive catalog of rotation and activity in the low-mass Hyades, derived from new observations and from the literature. The catalog comprises simultanous measurements of projected rotational velocities and chromospheric activity, combined with coronal X-ray activity data, for all stars. The large sample of $171 \mathrm{~K}$ and $\mathrm{M}$ stars covers spectral types on both sides of the transition region between partial and full convection. We identified 33 binaries throughout the sample where confirmations were available, and additionally discovered five new spectroscopic binaries among the new high-resolution observations.

Our investigation of rotation and activity in partially convective and fully convective Hyades low-mass stars shows, that both activity and rapid rotation become prevalent in early M-type stars, while earlier K-type stars are inactive and slow rotators. This is a situation very similar to what is observed in older field stars, however, in contrast to field stars the younger Hyades show a different transition between these two regimes. At ages of several Gyr, the transition from the non-rotation to the rapid rotation dominated regime is found around spectral class M3. At ages of $625 \mathrm{Myr}$, our analysis shows for the Hyades that this transition occurs at earlier spectral type, ie. around K7/M0.

We find that the behaviour in terms of magnetic activity follows the transition in rotation. This changeover from inactivity to activity predominance (termed " $\mathrm{H} \alpha$ limit") occurs at the same spectral type around K7/M0 as found for rotation, thus the behaviour of rotation and activity with respect to spectral type (or mass) is very similar. We conclude from these findings that rotation and activity are tightly linked, hence the rotation-activity relation remains valid a) at the age of the Hyades, and b) both beyond and below (in terms of mass) the transition to prevalent activity/rotation. While earlier-type stars until K7/M0 have already slowed down rotation considerably during their lifetime, presumably due to magnetic braking, i.e. angular momentum loss via stellar winds, we find evidence that these stars in our sample are on average still spinning at higher rates than corresponding field stars. Angular momentum loss in these cluster stars has operated to a lesser extent compared to field stars of the same spectral types, which exhibit slower rotation periods. Spin-down timescales for stars of spectral class $\mathrm{K}$ must thus exceed the $625 \mathrm{Myr}$ age.

In the later-type Hyades $(\approx \mathrm{M} 0$ down to spectral type $\mathrm{M} 6)$ both rotation and activity remain at high rates for much longer times. In these types, magnetic braking efficiency decreases rather quickly with decreasing mass, leaving stars at these masses at increasingly higher average rotation rates than their earlier-type counterparts (ie. earlier than M0 in the Hyades, earlier than M3 in the field). The difference between our sample and the field then arises from the duration of the angular momentum loss. Since the agents of both angular momentum spin-up and magnetic braking efficiency are thought to be mass-dependent, the shape of the rotation-mass relation (more precisely, the frequency of rapid rotation as a function of mass or temperature; Fig. 6.1), is not simply shifted to lower values (longer braking duration) when comparing $625 \mathrm{Myr}$ with Gyr field stars, but also smeared out to earlier spectral type (mass-dependent braking).

An important result is that the locus of the rotation limit, ie. the transition from slow 
rotation to rapid rotation rates, does not seem to coincide with the fully convective boundary at spectral type $\mathrm{M} 3\left(\approx 0.3 M_{\odot}\right)$. This finding is consistent with the idea that a changing magnetic dynamo mechanism at the transition to full convection is not a major ingredient to explain the rotational evolution of low-mass stars and the observed break in rotation. Our results support the view that a change in the way magnetic fields are generated, and the occurence of rapid rotation in low-mass stars are processes that are not tightly linked.

Our results also put constraints on the quest for extrasolar planets in open clusters such as the Hyades. Planet searches in clusters have so far gained little success, with only few findings compared to the vast number of planets detected around nearby (field) stars. Effort is being made especially on low-mass planets around M-stars, as their detection is favourable around smaller and lower-mass stars. It is crucial to know the line broadening by rotation and the activity and its variability of the target stars, because these agents dramatically limit the planet detection sensitivity. Our catalog thus can serve as a valuable input in shaping the target selection for planet search programmes. 
Appendices 


\section{A. Results on rotation and activity in the Hyades}


Table A.1.: Activity and rotation results for the Hyades combined catalog.

\begin{tabular}{|c|c|c|c|c|c|c|c|c|c|c|c|c|c|}
\hline $\begin{array}{l}\text { Object }^{a} \\
\text { 2MASS J }\end{array}$ & Other Name ${ }^{b}$ & $\begin{array}{l}T_{\mathrm{eff}^{c}} \\
{\left[{ }^{\circ} \mathrm{K}\right]}\end{array}$ & $\mathrm{SpT}^{d}$ & & $\frac{\log L_{X}}{\operatorname{og} L_{\text {bol }}}$ & & $\frac{L_{H \alpha}}{L_{\mathrm{bol}}}$ & & $\begin{array}{l}v \sin i= \\
{[\mathrm{km} \mathrm{s}}\end{array}$ & & $\mathrm{SB}^{f}$ & $\operatorname{Ref}^{g}$ & Notes $^{h}$ \\
\hline $03000280+0744590$ & $\mathrm{BD}+07459$ & 5155 & K1 & & -4.24 & $<$ & -5.83 & & 3.1 & \pm 0.9 & & $(9)$ & c) e) \\
\hline $04092011+0807436$ & HG 7-113 & 5155 & K1 & & -4.79 & $<$ & -5.46 & $<$ & 3.0 & \pm 1.0 & & (9) & a) d) \\
\hline $03481186+0708464$ & LP 533-57 & 5025 & K1 & & -4.86 & $<$ & -5.53 & $<$ & 3.0 & \pm 0.4 & & (9) & a) d) \\
\hline $04325009+1600210$ & vB 91; vA 684 & 5010 & K1 & & -4.50 & $<$ & -5.53 & & 5.7 & \pm 0.9 & $\checkmark$ & (9) & a) d) \\
\hline $05004888+0443591$ & $\mathrm{BD}+04810$ & 5010 & K1 & $<$ & -4.64 & $<$ & -5.65 & $<$ & 3.0 & \pm 0.1 & & (9) & a) h) \\
\hline $04345007+2023404$ & LP 415-1399 & 5000 & K1 & & -4.47 & $<$ & -5.64 & & 4.1 & \pm 1.1 & & (9) & a) d) \\
\hline $04181926+1605181$ & vB 25 & 4885 & $\mathrm{~K} 2$ & & -4.55 & $<$ & -5.44 & $<$ & 3.0 & \pm 0.3 & & (9) & a) d) \\
\hline $04181763+1500339$ & RHy 200 & 4865 & $\mathrm{~K} 2$ & $<$ & -4.63 & & -5.35 & & 7.5 & $\ldots$ & & (7) & c) h) \\
\hline $04395095+1243426$ & vB 311 & 4855 & $\mathrm{~K} 2$ & & -4.52 & $<$ & -5.60 & $<$ & 3.0 & \pm 0.2 & & (9) & a) d) \\
\hline $04265434+1308175$ & LP 415-75 & 4855 & $\mathrm{~K} 2$ & & -4.79 & $<$ & -5.57 & $<$ & 3.0 & \pm 0.2 & & (9) & a) d) \\
\hline $04082667+1211304$ & vB 233 & 4785 & K3 & & -4.83 & $<$ & -5.47 & $<$ & 3.0 & \pm 0.8 & & (9) & a) d) \\
\hline $04275674+1903390$ & HG 7-227 & 4765 & K3 & & -4.67 & $<$ & -5.68 & & 5.0 & \pm 0.9 & & (9) & a) d) \\
\hline $04500069+1624436$ & Pels 96 & 4700 & K3 & & -4.62 & $<$ & -5.60 & & 5.6 & \pm 1.1 & & (9) & a) d) \\
\hline $04033902+1927180$ & RHy 44 & 4625 & K4 & & -4.47 & $<$ & -5.67 & & 6.3 & \pm 0.3 & & (9) & a) d) \\
\hline $04322565+1306476$ & vB 288; vA 677 & 4540 & K4 & & -3.24 & & -3.96 & & 23.4 & \pm 1.2 & $\checkmark$ & (9) & a) d) \\
\hline $04235440+1403075$ & vB 262; vA 294 & 4500 & K4 & & -4.38 & $<$ & -4.64 & $<$ & 3.0 & \pm 1.4 & & (9) & a) d) \\
\hline $04473532+1453209$ & vB 332 & 4500 & K4 & & -4.73 & $<$ & -5.64 & & 4.3 & \pm 0.5 & & (9) & a) d) \\
\hline $04145191+1303178$ & vA 68 & 4485 & K4 & & -3.92 & $<$ & -5.64 & $<$ & 3.0 & \pm 0.4 & $\checkmark$ & (9) & a) d) \\
\hline 04201057-1445400 & BD-15 767 & 4415 & K5 & $<$ & -4.82 & $<$ & -5.58 & $<$ & 3.0 & \pm 0.6 & & (9) & a) h) \\
\hline $03202921+0827161$ & G 79-28 & 4390 & K5 & & -4.39 & $<$ & -5.73 & $<$ & 3.0 & \pm 0.4 & & (9) & a) g) \\
\hline $04070122+1520062$ & Pels 15 & 4380 & K5 & & -4.35 & $<$ & -5.69 & & 3.0 & \pm 0.3 & & (9) & a) d) \\
\hline $04333716+2109030$ & vB 290 & 4330 & K5 & & -4.50 & $<$ & -5.68 & $<$ & 3.0 & \pm 0.5 & & $(9)$ & a) d) \\
\hline $04094935+0918197$ & HG 7-115 & 4315 & K5 & & -4.51 & $<$ & -5.76 & $<$ & 3.0 & \pm 0.4 & & (9) & a) d) \\
\hline $04052565+1926316$ & vB 226 & 4280 & K5 & & -4.34 & $<$ & -5.38 & & 4.3 & \pm 0.7 & & (9) & a) d) \\
\hline $04334192+1900504$ & vB 291 & 4205 & K6 & & -4.38 & $<$ & -5.64 & & 3.8 & \pm 0.4 & & (9) & a) d) \\
\hline $04140812+0924240$ & HG 7-129 & 4130 & K7 & $<$ & -4.23 & $<$ & -5.47 & $<$ & 3.0 & \pm 0.8 & & (9) & c) h) \\
\hline $04151038+1423544$ & vA 72 & 4085 & K7 & & -4.29 & $<$ & -5.43 & & 6.0 & \pm 1.0 & & (9) & a) d) \\
\hline
\end{tabular}


Table A.1.: Activity and rotation results for the Hyades combined catalog (continued).

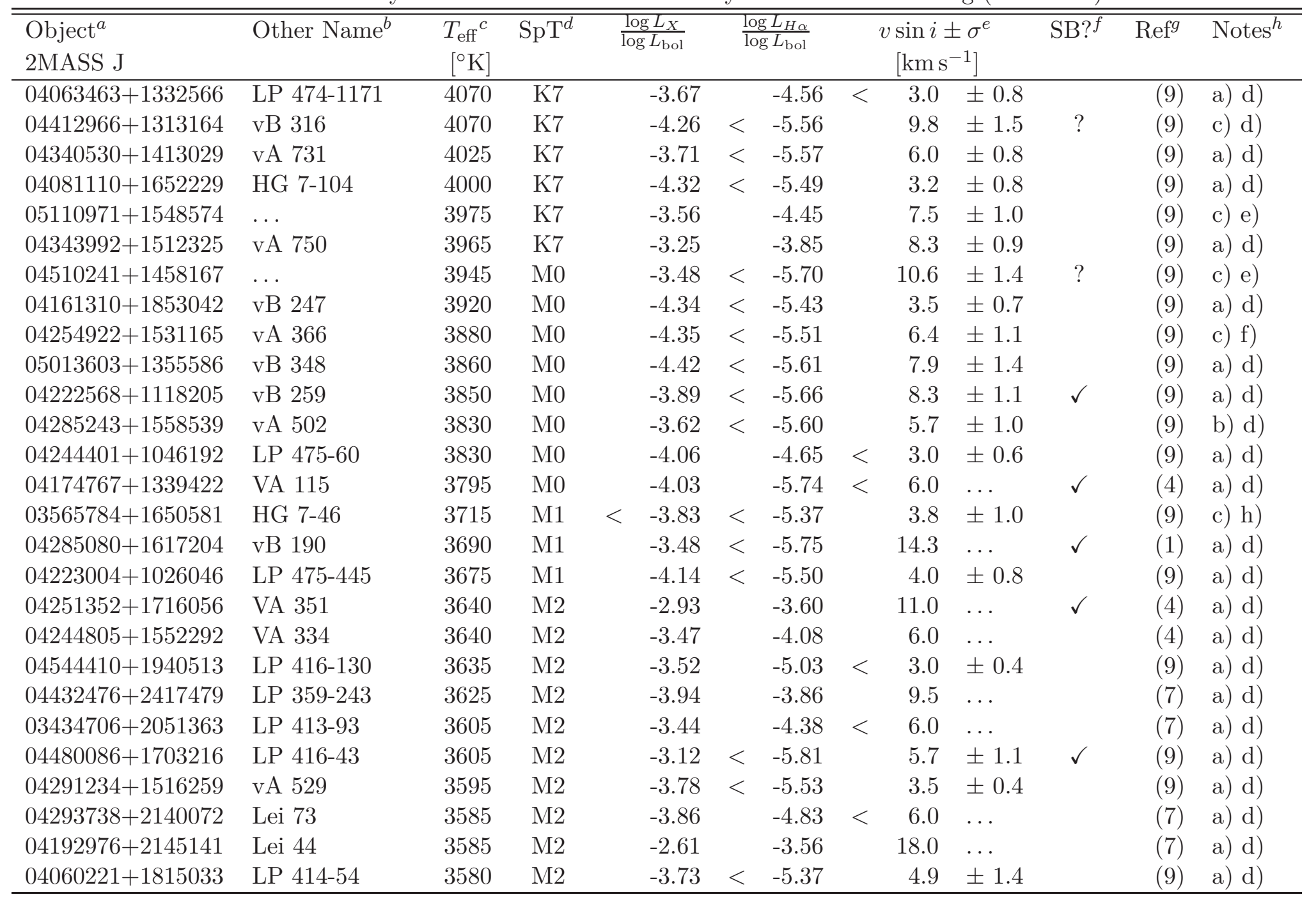


Table A.1.: Activity and rotation results for the Hyades combined catalog (continued).

\begin{tabular}{|c|c|c|c|c|c|c|c|c|c|c|c|c|c|}
\hline \multirow{2}{*}{$\begin{array}{l}\text { Object }^{a} \\
\text { 2MASS J } \\
04404249+0213522\end{array}$} & \multirow{2}{*}{$\begin{array}{l}\text { Other Name }{ }^{b} \\
\ldots .\end{array}$} & \multirow{2}{*}{$\begin{array}{l}T_{\mathrm{eff}^{c}} \\
{\left[{ }^{\circ} \mathrm{K}\right]}\end{array}$} & \multirow{2}{*}{$\begin{array}{c}\mathrm{SpT}^{d} \\
\mathrm{M} 2\end{array}$} & \multicolumn{2}{|c|}{$\frac{\log L_{X}}{\log L_{\mathrm{bol}}}$} & \multicolumn{2}{|c|}{$\frac{\log L_{H \alpha}}{\log L_{\mathrm{bol}}}$} & \multicolumn{3}{|c|}{$\begin{array}{c}v \sin i \pm \sigma^{e} \\
{\left[\mathrm{~km} \mathrm{~s}^{-1}\right]}\end{array}$} & \multirow[t]{2}{*}{$\mathrm{SB}^{f}{ }^{f}$} & \multirow{2}{*}{$\begin{array}{r}\operatorname{Ref}^{g} \\
(9)\end{array}$} & \multirow{2}{*}{$\begin{array}{l}\text { Notes }^{\prime} \\
\text { a) h) }\end{array}$} \\
\hline & & & & $<$ & -4.12 & $<$ & -5.37 & & 3.5 & \pm 0.8 & & & \\
\hline $03561242+1825003$ & GH 7-44 & 3560 & M2 & $<$ & -3.84 & & -4.87 & $<$ & 6.0 & $\ldots$ & & (4) & c) h) \\
\hline $04412876+1200337$ & LP 475-1699 & 3550 & M2 & & -3.63 & $<$ & -5.44 & & 3.5 & \pm 0.8 & & (9) & a) d) \\
\hline $04412780+1404340$ & $\ldots$ & 3550 & M2 & & -2.60 & & -3.47 & & 17.9 & \pm 1.0 & $?$ & (9) & a) e) \\
\hline $04165445+1621249$ & VA 96 & 3545 & M2 & $<$ & -3.81 & & -5.46 & $<$ & 6.0 & $\ldots$ & & (4) & a) d) \\
\hline $04122173+1615033$ & RHy 100 & 3545 & M2 & & -3.14 & & -4.31 & $<$ & 6.0 & $\ldots$ & $\checkmark$ & (7) & a) d) \\
\hline $04062097+2325274$ & LP 357-160 & 3540 & M2 & & -3.31 & & -4.55 & $<$ & 6.0 & $\ldots$ & & (7) & c) d) \\
\hline $04425093+1929137$ & LP 415-353 & 3535 & M2 & $<$ & -3.77 & & -5.13 & $<$ & 6.0 & $\ldots$ & & (4) & a) d) \\
\hline $04360416+1853189$ & LP 415-1582 & 3535 & M2 & & -2.75 & & -3.66 & & 18.4 & \pm 1.0 & $?$ & (9) & a) d) \\
\hline $04483062+1623187$ & LP 416-570 & 3535 & M2 & & -3.73 & $<$ & -5.61 & & 5.2 & \pm 1.2 & & (9) & a) d) \\
\hline $04591981+1023096$ & $\ldots$ & 3510 & M3 & $<$ & -3.43 & & -5.32 & & 3.6 & \pm 1.0 & & (9) & a) h) \\
\hline $04175499+1632401$ & RHy 142 & 3495 & M3 & & -2.94 & & -3.48 & & 11.5 & $\ldots$ & & (7) & b) d) \\
\hline $04211634+1125340$ & GH 7-173 & 3490 & M3 & $<$ & -3.32 & & -4.54 & $<$ & 6.0 & $\ldots$ & & (4) & c) d) \\
\hline $03523432+1115389$ & GH 7-26 & 3485 & M3 & $<$ & -4.14 & & -4.99 & $<$ & 6.0 & $\ldots$ & & (4) & c) d) \\
\hline $04350255+0839304$ & LP 415-292 & 3480 & M3 & & -2.47 & & -4.26 & & 11.4 & \pm 0.9 & & (9) & a) d) \\
\hline $04210061+2431080$ & LP 358-716 & 3475 & M3 & & -3.41 & & -3.81 & $<$ & 6.0 & $\ldots$ & & (7) & a) d) \\
\hline $04354850+1317169$ & RHy 350 & 3475 & M3 & & -2.89 & & -3.65 & $<$ & 6.0 & $\ldots$ & & $(7)$ & a) d) \\
\hline $04234294+1552513$ & RHy 189 & 3475 & M3 & & -3.26 & & -4.60 & $<$ & 6.0 & $\ldots$ & & $(7)$ & a) d) \\
\hline $04271663+1714305$ & vA 420 & 3475 & M3 & & -3.83 & & -4.75 & $<$ & 3.0 & \pm 0.8 & & (9) & a) d) \\
\hline $04341113+1133285$ & vB 294 & 3475 & M3 & & -2.78 & & -5.38 & & 7.2 & \pm 1.2 & $\checkmark$ & $(9)$ & a) d) \\
\hline $03583536+1720135$ & GH 7-58 & 3465 & M3 & $<$ & -3.60 & & -5.77 & $<$ & 6.0 & & & (4) & c) d) \\
\hline $04160357+1851330$ & RHy 123 & 3465 & M3 & & -3.56 & & -4.75 & $<$ & 6.0 & $\ldots$ & & $(7)$ & a) d) \\
\hline $04303385+1444532$ & LP 475-957 & 3465 & M3 & & -3.36 & & -4.84 & & 3.0 & \pm 1.9 & $\checkmark$ & (9) & a) d) \\
\hline $04401271+1917099$ & vB 310 & 3465 & M3 & & -3.34 & & -4.85 & $<$ & 3.0 & \pm 0.9 & & (9) & a) d) \\
\hline $04311955+1055256$ & RHy 301 & 3460 & M3 & $<$ & -3.44 & & -4.13 & & 12.0 & $\ldots$ & & (7) & a) h) \\
\hline $04311101+1623452$ & RHy 296 & 3455 & M3 & & -3.12 & & -3.79 & $<$ & 6.0 & $\ldots$ & & (7) & a) d) \\
\hline $04282878+1741453$ & vA 486 & 3450 & M3 & & -3.77 & & -3.99 & & 22.0 & $\ldots$ & $\checkmark$ & $(4)$ & a) d) \\
\hline
\end{tabular}


Table A.1.: Activity and rotation results for the Hyades combined catalog (continued).

\begin{tabular}{|c|c|c|c|c|c|c|c|c|c|c|c|c|c|}
\hline $\begin{array}{l}\text { Object }^{a} \\
\text { 2MASS J }\end{array}$ & Other Name ${ }^{b}$ & $\begin{array}{l}T_{\mathrm{eff}^{c}} \\
{\left[{ }^{\circ} \mathrm{K}\right]}\end{array}$ & $\mathrm{SpT}^{d}$ & & $\frac{\log L_{X}}{\log L_{\text {bol }}}$ & & $\frac{L_{H \alpha}}{\mathrm{g} L_{\mathrm{bol}}}$ & & $\begin{array}{r}v \sin i= \\
{[\mathrm{km} \mathrm{s}}\end{array}$ & & $\mathrm{SB} ?^{f}$ & $\operatorname{Ref}^{g}$ & Notes $^{h}$ \\
\hline $04223953+1816097$ & LP 415-27 & 3450 & M3 & & -3.46 & $<$ & -5.33 & $<$ & 3.0 & \pm 0.8 & & $(9)$ & a) d) \\
\hline $04275919+1845327$ & L72 & 3440 & M3 & & -3.60 & & -4.17 & $<$ & 6.0 & $\ldots$ & $\checkmark$ & (4) & a) d) \\
\hline $04172811+1454038$ & vA 106 & 3440 & M3 & & -3.00 & & -3.67 & & 10.0 & $\ldots$ & & (7) & a) d) \\
\hline $04322373+1745026$ & vA 673 & 3440 & M3 & & -3.17 & & -3.46 & & 23.6 & \pm 0.8 & $\checkmark$ & (9) & a) d) \\
\hline $04295572+1654506$ & vA 559 & 3440 & M3 & & -2.77 & & -4.43 & $<$ & 3.0 & \pm 0.7 & & (9) & a) d) \\
\hline $04332699+1302438$ & vA 709 & 3440 & M3 & & -3.15 & & -4.24 & $<$ & 3.0 & \pm 1.1 & $?$ & (9) & a) d) \\
\hline $04420453+1155137$ & LP $475-1747$ & 3440 & M3 & & -3.34 & $<$ & -5.51 & $<$ & 3.0 & \pm 0.8 & & (9) & a) d) \\
\hline $03591350+2025415$ & LP 414-479 & 3430 & M3 & & -3.29 & $<$ & -5.38 & $<$ & 3.0 & \pm 0.6 & & (9) & c) d) \\
\hline $04290015+1620467$ & RHy 269 & 3425 & M3 & & -2.83 & & -3.35 & & 26.0 & $\ldots$ & & (7) & a) d) \\
\hline $04251456+1858250$ & LP 415-794 & 3415 & M4 & & -2.75 & & -4.62 & & 5.3 & \pm 0.9 & & (9) & a) d) \\
\hline $04272407+1407072$ & RHy 242 & 3410 & M4 & & -3.71 & & -4.02 & & 6.0 & $\ldots$ & & $(7)$ & a) d) \\
\hline $04175061+1828307$ & vB 250 & 3410 & M4 & & -2.96 & & -4.01 & $<$ & 3.0 & \pm 0.6 & & (9) & a) d) \\
\hline $04235070+0912193$ & GH 7-192 & 3405 & M4 & & -3.04 & & -3.98 & $<$ & 6.0 & $\ldots$ & $\checkmark$ & (4) & a) d) \\
\hline $04423029+2027115$ & LP $415-345$ & 3405 & M4 & & -3.23 & & -3.95 & $<$ & 6.0 & $\ldots$ & $\checkmark$ & (4) & c) d) \\
\hline $03413689+2320374$ & LP 356-778 & 3400 & M4 & $<$ & -3.87 & & -4.95 & $<$ & 6.0 & $\ldots$ & & (4) & a) d) \\
\hline $04153746+1644272$ & VA 76 & 3395 & M4 & $<$ & -3.73 & & -5.75 & $<$ & 6.0 & $\ldots$ & & (4) & a) d) \\
\hline $04254182+1900477$ & LP 415-829 & 3395 & M4 & $<$ & -3.82 & & -6.17 & $<$ & 6.0 & $\ldots$ & & (4) & a) d) \\
\hline $04005947+1420454$ & RHy 28 & 3395 & M4 & & -3.16 & & -3.63 & & 11.5 & $\ldots$ & & $(7)$ & a) d) \\
\hline $04290099+1840254$ & vB 278 & 3395 & M4 & & -2.77 & & -3.77 & & 18.7 & \pm 0.5 & $\checkmark$ & (9) & a) d) \\
\hline $04572755+1356427$ & LP $476-648$ & 3385 & M4 & & -3.39 & & -4.00 & & 6.0 & $\ldots$ & & (7) & a) d) \\
\hline $04330500+2333180$ & LP 358-735 & 3385 & M4 & & -3.41 & & -3.87 & & 25.5 & $\ldots$ & & $(7)$ & a) d) \\
\hline $04161086+1132016$ & RHy 128 & 3375 & M4 & $<$ & -3.13 & & -4.20 & & 19.0 & $\ldots$ & & $(7)$ & a) h) \\
\hline $04124992+1114462$ & RHy 101 & 3375 & M4 & $<$ & -3.25 & & -4.48 & & 16.0 & $\ldots$ & & $(7)$ & a) h) \\
\hline $04282241+1349219$ & RHy 260 & 3370 & M4 & & -3.13 & & -4.19 & $<$ & 2.5 & $\ldots$ & & $(7)$ & a) d) \\
\hline $04235033+1455174$ & VA 288 & 3365 & M4 & & -3.13 & & -3.99 & & 7.0 & $\ldots$ & $\checkmark$ & (4) & a) d) \\
\hline $04251691+0804039$ & GH 7-206 & 3365 & M4 & & -3.62 & & -4.07 & $<$ & 6.0 & $\ldots$ & & (4) & a) d) \\
\hline $04211682+2311525$ & LP 358-717 & 3365 & M4 & & -3.03 & & -4.10 & & 18.0 & $\ldots$ & & $(7)$ & a) d) \\
\hline
\end{tabular}


Table A.1.: Activity and rotation results for the Hyades combined catalog (continued).

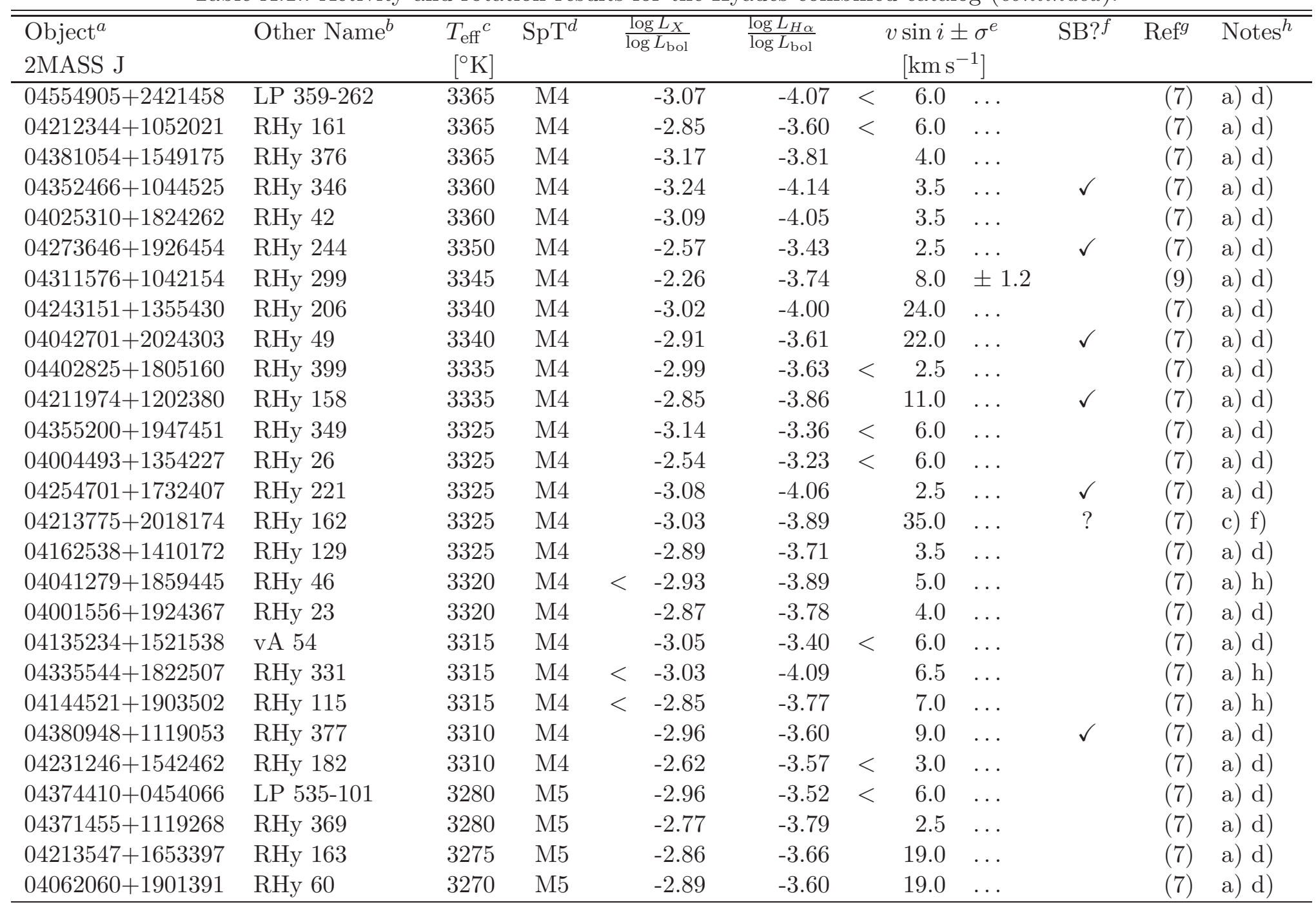


Table A.1.: Activity and rotation results for the Hyades combined catalog (continued).

\begin{tabular}{|c|c|c|c|c|c|c|c|c|c|c|c|c|}
\hline $\begin{array}{l}\text { Object }^{a} \\
\text { 2MASS J }\end{array}$ & Other Name ${ }^{b}$ & $\begin{array}{l}T_{\mathrm{eff}^{c}} \\
{\left[{ }^{\circ} \mathrm{K}\right]}\end{array}$ & $\mathrm{SpT}^{d}$ & \multicolumn{2}{|c|}{$\frac{\log L_{X}}{\log L_{\mathrm{bol}}}$} & $\frac{\log L_{H \alpha}}{\log L_{\mathrm{bol}}}$ & \multicolumn{3}{|c|}{$\begin{array}{c}v \sin i \pm \sigma^{e} \\
{\left[\mathrm{~km} \mathrm{~s}^{-1}\right]}\end{array}$} & $\mathrm{SB} ?^{f}$ & $\operatorname{Ref}^{g}$ & Notes $^{h}$ \\
\hline $04243060+1552542$ & RHy 203 & 3265 & M5 & & -3.06 & -4.37 & $<$ & 6.0 & $\ldots$ & & $(7)$ & a) d) \\
\hline $04373210+1645317$ & RHy 371 & 3265 & M5 & & -2.46 & -3.28 & & 14.0 & $\ldots$ & $\checkmark$ & $(7)$ & a) d) \\
\hline $04403300+1801422$ & RHy 402 & 3260 & M5 & $<$ & -2.43 & -3.81 & & 11.5 & $\ldots$ & & $(7)$ & a) h) \\
\hline $04255164+1330097$ & VA 371 & 3255 & M5 & $<$ & -3.94 & -5.16 & $<$ & 6.0 & $\ldots$ & & (4) & a) d) \\
\hline $04305731+1218136$ & RHy 294 & 3255 & M5 & & -2.26 & -3.03 & & 11.0 & $\ldots$ & & $(7)$ & a) d) \\
\hline $04492830+1916386$ & LP $416-573$ & 3245 & M5 & & -2.86 & -3.77 & & 6.0 & $\ldots$ & & (7) & a) d) \\
\hline $04242092+1910509$ & RHy 199 & 3245 & M5 & $<$ & -2.81 & -3.86 & & 16.0 & $\ldots$ & & $(7)$ & a) h) \\
\hline $04370989+1206200$ & RHy 367 & 3215 & M5 & $<$ & -2.72 & -3.55 & & 3.0 & $\ldots$ & & $(7)$ & a) h) \\
\hline $04243058+1859131$ & RHy 202 & 3215 & M5 & $<$ & -2.81 & -3.94 & & 2.5 & $\ldots$ & $\checkmark$ & $(7)$ & a) h) \\
\hline $04302395+1729591$ & RHy 283 & 3200 & M5 & & -2.61 & -2.93 & $<$ & 6.0 & $\ldots$ & & $(7)$ & a) d) \\
\hline $04270642+1625482$ & RHy 240B & 3200 & M5 & $<$ & -2.68 & -4.36 & & 14.5 & $\ldots$ & $\checkmark$ & $(7)$ & a) h) \\
\hline $04270642+1625482$ & RHy 240A & 3200 & M5 & $<$ & -2.68 & -4.16 & & 14.5 & $\ldots$ & $\checkmark$ & $(7)$ & a) h) \\
\hline $04311605+1212005$ & RHy 298 & 3195 & M5 & & -2.61 & -3.48 & & 6.0 & $\ldots$ & & $(7)$ & c) f) \\
\hline $04262170+1800009$ & RHy 231 & 3195 & M5 & & -2.64 & -3.19 & & 6.5 & $\ldots$ & & $(7)$ & a) d) \\
\hline $04070323+2016510$ & RHy 64 & 3195 & M5 & $<$ & -2.97 & -3.87 & & 19.0 & $\ldots$ & & $(7)$ & a) h) \\
\hline $04120760+1737341$ & RHy 99 & 3190 & M5 & & -2.33 & -3.09 & $<$ & 6.0 & $\ldots$ & $\checkmark$ & $(7)$ & a) d) \\
\hline $04311261+1228385$ & RHy 297 & 3190 & M5 & & -3.46 & -3.59 & & 13.0 & $\ldots$ & & $(7)$ & c) f) \\
\hline $04120334+2049527$ & RHy 98 & 3190 & M5 & & -2.91 & -3.73 & & 23.0 & $\ldots$ & & $(7)$ & a) d) \\
\hline $03583070+2715238$ & RHy 9 & 3190 & M5 & $<$ & -2.73 & -3.68 & & 3.5 & $\ldots$ & & $(7)$ & c) h) \\
\hline $04173224+1656591$ & RHy 138 & 3180 & M5 & $<$ & -2.69 & -4.21 & & 22.0 & $\ldots$ & & $(7)$ & a) h) \\
\hline $04095712+1622417$ & RHy 83 & 3165 & M5 & $<$ & -2.94 & -4.08 & & 8.0 & $\ldots$ & & $(7)$ & a) h) \\
\hline $03545319+1618563$ & GH 7-33 & 3160 & M5 & & -1.87 & -2.72 & & 12.5 & & $?$ & $(7)$ & a) d) \\
\hline $04394496+1805413$ & RHy 390 & 3160 & M5 & $<$ & -2.77 & -3.85 & & 18.0 & $\ldots$ & & $(7)$ & a) h) \\
\hline $04251652+1618086$ & RHy 219 & 3155 & M5 & & -3.12 & -3.46 & & 7.0 & $\ldots$ & & $(7)$ & a) d) \\
\hline $04161352+1647481$ & RHy 126 & 3150 & M5 & & -3.20 & -3.45 & & 21.0 & $\ldots$ & $?$ & (7) & c) f) \\
\hline $04382771+1600109$ & Br 804 & 3125 & M5 & $<$ & -2.63 & -4.07 & & 31.0 & $\ldots$ & & $(7)$ & c) h) \\
\hline $04180899+1724586$ & VA 127 & 3115 & M5 & $<$ & -2.62 & -4.09 & & 6.5 & $\ldots$ & & $(4)$ & a) d) \\
\hline
\end{tabular}


Table A.1.: Activity and rotation results for the Hyades combined catalog (continued).

\begin{tabular}{|c|c|c|c|c|c|c|c|c|c|c|}
\hline $\begin{array}{l}\text { Object }^{a} \\
\text { 2MASS J }\end{array}$ & Other Name ${ }^{b}$ & $\begin{array}{l}T_{\text {eff }}{ }^{c} \\
{\left[{ }^{\circ} \mathrm{K}\right]}\end{array}$ & $\mathrm{SpT}^{d}$ & & $\frac{\operatorname{og} L_{X}}{\operatorname{gg} L_{\mathrm{bol}}}$ & $\frac{\log L_{H \alpha}}{\log L_{\mathrm{bol}}}$ & $\begin{array}{c}v \sin i \pm \sigma^{e} \\
{\left[\mathrm{~km} \mathrm{~s}^{-1}\right]}\end{array}$ & $\mathrm{SB} ?^{f}$ & $\operatorname{Ref}^{g}$ & Notes $^{h}$ \\
\hline $04180899+1724586$ & RHy 143 & 3115 & M5 & & -2.39 & -3.39 & $4.0 \quad \ldots$ & & (7) & a) d) \\
\hline $04153255+2048274$ & RHy 119 & 3095 & M5 & & -2.05 & -3.76 & 7.5 & $\checkmark$ & (7) & c) d) \\
\hline 04395159+1939344 & RHy 391 & 3080 & M5 & & -2.80 & -3.21 & 24.0 & $?$ & (7) & c) f) \\
\hline $04300417+1604079$ & RHy 281 & 3055 & M5 & $<$ & -2.53 & -3.57 & 9.5 & $?$ & (7) & c) h) \\
\hline $04403699+1638088$ & RHy 403 & 3050 & M6 & $<$ & -2.53 & -3.64 & 5.5 & $\checkmark$ & (7) & c) h) \\
\hline $04385847+1539412$ & RHy 386 & 3020 & M6 & $<$ & -2.43 & -4.03 & 9.0 & & (7) & c) h) \\
\hline $04214435+2024105$ & RHy 165 & 2995 & M6 & $<$ & -2.43 & -3.94 & 17.0 & $?$ & (7) & c) h) \\
\hline $04261903+1703021$ & RHy 230 & 2940 & M6 & $<$ & -2.33 & -3.68 & 16.0 & & (7) & c) h) \\
\hline $04214955+1929086$ & Br 262 & 2885 & M6 & & -2.32 & -3.72 & 37.0 & $\checkmark$ & (7) & c) f) \\
\hline
\end{tabular}

Notes. (a) 2MASS identifiers. (b) Only the most common other identifier(s) are listed. For nomenclature and sources, see Table 3.2. (c) Effective

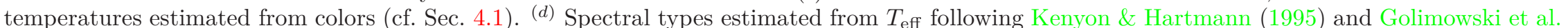
(2004). (e) Formal $1 \sigma$ errors available only for our new measurements, see Sec. 4.4.4. (f) Confirmed binaries (" $\checkmark ")$ or spectroscopic/candidate binaries ("?"). (g) For references for $\frac{\log L_{H \alpha}}{\left.\log L_{1}\right)}$ and $v \sin i$, see below. ( ${ }^{(h)}$ Notes: luminosities derived with distances from parallaxes in a) Röser et al. (2011), b) Hanson (1975) or Schwan (1991); c) fixed to 45 pc (cluster center).

X-ray (ROSAT) countrates from d) Stern et al. (1995), e) Voges et al. (1999), f) ROSAT Consortium (2000), g) Voges et al. (2000); h) upper limits for non-detections.

Notes on individual objects: In binaries, properties are measured for the system if not noted otherwise.

$2 \mathrm{M} \mathrm{J} 04270642+1625482 \mathrm{AB}\left(\mathrm{RHy} 240 \mathrm{~A}\right.$ and $\mathrm{B}$ ): $\mathrm{H} \alpha$ and $T_{\text {eff }}$ are estimated individually for $\mathrm{A}$ and $\mathrm{B}, L_{X}$ and $v \sin i$ are for the $\mathrm{AB}$ system. $T_{\text {eff }}$ derived from optical, not NIR photometry.

2M J04480086+1703216 (LP 416-43): averaged $v \sin i$ of the individual componenents, $\mathrm{H} \alpha$ for total system.

2M J04322373+1745026 (VA 673): secondary (white dwarf) has negligible effect on parameters.

2M J04412966+1313164 (vB 316): averaged $v \sin i$ of the individual componenents, $\mathrm{H} \alpha$ for total system.

References. (1) Griffin et al. (1985); (2) Radick et al. (1987); (3) Stauffer et al. (1987); (4) Stauffer et al. (1997a); (5) Bleach et al. (2002b); (6) Mermilliod et al. (2009); (7) Reid \& Mahoney (2000); (8) Seifahrt et al. (2009); (9) this work. 



\section{Part II.}

NAGICS-A novel absorption gas cell design for the CRIRES spectrograph 



\section{Abstract}

I present a design concept (NAGICS) for a long-path gas absorption cell for use with the CRIREs high-resolution near infra-red spectrograph facility at the VLT. This novel design overcomes the limitations of the current generation of short-path calibration gas cells in terms of attainable spectral fidelity, and provides access to gaseous calibrators in previously unsuitable wavelength domains.

The concept presented here, for the first time, introduces a purely reflective optical apparatus that enables an optical path-length of several meters through a captive gas, while retaining the spectrograph setup without any opto-mechanical changes.

I demonstrate the feasibility of the gas cell design by detailed modelling and a detailed performance analysis. The results show that in combination with CRIRES, the novel cell design is capable of delivering diffraction limited image quality to the spectrograph, while preserving all instrumental properties over the entire supported wavelength range $(1-5 \mu \mathrm{m})$, for a field of view that covers the entire entrance slit. Inevitable losses in throughput by the 14 additional optical elements (mirrors and windows) are kept at a minimum, with only minor impact on the overall efficiency.

Benchmark tests with ammonia and methane-13 as captive gases further demonstrate the potential of long optical absorption paths at low pressures, providing a much improved line depth, -sharpness, and -coverage over existing short pathlength cells. 



\section{Introduction}

\section{Contents}

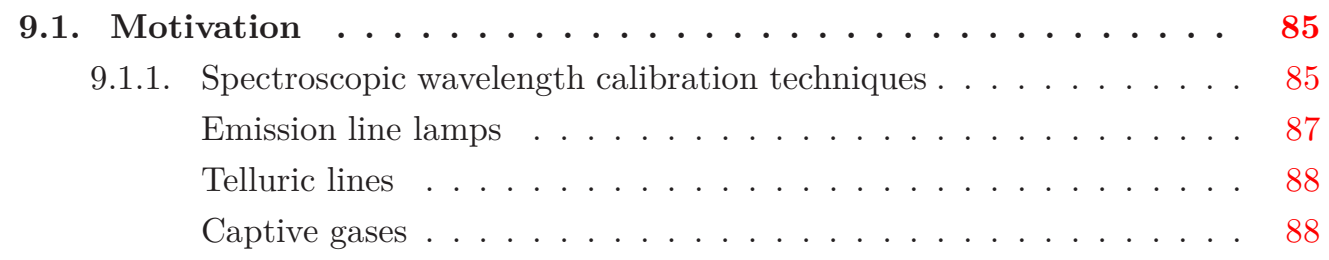

9.1.2. Why use a captive gas as a wavelength reference? . . . . . . . . . . 89

9.2. Scope of this work .................. 91

\subsection{Motivation}

The tremendous success in the field of exoplanetary research in the last decade or so has only been attainable with the major technological advances in recent years. The advent of large format, high-fidelity imaging sensor arrays has allowed for efficient photometric planet search programs, both ground-based and from space, that cover large enough areas of the sky to survey millions of targets simultaneously and continuously over a long period of time. Likewise, spectroscopic planet detection campains and characterization efforts rely on highprecision measurements (eg. radial velocities) only feasible with large detector mosaics, ie. a large wavelength coverage.

From the nearly 950 extra-solar planets confirmed to date ${ }^{1}$, the majority of them has been detected by means of radial-velocity (RV) monitoring of their host stars. The RV method is thus by far the most efficient planet finding (and confirmation) technique, and it is the only one that gives direct access to the planetary mass parameter. The underlying principle exploited with the RV technique is simple: determining the periodic Doppler-shift variation in the light of a planet hosting star, induced by the subtle gravitational pull of an orbiting (planetary) body. Yet, the measurement of the Doppler-shift variation for ever lower planetary masses raises extraordinary demands on the precision that must be met to disentangle the corresponding signal of the planet's reflex motion.

\subsubsection{Spectroscopic wavelength calibration techniques}

High-resolution spectroscopy demands for adequate wavelength references. Mapping the dispersed light in the focal plane of a spectrograph onto a scale of known wavelength positions is not a trivial task. It involes identification and comparison of a set of spectral lines (eg. Breckinridge et al. 1975), observed under the instantanous conditions, to the positions of the same set of lines in physical units in a defined reference frame ("wavelength solution"). Several complicating agents naturally render such an endeavour difficult:

\footnotetext{
${ }^{1}$ As of Sep 1st, 2013, 941 exoplanets have been detected (and confirmed) in 726 planetary systems, thereof 532 by means of RV monitoring.
} 

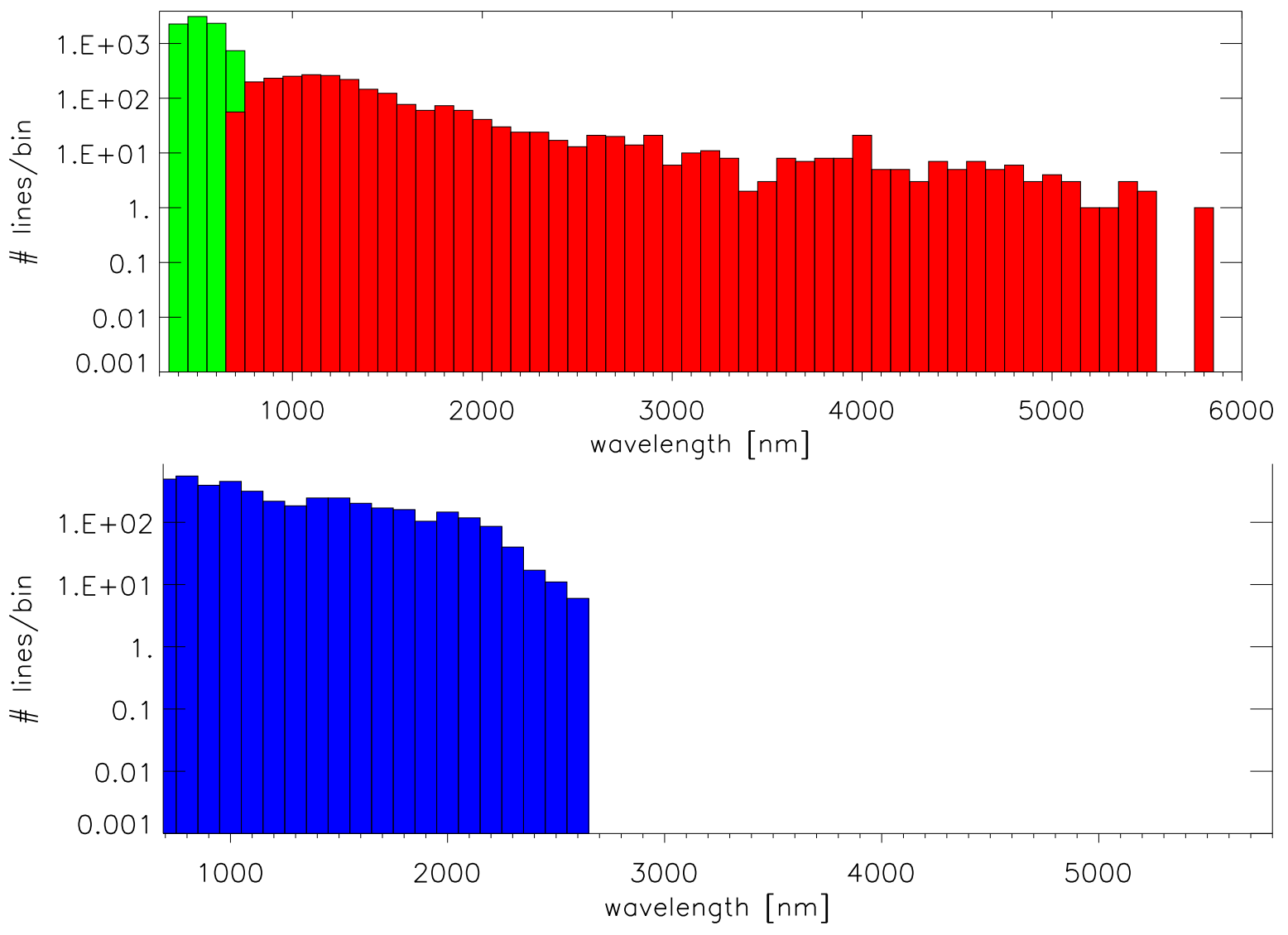

Figure 9.1.: Spectral line distribution of wavelength calibration sources. Top panel: line densities for ThAr HCL in the visible and near infra-red regions. Data from the atlas of Lovis \& Pepe (2007, green) and Kerber et al. (2008, red). Bottom panel: emission line densities for the telluric hydroxyl molecule (OH-airglow). Linelist based on the HITRAN database (Rothman et al. 2009). Note that the number of lines is given in log-scale, the bin size is $100 \mathrm{~nm}$.

- The larger the instrumental resolving power, the higher is the accuracy needed for the reference wavelength positions.

- The set of calibration lines should be equally spread in wavelength over the entire detector plane.

- A large number of deep, sharp, preferably unblended calibration lines is required over the entire instrumental wavelength range.

- Any changes of the environmental conditions over the course of the observation will affect the dispersion relation, and need to be accounted for to achieve the best precision.

- Any instabilities (instrumental, environmental,... ) between the time of recording of the calibration lines, and the time of the observation of the astronomical target, introduces uncertainties to the wavelength solution of the scientific observations.

A high degree of sophistication has been developed in the past decades to establish suitable sources of spectral reference lines in astronomical spectroscopy. In the traditionally well 


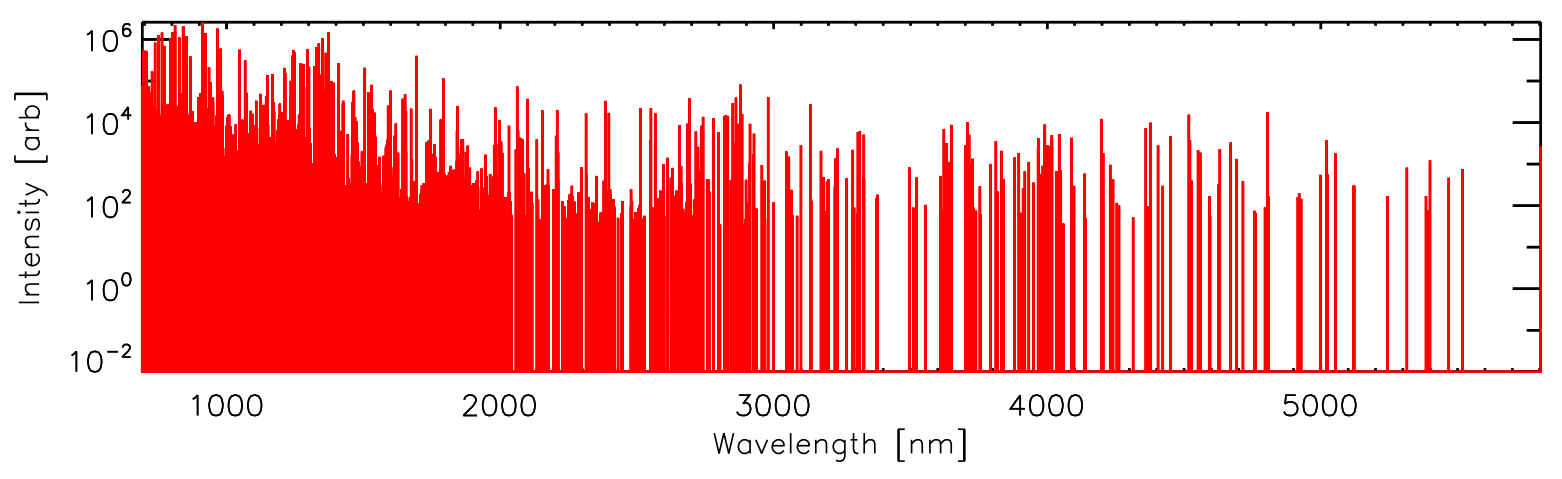

Figure 9.2.: Emission line spectrum of a ThAr HCL in the NIR domain. The emission line strength is largely inhomogneous (note the logscale).

explored regimes of ultra-violet to visible wavelengths, a variety of techniques are available and routinely used. The situation is different in spectral domains that have only recently become accessible to high-resolution spectrographs, such as the near infra-red (NIR, $1-5 \mu \mathrm{m})$. Recent advances in detector technology have opened this domain to efficient high-resolution work that has previously only been attainable in the visible. However, at these wavelengths atomic transitions become infrequent, so that sources of precision reference lines working in the visible are mostly not applicable in the NIR. Molecular rotation-vibrational transitions are omnipresent in the NIR, but many molecular compounds are lacking precise laboratory data. Means of precision wavelength calibration in the NIR are thus not on par with those in the visible regime.

A deeper understanding of the strengths and weaknesses of the different wavelength standards currently in use is indicative in order to appraise application scenarios based on the NAGICS approach. Therefore, I briefly summarize the most prominently used techniques of wavelength calibration for astronomical spectra in the following.

\section{Emission line lamps}

In the UV and optical wavelength range, astronomical wavelength calibration is usually performed using arc lamp spectra. Hollow-cathode discharge lamps (HCL) are accepted wavelength standards and commercially available. Thorium (Th) has proven to be a particularly suitable emission line source (Wybourne \& Meggers 1965), and is most often used for high resolving powers in ThAr or ThNe HCLs. Thorium provides a multitude of transitions from various ionization states that are accurately measured in the laboratory (Palmer \& Engleman 1983). There is only one stable isotope $\left({ }^{232} \mathrm{Th}\right)$, and the filling gas Argon has a high purity $\left({ }^{40} \mathrm{Ar}\right)$; both elements come with even numbers of nuclei, so that no isotopic nor hyperfine structure is seen (Rosman \& Taylor 1998). As a result, ThAr offers sharp, narrow, and symmetric spectral features with numerous lines and high line density (Murphy et al. 2007a) in the visible. It is particularly advantagous for high-resolution work, as many lines appear unresolved at resolving powers as high as $R \approx 150000$ (Balona 2010). As a result, ThAr is the default wavelength standard in astronomical spectroscopy.

However, there are downsides of HCLs with important implications for high-precision wavelength calibration. One of the major problems with eg. ThAr arc lamps is the presence of blended lines. Even at very high resolution, many lines appear blended, so that a careful selection of lines actually to be used as a reference in the wavelength calibration process is 
mandatory, for each given resolving power $R$ (at low $R$, HCLs with fewer spectral lines are beneficial, eg. $\mathrm{CuAr}$, as the number of available unblended lines drops). As the ThAr relative line intensities vary greatly (cf. Fig. 9.2), (unresolved) blended lines become assymetric and thus their wavelength position difficult to measure during the calibration process, introducing uncertainties when comparing such positions to the laboratory measurements acting as the reference (de Cuyper \& Hensberge 1998).

The individual line intensities also vary from lamp to lamp, and are a function of age, pressure, and operating current. These effects further degrade the stability as a reference source. ThAr does not exhibit a homogenous density of spectral features in terms of wavelength. While the line density is high in the optical, it significantly decreases for longer wavelengths (cf. Fig. 9.1). In the NIR, ThAr lines become more and more infrequent, which restrains the ability as a universal calibration source longwards of $1 \mu \mathrm{m}$, even more so for highresolution instruments with a limited instantanous wavelength coverage. Recently, Kerber et al. (2008) have presented extended laboratory ThAr line measurements that allow usage of this HCL-type for generic wavelength calibration up to $2.5 \mu \mathrm{m}$ (specifically for CRIRES, ie. at $R=10^{5}$, yielding a level of $50 \mathrm{~m} / \mathrm{s}$ precision for the line positions). Nevertheless, the ThAr line coverage and density is not sufficient for precision calibration in larger regions of the NIR (Mickelson et al. 2001), compared to the visible range with a ThAr line position accuracy of $16-82 \mathrm{~m} / \mathrm{s}$ (Palmer \& Engleman 1983) and more recently $\approx 10 \mathrm{~m} / \mathrm{s}$ (Lovis \& Pepe 2007, in the $400-700 \mathrm{~nm}$ range). Fig. 9.1 illustrates the ThAr line densities across the UV to the NIR wavelength range, while Fig. 9.2 emphasizes the vastly different line strengths.

\section{Telluric lines}

Astronomical wavelength calibration in the (near) infra-red domain typically relies on spectral features imprinted onto observations of the night-sky by earth's atmosphere. The telluric hydroxyl molecule $(\mathrm{OH})$ provides a large number of rotation-vibration transitions throughout the NIR (Rousselot et al. 2000). Emission lines from this nighttime OH-airglow are traditionally used for wavelength calibration, aided by laboratory reference data of $\mathrm{OH}$ (Abrams et al. 1994). As the sky features are simultaneously recorded with the science target, the approach is particularly useful.

The line density at high resolving powers, however, is too low and the lines too weak in a given wavelength range (Kerber et al. 2009) to allow accurate calibration. Fig. 9.1 compares the $\mathrm{OH}$ line density to that of ThAr HCLs.

Other telluric molecules such as $\mathrm{CO}_{2}$ (in the NIR) or $\mathrm{O}_{2}$ (in the visible) are potentially suitable calibrators in specific wavelength intervals, yielding radial velocity precisions of $5-$ $10 \mathrm{~m} / \mathrm{s}$ (Figueira et al. 2010b,a). The presence and strength of telluric calibrators, however, are strongly site-dependent and prone to variations on different timescales from hours to years. Thus, a detailed understanding and modelling of the atmospheric conditions, based on simultaneously measured sounding data, is required, as eg. high-altitude winds induce significant uncertainties on the line positions ( $\leq 25 \mathrm{~m} / \mathrm{s}$, Kerber et al. 2009).

\section{Captive gases}

The benefits of the wavelength calibration technique using telluric features can be mimicked by exploiting ro-vibration lines from a selected molecular gas. This gas (or a mixture of several) is then kept under well controlled conditions (inside a gas cell vessel), and this vessel is brought into the light entering the spectrograph at the time of observations. The 


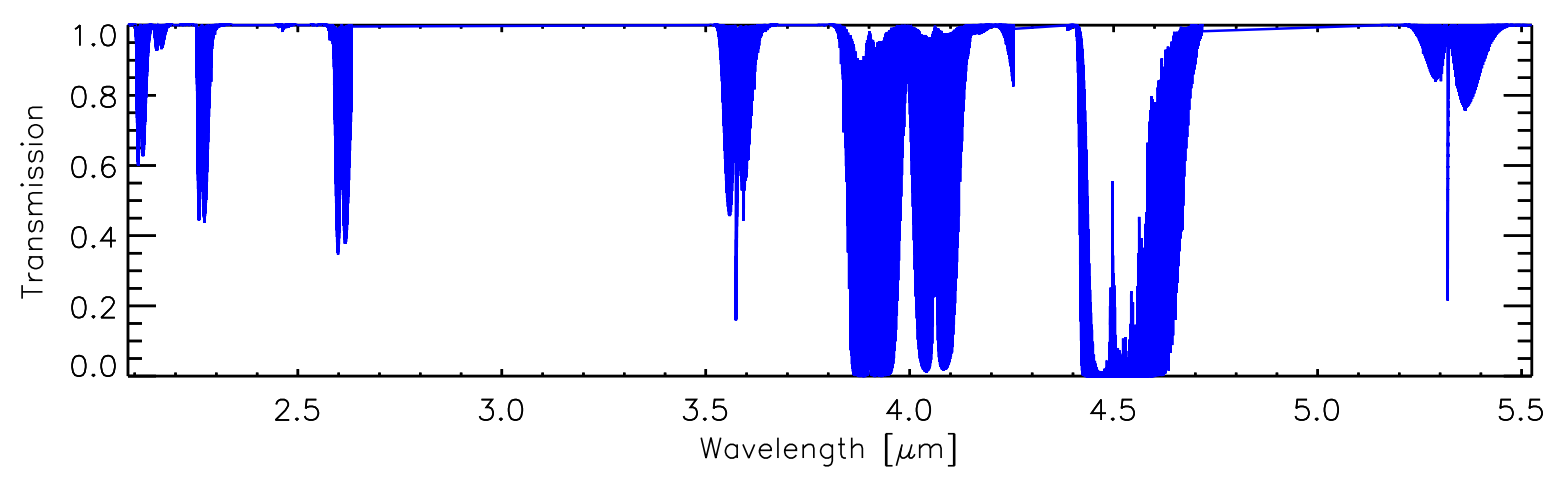

Figure 9.3.: Absorption lines of the wavelength standard $\mathrm{N}_{2} \mathrm{O}$ in the NIR. The wavelength coverage is bound to the transition bands, with vastly different line depths. Data from the CRIREs $\mathrm{N}_{2} \mathrm{O}$-calibration cell (Käufl et al. 2004).

effect is thus similar to the imprint of molecular features from the atmosphere, on the actual target spectrum itself, but for selected species and without the influences of the perturbing dynamical processes in the atmosphere.

By using a reference gas in the optical path shared with the light coming from the astronomical target, and possibly doing so in one step at the same time, no special calibration exposures are needed besides a detailed knowledge of the reference gas spectrum and its laboratory line positions. The gaseous absorption lines can thus be used to track all instrumental effects and uncertainties that follow in the optical chain. It is hence advisable to insert such a captive gas as early as possible into the beam, so that the calibration lines be imprinted before the light enters the spectrograph and other optical elements.

On the other hand, a major challenge is to identify a suitable gas that provides the necessary line density over the desired, broad spectral range (Fig. 9.3). Athmospheric constituents and molecular compounds present in the target of interest dramatically limit the choice of feasible gases, as those spectral features are difficult to disentangle from the same calibration features.

Chapter 10 discusses the benefits and challenges of absorption gas cells in more detail.

Other techniques for astronomical wavelength calibration purposes have emerged over the past years, notably Laser Frequency Combs (LFC) and Fabry-Pérot interferometers (FP). The former pose an ideal calibration source, as it covers the entire spectral range of interest with equally spaced, isolated lines. The line positions are known to very high precision, and all lines are of comparable intensity. Thus, LFCs are promising calibration sources for the next generation of extremely large telescopes (Steinmetz et al. 2008; Murphy et al. 2007b), as their anticipated precision will be limited by the calibration sources instead of by photon noise. Considerable effort is being made towards LFC turn-key solutions (Phillips et al. 2012; Doerr et al. 2012; Murphy et al. 2012), but other precision calibration methods remain the preferred choice for reasons of cost, proven long-term stability, and ease of operation and reliability at astronomical observatories (Osterman et al. 2007; Braje et al. 2008; Quinlan et al. 2010; Lo Curto et al. 2012).

\subsubsection{Why use a captive gas as a wavelength reference?}

The spectral fingerprint of a specific molecular gas in the optical path, as outlined above, may serve as a high-precision calibration source to reference the wavelength positions in ob- 
served stellar spectra to those line positions previously obtained for the gas under laboratory conditions. This approach yields high-precision velocimetry in the visible spectral windows (cf. Chapter 10), and is particularly promising to become a standard precision calibration technique also for the recently growing number of high-resolution spectrographs operating in the NIR, where universal calibration sources over wide spectral ranges (as ThAr in the visible) are not available.

In this section, I detail the general requirements that must be met and the challenges to be faced if a reference gas is projected to be a viable calibration source. This is to set the stage for a more detailed discussion on the actual design of a captive gas vessel and it's implementation in the following chapters.

In the following, I refer to any such device that enables to host a reference calibration gas in the optical path as an "emission or absorption gas cell".

Generally, two modes of operation of a gas cell are feasible.

1. Analogous to the ThAr HCL method, the calibration gas can be fed by a separate optical path or fiber, so that strictly speaking the reference spectrum is not imprinted onto the stellar spectrum, but is displaced on the detector, forming an independent spectral image. For practical reasons, this mode implies that the spectrograph be fiberfed, with a second calibration fiber reserved for the light coming from the gas cell. A suitable illumination of the reference gas is then required for the absorption lines to form.

2. The classical mode implies that the calibration gas is hosted in the exact same optical path as traversed by the light coming from the astronomical target. The reference gas is then illuminated by the stellar light, and no additional light source is needed. The spectral imprint from the gas is superimposed onto the stellar spectrum on the detector, and so cannot be separated (unless the cell is taken out of the optical chain). This strategy provides the maximum attainable precision, as any instrumental effects or instabilities ocurring to the optical path after the reference line formation will be experienced in the very same way by both the stellar spectral features and the calibration features. This mode of operation is applicable to virtually any telescope/spectrograph system, and does not imply a fiber-fed instrument. It is hence particularly interesting for existing multi-purpose facilities, since it allows an in-situ tracking of, in principle, any stability deficiencies of the instrument.

I will refer to the second mode as the default mode of gas cell operation throughout this work. For the high demands in precision for low-mass planetary RV work, this latter approach has a major advantage over other calibration techniques: high-precision wavelength calibration is feasible with modest instrumental stability. Other than the HCL calibration methods, which for highest precision require a very high degree of intrinsic stability (eg. HARPs; Mayor et al. 2003), by means of multi-stage ambient control and encapsulated vacuum housing of the spectrograph and the calibration unit; a calibration gas cell sets much lower demands on instrument stability (provided that the gas cell is stable).

A gas cell calibration approach thus can pave the way for high-precision applications on spectrographs not designed for the highest stability in the first place: while observing, the reference gas spectral features track the behaviour of all optical elements plus the detector under the instantanous conditions of illumination and environment. 


\subsection{Scope of this work}

Part two of the present thesis presents the conceptual design of a novel wavelength calibration cell to be deployed in the CRIRES spectrograph at ESO's Very Large Telescope (VLT). My approach is taking the existing gas absorption cell technology one step further by introducing an optical setup that permits path lengths through the captive gas an order of magnitude larger than existing calibration cells. With path lengths of up to several meters, compared to less than $20 \mathrm{~cm}$ path lengths in currently available cells, the present design enables an enhanced flexibility to allocate the desired gas properties, and empowers wavelength calibration with reference gases in spectral domains and with precision previously not accessible.

Present day gas absorption cells are comprised of tube vessels, containing the captive gas, which are placed into the optical train in front of a spectrograph. The devices are optically passive, so to not alter the telescope beam properties when inserted or taken out of the instrument, and hence have to mechanically fit into the available space between the telescope and the spectrograph. This causes a number of limitations (further detailed in Chapter 10) that hamper the use of such a device over a broad range of wavelengths, a characteristic that is highly desired for a versatile wavelength calibration source.

The work presented here overcomes the shortcomings of a "short" calibration cell that is to be fit into the very limited available space at most existing facilities. Instead of an optically passive container, I develop a cell design with active optical components, yet without disturbing the telescope beam properties entering the spectrograph and its pre-optics. The proposed device fits into the available tight space foreseen for a conventional calibration gas cell, and so does not require any alterations to existing the optical layout of the telescopespectrograph system.

At the same time, however, the path length that the telescope beam is traversing through the captive gas, is increased to a multiple of that offered by a conventional cell design. The much enhanced path length facilitates the use of reference calibration gases that otherwise, over short path lengths, do not provide spectral line systems with appropriate or advantagous properties for wavelength calibration purposes. The device thus acts in the same overall fashion as a conventional cell, as it can be inserted into and removed from the optical train without introducing any changes to the spectrograph's input beam properties.

The reference wavelength gas cell design conceptualized here is specifically tailored to the needs and conditions of the CRIRES spectrograph, mounted on one Nasmyth focus of Antu (UT1) at the VLT. A particular strength of the design approach is that it allows an easy adaptation to other facilities. The design is most useful to implement at existing facilities with high-resolution spectrographs that do offer only limited physical space to house calibration cells. Such instruments are not designed to host captive gas vessels with long optical paths in the first place. Wavelength calibration at potentially any of these instruments can benefit from an implementation of the proposed reference cell concept, opening a pathway to alleviate imperfections in the spectrograph's dispersion solution and, more importantly, its stability due to dynamical instrumental effects - a pre-requisite for eg. precision radial-velocity work.

A further design strength is the scalability of the optical path length. Following the same common methodology, path lengths of several meters can be realized. This degree of freedom allows to optimize wavelength calibration capabilities for the desired wavelength range, as it enables the use of gases and mixtures of gases with eg. low absorption coefficients, that would otherwise exhibit only shallow spectral lines or demand for impractical conditioning (pressure, temperature). The NAGICS design thus facilitates crafting a gas combination with appropriate parameters that is suitable for the demands and requirements of calibration in a 
wide range of wavelengths.

In this part of the thesis, I first develop the ideas that lead to the concept of NAGICS. I begin with a brief review of the conventional gas cells presently in use, and outline their drawbacks (Chapter 10). Efforts are then presented to circumvent the physical space limitations imposed by the CRIRES spectrograph. Next, I highlight the characteristics of the NAGICS strategy in Chapter 11, and describe the optical design approach and the requirements that must be met for CRIRES. A specific baseline design for CRIRES is then worked out and modelled in detail. This design is then subject to a detailed analysis of its optical performance, as well as to benchmark tests concerning the resulting gas transmission spectra for two selected species in Chapter 12. Also, a trade-off is discussed in order to simplify the realization of the proposed design, and estimates are derived for the performance with manufacturing/integration tolerance margins (Chapter 13). Thereafter, adaptions to other facilities are discussed before the present part is summarized in Chapter 14. 


\title{
10. Conventional absorption cell design
}

\author{
Contents

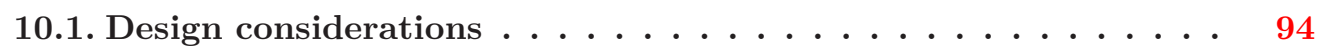

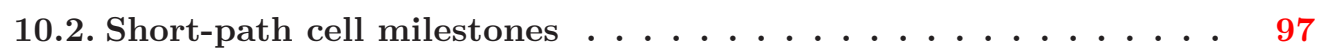 \\ 10.2.1. At optical wavelengths . . . . . . . . . . . . . . . 97

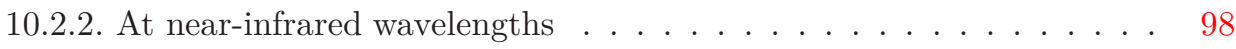 \\ 10.3. Shortcomings . . . . . . . . . . . . . . . 101 \\ 10.3.1. Why White- or Herriott cells do not work . . . . . . . . . . . . 102
}

The use of a captive gas as an astronomical wavelength reference dates back to Beckers (1976). In a study of sunspot motions, absorption lines from gaseous iodine $\left(\mathrm{I}_{2}\right)$ were employed as a stable wavelength reference. Koch \& Woehl (1984) further refined the technique to obtain solar Doppler shifts, which eventually became the "iodine method" later used extensively for precision RV measurements (eg. Marcy \& Butler 1992; Butler et al. 1996) for the purpose of exoplanet searches.

However, pioneering work in the field has been accomplished by Campbell \& Walker (1979), who conducted RV measurements with hydrogen fluoride (HF) as a means of reference wavelength calibration. The experiment proved that precision stellar radial velocities could be achieved with a captive gas placed into the optical path of the spectrograph, leaving reference absorption lines from the gas imprinted onto the stellar light during the observation. Campbell \& Walker were able to achieve an improvement of two orders of magnitude in RV precision, compared to what was attainable with photographic plate technology. The effort resulted in a 12 year long program (1980-1992) of monitoring 29 solar-like stars (including six giants) for RV variations, using a revised version of the HF absorption cell (Campbell et al. 1988). With a precision of $\approx 10 \mathrm{~m} / \mathrm{s}$, they had the capabilities at hands to detect Jovian-mass substellar companions. In fact, among their program stars were three targets that later were found to host extra-solar planets ( $\gamma$ Cep, $\beta$ Gem, $\epsilon$ Eri). Campbell et al. (1988) identified a "probable third body variation of $25 \mathrm{~m} / \mathrm{s}$ amplitude, $2.7 \mathrm{yr}$ period", corresponding to a companion mass of $1.7 M_{\mathrm{J}}$ from their first six year's data. However, it was not before 1995 until the very first exoplanet detection was explicitly claimed (and unambiguously detected) 51 Peg b. The Jovian planet around $\gamma$ Cep (HD 222404, K1 IV) remained putative (Walker et al. 1992, 1995) until finally confirmed by Hatzes et al. (2003). The signature of $\beta$ Gem (HD 62509, K0 IIIb) was firmly interpreted ${ }^{1}$ as a planetary companion later by Hatzes et al. (2006). The "probable variable" $\epsilon$ Eri (HD 22049, K2 V), quoted in Campbell et al. (1988) with a companion mass of $1.2-4.7 M_{\mathrm{J}}$, was established as a planet host from extended data also by the Texas group (Hatzes et al. 2000), with a tantalizing planetary period of $6.9 \mathrm{yr}$ $\left(0.9 M_{\mathrm{J}}\right)$.

With the milestone discovery of 51 Pegasi b (Mayor \& Queloz 1995), by means of Doppler monitoring (with a short period of $4.2 \mathrm{~d}$ as opposed to several years above) without a gas

\footnotetext{
${ }^{1}$ A possible $2.9 M_{\mathrm{J}}$ companion was already reported by Cochran \& Hatzes (1993).
} 
cell as wavelength reference, but instead with a highly stabilized spectrograph, two branches of exoplanet detection techniques exploiting the planetary reflex motion have emerged, both approaches being very successful.

Captive gases as a source of reference spectral lines for wavelength calibration offer a number of advantagous applications when employed at astronomical facilities. The gases can be selected and their properties tuned, in principle, to provide absorption or emission lines at precisely known positions in any particular wavelength region of interest.

Specifically at near infra-red wavelengths, other means of calibrating a spectrograph's dispersion relation are often sparse or not available at all. Thorium-Argon emission lamps, as an example, are frequently used at optical wavelengths where ThAr shows a rich and dense spectrum of atomic emission lines. However, ThAr lines become infrequent and less dense in the infra-red domain (Lovis \& Pepe 2007; Kerber et al. 2008). Other types of emission lamps are being considered, eg. Uranium-Neon (Redman et al. 2012), but suffer from incomplete or imprecise linelists or technical difficulties.

\subsection{Design considerations}

For high-precision wavelength calibration such as needed for RV planet detections on a few $\mathrm{m} \mathrm{s}^{-1}$ level, a gascell calibration source must meet the following conditions:

First, the gas should provide absorption bands in a wide wavelength region of interest. Ideally, it covers the entire instrumental range of the spectrograph (albeit this is not feasible for most cross-dispersed echelle instruments with large wavelength coverage). The lines should be deep and sharp at the given resolution, so that their line centers can be determined precisely. For the given spectral resolving power, blends must be avoided; so the line spacing should match (in other words, the spectrograph's resolution must not fall short of some minimum power). A high line density is desirable throughout the covered wavelength range, as this crucially determines the ability to precisely find a dispersion solution for the entire detector area, for any spectral region instantanously covered by a particular instrument setup. The gas absorption lines must be known to a high degree of precision, as any uncertainty in their positions immediately translates into a degraded achievable calibration precision. For this purpose, exquisit molecular line data is required to reference the actually measured gas line positions at the time of the stellar observation, and under the instantanous environmental and instrumental conditions. Alternatively, a measured ultra high-resolution spectrum of the gas under controlled conditions (ie. the entire gas cell under conditions of operation) may serve as the reference for the gas absorption lines (eg. with a FTS with $R \approx 10^{6}$ ). Most molecular ro-vibrational transition lines are not resolved even at very high $R$ (as an example, molecular iodine lines are all blended still at $R=1 \times 10^{5}$ ), which underscores the need for accurate line positions.

The fact that molecular transitions expose localized line systems (other than emission lamps such as ThAr) already makes clear that gaseous absorption calibrators are generally not easily suitable for very broadband applications as are HCLs. Also, the selection of a suitable gas ultimately depends on the characteristics of the spectrograph in question, most importantly its resolving power and spectral coverage.

Second, the properties discussed above must match the stellar targets of interest. This is particularly important for exoplanet RV applications. The wavelength window covered by the gas cell should be chosen so that the stellar spectrum in this region also exhibits numerous lines suitable for RV measurements. This (usable) RV information content ("Q-factor", Bouchy 
et al. 2001) is determined, among others, by the spectral richness of the wavelength region of interest, the line sharpness, and the achievable SNR. It thus naturally depends on the spectral type of the star.

At optical wavelengths, solar-type MS stars harbor a large RV information content, owing to numerous unblended, mostly atomic lines that can be exploited. The picture changes towards very low-mass stars (M-type), as their shifted SED renders the optical regime undetectable, and simply too faint even for the most nearby stars.

Instead, for M-stars the flux distribution peaks longwards of $1 \mu \mathrm{m}$, so that the NIR promises the best achievable SNR. A plentiful of stellar molecular lines at these wavelengths provides a high RV information content. Accordingly, for high-precision RV measurements, the target stars must match in wavelength to the reference gas cell employed (and vice versa).

Third, the wavelength range of operation of the calibration gas cell should be as free of telluric contaminations as possible. Telluric lines imposed onto the stellar spectrum due to the atmospheric composition above the observatory will degrade the attainable RV precision, as such lines will blend the stellar lines, thus compromising their RV quality. Additionally, the telluric contribution is prone to wavelength shifts and varies on different timescales. Taking into account the presence of tellurics adds an additional layer of complexity to the wavelength calibration process and to the determination of RVs, moreover as their occurence and strength is also site- and weather dependent.

Calibrating the telluric imprint by means of telluric standard star observations is deemed unfeasible for RV precisions of few $\mathrm{m} / \mathrm{s}$, required for low-mass planet detections. The offset in time, path through the atmosphere, and instrumental illumination inevitably imposed by such telluric observations precisely counteracts the principle of simultaneous calibration with a gas cell. However, sophisticated modelling of the inherent telluric spectrum seems a viable but challenging solution (Bean et al. 2010).

The issue of telluric contamination is particularly delicate in the NIR, where absorption lines of atmospheric constituents are almost omnipresent and thus unavoidable in any but the smallest wavelength windows. This poses a limiting factor for precision RV in the NIR (Seifahrt et al. 2010; Bean et al. 2010). An additional limitation is set by the prevailing species in the atmosphere. These species are mostly excluded as candidates for the reference gas, since the line systems from the gas cell and the telluric ones are generally not shifted enough to be separated. Disentangling both overlapping sources would hence be severely hampered.

Other concerns to be taken into account are related to the physical properties of the captive gas: The gas must be long-term stable, both chemically and with respect to temperature. It must also persist in a gaseous state at temperatures typically found at the observatory. Heating the gas cell is feasible $\left(50-100^{\circ} \mathrm{C}\right.$ in the case of $\mathrm{HF}$ and $\mathrm{I}_{2}$; see Sec. 10.2), but complicates operations and is highly undesirable for applications in the infrared, where the gas cell then increases the thermal background. If the absorption spectrum of the gas is prone to temperature shifts, thermal stabilization is mandatory to a degree where wavelength shifts over the expected range of temperatures can be considered negligible. Pressure stability is usually of little concern if the gas vessel is properly sealed. However, long-term seals stable over many years can be challenging to manufacture.

Practical considerations also arise for the applicability of a reference gas cell: the employed gas in use must not be corrosive to the vessel material (cf. HF above). Gaseous species that are hazardous, toxic, or lethal require special attention and precautions when operated at astronomical observatories, if at all. Although the volumes (and pressures) are low for existing gas cells, leakage or breakage at remote observing sites cannot be excluded and consequences 
need to be evaluated accordingly. The discussed considerations render the identification and selection of a suitable reference gas a challenging task.

So far, I have only discussed constraints imposed by or related to the calibrator. Additional instrumental boundary conditions for the gas cell vessel apply. I will revisit such issues in Sec. 10.2. For now, some important free parameters for designing a suitable gas can be identified. These parameters shape the transmission absorption spectrum, and so determine the achievable wavelength calibration precision. This, in turn, sets a major constraint on the achievable RV measurement precision (together with other factors such as the stellar spectrum, the instrument performance, etc.; see above). The parameters concerning the gas cell can be optimized for a specific wavelength/instrument/gas cell combination as outlined above, namely:

- The gas species

- Gas temperature

- Gas pressure

- Optical path length through the gas

- Resolving power of the spectrograph

The type of gas is usually governed by the general choice of wavelength range under consideration. It should be noted that isotopologues ${ }^{2}$ of a particular gas can considerably enhance the wavelength coverage of molecular absorption features. Changing the isotopic composition of a molecule results in wavelength shifts of the spectral features. It may also lead to additional (or reduced) degrees of freedom for ro-vibrational transitions, corresponding to the presence of additional spectral features at other wavelengths, compared to the normal, prevalent isotopologue of a given molecule. Thus, isotopologues are a very promising tool to adjust the line density and location; however, line lists for isotopologues are not readily available but for a few of the more common gases, and so experimental data is mostly needed before the performance of isotopologues can be assessed.

Such laboratory data can only be obtained by FTS measurements, provided that procurement and purity of an isotopologue is not a limitation. For example, Anglada-Escudé et al. (2012) conducted comparison measurements of methane isotopologues, finding that ${ }^{12} \mathrm{CH}_{3} \mathrm{D}$ is superior in terms of line density and spectral coverage in the $\mathrm{H}$ and $\mathrm{K}$ bands, over normal ${ }^{12} \mathrm{CH}_{4}$ (see Sec. 10.2.2).

As far as the parameters gas temperature and pressure are concerned, line broadening by these agents is to be kept at a minimum. The operating temperature should thus be kept at the ambient temperature level of the telescope or dome, and possibly stabilized. Pressure remains as an adjustable free parameter, and can be tuned to find the optimum depth of the gas absorption lines in question.

However, increasing the pressure also increases the intrinsic line width, counteracting the achievable calibration precision. As the spectrograph resolution is commonly set by existing instruments, or driven by other constraints, a compromise needs to be found to satisfy the need of calibration precision. The path length of the gas cell thus serves as a powerful means to keep the gas pressure low.

A long optical path through the captive gas provides deep absorption lines at the benefit of sharper lines. The downside is that for most spectrographs, unless it is specifically designed

\footnotetext{
${ }^{2}$ i.e. the same molecular structure, but where atoms have been replaced by different isotopes.
} 

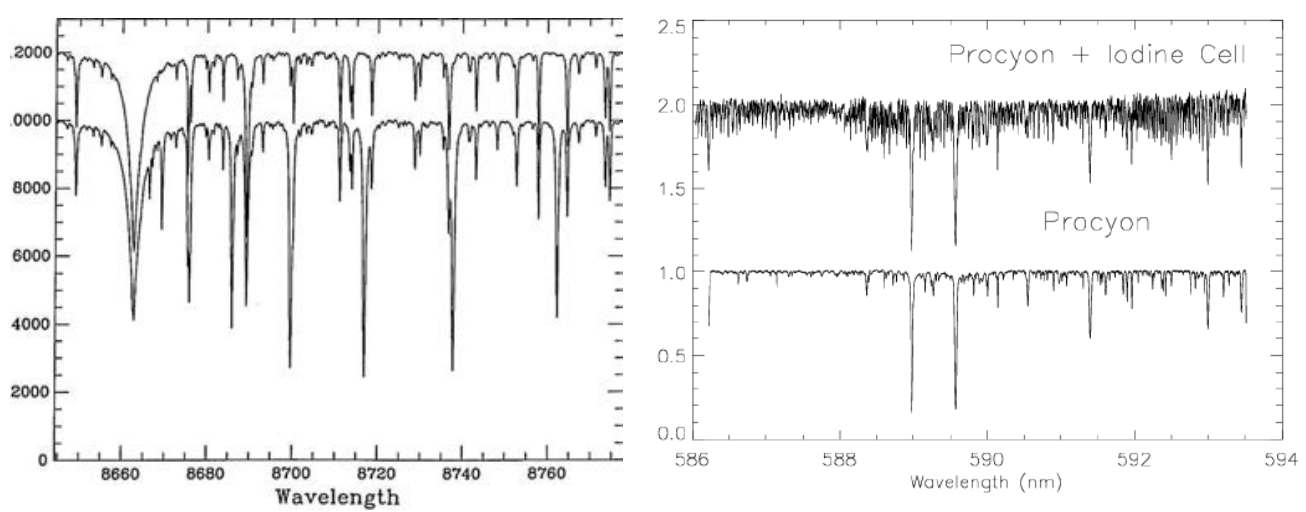

Figure 10.1.: Example of stellar spectra with imprints of HF (left panel, the lower spectrum is composed of the stellar and the HF contribution) and $\mathrm{I}_{2}$ (right panel). Reproduced from Campbell et al. (1988) and Claudi et al. (2005), respectively.

for this purpose (eg. GIANO/TNG; D'Amato et al. 2008), the available physical space to accomodate a calibration gas cell is very limited (eg. $\sim 10 \mathrm{~cm}$ in the case of CsHELL/IRTF (Anglada-Escudé et al. 2012); 20 cm for CRIREs/Vlt, cf. Sec. 11.2). The short space defines the maximum path length through the gas for conventional (single pass) cells, and hence poses a major limitation on the choice of gas and the necessary pressure to obtain deep yet sharp absorption features.

Chap. 11 is devoted to this issue and discusses how to mitigate path length limitations.

\subsection{Short-path cell milestones}

\subsubsection{At optical wavelengths}

The pioneering work by Campbell \& Walker (1979) to measure RV was conducted with a hydrogen fluoride absorption gas cell of $90 \mathrm{~cm}$ length (Campbell et al. 1988). This comparably long cell vessel was accomodated in the converging beam of the $3.6 \mathrm{~m} \mathrm{CFHT}^{3}$ Coudé focus, feeding the $R=40000$ spectrograph. The vessel length (ie. the path length) was chosen so that the HF absorption lines are moderately strong ( 70\% depth for the stronger lines), following the principles outlined in Sec. 10.1. A low gas pressure was mandatory at the same time $(\sim 0.5 \mathrm{~atm})$ to prevent unduly pressure broadening of the line widths (Campbell \& Walker 1979), and as a safety measure to prevent gas outburst in the case of leaks (HF is highly toxic).

Only the CFHT's Coudé room provided the necessary benefits to host the HF cell apparatus, as it a) offered extended physical space to host the cell tube in the optical beam (plus auxiliary gas handling equipment), b) combined with a slow $\mathrm{f} / 20$ beam to allow undisturbed passage through the cell; and c) a thermally and mechanically isolated environment to minimize disturbances.

From the point of view of the optical setup, the gas cell acts in a purely passive way, and introduces no elements with optical power into the optical train (neglecting the potentially, small dispersing effects of the saphire windows). The HF cell approach by Campbell \& Walker emphasizes that an HF reference gas cell requires dedicated, extensive space. Such

\footnotetext{
${ }^{3}$ Canada-France-Hawaii Telescope
} 
an approach is only feasible between telescope and instruments with appropriate properties concerning dimensions and focal ratio.

The limitations imposed by the available space (and subsequently, by the prevailing focal ratio) pose a serious difficulty when a calibration gas cell is desired, particularly at existing facilities whose optical layout is not to be changed completely. Marcy \& Butler (1992) were able to overcome these difficulties for the Hamilton spectrograph $(R=62000$; Vogt 1987) at Lick Observatory, attached to the (common) Coudé foci of the $3 \mathrm{~m}$ Shane telescope and the $0.6 \mathrm{~m} \mathrm{CAT} 4$. By developing the "iodine method" (Marcy \& Butler 1992; Butler et al. 1996), they identified molecular iodine gas to have suitable characteristics for wavelength reference calibration in an optical wavelength window $(500-600 \mathrm{~nm})$ at low pressure, and at short optical path length $(\sim 10 \mathrm{~cm})$. Iodine $\left(\mathrm{I}_{2}\right)$ is much easier to handle than HF (non-lethal, only mildly corrosive), and has evolved as a standard tool in gas cell calibrated RV work in the visible. RV precisions of $3 \mathrm{~m} / \mathrm{s}$ are routinely obtained over long timescales, at different high-resolution instruments, using $\mathrm{I}_{2}$ gas cells.

As with $\mathrm{HF}$, however, $\mathrm{I}_{2}$ also requires being heated to $50^{\circ} \mathrm{C}$ (compared to $100^{\circ} \mathrm{C}$ for $\mathrm{HF}$ not to polymerize) in order to maintain a vapor state. The iodine approach is superior over $\mathrm{HF}$ also as the former provides absorption lines suitable for wavelength calibration in a $\sim 1300 \AA$ wide wavelength range, whereas this range is only $\sim 100 \AA$ in the case of HF. The downside of this an increase in complexity: because the HF lines are not strongly blended with stellar lines for the solar-like target stars selected by Campbell, Walker \& Yang, they are thus straightforward to measure from the composite spectrum (HF transmission + stellar spectrum, see Fig. 10.1). In contrast, the line abundance and density of iodine is much higher than in the case of $\mathrm{HF}$, and blends are omnipresent (blended $\mathrm{I}_{2}$ as well as $\mathrm{I}_{2}$-stellar blends). As a result, the analysis of $\mathrm{I}_{2}$ lines in the composite spectra involves sophisticated modelling of both the gas absorption lines and the stellar lines, together with solving for the instantanous point spread function of the instrument (Valenti et al. 1995; Butler et al. 1996). The PSF modelling, however, yields an important boost in precision, as it allows to account for eg. temporal variations of the slit illumination.

\subsubsection{At near-infrared wavelengths}

With the advent of planet search programmes that aim to utilize radial velocity measurements at longer wavelengths, considerable interest has focussed on efforts to extend the usability of the gas cell calibration method into the NIR wavelength regime.

Among others, Mahadevan \& Ge (2009) explored the suitability of commercially available molecular absorption gas cells for use in astronomical spectrographs, targeting at the detection of terrestrial mass planets around M-dwarfs. They present a concept to employ four standardized NIST ${ }^{5}$ gas cells, primarily used as standard wavelength reference materials in the telecommunication industry, to obtain wavelength calibration in the NIR $H$-band $(\sim 1.45-1.8 \mu \mathrm{m})$. These cells are made of simple tubes and are of various lengths $(5-20 \mathrm{~cm})$, and kept at different low and high pressures $\left(33-1330\right.$ mbar); filled with ${ }^{12} \mathrm{C}_{2} \mathrm{H}_{2}$ (Swann \& Gilbert 2000), $\mathrm{H}^{13} \mathrm{C}^{14} \mathrm{~N}$ (Swann \& Gilbert 2005), ${ }^{12} \mathrm{C}^{16} \mathrm{O}$, and ${ }^{13} \mathrm{C}^{16} \mathrm{O}$ (Swann \& Gilbert 2002), respectively (cf. Table 1 in Mahadevan \& Ge 2009). To achieve a wavelength coverage of $120 \mathrm{~nm}(1.51-1.63 \mu \mathrm{m})$ in the $H$-band, all four of these standard reference cells need to be employed in series, providing a total of $\sim 190$ absorption lines for calibration.

\footnotetext{
${ }^{4}$ Coudé Auxiliary Telescope

${ }^{5}$ United States National Institute of Standards \& Technology
} 

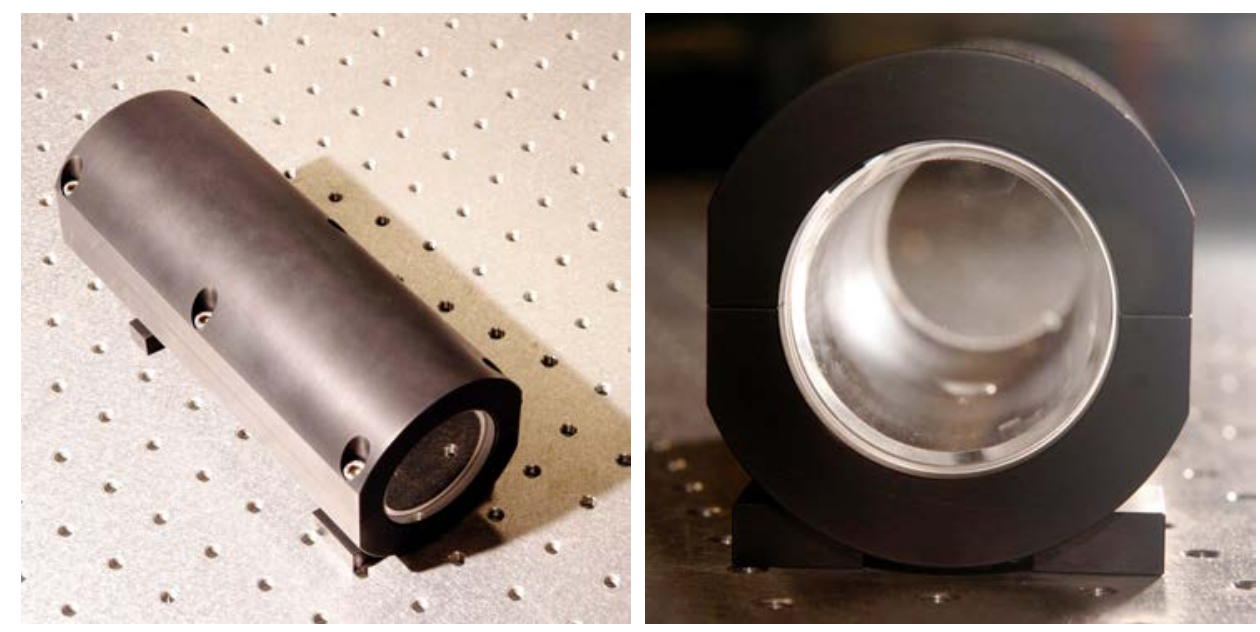

Figure 10.2.: Ammonia gascell for CRIREs deployed by Bean et al. (2010). The glass tube is $17 \mathrm{~cm}$ long, with a diameter of $50 \mathrm{~mm}$. The small inlet on the tube bottom is the filling inlet, prior to sealing. Image credit: the author.

Such a setup renders an application feasible only at a fiber-fed spectrograph, so that the telescope beam does not have to pass through the cells over a physical cell length of more than one meter, which would require a (near-)collimated beam over this distance ${ }^{6}$ A fiberfed application as proposed by Mahadevan \& Ge, however, does not allow the starlight to simultaneously pass the chain of cells (eg. for reasons of efficiency). Instead, a secondary calibration fiber is required, that feeds the reference imprints of the molecular gases into the dispersing spectrograph. As no stellar light is passing through the gases, the cells (and thus the calibration fiber), in turn, need to be properly illuminated by a suitable continuum source, which introduces further difficulties (eg. white-light coupling into the fiber; see the discussion in Mahadevan \& Ge 2009).

Hence, the Mahadevan \& Ge concept appears as a viable option for dedicated, stabilized fiber-fed spectrographs operating in the $H$-band. The approach is, however, more similar to the ThAr HCL technique than to the iodine method in the visible. Reference lines as wavelength markers are tracked simultaneously with stellar observations, but the spectral imprints do not share an identical optical path in the instrument, and are thus not co-located on the detector - so a stabilized instrument is called for to achieve a high precision wavelength calibration comparable to that obtained in the visible (cf. Sec. 9.1.2). Likewise, due to the different illumination conditions, the PSFs of the stellar object and the calibration fiber can be expected to differ (over time and by object), so that the instrumental line profiles of the stellar and of the gaseous contributions do not match. As a result, the attainable RV precision is narrowed, compared to a gas cell illuminated by stellar light.

More recently, Bean et al. (2010) have successfully managed to adapt the gascell calibration technique for use in the $K$-band range. These authors identified ammonia $\left({ }^{14} \mathrm{NH}_{3}\right)$ as a capable wavelength calibrator for a RV planet search programme around M-type stars, providing reference lines in a $36 \mathrm{~nm}$ wavelength band around $2.3 \mu \mathrm{m}$. Bean et al. (2010)

\footnotetext{
${ }^{6}$ Insertion of the proposed cells in series into the optical path in front of a spectrograph would require a very slow focal ratio, in order not to vignette the field of view at a mid- to large aperture telescope, and in order not to render the cell diameters unfeasably large.
} 
constructed an ammonia gas cell specifically for CRIRES $(R=100000)$ at the VLT. Their design considerations are driven by the wavelength regime of interest, ie. by finding an optimal solution to match the competing agents discussed in Sec. 10.1 (notably stellar RV content, availability of molecular calibration line coverage, and paucity of telluric perturbances). The cell realized is limited by the physical space allowances given by the mechanical and optical setup between the CRIRES spectrograph and the telescope feed, which constrains the conceivable line depth, so that the length of the calibration cell device is restricted to $17 \mathrm{~cm}$, with an according pathlength (Fig. 10.2). The cell vessel is thereby hosted in front of all the instrumental optics, including the adaptive optics (AO) module; hence allowing to track related instrumental drifts. This also means that the stellar PSF serves to illuminate the gas, so that the observed target and the reference spectral imprint share the identical optical path. Ammonia serves as a wavelength standard in laboratory physics (Urban et al. 1989; Yurchenko et al. 2005), and its transmission spectrum is relatively insensitive to temperature variations (Huang et al. 2008); enabling Bean et al. (2010) to achieve precision calibration without temperature stabilization of the gas.

While CRIRES is not an extremely stabilized instrument and neither fiber-fed (cf. Sec. 11.2.1), Bean et al. (2010) were able to demonstrate a RV precision of $\sim 5 \mathrm{~ms}^{-1}$ over one year, employing their cell. Ubiquitous telluric absorption lines throughout the $K$-band result in the presence of telluric features $\left(\right.$ eg. $\left.\mathrm{H}_{2} \mathrm{O}\right)$ also in the wavelength window covered by ammonia at $2.3 \mu \mathrm{m}$. These telluric lines need to be treated in the modelling process. As these features are variable in time, and dependent on the site and on the instantanous atmospheric conditions, a careful treatment by means of spectral synthesis (Seifahrt et al. 2010) is mandatory in order not to compromise the wavelength calibration, and hence the RV precision. The Bean et al. approach, based on a short ${ }^{14} \mathrm{NH}_{3}$ cell at low pressure ( $p=50$ mbar), thus involves instantanous modelling of the stellar spectrum, the $\mathrm{NH}_{3}$ transmission spectrum, and of the telluric absorption spectrum, for a given observation. As a consequence, and given the difficulties to determine precise input parameters at the time of observation for the telluric modelling, the RV precision attainable with the ammonia cell and CRIRES is clearly limited by the presence of telluric contaminants within the selected wavelength region (Bean et al. 2010). The CRIRES $\& \mathrm{NH}_{3}$ setup nevertheless demonstrates the potential of the gas cell technique in combination with a long-slit, high-resolution NIR spectrograph, yielding RV precisions approaching those obtained at optical wavelengths.

Based on the pioneering technique demonstrated on CRIREs, Anglada-Escudé et al. (2012) presented a method to optimize gas absorption cells in the NIR, and manufactured methane based cells using different isotopologues for use in the $K$-band. Their simulations show that methane $\left(\mathrm{CH}_{4}\right)$ is a beneficial reference gas with well measured lines (Nai-Cheng et al. 1981), superior over ${ }^{14} \mathrm{NH}_{3}$ in the $K$-band for RV works. By exploring the methane isotopologues ${ }^{13} \mathrm{CH}_{4}$ and ${ }^{12} \mathrm{CH}_{3} \mathrm{D}$, telluric blends can be effectively avoided. $\mathrm{As}{ }^{12} \mathrm{CH}_{4}$ is a prevalent species in Earths' atmosphere, the reference gas' line systems must avoid to blend with those from the (variable) telluric molecules. A wavelength shift of $8 \mathrm{~nm}$ in the case of ${ }^{13} \mathrm{CH}_{4}$ (compared to the natural ${ }^{12} \mathrm{CH}_{4}$ ) makes for a sufficient separation of the line systems, so that reference and telluric lines can be disentangled (Anglada-Escudé et al. 2012). Based on this convenient wavelength shift, Anglada-Escudé et al. designed reference gas cells for Cshell (Greene et al. 1993) on NASA's IRTF ${ }^{7}$. They report a RV precision on a M7 giant star of $35 \mathrm{~m} / \mathrm{s}$ over several nights, utilizing a temperature controlled ${ }^{13} \mathrm{CH}_{4}$ cell. A performance better than $5 \mathrm{~m} / \mathrm{s}$ can

${ }^{7}$ NASA Infrared Telescope Facility, Mauna Kea, Hawaii 
be expected at a higher resolving power and larger wavelength coverage as with CsHELL ( $R=46000, \Delta \lambda=6 \mathrm{~nm}$ ). The performance of the tested cell is again strongly hampered by the space limitations imposed by the clearance in front of the spectrograph entrance window, fixing the device's length to $12.5 \mathrm{~cm}$. This path length is too short to provide useful reference ${ }^{13} \mathrm{CH}_{4}$ features in eg. the $H$-band, where numerous lines exist but are too shallow given the pressure (275 mbar) optimized for deep lines in the $K$-band.

The same applies for the methane isotopologue ${ }^{12} \mathrm{CH}_{3} \mathrm{D}$, also tested by Anglada-Escudé et al. ${ }^{12} \mathrm{CH}_{3} \mathrm{D}$ provides a much increased line density over ${ }^{13} \mathrm{CH}_{4}$ (and ${ }^{12} \mathrm{CH}_{4}$ ), but at $10 \mathrm{~cm}$ path length the line depth is not sufficient in either band given CsHELL's resolving power, even at an enhanced pressure of $\sim 375$ mbar (Anglada-Escudé et al. 2012). Such pressure would be too high to deliver suitably sharp lines at a $R \sim 10^{5}$ instrument, but ${ }^{12} \mathrm{CH}_{3} \mathrm{D}$ remains as a promising option for versatile absorption gas cells with longer path lengths. ${ }^{12} \mathrm{CH}_{3} \mathrm{D}$ may also be combined with eg. ${ }^{14} \mathrm{NH}_{3}$, to further increase the line density and wavelength coverage in the $H$-band, and potentially in other wavelength regions. I will readdress the latter two points in Chapter 11.

Mixtures of gases for astronomical reference gas cells have also been explored by Valdivielso et al. (2010) and D'Amato et al. (2008). The former study investigates 18 molecular single gases for the presence of suitable absorption bands in the $0.8-2.5 \mu \mathrm{m}$ range. Their lowresolution NIR spectroscopy identifies 11 out of the 18 species with absorption features in the above wavelength range. Mixtures are reported that include ammonia, hydrocarbons, acetylene, nitrous oxide, and (chloro-)methane, with absorption bands mainly in two windows in the $H$-band (where telluric contamination is also reduced). None of the mixtures presented by Valdivielso et al. (2010) show usable features in the $Y$ and $J$-bands. This is because a) there is no known gas with strong and dense absorption in these ranges, and b) the existing small contributions (by chloromethanes and hydrocarbons) are too shallow and infrequent, given the considered cell length of $\sim 10 \mathrm{~cm}$ (Valdivielso et al. 2010). In turn, their gas mixtures are rich in absorption lines in the $K$-band, however, at the investigated pressure (atmospheric cell pressure) the lines are unresolved due to blending and pressure broadening.

On the other hand, D'Amato et al. (2008) have constructed a calibration cell containing $\mathrm{HCl}, \mathrm{HBr}$, and $\mathrm{HI}$ for the $0.95-2.4 \mu \mathrm{m}$ range. Their long device $(50 \mathrm{~cm})$ at a total pressure of approximately an atmosphere provides absorption bands in four windows of 80 to $160 \mathrm{~nm}$ width centered around 1.56, 1.77, 2.02, and $2.30 \mu \mathrm{m}$, owing to $\mathrm{HCl}, \mathrm{HI}, \mathrm{HBr}$, and $\mathrm{HI}$, respectively. Pressure broadening seems to be an issue for applications at high resolving powers $(R>50000)$ for such a cell, in line with low line densities and handling issues of the involved gases (eg. dissociation, corrosiveness; need for active cooling). The gas mixture of halogen hydrates does not have features in below $1.5 \mu \mathrm{m}$, ie. in the $Y$ and $J$-bands. The concept reported by D'Amato et al. (2008), with its increased path length of $50 \mathrm{~cm}$, requires special considerations for the instrument to be used with $\left(\mathrm{GIANO} / \mathrm{TNG}^{8}\right)$. The spectrograph design has to incorporate such space requirements in front of its entrance window, and provide a slow focal ratio beam to pass the cell accordingly (Bruno et al. 2006).

\subsection{Shortcomings}

The examples outlined above emphasize that current gas absorption cell technology cannot provide an ideal wavelength calibrator.

\footnotetext{
${ }^{8}$ Telescopio Nazionale Galileo, Roque de los Muchachos Observatory, La Palma, Spain
} 
First, no known gas or combination of gases exist that offer continuous line coverage at high line densities over a wide wavelength range, i.e. neither over the entire optical domain nor over the entire NIR domain, or major parts of it. Instead, gas absorption line bands populate rather narrow spectral windows, according to their ro-vibrational transition energies. A simple molecular gas thus cannot satisfy a line density and coverage comparable to that of HCLs (eg. ThAr).

Second, applications of absorption gas cells at astronomical spectrographs are limited to short pathlengths $(10-20 \mathrm{~cm})$, as physical space along the telescope beam in front of the instrument is very limited (for reasons of optical throughput, complexity, and size). A short pathlength implies a higher cell pressure in order to obtain sufficient line depths, but increased gas pressure in turn fosters unwanted line broadening. Concepts for longer cell pathlengths to increase gas selection flexibility and to allow for lower pressures require a dedicated design of and implementation at the instrument (eg. $50 \mathrm{~cm}$, D'Amato et al. 2008), at the cost of instrument versatility and expenses. Fiber-fed spectrographs are, in principle, not limited to short pathlengths if not the telescope beam is transmitted through the reference gas, but instead the fiber is coupled into the gas absorption cell. This is at the heavy cost of coupling efficiency and throughput, and impedes the option to take the calibration cell out of the optical path (in fact limiting spectroscopy to specialized, RV applications).

Thus, wavelength calibration by a gas absorption cell should not be considered as the only means of wavelength calibration for an instrument with broad wavelength coverage. However, in selected bands a gas cell (or combinations of cells and or gases) currently provides the most precise wavelength calibration intrinsically attainable (at reasonable cost), simply owing to the simultaneous and identical optical path of stellar light and reference imprint.

Consequently, it is desirable that the usable wavelength range for calibration by a reference gas be maximized. One strategy towards this goal is to allow for longer pathlengths than are currently achieved, enabling an additional degree of freedom when finding an optimal gas cell calibrator. A cell pathlength of up to several meters does not per se increase the wavelength range covered by a gas' absorption lines, yet vastly increases the gas selection flexibility and enables lower pressures. Weak absorption bands with shallow lines at short pathlengths then become suitable calibration lines when their line depth is amplified by longer pathlengths. An aim of this work is to search for solutions to overcome the short pathlength limitation of gas absorption cells.

\subsubsection{Why White- or Herriott cells do not work}

In laboratory physics, multipass absorption cells such as White-cells (White 1942) or Herriottcells (Herriott \& Schulte 1965) are often in use. These cells are comprised of an optical cavity enclosed by spherical, concave mirrors with the same radius of curvature. The lightsource is either fed from close aside (White) or through a hole in one of the mirrors (Herriott). The beam is reflected several times by the adjacent mirrors, making for multiple traversals, and thus allowing for a long optical path lenght despite a small cavity. The working principle relies on the fact that the facing mirror surface is always in the focus of the preceeding reflection.

These cells are suitable for laser spectroscopy and for fiber-fed applications with no spatial field and point-source like light-sources. For an extended field of view such as with CRIRES, the principle can be applied only when a field point (on-axis) is selected (eg. fed into a fiber), and that fiber is then coupled to such a cell. Accordingly, the principle is at conflict with the CRIRES need for a resolved field. Other than for a single field point, any off-axis position would a) suffer greatly from spherical aberrations and out-of focus conditions (not meeting 
the spherical mirror's focal point), and would b) be needed to pass eg. the feeding hole, whose size cannot be large enough to accomodate the whole FoV.

The specific CRIRES requirements (space, AO, fixed arrangement, ...; see Sec. 11.2.4) pose additional difficulties. For these reasons, White- or Herriott type cells are not deemed applicable. 



\section{Novel absorption cell design}

\section{Contents}

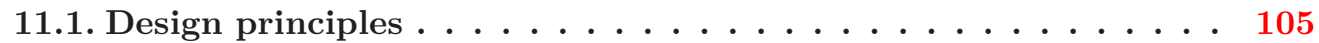

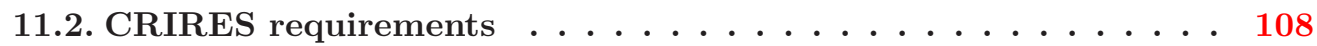

11.2.1. CRIRES key properties . . . . . . . . . . . . . . . . 108

11.2.2. Very Large Telescope key properties . . . . . . . . . . . . . . 111

11.2.3. Restrictions imposed by the VLT . . . . . . . . . . . . . . . 111

11.2.4. Restrictions imposed by the CRIRES instrument and its pre-optics . 114

11.2.5. VLT model . . . . . . . . . . . . . . . . . . . . . . . . 116

11.3. A long-path cell concept for CRIRES . . . . . . . . . . . 118

The collimation problem . . . . . . . . . . . . . . . . 119

11.3.1. First order concept . . . . . . . . . . . . . . . . . . . . 122

11.3.2. CRIRES model . . . . . . . . . . . . . . . . . . . . . . 123

Adaptive optics module . . . . . . . . . . . . . . 125

Derotator module . . . . . . . . . . . . . . . 125

Pre-slit optics module . . . . . . . . . . . . . . . . 126

11.3.3. NAGICS system overview _. . . . . . . . . . . . . . . 128

11.3.4. Optical design . . . . . . . . . . . . . . . . . . . . . . . 129

Fold-out sub-system . . . . . . . . . . . . . . . . . 131

Delay-line sub-system . . . . . . . . . . . . . . . . . . 132

Re-collimation and re-focuser sub-system . . . . . . . . . . 132

11.3.5. Gas vessel design . . . . . . . . . . . . . . . . . . . . . 134

In this chapter, a new design for gas absorption cells with long optical pathlengths is developed. Unlike conventional cell concepts in use at astronomical facilities, which resemble optically passive, tube-like designs, the new approach presented here facilitates active optical components to enable longer pathlengths than could be accomodated by a conventional cell. The aim is thus to allow for much longer paths traversing a calibrating reference gas, than the availabe physical space in the telescope beam permits.

The chapter is laid out as follows: I first discuss the general principles governing the construction of gas cells and their usage. Thereafter, the specific requirements and constraints of CRIRES at the Vlt are introduced. These set the stage for the discussion of the novel cell design approach and the problems that need to be overcome. Results of the newly developed gas cell concept are then presented and its components explained in detail.

\subsection{Design principles}

The primary driver that motivates the development of a new gas cell design is the prospect of very long absorption pathlengths. Following the principles discussed in Chapter 10, the 
distance available for the beam of light to traverse a reference gas shapes the line depths of the spectral imprint by the absorbing gas at a given density (Lambert-Beer law). The line strengths, in turn, define the fidelity of the lines and wavelength positions of the reference absorption lines can be determined with (among other parameters such as line sharpness, line density, line blending; cf. Sec. 10.1). Strong line depths (avoiding saturation, of course) are thus one of the crucial ingredients to achieve high-precision wavelength calibration. Consequently, the pathlength through an absorption gas is an important parameter in the optimization process of a gas cell calibration strategy. Leaving the pathlength as a free parameter is beneficial for two major reasons (see Sec. 10.3).

1. The choice of the reference gas is not limited to strong absorbers (implying short pathlengths), hence gases and wavelength ranges with weaker lines become usable.

2. Lower gas pressures can be realized, minimizing the perturbing effects of pressure broadening on the wavelength calibration quality (sharper reference lines).

The new gas cell concept developed in this work thus strives to maximize the achievable absorption pathlength.

The concept is developed with the following basic design principles:

- The calibration cell is to provide simultaneous wavelength calibration by means of a transmission spectrum, superimposed onto the stellar observations. The reference gas is thus to be placed into the telescope beam, so that the spectral reference imprint and the stellar light share the same optical path through the spectrograph. Both the stellar spectrum and the transmission spectrum are thus co-located on the detector.

- The pathlength of the gas cell shall be longer than that of a conventional gas cell. This means that the limitation in length of a conventional approach shall be overcome, enabling long pathlengths.

- At the same time, the mechanism is to fit into the availabe space along the optical beam, between the telescope and the spectrograph. Hence, the mechanical setup of the telescope and of the instrument is kept unchanged, so that no relocation of the instrument is necessary.

- The calibration cell shall be optional during operations of the spectrograph. The cell thus shall be designed so that observations with the cell taken out of the optical beam are feasible. Taking out the cell shall not require modifications to the instrument.

- Concurrently, the cell mechanism shall not alter the telescope beam properties entering the spectrograph. The optical input properties for the instrument shall thus remain unchanged.

The constraints imposed by these design principles render an application of such a cell at a fiber-fed instrument unfeasible, in which the cell would be placed behind the telescope focal plane, as in the latter the fiber head is mounted to couple light into a fiber. For such a setup, the gas cell is illuminated by the fiber, coupled into the cell on its way to the spectrograph. No simple removal of the cell device is then feasible, unless the cell is bridged by some sort of other fiber feed, or unless the complete fiber line from fiber head in the focal plane to 


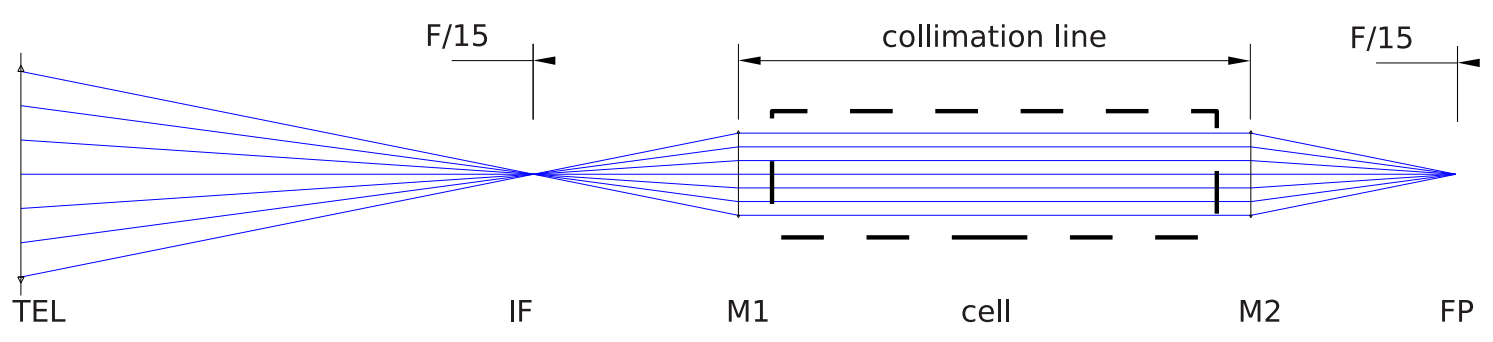

Figure 11.1.: Concept for a conventional long-path gas cell design by means of a collimation line. The telescope beam with a given focal ratio is collimated at a convenient location after the telescope intermediate focus (IF) by a mirror (M1). The collimated beam (at a convenient diameter) can extend over a long distance as needed to insert a tube-like gas cell, given enough space. A second mirror (M2) re-focuses the beam into the focal plane with the desired focal ratio (back to $\mathrm{f} / 15$ in this example). In practice, such an idealistic paraxial approach requires very slow focal ratios and minimal field of view to manage aberrations, eg. an optical fiber as a point source input in the IF.

the instrument is switched to an additional, continuous connection. ${ }^{1}$ In any case, a fiber-fed gas cell concept suffers from the limitations discussed in Sec. 10.2.2. We will later see that for the case of fiber based instruments, much simplified gas cells can be conceived. As a fiber connected cell can be easily displaced from the telescope beam (it can be located in any convenient place), physical space limitations do not strictly apply, and a conventional cell approach appears reasonable (with the respective fiber coupling).

The case of a fiber-fed gas cell is thus not further considered in the present development. However, it is beneficial to place a gas cell into the optical beam, before the stellar light is coupled into a fiber (cf. Sec. 10.2.2). The new gas cell concept already comprises such a case.

The principles above envision an application of the new gas cell design primarily at existing facilities. This is because for newly designed instruments, the principal goal - an increased optical pathlength of the gas cell - can be realized, in most cases, more conveniently by implementation of a large distance between telescope focus and spectrograph entry in the first place. This distance can be easily employed to accomodate a gas cell with the respective long pathlength, if the instrument design considerations allow.

As an example, given enough space availabe, collimation of the telescope beam with successive transmission through an arbitrarily long, conventional tube-like gas cell (followed by some re-imaging unit), is an obvious solution but requires that the telescope focus and the instrument can be designed and placed accordingly. Fig. 11.1 illustrates such a case. I will revisit this example in more detail in Sec. 11.3 to highlight selected design difficulties for the application scenario at existing, immutable instrumentation.

The major challenge in the design of a long-path cell for present-day spectrographs in operations is thus to beat the restrictions imposed by the (non-)available space in the optical layout. A solution to the problem must, at the same time, consider reasonable complexity and managable cost.

\footnotetext{
${ }^{1}$ In reality, most fiber-fed spectrographs employ additional devices between the telescope focal plane and light injection onto the spectrograph slit, eg. a fiber scrambler and -shaker. Any such device further necessitates a changing connection once the gas cell apparatus is taken out.
} 


\subsection{CRIRES requirements}

RV observing programmes aimed at detecting and characterizing low-mass exoplanets require a long-term RV precision on the order of $1 \mathrm{~m} / \mathrm{s}$. An absorption gas cell, given that it can track instrumental drifts and instabilities on the same level of precision, thus needs to deliver an intrinsic overall wavelength precision on the same level. This is the sole purpose of the gas cell technique, and has proven feasibility (see Chapter 10).

CRIRES $^{2}$ at the Vlt (Käufl et al. 2004, 2006a,b, 2008) is the only instrument availabe in the NIR with a resolving power as high as $R=10^{5}$, which offers superb benefits to conduct RV monitoring of low-mass planets around very late-type stars: it provides the highest resolving power availabe in the NIR, has a wavelength coverage of $0.9-5 \mu \mathrm{m}$ (with a simultaneous coverage of $\sim \lambda / 70$ ), is mounted on an $8 \mathrm{~m}$ class telescope, and features an adaptive optics module (Paufique et al. 2006). It is thus an ideal instrument for precision velocimetry to greatly benefit from the gas cell calibration technique. In fact, a dedicated (short-path) gas cell has been successfully deployed (Bean et al. 2010) as discussed in Sec. 10.2 for a planet RV search programme. The general CRIRES wavelength calibration plan is based on a ThAr HCL (below $2.5 \mu \mathrm{m}$ ) and night sky emission and telluric absorption lines (above $2.5 \mu \mathrm{m}$; see also Sec. 9.1.2). Standard calibration also comprises two short-path $(\sim 15 \mathrm{~cm})$ gas cells filled with $\mathrm{N}_{2} \mathrm{O}$ and $\mathrm{CO}$, respectively. They can be used for wavelength calibration in simultaneous (illuminated by the stellar light) or sequential mode (illuminated by a halogen lamp before/after the actual observations). $\mathrm{N}_{2} \mathrm{O}$ is a wavelength standard for redwards of $3.5 \mu \mathrm{m}$, and also provides some weak absorption bands in the range 1.65-2.10 $\mu \mathrm{m}$ (NIST; Rothman et al. 2009). CO has six windows with absorption bands between 2.3-5.1 $\mu \mathrm{m}$. Both $\mathrm{N}_{2} \mathrm{O}$ and $\mathrm{CO}$ are also telluric species, so that for simultaneous precision calibration, these gases are inadequate. Additionally, for RV studies the wavelength ranges covered by $\mathrm{N}_{2} \mathrm{O}$ and CO do not coincide with a high RV information content ("Q-factor", Bouchy et al. 2001) in low-mass stars. The existing standard CRIRES gas cells are thus not satisfactory, and motivate the present study to enable a long-path gas cell. Consequently, the new gas cell concept developed in this chapter specifically targets the CRIRES facility.

\subsubsection{CRIRES key properties}

The basic properties of CRIRES are summarized in Käufl et al. (2004) and on the instrument web-site. ${ }^{3}$ CrIREs is mounted at the Nasmyth focus of UT1, and installed on the Nasmyth platform co-rotating with the telescope (Fig. 11.2). The instrument consists of a "warm" part (pre-optics) and a "cold" part.

The warm pre-optics system is located in front of the spectrograph entrance window as seen from the telescope, and comprises an instrument derotator (the Nasmyth focus suffers from field rotation in an Alt-Az telescope mounting), the AO system, and a calibration unit which allows to place a short gas cell into the converging telescope beam, or inject light from calibration sources into the beam (via a folding mirror). This part is not thermally controlled, so it is subject to the environmental fluctuations as present in the telescope dome.

The cold part is housed in a cryogenic, evacuated vessel at $70^{\circ} \mathrm{K}$ (within $0.1^{\circ} \mathrm{K}$ ) and resembles the actual spectrograph. Light enters the vessel via a dichroic window (the optical red portion is directed to the $\mathrm{AO}$ system), passes a cold stop in a pupil plane (to suppress

\footnotetext{
${ }^{2}$ Cryogenic High-Resolution Infrared Echelle Spectrograph, mounted on UT1 (Antu) at the Very Large

Telescope of the European Southern Observatory, Paranal, Chile.

${ }^{3}$ http://www.eso.org/sci/facilities/paranal/instruments/crires/
} 


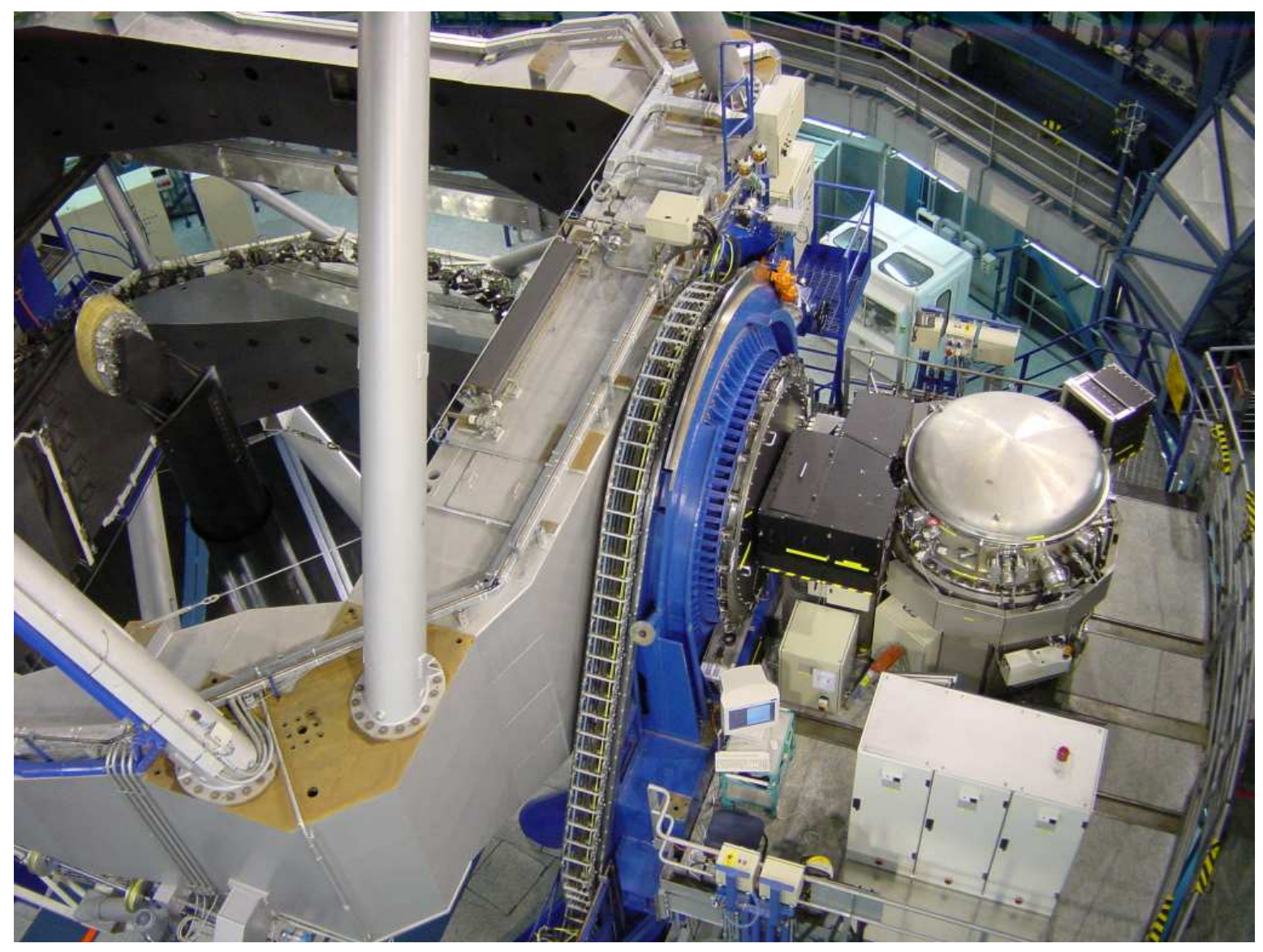

Figure 11.2.: The Cryogenic High-Resolution Infrared Echelle Spectrograph (CRIRES) at the VLT. The $\varnothing 3 \mathrm{~m}$ instrument cryostat vessel (silver) is mounted on the Nasmyth A platform of UT1 Antu. The covered box (black) between vessel and telescope mount (blue ring) hosts the (warm) pre-optics system, including calibration unit, instrument derotator, and AO module. The total instrumental platform load is $6200 \mathrm{~kg}$. The $8 \mathrm{~m}$ primary mirror of UT1 can be seen in the top left. Image credit: ESO

thermal background), and is focussed onto the entrance slit (0!' 2 or $0 . " 4$ projected slit width, yielding $R=100000$ or $R=50000$, respectively; projected slit length $\sim 40^{\prime \prime}$ ). Reflected light from the slit jaws is re-imaged towards a slit viewer detector array. For light passing the slit a ZnSe prism serves as a pre-disperser, followed by an intermediate slit to isolate a single echelle order. The order is then collimated and dispersed by an R2 echelle grating $(40 \times 20 \mathrm{~cm}$ in size; 31.6 lines $/ \mathrm{mm}, 63.5$ blaze angle; Käufl et al. 2004) as the high-resolution dispersion element. The collimator is formed by a three mirror anastigmat (TMA) in Littrow-mode, so the TMA also serves as the camera to focus the dispersed light onto the detector array. The $4096 \times 1024$ pixels detector mosaic (spectral $\times$ spacial, 512 active pixels in spatial direction) consists of four Raytheon Aladdin III InSb arrays ( $27 \mu \mathrm{m}$ pixel size; with three gaps of $\sim 283$ pixels) at a second $27^{\circ} \mathrm{K}$ cryogenic stage (stable to $\pm 0.1^{\circ} \mathrm{K}$ ), defining a projected pixel size of 0.'086 in the spatial direction (Käufl et al. 2004). Fig. 11.3 overviews the CRIRES optical design.

An instrument upgrade ("CRIRES+", Oliva et al. 2012; Follert et al. 2013) is envisioned and 


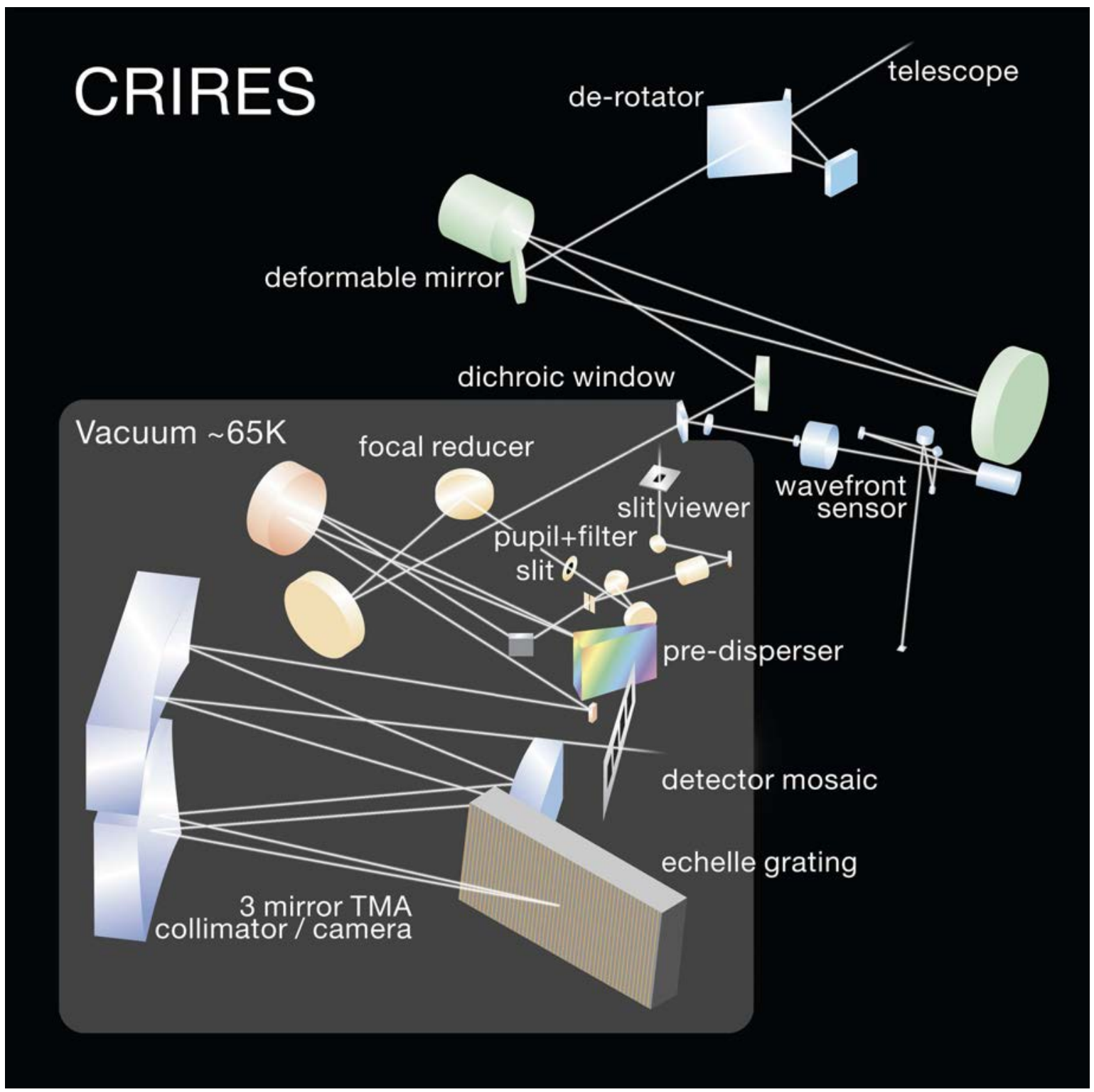

Figure 11.3.: Optical design of CRIRES. Light from the telescope passes an optical bench with the warm instrument part (immediately after the Nasmyth flange) with its calibration slide (not shown; holding, eg. a gas cell), the instrument derotator, and the adaptive optics module. A cryogenic vacuum vessel hosts the cold instrument part, consisting of pre-optics to focus the light onto the slit, and to image the slit, followed by a prism spectrometer serving as a pre-disperser, and the high-resolution echelle grating in Littrow mode. The dispersed spectrum is then imaged onto a detector mosaic. Image credit: ESO/Käufl et al. 2004

scheduled to expand the merits of the existing CRIRES design. The upgrade primarily aims at adding a cross-disperser in order to overcome the current small simultaneous wavelength coverage $(\sim \lambda / 70)$. Together with a new, larger detector mosaic the spectral coverage is increased by a factor of $\sim 15$, at the cost of a shorter slit length $\left(10^{\prime \prime}\right)$. 


\subsubsection{Very Large Telescope key properties}

The European Southern Observatory's Very Large Telescope (VLT) facility is comprised of four identical unit telescopes (UT1-UT4), located on Cerro Paranal in the Atacama desert in Northern Chile. Each UT serves a Cassegrain focus ( f f/13) and two stationary Nasmyth foci (f/15), plus a Coudé focus (f/47) with slightly different optical properties. In the context of an optically active gas cell approach, it is beneficial to simulate the VLT in a complete optical model. The optical telescope characteristics are documented in Dierickx et al. (1995) and in Rupprecht (2005). For our purposes (CRIRES on Nasmyth A), I only consider the properties of the Nasmyth focus.

The VLT features a primary mirror with a reflective diameter of $8.1859 \mathrm{~m}$. This M1 is of nearly parabolic shape, and has a radius of curvature (ROC) of $28.800 \mathrm{~m}$. The secondary mirror (M2) is a conic mirror of $1.116 \mathrm{~m}$ reflective diameter, sitting $\sim 12.4 \mathrm{~m}$ above the M1. A flat tertiary (M3) is tilted at $45^{\circ}$ to provide one Nasmyth focus at a time $6.800 \mathrm{~m}$ aside of the telescope axis. The total field of view is thus $30^{\prime}$, with an unvignetted field of $7.15^{\prime}$. The vignetting is due to the primary mirror, with $0.31 \%$ and $2.14 \%$ vignetting at $10^{\prime}$ and $20^{\prime}$, respectively. This results in a focal plane diameter of $1.0438 \mathrm{~m}$ (0.2496 m unvignetted), with the focal plane being curved (concave towards the telescope). The Nasmyth foci provide a focal ratio of $\mathrm{f} / 15,0.250 \mathrm{~m}$ after the beam leaves the Nasmyth adapter ring. Table 11.1 lists the optical parameters of the UT for the Nasmyth focus. The UTs are optimized for the optical to infrared spectral range, and are usually operated in a field stabilization mode in which the secondary's movement is controlled to compensate for wind shake etc, rather than auto-guiding via corrections to the telescope axis movements. The shape of the primary mirror is controlled by active optics (also used to change the M1 conic shape when switching between foci). Its (movable) probe is mounted on a pick-up arm reaching into the telescope's field of view at the position of the Nasmyth adaptor, redirecting light to the wave front sensor (for the active optics system) and the aquisition guide sensor (for guiding and instrument field selection). Fig. 11.4 shows a schematic of the telescope.

\subsubsection{Restrictions imposed by the VLT}

Any device to be installed between the VLT and the CRIRES instrument interfaces with the telescope on the one side, and the instrument or its pre-optics (eg. the AO system) on the other side. The major constraints imposed by the telescope is the focal ratio (f/15), and simply space limits. As for the latter, the back-focal distance (clearance between the unobstructed end of the Nasmyth adapter structure and the focus position) is only $250 \mathrm{~mm}$. This space is the principal location for any (short) calibration gas cell, and in fact the two CRIRES standard cells occupy this part. The availabe space is further diminished (see next paragraph) by a calibration mirror immediately after the Nasmyth adapter, leaving an effective $180 \mathrm{~mm}$ clearance between the calibration mirror unit and the focus. A mechanical sliding train fills this room to position eg. the standard cells (mounted on the slide) into the optical beam. The telescope beam comes to focus on the very edge of this calibration train. The telescope focusing mechanism can accomodate the focal plane position within $215-255 \mathrm{~mm}$ behind the adapter's rotator instrument mounting flange. Any mechanism to be installed here has to accept the f/15 ratio and to not physically extend beyond the nominal focus position (see below); the proximity to the focus also dictates the required dimensions for an unvignetted field $(55 \mathrm{~mm}$ clear diameter at a back-focal distance of $144 \mathrm{~mm})$. Fig. 11.5 illustrates the arrangement around the telescope focus. 


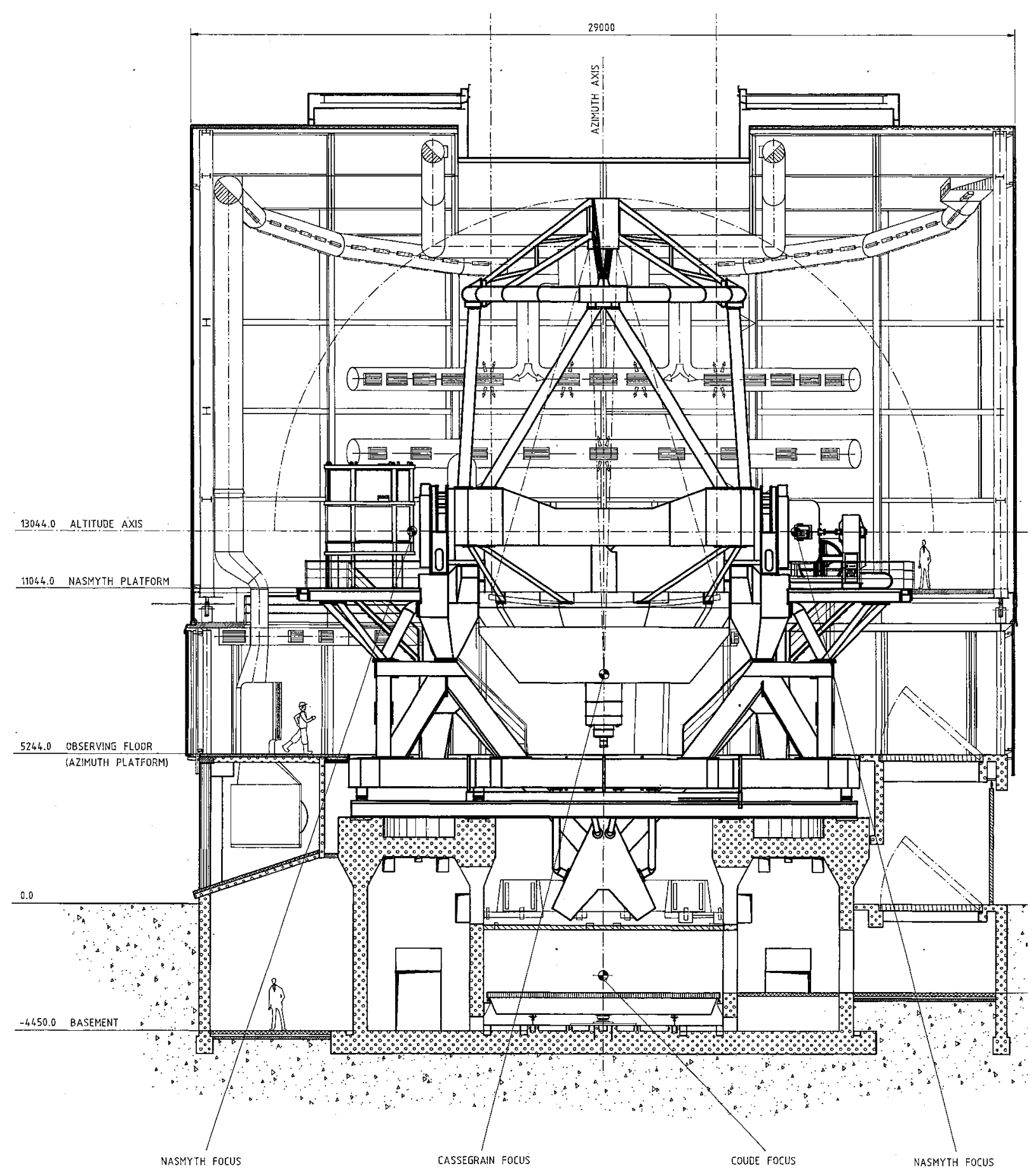

Figure 11.4.: Schematic drawing of the VLT unit telescope in its dome enclosure. The telescope structure (center) is indicated together with the path of incident light (dash-dotted lines) towards the Cassegrain and Nasmyth foci (labelled). The Coudé foci are redirected from inside each of the Nasmyth adapter rings (by means of a pick-up arm) via a five mirror train into the telescope basement (used for interferometry). CRIRES (not shown here) is mounted on the left Nasmyth platform. (adapted from ESO/ Rupprecht 2005) 


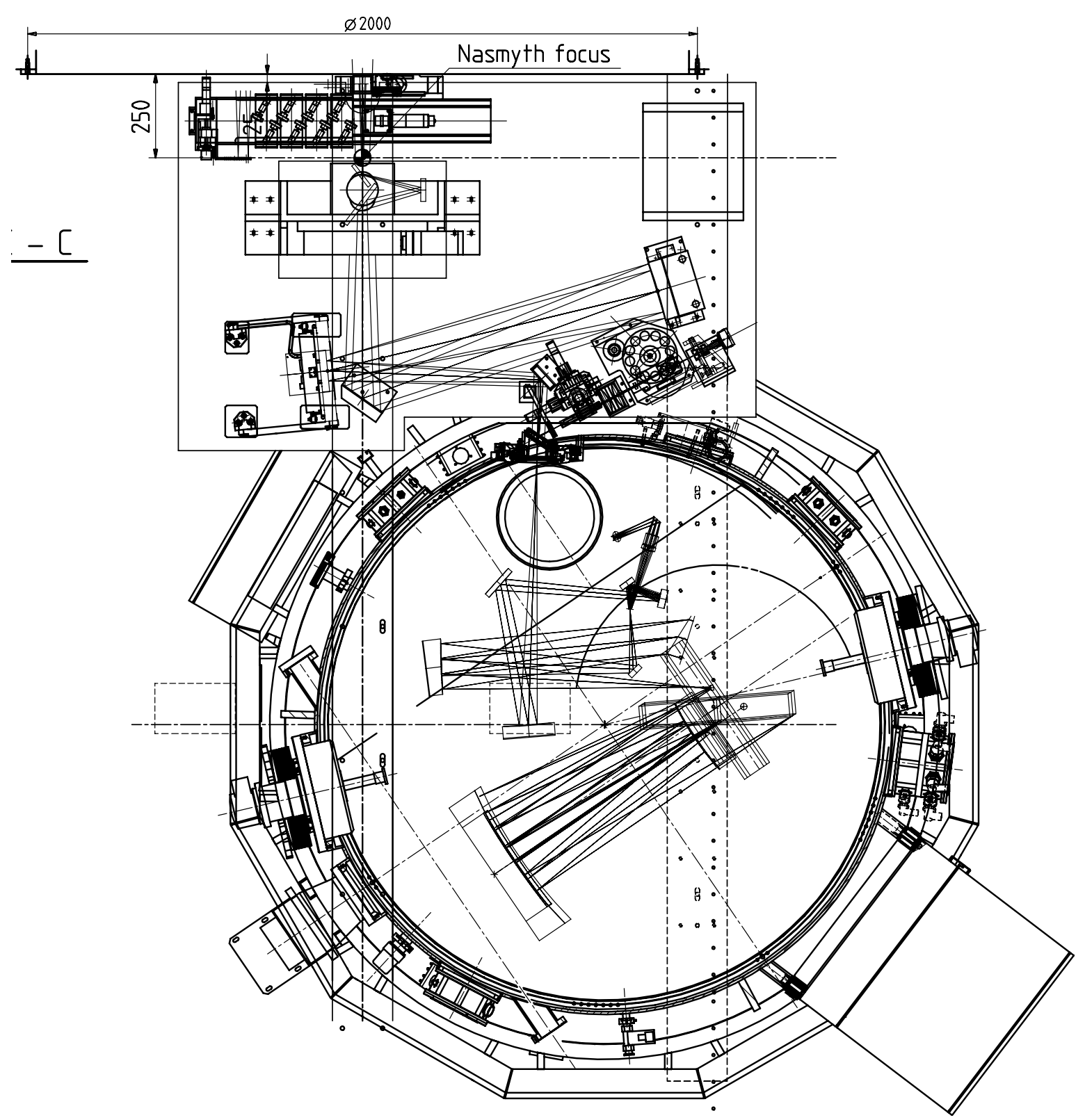

Figure 11.5.: Arrangement of the CRIRES spectrograph on the Nasmyth platform (section of drawing), seen in top view. The telescope adapter is at the top, below is the optical bench with the CRIRES warm optics (in oder of the optical chain: calibration mirror unit, calibration slide, de-rotator unit, adaptive optics module and wavefront sensor unit). The cold part is located in a cryogenic vacuum tank (bottom), with the main optical components and the light path shown (dimensions omitted). (adapted from ESO/VLT-DWG-14500-0-300003). 


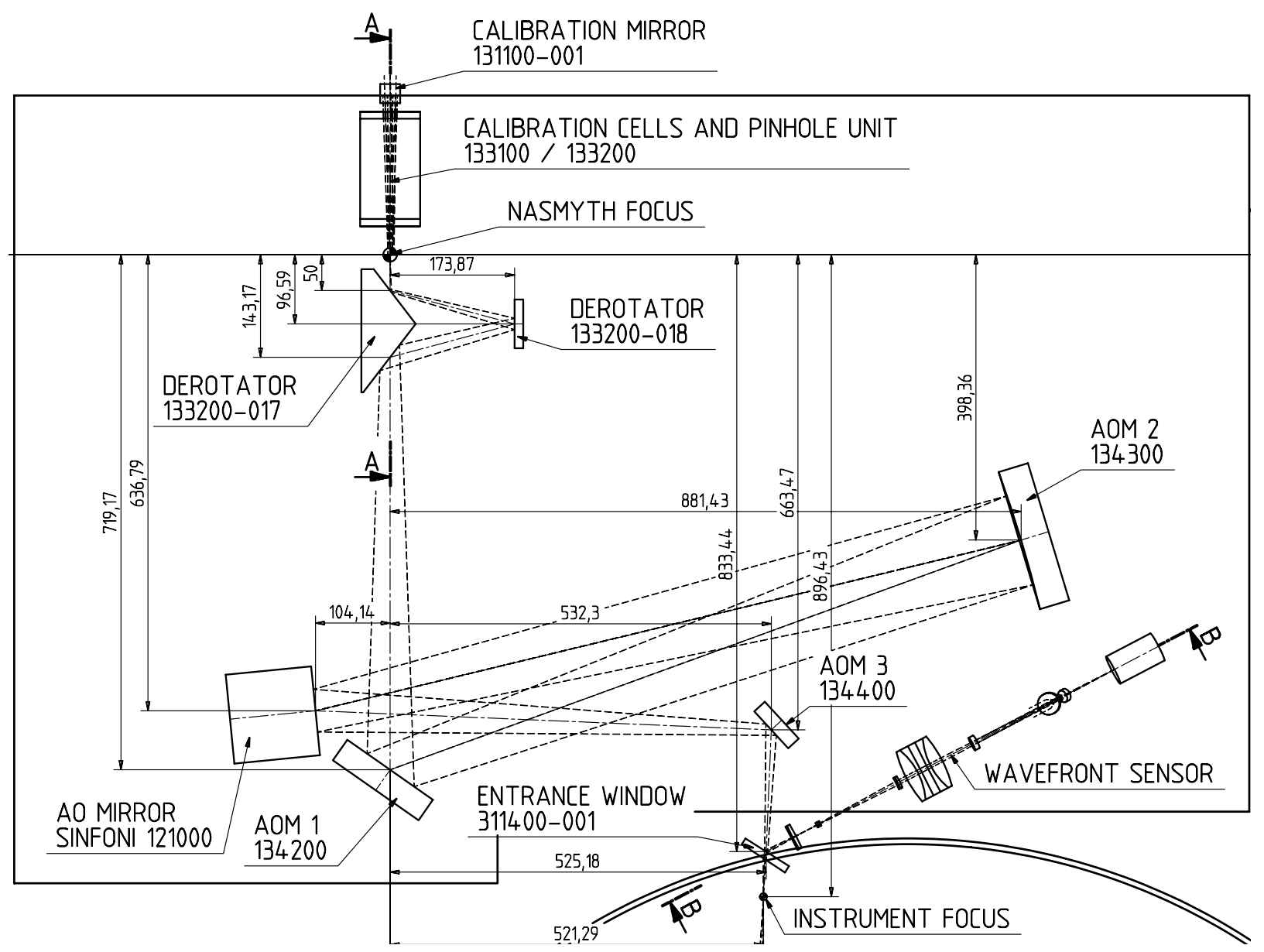

Figure 11.6.: CRIRES optical layout (detail). The telescope adapter flange is located to the top (not shown), $250 \mathrm{~mm}$ before the Nasmyth focus. Clearance and a calibration mirror unit occupy $70 \mathrm{~mm}$ after the flange, leaving only $180 \mathrm{~mm}$ space until the focus for a sliding train ("calibration cells") to host eg. gas cells. The instrument derotator unit is located immediately after the focus, followed by the AO module. The entrance window is a dichroic (reflecting the optical light to the $\mathrm{AO}$ wavefront sensor) to the vacuum tank and separates the cold instrument part in a cryogenic environment. After the instrument focus follows the CRIRES pre-slit optics and the spectrometer; cf. Fig. 11.5. (adapted from ESO/VLTDWG-14500-0-300002).

\subsubsection{Restrictions imposed by the CRIRES instrument and its pre-optics}

The Crires optical layout is on display in Fig. 11.6 (in parts). As reasoned in Sec. 11.1, a calibration gas cell device must be placed into the optical beam as early as possible in the optical train, in order to witness and thus make traceable instrumental effects from this location on until imprinted onto the detector in a spectrum's signature. The VLT-focus and the location of the CRIRES vessel must be assumed fixed and non-movable. Any re-location of the vessel or optical components therein must be considered too costly, and would imply a major redesign of the instrument and a change of the spectrograph properties. In this way, a simple re-design of the arrangement on the Nasmyth platform with the aim to increase the 
distance between telescope focus and instrument entrance window (ie. instrument focus) is out of scope. Such a solution would offer space to accomodate a collimated beamline, suitable for a long-path tube-like gas cell (cf. Sec. 10.3).

Thus, the instrument input parameters are required to remain unchanged. These constraints translate into the following requirements:

1. The CRIRES fore-optics inside the cold instrument part serves to a) provide an image of the telescope pupil. This pupil image is used to place a cold stop and filters (cf. Sec. 11.2.1), and must be maintained. Also, b) the fore-optics adapt the focal ratio at the slit to match the spectrograph camera. This means that an input f/15 (through the entrance window) is strictly required (Delabre et al. 2000). The full CRIREs field of view is $50 \times 50^{\prime \prime}$ (projected onto the slit). A reduced field is feasible, but also results in a reduced slit-length. The goal is thus defined to maintain the full FoV, whereas the requirement is a FoV no smaller than $5 \times 5^{\prime \prime}$ (H. U. Käufl, priv. comm).

2. The MACAO AO system (Paufique et al. 2006) is located on the warm bench just in front of the cryostat entrance window. The four mirror module operates at unit magnification (Delabre et al. 2000), and provides the f/15 input to the cold part optics. The input beam is thus also of $\mathrm{f} / 15$ (from the telescope), and any mechanism to be inserted before or after the $\mathrm{AO}$ needs to preserve this focal ratio. The $\mathrm{AO}$ system cannot be re-located without re-designing it as the instrument focus and the telescope focus are at defined positions. An important requirement concerns the AO deformable membrane mirror ("AO mirror" in Fig. 11.6). In order for the AO to function, an image of the telescope pupil needs to be placed onto the AO mirror surface. This is achieved by AOM1 and AOM2 (and the according distances). As the telescope pupil sits at the secondary (tip-tilt) mirror, the M2 must be imaged onto the AO mirror to ensure $\mathrm{AO}$ correction. If this condition is not preserved with a gas cell mechanism, the AO functionality for CRIRES goes astray.

The AO wavefront sensor also requires that optical wavelengths $(450-950 \mathrm{~nm})$ be transmitted up to the cryostat entrance window.

3. The instrument de-rotator is located just after the telescope focus (before the AO). Although it only consists of three flat mirror surfaces, and thus is not optically active, its dimensions (clear field of view) prohibit moving it towards the AO system (this would enlarge the accessible space around the telescope focus, to accomodate larger/longer calibration units).

4. Likewise, the calibration mirror system and the sliding train with calibration units are tied to their locations immediately after the telescope adaptor flange and before the derotator (ie. effectively between adapter and Nasmyth focus).

Hence, there exist only two potential locations to insert a calibration device into the optical train. This is, first, the existing calibration train slide immediately before the telescope Nasmyth focus. The space provided by the slide is $180 \mathrm{~mm}$ along the optical path (the slide carriage is $862 \mathrm{~mm}$ long, perpendicular to the optical axis; other gas cells and calibration units such as a pinhole and a polarimeter need to be accomodated on this carriage).

Second, there is some $577 \mathrm{~mm}$ nominal space between the last derotator optical surface and the first AO mirror. Given the mirror housings and the extended derotator mount, some $300 \mathrm{~mm}$ clearance remain. However, the AO deformable mirror is closely adjacent to the 
Table 11.1.: VLT optical design data for the Nasmyth configuration (f/15, effective focal length $12.000 \mathrm{~m}$ ). For details, refer to ESO technical report VLT-TRE-ESO10000-0526 (Dierickx et al. 1995).

\begin{tabular}{|c|c|c|c|c|c|c|}
\hline Surface & $\begin{array}{l}\mathrm{RoC}^{a} \\
{[\mathrm{~mm}]}\end{array}$ & $\begin{array}{c}\text { Distance }^{b} \\
{[\mathrm{~mm}]}\end{array}$ & $\begin{array}{c}\text { Diameter } \\
{[\mathrm{mm}]}\end{array}$ & $\begin{array}{l}\text { Tilt } \\
\text { [deg] }\end{array}$ & $\begin{array}{c}\text { Conic } \\
\text { constant }\end{array}$ & Comment \\
\hline OBJ & Infinity & Infinity & - & & 0 & WFM \\
\hline 1 & Infinity & 89134 & 8000 & & 0 & Entrance Pupil ${ }^{c}$ \\
\hline 2 & -28800 & -12396.43 & 8185.9 & & -1.00469 & TEL M1 ${ }^{d}$ \\
\hline STO & -4553.571 & 9896.429 & 1116.043 & & -1.66926 & TEL M2 \\
\hline 4 & Infinity & -6550 & 752.1417 & 45 & 0 & TEL M3 $3^{e}$ \\
\hline 5 & Infinity & -250 & 60.05316 & & 0 & Adaptor/Rotator flange edge \\
\hline IMA & 2089.6 & & 41.14407 & & 0 & Nasmyth focus $f$ \\
\hline
\end{tabular}

Notes.

(a) Radius of Curvature.

(b) Axial distance to next surface.

(c) The entrance pupil is located behind (ie. just below) the primary mirror.

(d) The Nasmyth configuration is maintained by the shape of M1, and controlled by the active optics. In the Cassegrain configuration, RoC and conic change for M1.

(e) M3 is flat, tilted, and elliptical with $866 \times 1242 \mathrm{~mm}$ (M3 is rotated off its tower parallel to the altitude axis during Cassegrain configuration, to liberate the Cassegrain hole in M1 through the M3 tower. This is depicted in Fig. 11.2).

(f) The image plane/focal plane diameter is given for a $50^{\prime \prime} \times 50^{\prime \prime}$ field of view.

first $\mathrm{AO}$ mirror, so that vignetting of it would occur (see Fig. 11.5). Accounting for the structure mount of the deformable mirror, approximately $200 \mathrm{~mm}$ usable space remain (exact dimensions are unknown due to a lack of available documentation).

The second option thus does not offer considerably more physical space, but additionally suffers from a larger diverging beam diameter, and obstructions from other close-by elements. Effectively, the calibration carriage remains as the only acessible location for an absorption gas cell mechanism.

\subsubsection{VLT model}

A complete optical model of the VLT is designed to foster accurate simulations of the optical properties and to enable a performance analysis of the entire system (telescope, gas cell mechanism to be designed, and warm pre-optics). The model incorporates the dimensions and parameters for the Nasmyth configuration as outlined in Sec. 11.2.2. The values are taken from Dierickx et al. (1995), reflecting the changes made to issue 1.B of that document. A prescription of the VLT-design is given in Table 11.1. Fig. 11.7 shows the optical layout and illustrates the coordinate system. Fig. 11.8 represents a 3D rendering to highlight orientation and arrangement.

This model correctly reflects the location, dimensions, and optical properties including aberrations of light and apertures until the Nasmyth focal plane for any desired wavelengths. Vignetting by the primary is taken into account (albeit this is only needed for fields of view exceeding $\sim 7^{\prime}$, much more than required by CRIRES). 


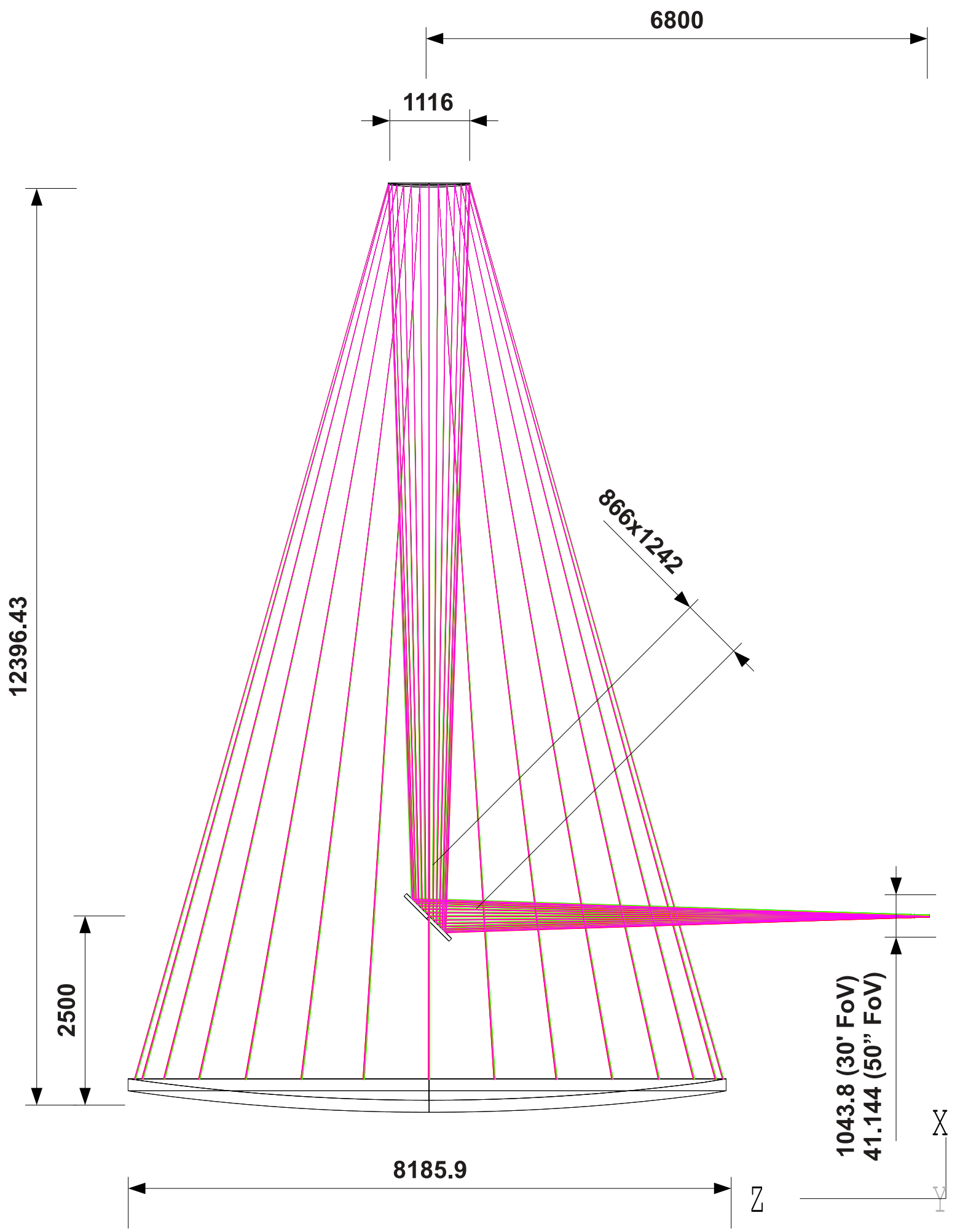

Figure 11.7.: Optical model of the VLT in Nasmyth setup. While M1 and M2 are circular surfaces, the flat M3 mirror is of elliptical shape. The focal ratio is $\mathrm{f} / 15$, the size of the focal plane is indicated for the full VuT FoV $\left(30^{\prime}\right)$, and for the $50^{\prime \prime}$ Crires FoV. Dimensions given in mm. 


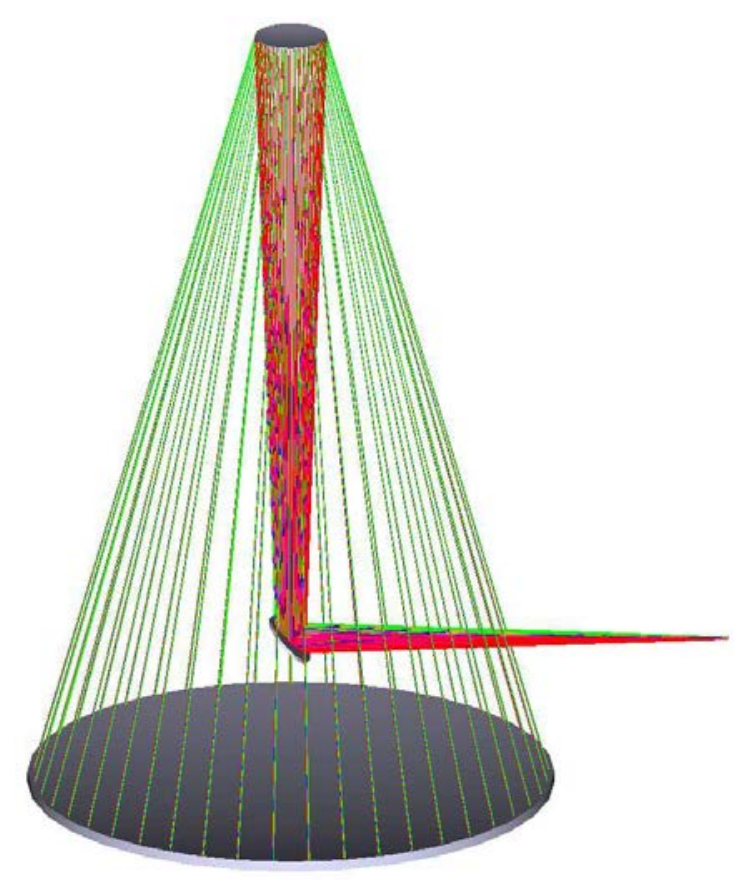

Figure 11.8.: 3D rendering of the optical VLT model. The colour of the light rays represents fans of rays from different field angles (cf. Fig. 11.9).

\subsection{A long-path cell concept for CRIRES}

The objective of this section is to design an optical mechanism to enable a long-path gas absorption cell at the CRIRES spectrograph. As identified in the previous sections, such a mechanism is only reasonably accomodated in the optical path before the telescope focus, at the location of the sliding calibration carriage.

The restrictions imposed by this location and the requirements for such a device, also discussed in the previous sections, must be met for the situation when the gascell device is in place (ie. in the optical path), and likewise when not in place (temporarily removed from the optical path).

The availability of only $180 \mathrm{~mm}$ along the path of light, together with the requirement for a long-cell, result in a fundamental design decision. The resulting approach is four-fold:

1. To fold the optical path out of the Nasmyth-instrument plane to gain space and access,

2. To condition the optical properties to employ a long-path cell, and

3. To fold the optical path back onto the original plane, with the same, restored properties as before the fold-out.

4. To avoid refracting elements as to not introduce chromatism and to satisfy the large wavelength coverage requirement $(\sim 0.65-5 \mu \mathrm{m}$; the $\mathrm{AO}$ requires at least some portion of the $R$ band to operate). Lenses are not feasible to be efficiently employed simultaneously over this entire wavelength range, so a purely reflective approach is chosen. 

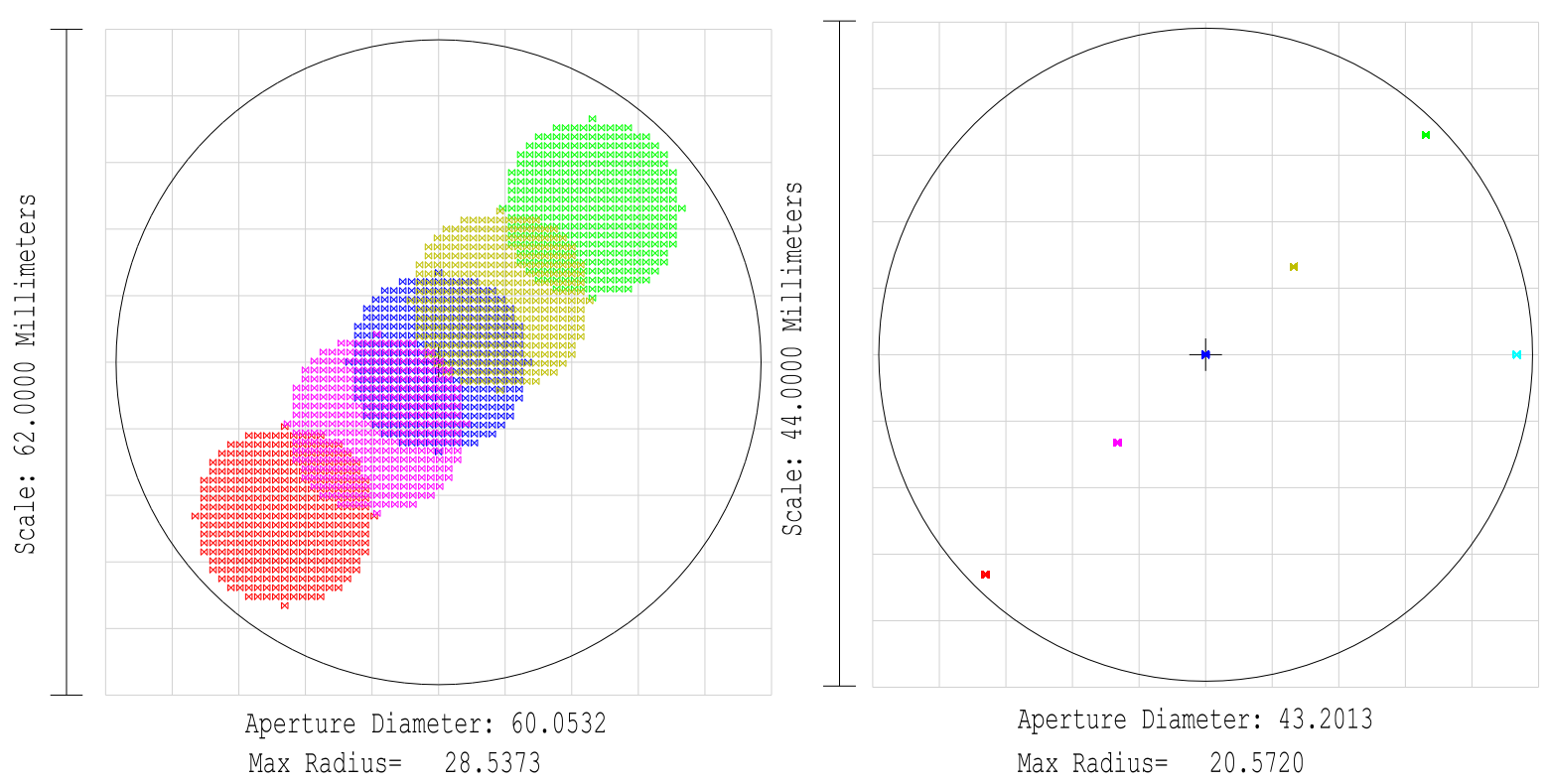

Figure 11.9.: Footprint diagrams of selected field points along the VLT optical path: at the position of the end of the Nasmyth adaptor flange (left) $250 \mathrm{~mm}$ before the focus, and at the telescope focal plane position (right). Note the different scales in the two figures.

The footprints mark the fan of rays originating from a single field point on the sky, and thus determine the surface areas illuminated by these point sources (naturally the size of the PSF in the focal plane). The size of the aperture along the optical path results from the field points with the largest deviation from the optical axis. Plotted are the center point (blue), the CRIRES FoV boundaries at $\pm 35.36^{\prime \prime}$ (red and green, respectively; thus the diagonals of a $50 \times 50^{\prime \prime} \mathrm{FoV}$ ), and $\pm 14.14^{\prime \prime}$ field angles $\left(10 \times 10^{\prime \prime} \mathrm{FoV}\right.$; magenta and dark green $)$. The clear aperture size required to maintain the CRIRES FoV is hence $\gtrsim 60 \mathrm{~mm}$ for any device to be inserted at the adapter flange end. The size of the telescope focal plane needed by CRIRES is $\gtrsim 41 \mathrm{~mm}$ in diameter.

Such an approach mitigates the lack of space around the Nasmyth focus by making use of the room above the optical bench. ${ }^{4}$

\section{The collimation problem}

Challenges for designing such an endavour arise mostly from two aspects: first, the location of the carriage is close to the telescope focus (in fact, the focal plane sits at the carriage's edge; cf. Sec. 11.2). This means that forcing the beam into collimation over an extended field size is a serious problem at this proximity to the focus (the academic case of a single point source can be treated by simplistic geometrical optics, but is of no help as CRIRES and its AO

\footnotetext{
${ }^{4}$ The Nasmyth platform where CRIRES is mounted on provides clearance up to the dome ceiling. "Although there is no explicit limit to the instrument height above the Nasmyth platform, instruments should not extend beyond 4 metres above the Nasmyth platform floor." (Rupprecht 2005). Except for the (removable) enclosure around the CRIRES warm optical bench (for light tightness), no obstructions are present above the bench. The dimensions above the bench are limited, rather, for reasons of mechanical stability and load.
} 


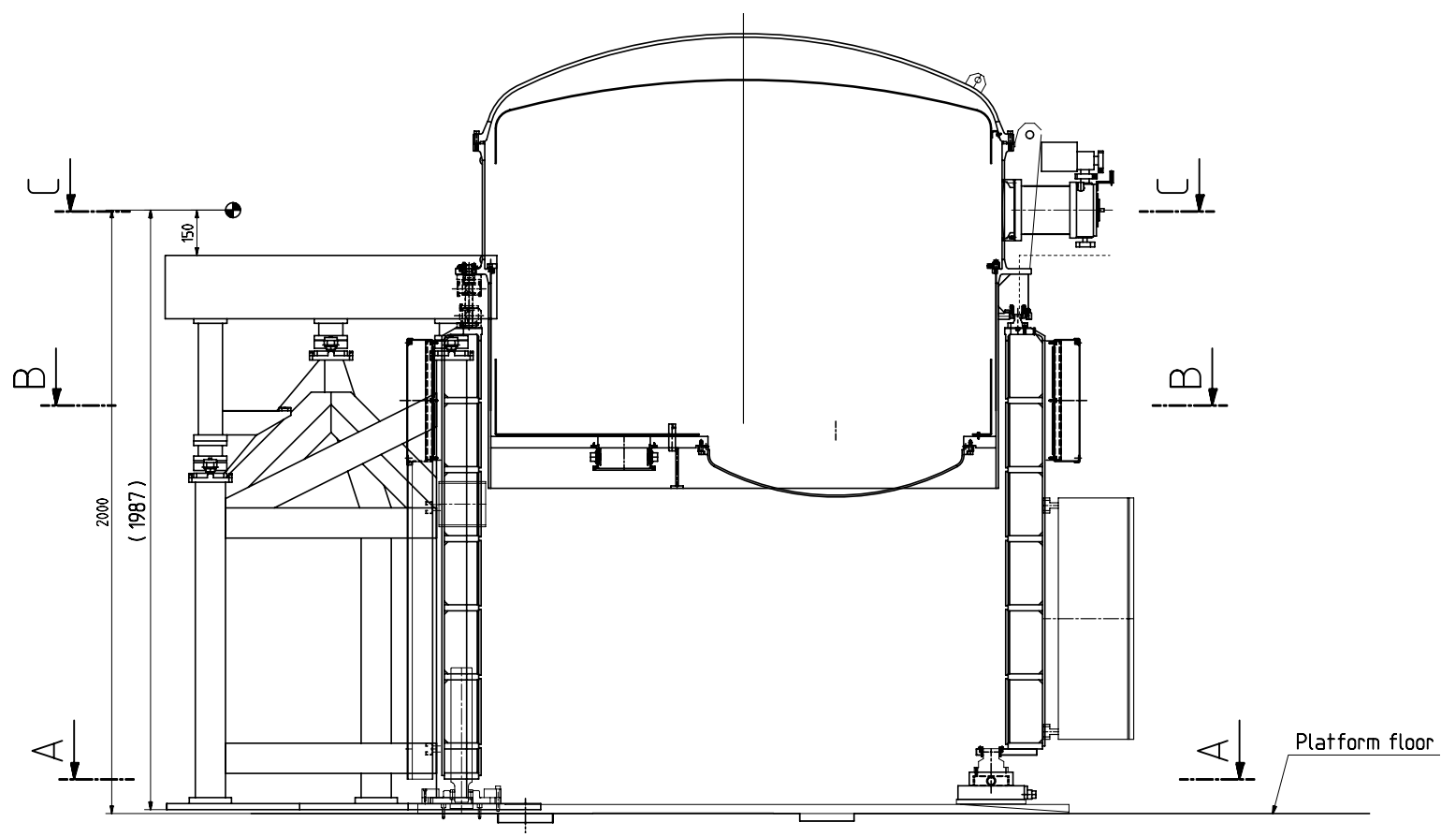

Figure 11.10.: Arrangement of CRIRES on the Nasmyth platform (section of drawing), seen in side view. The telescope adapter is to the left, the platform floor is on the bottom. The CRIRES vessel is mounted on an elevated structure to match the Nasmyth focus level. The warm optics (not shown) are accomodated on the optical bench to the left of the vessel, also on an elevated structure. (adapted from ESO/VLT-DWG-14500-0-300003).

require a spatially resolved field of view). An equally intricate problem is, second, to have restored the optical properties until immediately in front of the required focal plane. This is again severely hampered by the required field size, and by the inserted additional path-length for a gas cell mechanism (the latter being the reason for the entire exercise). The ultra-short distance left after the fold-back until the focal plane $(\mathrm{a}$ few $\mathrm{cm}$ ) renders this goal a fierce undertaking.

It is instructive to briefly discuss these difficulties in Fig. 11.11. Consider a converging telescope beam of a given focal ratio, shown in ray-tracings in Fig. 11.11 as the first surface. This beam is brought to focus (top panel), represented in three field angles (on-axis in blue, minimum and maximum field sizes in red and green, respectively). Closely behind the focal plane, an ideal (single) collimation mirror is inserted to produce a collimated beam for every field point (second surface, top panel). The collimated beam can be used to bridge a longer distance, eg. travel through a gas cell tube as in the case discussed in Sec. 11.1. However, the further off-axis the field points are, the larger the inclination between the collimated ray-fans of off-axis field points to the on-axis ray-fan (ie. angular magnification). As a result, the aperture needed by the succeeding re-focusing mirror (third surface) quickly grows with the length of the collimation line.

Now consider the same setup, but with the collimator (second surface) moved to before the telescope focal plane with respectto the first case (middle panel in Fig. 11.11). Again, the $\mathrm{RoC}$ is chosen so that the beam is collimated, and brought back to focus by another mirror 

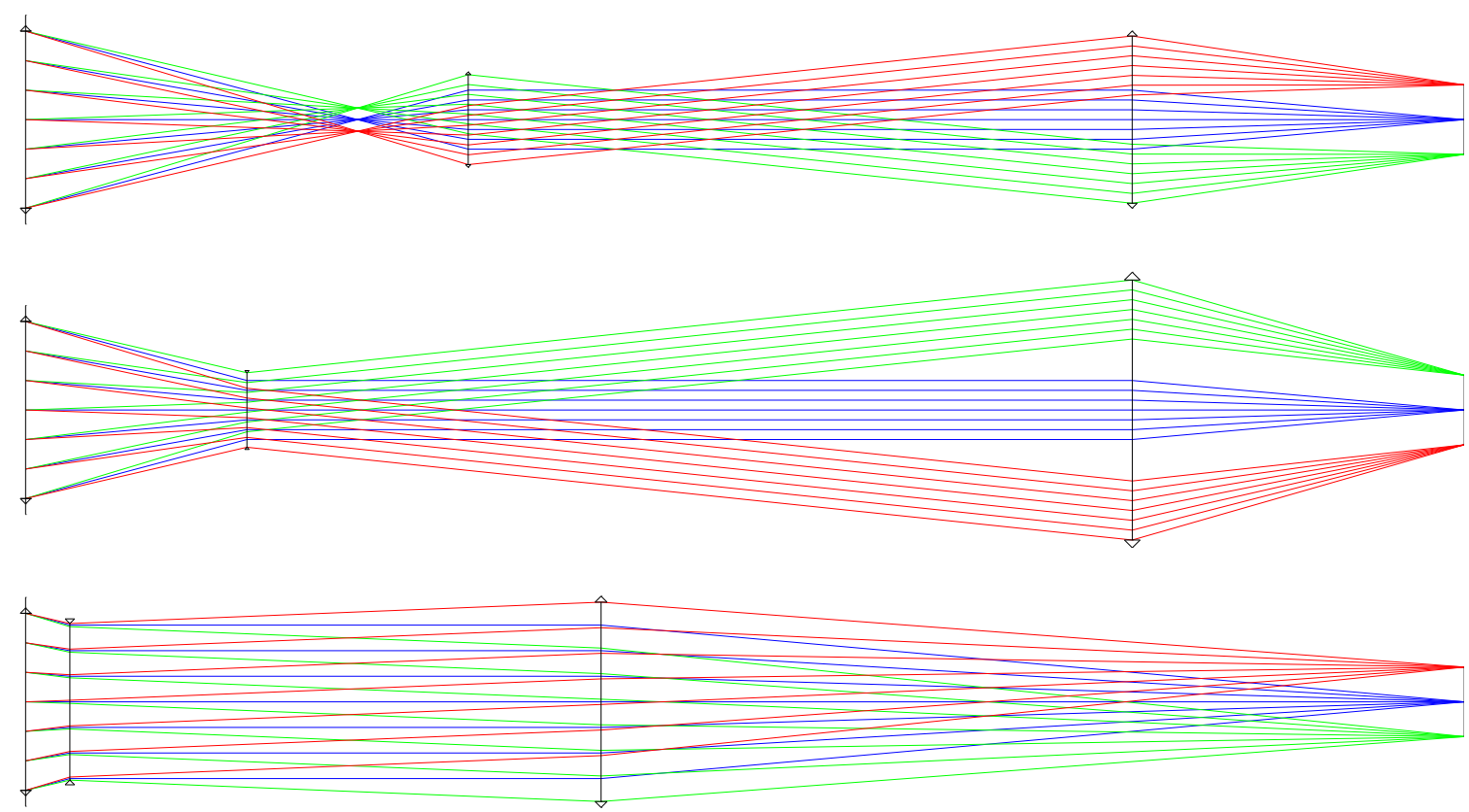

Figure 11.11.: Idealistic ray-tracings to illustrate the collimation problem. The collimator (second surface) is placed behind the telescope focus (top panel), in front of that focus (middle panel), and far in front (lower panel). A converging beam for three field angles (on-axis in blue, \pm off-axis in green and red, respectively) serves as input (leftmost surface), eg. a telescope. The exit focal ratio is preserved in all three cases, but at different angular magnifications. For details, see text.

(third surface). The resulting angular separation of the field points relative to the on-axis ray-fan is the same, compared to the upper panel ${ }^{5}$. However, due to the longer collimation line, the aperture for the re-focuser further increases. This, in turn, means that the incident ray-fan angle on the image plane is also enlarged, ie. the angular magnification is larger than unity. Any optics following that image plane must accept such a large acceptance angle and the respective magnification - this is usually not the case. An existing setup such as CRIRES cannot cope with such a setup. Note that the focal ratio incident onto the image plane (fourth surface) is invariant in Fig. 11.11.

Finally, the lower panel in Fig. 11.11 shows the system with the collimator significantly moved away from the telescope focal plane ${ }^{6}$, towards an exit pupil image of the telescope. This resembles the usual way to employ a collimator. It keeps the following aperture small (re-focuser) while allowing for a long collimation line, and yields an incident angle on the image plane close to zero. But it requires physical space, to

1. place the collimator away from the telescope focus, to

2. enable a (long) collimation line, and

\footnotetext{
${ }^{5}$ This is true if the collimator has the same distance to the focal plane as in the upper panel of Fig. 11.11.

${ }^{6}$ Moving the collimator much behind the focal plane yields the equivalent same setup.
} 
3. to allow for the re-focuser to re-establish the input focal ratio.

Thus, ideally a collimator is placed into or near a pupil image to avoid the beam expansion illustrated in Fig. 11.11 (top and middle). In the case of CRIRES this physical space is not available, so that field expansion and the corresponding aberrations, and large incident angles have to be dealt with. For simplicity, and to illustrate the fundamental concept, the examples in Fig. 11.11 are paraxial ray-tracings, and neglect the physical extent of each beam towards off-axis. In reality, a multitude of optical aberrations for anything but the on-axis chief-ray come into play and complicate the setup (and have to be suppressed). A careful raytracing modelling is mandatory to cope with the situation and the boundary conditions. I will detail these issues in the following sections, and present ways to solve them.

\subsubsection{First order concept}

In preparation of what follows in the next sections, I consider the basic ideas discussed on Fig. 11.11 and apply them to the real conditions found for Crires. The VLT model serves to simulate the optical properties of the Nasmyth focus, according to the prescription in Secs. 11.2.2 and 11.2.5. The aim at this point is to design a simple collimation mechanism, with the following assumptions:

1. The spectrograph (CRIRES) can be re-located.

2. The design does not need to meet the other requirements elaborated on in Sec. 11.2. In particular, this concerns the presence of the warm pre-optics, the size limitations by the calibration slide, etc.

The sole purpose is to move the Nasmyth focus further away from the telescope. For simplicity, the focus shall be moved along the optical axis, and mirror reflections are treated unsigned $^{7}$. The principles to be demonstrated in this exercise will become instructive in the later sections.

Fig. 11.12 shows a ray-traycing, based on the VLT-model, from the Nasmyth adapter flange to the focal plane, for an on-axis (blue) field and $\pm 14.14^{\prime \prime}$ and $\pm 35.35^{\prime \prime}$ off-axis diagonal field points (red and green; magenta and olive, respectively). I place a parabolic collimator at the beginning of the CRIRES calibration carriage, insert a collimation line of length $500 \mathrm{~mm}$, re-focus by a second parabolic mirror, and continue with the un-altered $180 \mathrm{~mm}$ until the focus. Fig. 11.13 (top) shows the resulting layout, when the RoC is tuned to the properties of the on-axis field. This yields a perfect restoration of the initial setup, with the difference of a seamlessly inserted $50 \mathrm{~mm}$ length. However, for the off-axis ray-fans this translates into a significant deflection (while being collimated), so that strong angular magnification results in Fig. 11.13 (bottom). The fundamental problem is the same as stated in Fig. 11.11 in the previous section. Note that these calculations are done for the limited FoV of $50^{\prime \prime} \times 50^{\prime \prime}$, not for the nominal FoV of the VLT $\left(30^{\prime}\right)$.

Next, I optimize the (re-)collimation mirrors not only for the on-axis field, but I try to find a solution that brings all fields considered (on and off-axis) to collimation ${ }^{8}$. Obviously,

\footnotetext{
${ }^{7}$ Since a mirror surface changes the sign of the propagating rays, the mirror must a) be operated in a tilted way or off-axis if the reflected beam is desired to not hit the preceeding elements; or b) the preceeding element/mirror must have an appropriate opening (eg. hole) to allow for the beam to pass. The sign of propagation is neglected here for simplicity, as if the mirrors were "transparent". Lenses could alternatively be employed, but would not do justice to the simplified treatment desired for the moment, as additional effects come into play (chromatism, spectral transmission).

${ }^{8}$ In the following, the term "collimation" does not imply perfect collimation.
} 


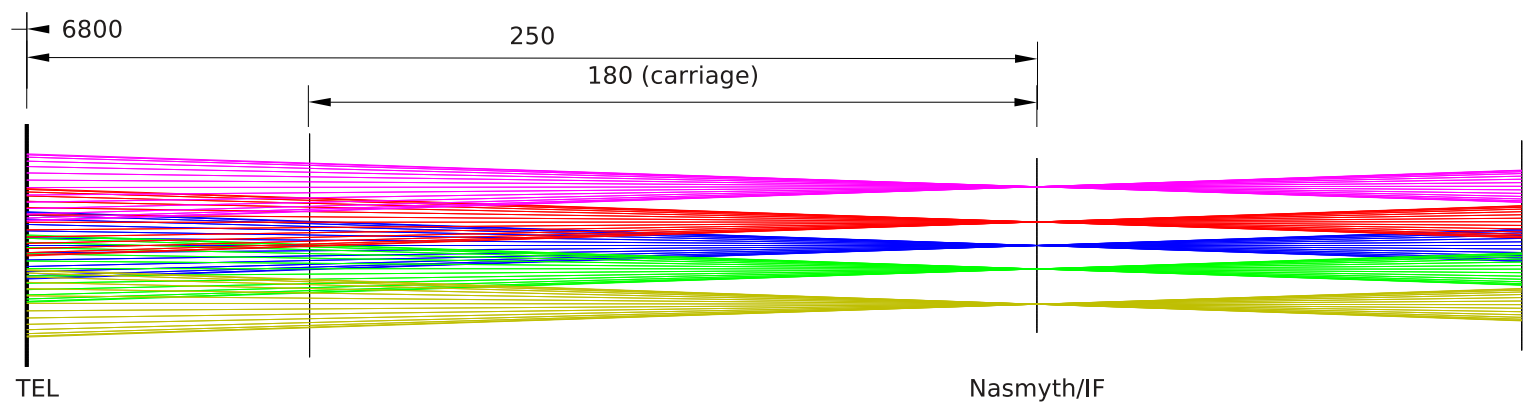

Figure 11.12.: Optical layout of the VuT Nasmyth focus area, from the end of the adaptor flange (TEL) to the focus (Nasmyth/IF). On-axis (blue) and $\pm 14.14^{\prime \prime}$ and $\pm 35.35^{\prime \prime}$ off-axis diagonal field points (red and green; magenta and olive, respectively) are shown.

at this location of the optical train, this is physically not feasible, for the reasons discussed above (proximity to the f/15 telescope focus). The best compromise results in Fig. 11.14. For clarity, the on-axis layout (blue, top panel) is shown separately. Note that the length of the carriage remains fixed. Now, the angular magnification is reduced to a negligible minimum (ie. nearly unit angular magnification), but the resulting focal ratio drops to $f / 4.4$. It is important to realize that the collimator cannot maintain the focal ratio if we strive for unit angular magnification, while the length parameters are fixed (collimator length and back-focal distance).

In a third step, I do not fix the back-focal distance, but keep it as a free parameter (together with the mirrors' curvatures). The system is supposed to yield good collimation, while maintaining the $\mathrm{f} / 15$ and unit angular magnification. A solution now exists as the problem is not under-determined anymore, and requires the back-focal distance to grow to some $600 \mathrm{~mm}$ for compensation (Fig. 11.15). Thus, the fundamental problem for a collimator to work is the need for compensator space. This (conceptual) solution would mean for CRIRES to insert an additional space of $\sim 1.1 \mathrm{~m}$ along the optical path between focus and instrument. Such extra space is not available (see the requirements in Sec. 11.2). Hence, for a working concept of a CRIRES long-path cell device, the trade-off process to find a feasible solution has to mitigate the demand for compensator space.

\subsubsection{CRIRES model}

The optical parameters of CRIRES are described in Delabre et al. (2000). For the purpose of designing a long-path gas cell mechanism, a model of the entire instrument is not needed. As the input to the spectrograph shall remain unchanged, an analysis of the input parameters can be performed at the instrument focus, and before (cf. Figs. 11.5 and 11.6). Thus, to evaluate the specifications, the optical model of the cell mechanism must reflect the CRIRES warm optics modules. In particular, the AO module needs a proper description to meet the pupil position requirement. The derotator module is included for completeness. It is not strictly needed for the modelling process, as it only involves three (tilted) flat mirrors in the diverging beam in front of the $\mathrm{AO}$ mirror. No optical powered elements are involved, so the only net effect the unit has is a change in effective optical path length and deviations in the optical path difference (OPD). 


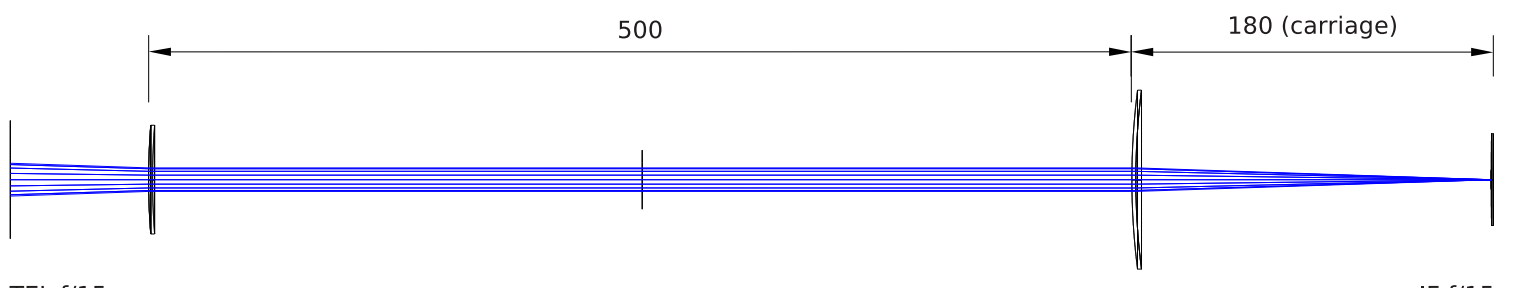

TEL $f / 15$

IF $f / 15$

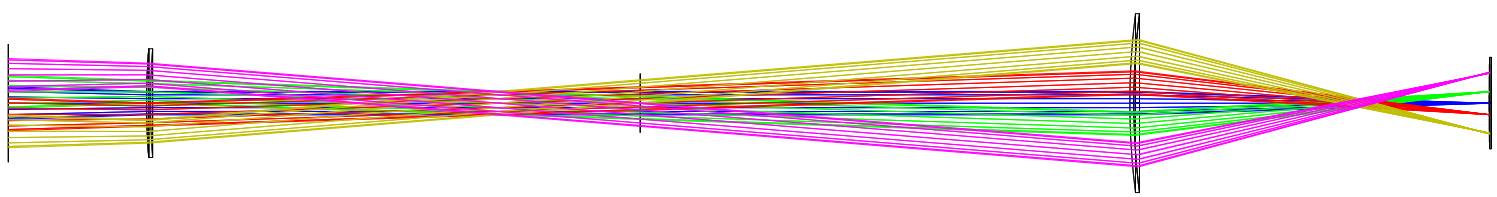

Figure 11.13.: Solution for a collimator with optimal collimation. The focal ratio is restored, but high angular magnification occurs. For clarity, the top panel shows the onaxis ray-fan separately, while the bottom panel plots both on-axis and off-axis fields. Colour coding as in Fig. 11.12.

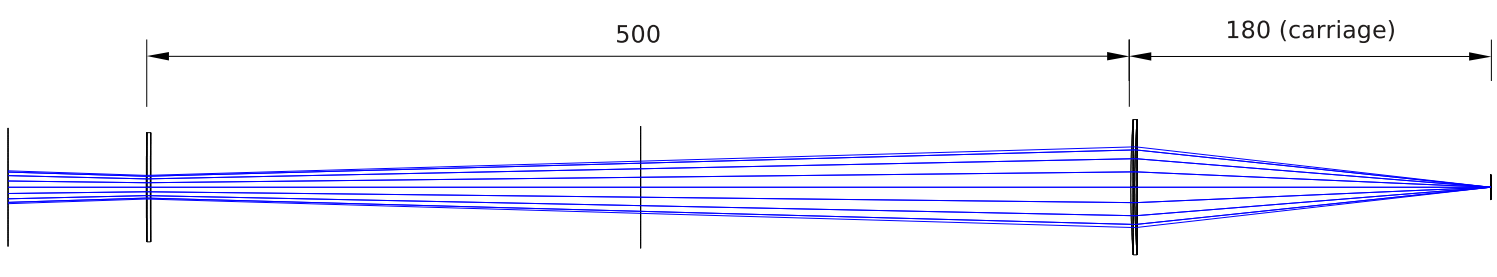

TEL f/15

IF $\mathrm{f} / 4.4$

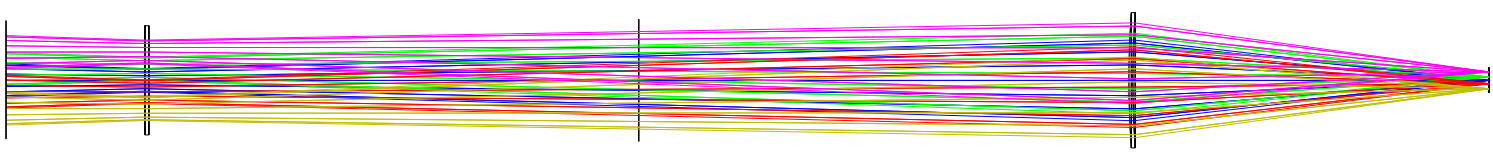

Figure 11.14.: Solution for a collimator with the back-focal distance as a fixed parameter. The angular magnification is negligible, but the focal ratio cannot be restored. For clarity, the top panel shows the on-axis ray-fan separately, while the bottom panel plots both on-axis and off-axis fields. Colour coding as in Fig. 11.12.

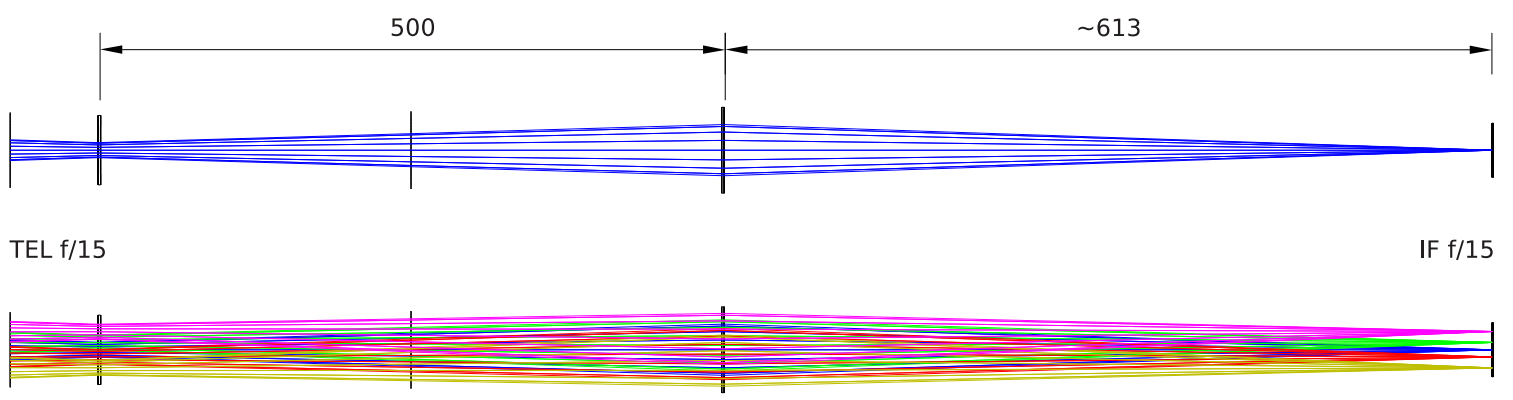

Figure 11.15.: Solution for a collimator with the back-focal distance as an additional free parameter. The focal ratio is restored, and angular magnification negligible. For clarity, the top panel shows the on-axis ray-fan separately, while the bottom panel plots both on-axis and off-axis fields. Colour coding as in Fig. 11.12. Note the different scale compared to the previous figures. 


\section{Adaptive optics module}

The CRIRES AO relay optics are specified in Table 11.2 following the prescription in Delabre et al. (2000). AOM2 serves to re-image the telescope pupil onto the deformable mirror (DM). To maintain this property with an intermediate gas cell mechanism, the AO relay module needs to be taken into account of the modelling process. Other parts of the AO system (wave front sensing unit, tip-tilt correction by $\mathrm{M} 2, \mathrm{AO}$ probing on the slitviewer) can be neglected. The AO relay model comprises four tilted mirrors, with respect to the optical path. It has unit magnification and relays the $\mathrm{f} / 15$ focal plane into the CRIRES cryostat. The normal shape of the DM can be considered flat. Note that the distance between the Nasmyth focus and AOM1 is given as the optical path, and thus has to account for the derotator. The physical distance is thus $719.17 \mathrm{~mm}$. The model is shown in Fig. 11.16.

\section{Derotator module}

The derotator provides control over the field rotation inherent to the Nasmyth, so that the orientation of the CRIRES slit is invariant on the sky. The derotator comprises an Abbe-König prism with the two external prism faces used in reflection. A flat mirror is placed in parallel to the incoming beam, to send the light back onto the second prism face. The general design is described in Delabre et al. (2000), but no details on dimensions and geometry are provided. It is concluded from the dimensions in Fig. 11.6 that the deflection angle on the prism is $105^{\circ}$, and the incident angle on the flat folding thus $15^{\circ}$. It is assumed in the following that these values be correct, as no further documentation is publicly availabe. The optical path length (OPL) of the derotator arms is then $180 \mathrm{~mm}$ each. The vertex of its first surface is located $50 \mathrm{~mm}$ behind the Nasmyth focus position. Fig. 11.16 shows the modelled derotator optical arrangement.

Table 11.2.: Optical design data for the AO relay optics used for the optical modelling. For details, refer to ESO technical report VLT-TRE-ESO-14500-2096 (Delabre et al. 2000).

\begin{tabular}{rccccl}
\hline \hline Surface & $\begin{array}{c}\text { RoC }^{a} \\
{[\mathrm{~mm}]}\end{array}$ & $\begin{array}{c}\text { Distance } \\
{[\mathrm{mm}]}\end{array}$ & $\begin{array}{c}\text { Diameter } \\
{[\mathrm{mm}]}\end{array}$ & $\begin{array}{c}\text { Tilt } \\
{\left[{ }^{\circ}\right]}\end{array}$ & Comment \\
\hline OBJ & -2089.6 & 986 & - & & Nasmyth focus f/15 \\
1 & 119600 & 938 & & 35 & AOM1 \\
2 & -1890 & 1014 & & 3.2 & AOM2 \\
3 & variable & 637 & 60 & 8 & deformable AO mirror ${ }^{c}$ \\
4 & -12700 & 230.1 & & 45 & AOM3 \\
IMA & & - & & 4.2 & AO relay focus, ie. instrument focus f/15 \\
\hline \hline
\end{tabular}

Notes.

(a) Radius of Curvature.

(b) Axial distance to next surface.

(c) The AO-DM is sized to allow for a $50^{\prime \prime} \times 50^{\prime \prime}$ field of view. 


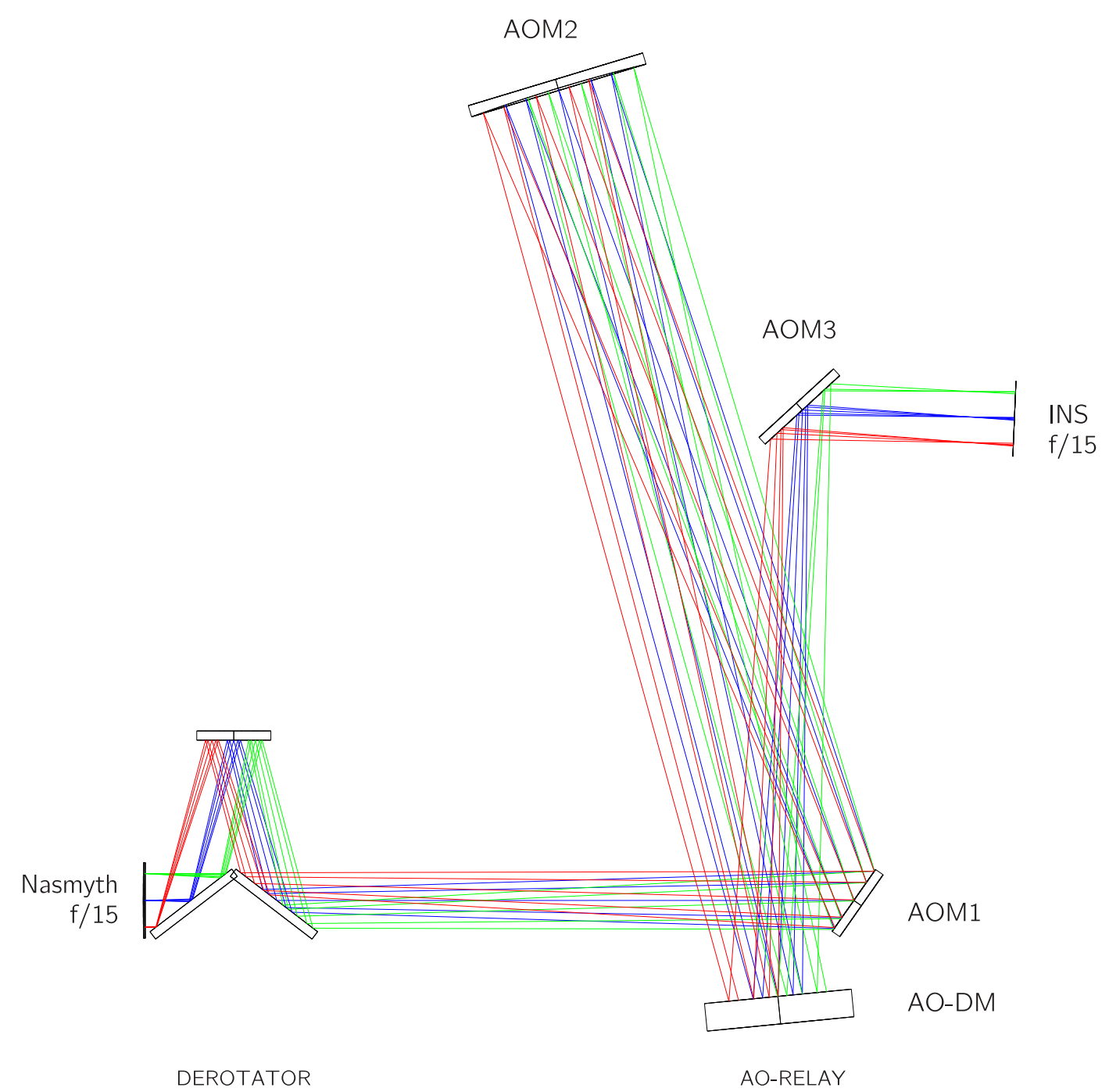

Figure 11.16.: Optical layout of the CRIRES warm optics modules relevant for the modelling process (derotator, left, and AO-relay, right). The derotator follows immediately after the telescope focus. The AO-relay re-images the $\mathrm{f} / 15$ focus into the instrument vessel (INS) at unit magnification, and places the telescope pupil onto the AO deformable mirror (AO-DM).

\section{Pre-slit optics module}

The CRIRES pre-slit optics (PSO) convert the input $\mathrm{f} / 15$ focus from the AO into an $\mathrm{f} / \sim 8$ beam onto the slit. This is done to match the focal ratio to the one of the main spectrograph units (collimator onto echelle, and camera), so that the TMA can be used both ways (Delabre et al. 2000). The PSO also produces a pupil image for placing a cold stop. The module is located after the dichroic entrance window to the spectrograph vessel.

As described above, the AO module employs spherical mirrors in tilted arrangements. This arrangement produces strong astigmatism, which is compensated for by the two further spherical mirrors AOM1 and AOM2. However, the compensation is effective only for the 
on-axis field point, but not off-axis. The purpose of the PSO module inside the cold CRIRES environment is also to correct for the field astigmatism caused by the AO. The pre-slit module comprises four spherical mirrors (see Table 11.3), also arranged at tilts, where the tilt angles are optimized as to compensate for the astigmatism.

In order to asses the image quality achieved at the slit (where it becomes apparent), the pre-slit module needs to be taken into account. My model of telescope and instrument thus has to incorporate this module to correctly reproduce the real optical performance of the entire optical train. Without the PSO, the modelling process of an additional optical device, striving for best image quality, would overcorrect the aberrations introduced by the AO, relative to the instrument focus. The real performance at the slit would then be degraded.

The PSO as given in Table 11.3 applies to the current CRIRES-setup in long-slit operations. The scheduled instrument upgrade (Follert et al. 2013), enabling cross-dispersion (and decommissioning the long-slit mode), features a severely modified PSO. In fact, the PSO will be completely replaced by a cross-disperser unit (CDU). As a result, the spectrograph entrance slit is relocated into the instrument focus (f/15), shortly behind the dichroic window. Due to the missing PSO, the upgrade implies a degradation of the image quality at the slit, suffering from strong astigmatism. The slit-viewer objectives and camera can partly correct for this (currently not foreseen), but obviously only at the level of the slit-viewer detector.

For the instrument model, I assume the CRIRES+ setup (Follert et al. 2013), ie. the slit positioned at the instrument focus, and not the current PSO. As a consequence, the cell concept can be optimized to deliver optical performance superior to the nominal CRIRES+ configuration.

However, for a realistic prescription, I incorporate into the model description the dichroic entrance window. This is beneficial as a tilted parallel plate causes further astigmatism, which the model can then compensate for. The window is tilted at $30^{\circ}$ with a $1^{\circ}$ wedge, and is comprised of $10 \mathrm{~mm}$ thick $\mathrm{CaF}_{2}$. Moreover, a strong chromatic effect results from the window, so that the slit location needs to be positioned depending on the wavelength of interest. The

Table 11.3.: Optical design data for the (current) cold pre-slit optics used for the optical modelling. For details, refer to ESO technical report VLT-TRE-ESO-14500-2096 (Delabre et al. 2000).

\begin{tabular}{rccccl}
\hline \hline Surface & $\begin{array}{c}\mathrm{RoC}^{a} \\
{[\mathrm{~mm}]}\end{array}$ & $\begin{array}{c}\text { Distance } \\
{[\mathrm{mm}]}\end{array}$ & $\begin{array}{c}\text { Diameter } \\
{[\mathrm{mm}]}\end{array}$ & $\begin{array}{c}\text { Tilt } \\
{\left[{ }^{\circ}\right]}\end{array}$ & Comment \\
\hline 0 & $-^{c}$ & $10+51.53$ & 50 & 30 & dichroic entrance window ${ }^{c}$ \\
OBJ & & 800.3 & - & & Instrument focus f/15 \\
2 & 975.0 & 429.5 & 140 & 6.5 & ROM1 \\
3 & 7275.0 & 291.0 & 84 & 37 & ROM2 \\
4 & & 186.0 & 25.5 & & cold stop \\
5 & 521.0 & 99.8 & 42 & 8.5 & ROM3 \\
6 & 1140.0 & 70.2 & $32 \times 27$ & 32.5 & ROM4 \\
IMA & & - & $50^{\prime \prime} \times 50^{\prime \prime}$ & & entrance slit $\left(40^{\prime \prime}\right.$ á $0 .^{\prime \prime} 2$ or $\left.0 .^{\prime \prime} 4\right)$ \\
\hline \hline
\end{tabular}

Notes.

(a) Radius of Curvature.

(b) Axial distance to next surface.

(c) The dichroic $\mathrm{CaF}_{2}$ window is wedged $\left(1^{\circ}\right)$ and $10 \mathrm{~mm}$ thick. 


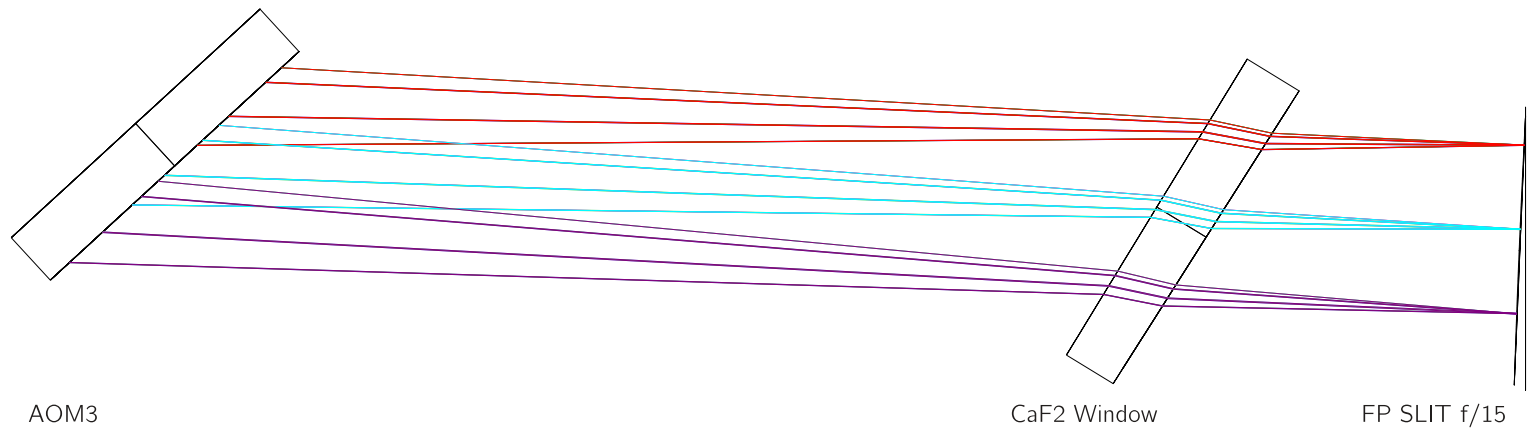

Figure 11.17.: Layout of the dichroic window, reflecting the light below $950 \mathrm{~nm}$ to the AOWFS, while longer wavelengths are transmitted into the spectrograph. The $\mathrm{CaF}_{2}$ window acts as a tilted parallel plate and produces astigmatism and chromatism. The window is tilted by $30^{\circ}$ (away from the AO-DM; cf. Fig. 11.6), the dichroic layer is on the first surface. The $1^{\circ}$ wedge is applied on the same side, with the sharper end towards the figure bottom (window edge closer to the AO-DM). Tilt and wedge are in the same plane. The image (righmost plane) is tilted by $2.4^{\circ}$, hence the entrance slit in the CRIRES+ config.

chromatism cannot be corrected without refractive elements. The $\mathrm{CaF}_{2}$ window has excellent transmission over the entire wavelength range of interest $(0.95-5.3 \mu \mathrm{m}) .{ }^{9}$ However, being the only refractive element along the optical train until the entrance slit, it is also the limiting bottleneck in terms of wavelength dependent disturbances. Fig. 11.17 shows the effect and layout of the dichroic.

\subsubsection{NAGICS system overview}

The novel absorption gas cell design for the CRIRES spectrograph (NAGICS) is a gas cell device designed to meet all requirements of the VLT (Sec. 11.2.3) and of the CRIRES spectrograph (Sec. 11.2), while at the same time it maximizes the available path length. NAGICs follows the design approach in Sec. 11.3, and is comprised of three sub-systems:

1. The fold-out (FO) sub-system provides two mirrors mounted on the CRIRES calibration train carriage. A standard slot on the movable carriage, which is also used for short-path gas cells and other calibration units, suffices to host the whole sub-system. A flat folding mirror (FM1) at $45^{\circ}$ intercepts the telescope beam coming from the Nasmyth adapter and redirects it perpendicularly to above the carriage. A second flat folding mirror (FM8) sends the converging beam coming from atop the carriage at $45^{\circ}$ to the nominal Nasmyth focus position. The unit thus only serves as a re-direction mechanism if the slide is in position. If the carriage train is moved out of the optical path, no interception occurs and the NAGICS is "deactivated".

2. A delay-line (DL) sub-system compensates for optical path differences (OPD) and assures that symmetries are not broken. This is achieved by a set of folding mirrors (FM2-FM5) that are optically null-powered, and all operated at $45^{\circ}$.

\footnotetext{
${ }^{9}$ Wavelength dependent refractive indices $(0.2-12 \mu \mathrm{m})$ and transmission values $(0.3-10 \mu \mathrm{m})$ are used for IR grade $\mathrm{CaF}_{2}$ from ISPoptics; www.ispoptics.com.
} 


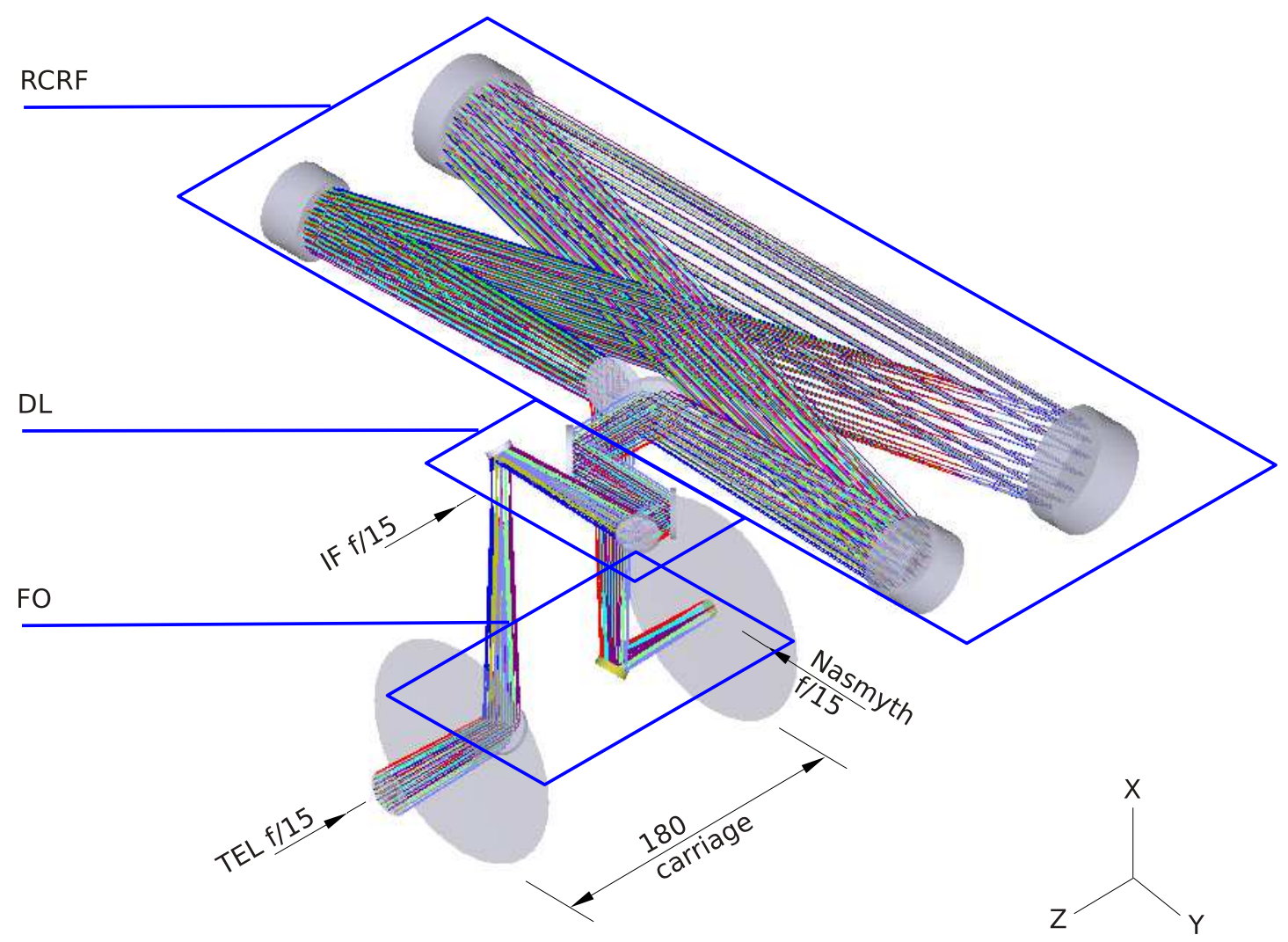

Figure 11.18.: NAGICS optical model rendering. The system is mounted on two different levels. The fold-out (FO) sub-system is located on the calibration carriage, in the Nasmyth focus' plane. The delay line (DL) and the re-collimation and re-focuser sub-system (RCRF) are mounted on a separate, elevated bench, together with the gas containing vessel (not shown). The telescope beam properties at the Nasmyth focal plane remain unchanged with the FO in place or out. See Fig. 11.19 for labels.

3. The re-collimation and re-focuser sub-system (RCRF) hosts the actual gas cell volume, ie. enables the path length through the gas. The RCRF defines the geometry of the gas containing vessel and is governed by two principles: a) it must keep the beam diameter small to keep control over the overall vessel dimensions, and conversely b) it must maximize the path length enclosed by the gas vessel.

A model rendering of the NAGICS system is shown in Fig. 11.18, also indicating the subsystems. In Fig. 11.19, a topview drawing is presented with the optical elements labelled.

\subsubsection{Optical design}

The design concept of the NAGICS system strives to maximize the usable optical path in a gas containing vessel, while at the same time meeting the requirements. A fundamental design idea is to conceive a scalable concept, in which the cell length $L$ (see Fig. 11.19) can be chosen freely (within limits). This means that $L$ can be tailored to specific applications, so that the system can be tuned to deliver the desired OPL within a gas of choice. Once a length $L$ is 


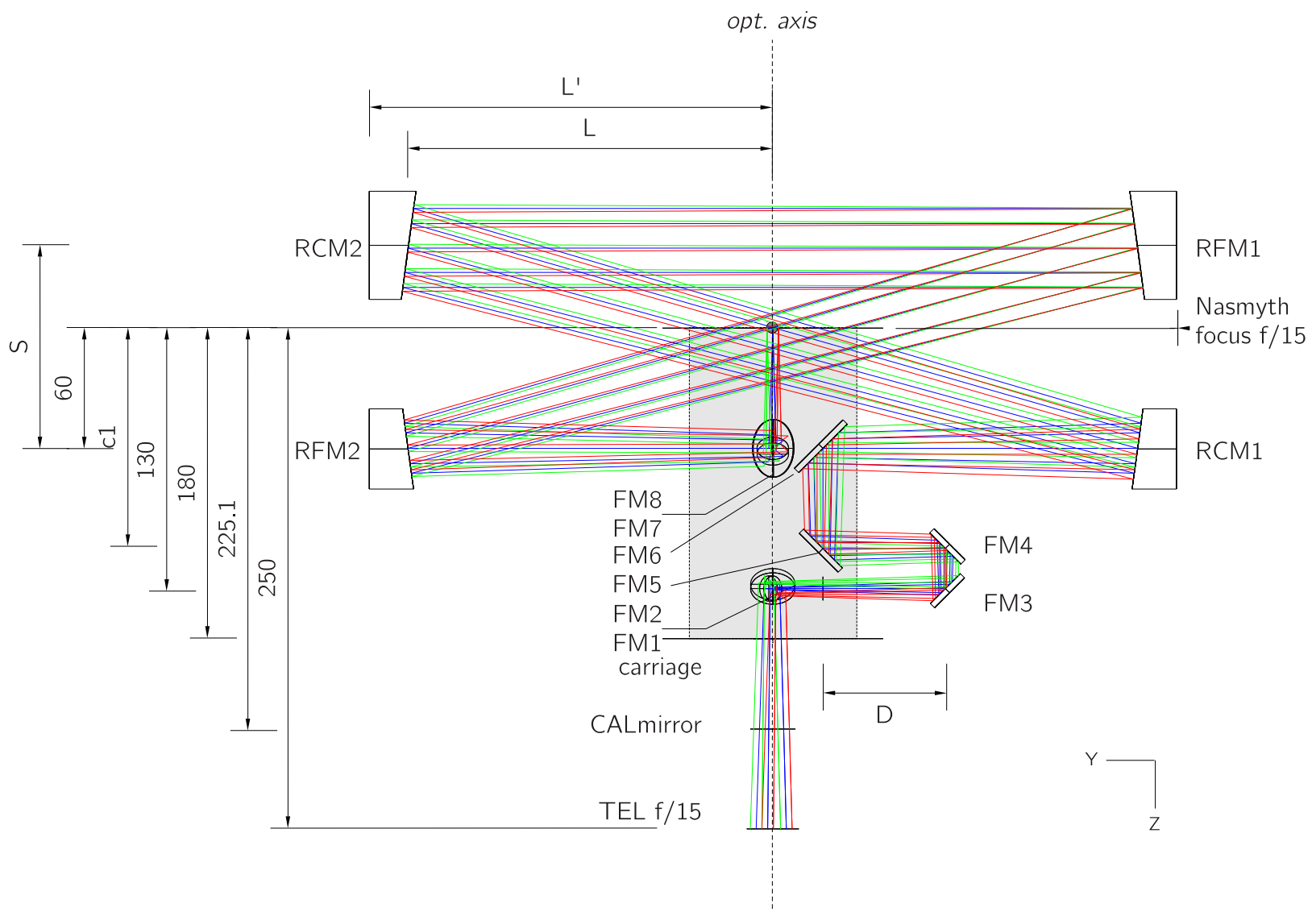

Figure 11.19.: Optical layout of the NAGICS concept (top view). The telescope beam exits the Nasmyth adaptor at the figure bottom, and is redirected to an elevated, upper plane by the fold-out (FO) unit (mirror FM1). A delay line (DL) unit keeps the optical path length (OPL) symmetric (FM2-FM5), followed by the system to re-collimate and to re-focus the beam (RCRF, usable for traversing a calibration gas). The converging $\mathrm{f} / 15$ beam is sent downwards again to the lower level (FM7) and fed to the nominal focal plane with restored properties (by FM8), and subsequently to the CRIRES warm optics. FM1 and FM8 are mounted on a carriage train that can be moved out of the beam (indicated by theg shaded area), with the same beam properties arriving at the Nasmyth focal plane for either position of the train carriage. The geometry of the cell mechanism is defined by the DL length parameter $D$, the OPL parameter $L$, and the arm separation parameter $S$, where $D, L, S$ depend on each other. The geometry can be scaled to obtain eg. the desired OPL. The design shown here features an additional optical path length (OPL) of $1.5 \mathrm{~m}$ within the RCRF ( $L=200 \mathrm{~mm}$ ).

set, the model optimization process will try to find the best optical solution that fullfills the requirements. It will also adapt the length of the delay line $D$, and the mirror properties of all powered elements. Ultimately, the scalability is limited by four factors:

1. The height of the RCRF sub-system above the CRIRES warm-optics bench (AO-bench; see below). This separation between the two benches is defined by the extension in 
height of the elements on the AO-bench, and by the structure and support of the RCRF-bench. It is assumed that the derotator is the highest unit on the AO-bench with about $180 \mathrm{~mm}$ vertical extension atop the optical axis. Therefore, a bench separation $H=400 \mathrm{~mm}$ is baselined (measured between the interception of the optical axis on FM1 and FM2). The separation $H$ should be kept small, as a larger $H$ results in increasing beam diameters in the diverging beam, and so requires larger mirror sizes (which, in turn, tighten the space constraints between adjacent mirror mountings and structures). The mirror diameters also grow with increasing $L$, for the same reason. The NAGICs model compensates for changes in $H$, as they are required by the mechanical setup, during the optimization process.

2. The FoV. The size of the Nasmyth focal plane (FP) scales with the required field angles on the sky ( $\sim 582 \frac{\mu \mathrm{m}}{\prime \prime}$, field dependent due to FP curvature). All following apertures along the optical axis thus also scale with FoV (at different rates though), and larger clearance is required to avoid obstruction of the beam for larger FoV. As an example, the folding mirror that suffers most from the FoV parameter, as its location is the farthest in the diverging beam before re-collimation by the RCRF, is FM6 (see Fig. 11.19). Its minimum required aperture increases from $33 \mathrm{~mm}$ to $131 \mathrm{~mm}$ in diameter for a FoV of $10^{\prime \prime} \times 10^{\prime \prime}$ compared to $100^{\prime \prime} \times 100^{\prime \prime}$, respectively, with implications on construction feasibility, obstruction of the beam (reflected by the first re-collimating mirror; RCM1), and interference with other units (FM5 in this case).

A larger FoV also translates in more severe optical aberrations by the powered surfaces (also see below), thus ultimately in decreased overall image quality.

3. The allowances for the mirror sizes. The largest apertures are required on the powered re-collimating- and re-focusing mirrors (RCM2 and RFM1). Implications of growing size concern, first, the space needed for the mirror assemblies, ie. including their housing and mounting structures. If the OPL of the system increases, the RCRF apertures must be enlarged to accomodate the beam diameter, so the separation between RCM1 and RFM1 (and equally RCM2 and RFM2) shrinks. At a threshold $L$, this separation becomes unfeasible for mounting, and additionally vignetting may occur by FM6 as with growing $L$ the angle of incidence $\alpha$ on eg. RCM1 (of the opical axis with respect to the surface normal) gets smaller (see Fig. 11.19). The separation is controlled by the design parameter $S$, which compensates for the above effect.

Second, aperture size concerns the cost of manufacturing and procurement (particularly if for the powered mirrors specialized shapes are chosen; see below).

4. Optical aberrations. The RCRF arm separation $S$ (hence, $\alpha$ ) is to be kept small to keep the overall design compact, and to keep optical aberrations low. The latter increase in amplitude, the further off-axis the RCRF mirrors are operated (the NAGICS optimization process is tailored in a way to best-cancel out aberrations).

I discuss each sub-system in the following, and describe its properties and workings.

\section{Fold-out sub-system}

The FO sub-system is summarized in Fig. 11.20. It is defined by the position of FM1 and FM8 with respect to the carriage. The size of the mirrors is constrained by the footprints of the maximum allowed field angles at these positions. Given the proximity of the normal Nasmyth 
focus, located on the edge of the carriage facing the instrument, the beam diameter on FM1 (its center positioned at $130 \mathrm{~mm}$ in front of the FP) for the full FoV $\left(50 \times 50^{\prime \prime}\right)$ amounts to $60 \mathrm{~mm}$ (including the $45^{\circ}$ projection effect by the FM1 tilt). This aperture size also fits FM8, where the beam size has further narrowed, at a position $60 \mathrm{~mm}$ before the FP. The latter distance to the FP is kept as large as possible, as to avoid reflecting surfaces very close to the FP where impurities on the surface immediately degrade reflectance and image quality, due to the small beam spot sizes. The original FP is transfered into an intermediate focus (IMF) by FM1, $20 \mathrm{~mm}$ below the upper folding mirror in this particular configuration. The IMF can be used, if necessary, to place a field mask in order to filter for a reduced FoV and block straylight (cf. Sec. 12.3.1). Instead of individually mounted FM1 and FM8, a prism can be employed with $45^{\circ}$ wedged faces used in external reflection. This eases mirror alignment of the FO and increases stability, and can easily be mounted on the sliding carriage.

\section{Delay-line sub-system}

The DL is introduced for reasons of symmetry (Fig. 11.21). Given the immutable location of Nasmyth FP and calibration carriage clearance, the following problem needs to be solved: the FO only changes the location of the Nasmyth FP, but not its relative distance from FM1 (or the beginning of the carriage). At the other end, the beam is brought back onto the original optical axis by the FO sub-system (FM8), so that the FP is to be restored at its original place. However, at the starting end there is now an intermediate FP atop of the FO (the flipped original Nasmyth FP), while at the exit end of the FO there is no such intermediate FP to the top. The relative OPL is thus different from the intermediate focal plane at the starting end of the system (which becomes the normal Nasmyth FP once the carriage is moved out, see Fig. 11.20) to the entrance of the RCRF (in the plane of the Nasmyth optical axis) than the OPL from the RCRF exit (also in the plane of the Nasmyth optical axis) to the re-imaged (restored) Nasmyth FP. This OPL is longer on the exit side than on the entrance side, and is a result from the space constraints at the Nasmyth, cf. Sec. 11.2.3.

To restore symmetry, some extra OPL is needed before entering the RCRF, depending on the length parameter $L$. This is to resolve the competing effects outlined in Sec. 11.1 when re-collimating and re-focusing, and enables to restore the beam at f/ 15 without angular magnification. The symmetry also enables cancellation of optical aberrations, thus improving the attained image quality. The DL compensation is slightly disturbed by the competing requirement to image the telescope pupil onto the AO-DM, so that the NAGICS optimization process also has to solve for $D$ to find the best overall solution. The parameters $C_{i}(i=$ $1 \ldots 5)$ are fixed as needed for mounting clearance and to avoid vignetting, depending on the configuration.

\section{Re-collimation and re-focuser sub-system}

The RCRF is the NAGICS analogue of a simple collimator line outlined in Sec. 11.1. It comprises two arms, where the first collimates the incoming light (entering at FM6, see Fig. 11.22) by a combination of two concave mirrors RCM1 and RCM2. These are operated in off-axis mode to ensure the necessary deflection angle (related to the separation parameter $S$ ), and their shape is primarily governed by the need to achieve collimation over the entire allowed FoV. Note that only in the theoretical case of a single on-axis point source, a single parabolic mirror would suffice as a collimator. The shortening of the OPL in this arm (due to the position of FM6) needs to be compensated by the DL. 


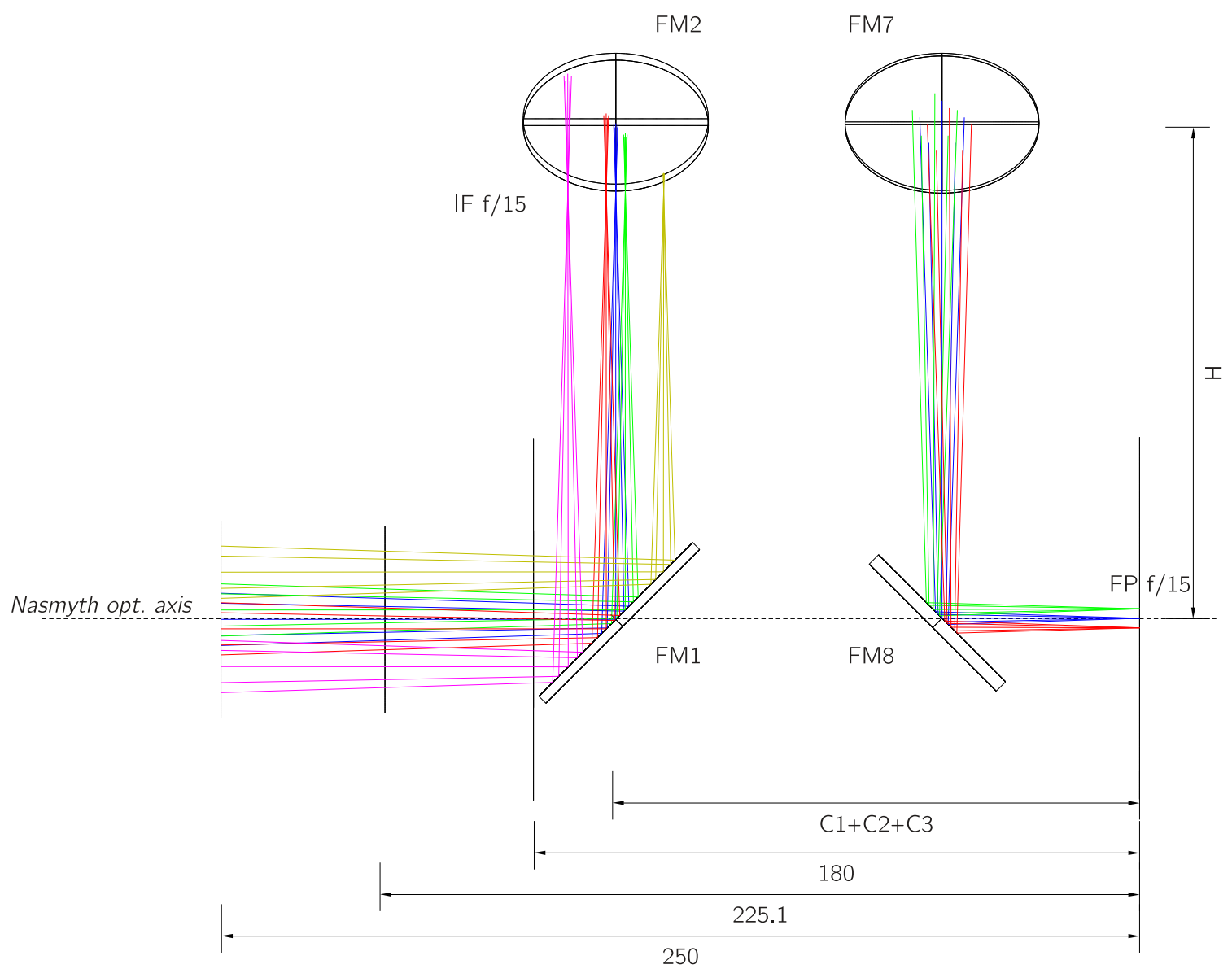

Figure 11.20.: Fold-out (FO) sub-system in side view. The FO serves to redirect the telescope beam (entering from left) out of the Nasmyth plane (with respect to the platform) by means of a flat $45^{\circ}$ mirror (FM1; left). Above FM1, the delayline sub-system begins. Leaving the RCRF sub-system downwards, the beam is restored into the Nasmyth plane by a second fold mirror FM8 (right), before the focal plane. Note that due to multiple foldings within the NAGICs, the orientation of the focal plane is flipped relative to without NAGICS in place. Also note that due to FM1, the FP becomes a flipped intermediate focus close to the upper folding mirror (20 $\mathrm{mm}$ in front, in this configuration).

Collimation is then reversed by the second arm, in which RFM1 and RFM2 re-image the field to the specified FP. The FP is located at the very same position as the nominal Nasmyth FP without NAGICS, below the RCRF. For this purpose, the light is folded out of the RCRF by the flat exit-mirror FM7 ( $45^{\circ}$ tilted against the figure plane), and send downwards to the FO again. The combination of shapes for all curved mirrors is computed to attain best recollimation of the diverging beam for the full FoV near the symmetry axis (Nasmyth optical axis), image quality at the FP, and to provide the required beam properties and FP position, for a given length parameter $L$. A competing factor is that a telescope pupil image must be placed on the AO-DM, so the mirror curvatures also need to account for the corresponding imaging. The performance of different surface types will be further discussed in Sec. 13.1.2. The configuration shown in Fig. 11.22 features $L=200 \mathrm{~mm}, S=100 \mathrm{~mm}$. This guides the 


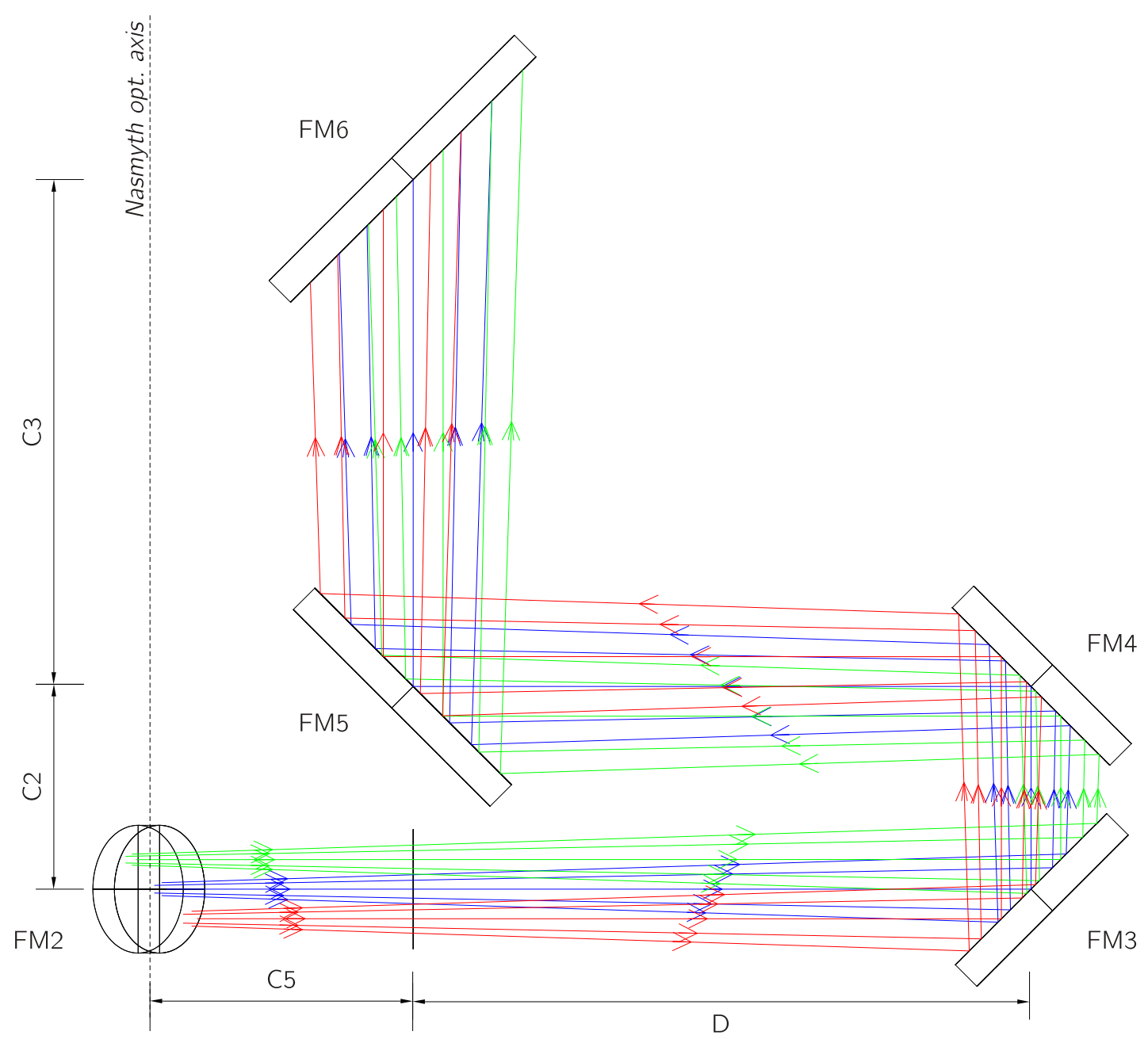

Figure 11.21.: Delay-line (DL) sub-system in top view. The DL restores a symmetric OPL with respect to the intermediate focus (IF), just below the tilted folding mirror (FM2, bottom left), and to the Nasmyth FP (at its nominal location, see Fig. 11.19). A symmetric, equally distant OPL between IF and FP relative to the RCRF axis is needed to maintain the optical beam properties, suppress aberrations, and obtain superb image quality at the FP. The DL comprises the folding mirrors FM2-FM5, all flat $45^{\circ}$ tilted (FM2 is tilted against the figure plane). The delay length $D$ is adjusted to match the OPL parameter $L$ (within the RCRF) and adapted to the spacing $C_{2}, C_{3}$, and $C_{5}$ between the subsequent fold mirrors (dependent on eg. the FoV allowances).

required mirror apertures of RCM2 and RFM1 to be $60 \mathrm{~mm}$ in diameter for the full $50^{\prime \prime} \times 50^{\prime \prime}$ Crires FoV (46 mm for a reduced FoV of $10 \times 10^{\prime \prime}$ ).

\subsubsection{Gas vessel design}

So far, I have explored the NAGICS concept considering only the optical mechanism that allows for the desired lengthening of the OPL. The purpose of the extra OPL, in the first 


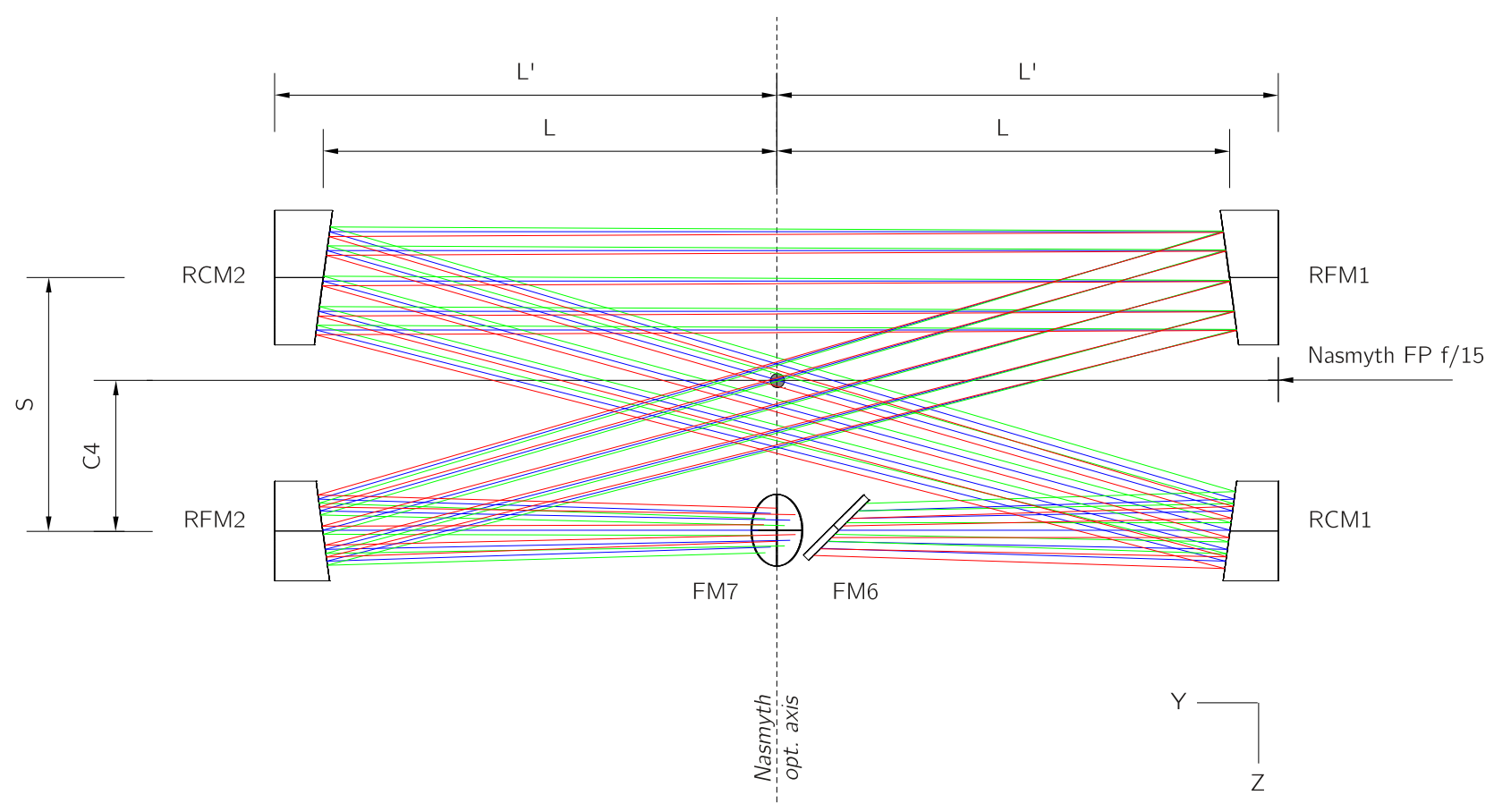

Figure 11.22.: Re-collimation and re-focuser sub-system (RCRF) in top view. The recollimator arm comprises FM6 (where the beam from the DL is injected), RCM1, and RCM2. The latter two are off-axis parabolics (in the simplest configuration) or more complex concave surfaces. The shortening of the arm's OPL (at FM6) needs to be compensated by the DL.

The re-focusing arm, consisting of RFM1, RFM2, and the exit-mirror FM7, reverses the collimation and enforces the focus at the specified, nominal location. All curved mirrors' shapes are computed to attain best re-collimation of the diverging beam for the full FoV near the symmetry axis (Nasmyth optical axis), image quality at the $\mathrm{FP}$, and to provide the required beam properties and FP position, for a given $L . S$ has a lower limit due to vignetting, and $C_{4}$ is set by the calibration carriage geometry $\left(C_{4}=60 \mathrm{~mm}\right.$ in this configuration).

place, is to have the light from the astronomical source travel through a calibration gas, using the extra OPL. Thus, a gas containing vessel is required to enclose the gas under stable conditions. The telescope beam then must be fed through this vessel.

In principle, two general options are feasible to incorporate a gas-tight vessel into the NAGICS concept:

1. A container can be thought of that encloses the whole NAGICS system but the FO sub-system. Such a vessel, of appropriate size, then needs to house the optical bench on which the DL and RCRF sub-systems are mounted on. Such an approach simplifies the interfaces to couple the beam into and out of the vessel, as only two optically transmitting windows are needed. These windows can be conveniently located above the FO, before FM2 and behind FM7 in the optical train. Their required aperture diameters are well controlled and typically around $50 \mathrm{~mm}(20 \mathrm{~mm})$ for the $50^{\prime \prime} \times 50^{\prime \prime}$ $\left(10^{\prime \prime} \times 10^{\prime \prime}\right)$ CRIRES FoV, respectively (configuration with $H=300 \mathrm{~mm}$ ). The window 
material is required to allow for good visual and NIR transmittance (optical red required by $\mathrm{AO}$ ). Low-OH quartz windows (to not introduce hydroxyl spectral signatures) have proven applicability with high transmission that can be further improved by NIR antireflection (AR) coatings. Sapphire is an obvious alternative material.

The drawback with this concept is primarily the size and complexity of the vessel, especially if larger $L$ are desired. As the DL and RCRF, to be mounted on an optical bench for stability, must go inside the gas-tight vessel container, the latter must provide appropriate structures for stable mounting of the bench, and allow for the respective alignment. Application is limited to non-corrosive gases, that do not interact with neither the optical elements, their coatings, nor the structures and mounting materials. Furthermore, access to the optical components (be it maintance, alignment, or recoating) is hampered, and requires breaking the gas sealings. This means that gas cell conditions (pressure, gas, purity) change in case of opening and refilling. These factors render a full eclosure vessel rather impractical.

2. The captive gas vessel can be placed inside the RCRF sub-system, into the optical path between the mirrors. Such a vessel has a much reduced complexity, and resembles somewhat a scaled version of the existing tube-lilke gas cells. Given the proper dimensions (which primarily depend on $L$ and $S$ ), a tube-like structure can be mounted independently of the optical setup, and be placed and removed separately. This offers the great benefit to characterize, align, and (re-)fill the vessel without deteriorating the optical system. Furthermore, the vessel can be replaced by potential copies and/or refilled, so that the calibrator (captive gas combination) remains flexible. In this way, different calibrators can be easily operated to match the desired characteristics, ie. wavelength range (cf. the discussion in Chapter 10), just by insertion of the appropriate gas vessel (the path lengths can be varied, as long as the vessel windows can accept the beam properly).

For this concept, the critical components are the transmission windows. Like in the above concept, the windows for the first beam entrance and last exit (in the optical path before FM6 and behind FM7, respectively; cf. Fig. 11.22) are uncritical. Again, they are perpendicular to the beam (minimizing aberrations), and their apertures do not exceed $\sim 50 \mathrm{~mm}$. However, unlike in the previous concept, the situation becomes slightly more complex for the side windows of the vessel. Here, closely to the powered mirrors, light enters and exits through the windows both perpendicularly as well as under a tilted angle, due to the reflected beam. Optical aberrations resulting from this acceptance angle must be well controlled, and analyzed for a specific configuration. While this acceptance angle becomes smaller for larger cell lengths $L$ (thus less critical), the required minimum window apertures increase at the same time. For very long $L$, the window diameters may become unfeasible (for reasons of manufacture and cost). As an example, a side window diameter of $200 \mathrm{~mm}$ is needed for a cell half-length of $L=1000 \mathrm{~mm}$. Specialized mirror shapes are able to reduce the collimated beam diameter, to a certain extent. The maximum window (and mirror) sizes need to be traded against feasible separations $S$ (ie. acceptance angle) and the desired path length $L$ for a specific NAGICS configuration.

Another critical aspect for this option are transmission losses due to the multiple window traversals. Sec. 12.3.1 reiterates on this issue.

A conceptual illustration of an outside-vessel is depicted in Figs. 11.24-11.23. The basic 


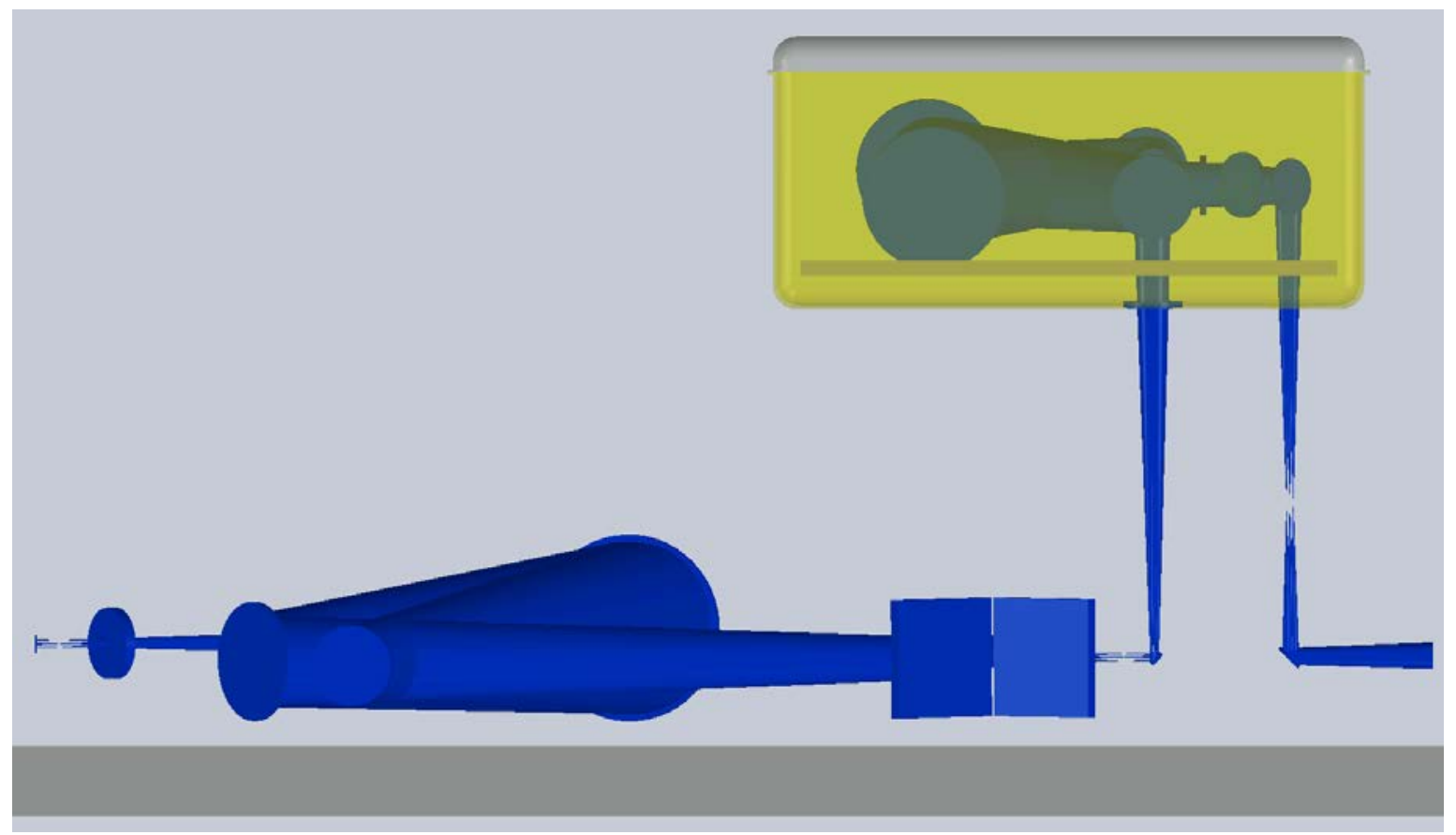

Figure 11.23.: 3D visualization of the gas container vessel concept enclosing the entire RCRF sub-system (side view). Image courtesy H. Anwand/IAG.

mechanical concept follows the guidelines given above. A detailed design and its tolerancing strongly depend on the exact NAGICS configuration to be selected, and need to be tailored accordingly. 


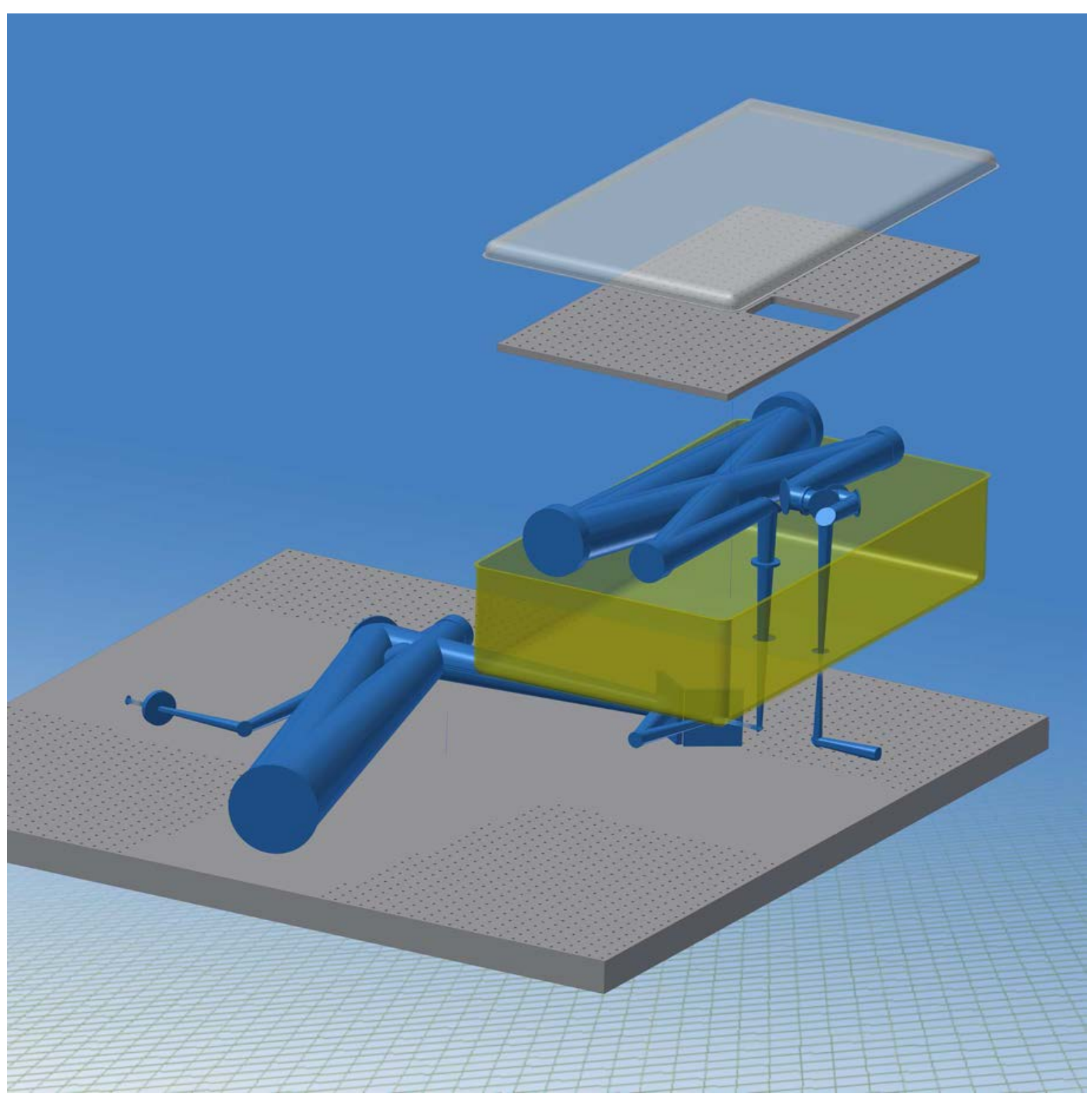

Figure 11.24.: 3D exploded view of the gas container vessel concept enclosing the entire RCRF sub-system. Image courtesy H. Anwand/IAG. 


\section{Performance Analysis}

\section{Contents}

12.1. Identifying a baseline design . . . . . . . . . . . . . . 140

12.1.1. Opto-mechanical assembly height . . . . . . . . . . . . . . . 140

12.1.2. Optical bench dimensions . . . . . . . . . . . . . . 141

12.1.3. Field of view . . . . . . . . . . . . . . . . . . . . . 142

12.1.4. Optical path length . . . . . . . . . . . . . . 142

Gas vessel dimensions . . . . . . . . . . . . . . . . . 144

12.1.5. Mirror properties . . . . . . . . . . . . . . . . . 144

Powered mirrors . . . . . . . . . . . . . . . . . . . . 144

Folding mirrors . . . . . . . . . . . . . . . . . . . . 144

Coatings . . . . . . . . . . . . . . . . 144

12.1.6. Baseline design parameters . . . . . . . . . . . . . . 145

12.2. Model performance verification . . . . . . . . . . . 145

12.2.1. VLT model at Nasmyth . . . . . . . . . . . . . . . . . 145

12.2.2. CRIRES model . . . . . . . . . . . . . . 146

Field dependent Strehl ratio . . . . . . . . . . . . . . . . . . . . . 147

Impact of the dichroic entrance window on Strehl . . . . . . . . . . . 148

Chromatic focal shift . . . . . . . . . . . . . . . . . . . 148

Encircled energy fractions . . . . . . . . . . . . . . 150

Spot sizes in the image plane . . . . . . . . . . . . . . . . . . . . 152

Pupil imaging on AO-DM . . . . . . . . . . . . . . . . 153

Reflectance and throughput . . . . . . . . . . . . . . . . 155

12.3. Baseline design performance analysis . . . . . . . . . 156

12.3.1. Optical performance . . . . . . . . . . . . . . 157

Field of view . . . . . . . . . . . . . . . . . . 158

Coating efficiency...................... 158

Image scale . . . . . . . . . . . . . . . . . . . . . . . 160

Field dependent Strehl ratios . . . . . . . . . . . . . . . 162

Chromatic focal shift . . . . . . . . . . . . . . . . 162

Encircled energy fractions . . . . . . . . . . . . . . . . 162

Spot sizes in the image plane . . . . . . . . . . . . . . . . 164

Pupil imaging on AO-DM . . . . . . . . . . . . . . . . . 166

12.3.2. Spectroscopic performance . . . . . . . . . . . . . 167

Ammonia ......................... 171

Methane isotopologues . . . . . . . . . . . . . . . . 172

12.3.3. Performance summary . . . . . . . . . . . . . . . . . 173 
In this chapter, I present an analysis of the performance of the NAGICS concept. While the general idea, scope, and design of the flexible NAGics system have been the focus of the previous chapter, I now develop a specific configuration (baseline design). This baseline design, described by a set of design parameters, is then modelled in detail and all relevant system properties computed. Further evaluation and performance discussion is then based on this reference design.

\subsection{Identifying a baseline design}

The primary design parameters of the NAGICS system are length $L$, optical bench separation $H$, and the allowed FoV $F$ (see Sec. 11.3.3 for definitions). All other (secondary) parameters will depend on the primaries and are determined iteratively for each configuration (choice of primary parameters), or are fixed by the requirements. Practical feasibility is of immediate concern for $L$ and $H$ (and to some extend to $F$, see below). That is, $L$ and $H$ are prone firstly to the mechanical constraints of integration and mounting feasibility. Note again that, given the specific CRIRES requirements, any increase of distances ultimately translates into an enlarged diverging beam diameter, which in turn must be accomodated. In other words, increasing $H$ or $L$ counteracts compactness and (mechanical) complexity. While $L$ scales nearly linearly (and over a large range) with the attainable OPL, complexity (and cost) does not. It is therefore mandatory to compromise for a controlled baseline solution. I discuss the choice of primary design parameters in the following.

\subsubsection{Opto-mechanical assembly height}

The NAGICS DL and RCRF sub-systems are mounted on an elevated optical bench on top of the CRIRES warm optics bench (AO-table; cf. Fig. 11.18). The elevation of the NAGICS bench $H$ is defined as the separation between the level of the optical axis (OPX) on the AO-table, and the level of the optical axis on the NAGics bench. $H$ must mechanically accomodate all units on the AO-table, allow for the bench structures, the second optical bench thickness, mirror mounts for appropriately sized mirrors, plus margin. At the same time, $H$ must be minimized to control the diverging beam diameter, and hence the succeeding optical apertures.

The OPX on the AO-table is $150 \mathrm{~mm}$ above the bench. The heighest unit on that bench is the calibration mirror bridge (feeding the calibration lamps if the fold mirror is hauled into place) with $350 \mathrm{~mm}$, ie. extending $200 \mathrm{~mm}$ atop the OPX. The derotator unit needs about $180 \mathrm{~mm}$ clearance around the OPX. Typical optical breadboard benches have $\sim 60$ to $110 \mathrm{~mm}$ strength $^{1}$, and mirror mounts may contribute with some $50 \mathrm{~mm}$ clearance. With an additional largest mirror-diameter of $100 \mathrm{~mm}$ and some $40 \mathrm{~mm}$ margin, I estimate the minimum required OPX separation to be $H=400 \mathrm{~mm}$ as baseline. This should leave enough space to access also the units on the calibration carriage slide, eg. the short-path gas cells.

The scaling behaviour of NAGICS is illustrated in Fig. 12.1. Here, the required minimum mirror aperture diameters $D_{i}$ are computed as a function of $H$, while keeping $L$ fixed. $D_{i}$ is evaluated for FM2, FM6, RCM1, and RCM2 (cf. Fig. 11.19) in terms of the size of the footprint caused by an on-axis field point. While the footprint on a particular surface may be offset for off-axis field points (but still be of the same size), and is thus strictly not exact

\footnotetext{
${ }^{1}$ eg. Newport precision grade optical tables
} 


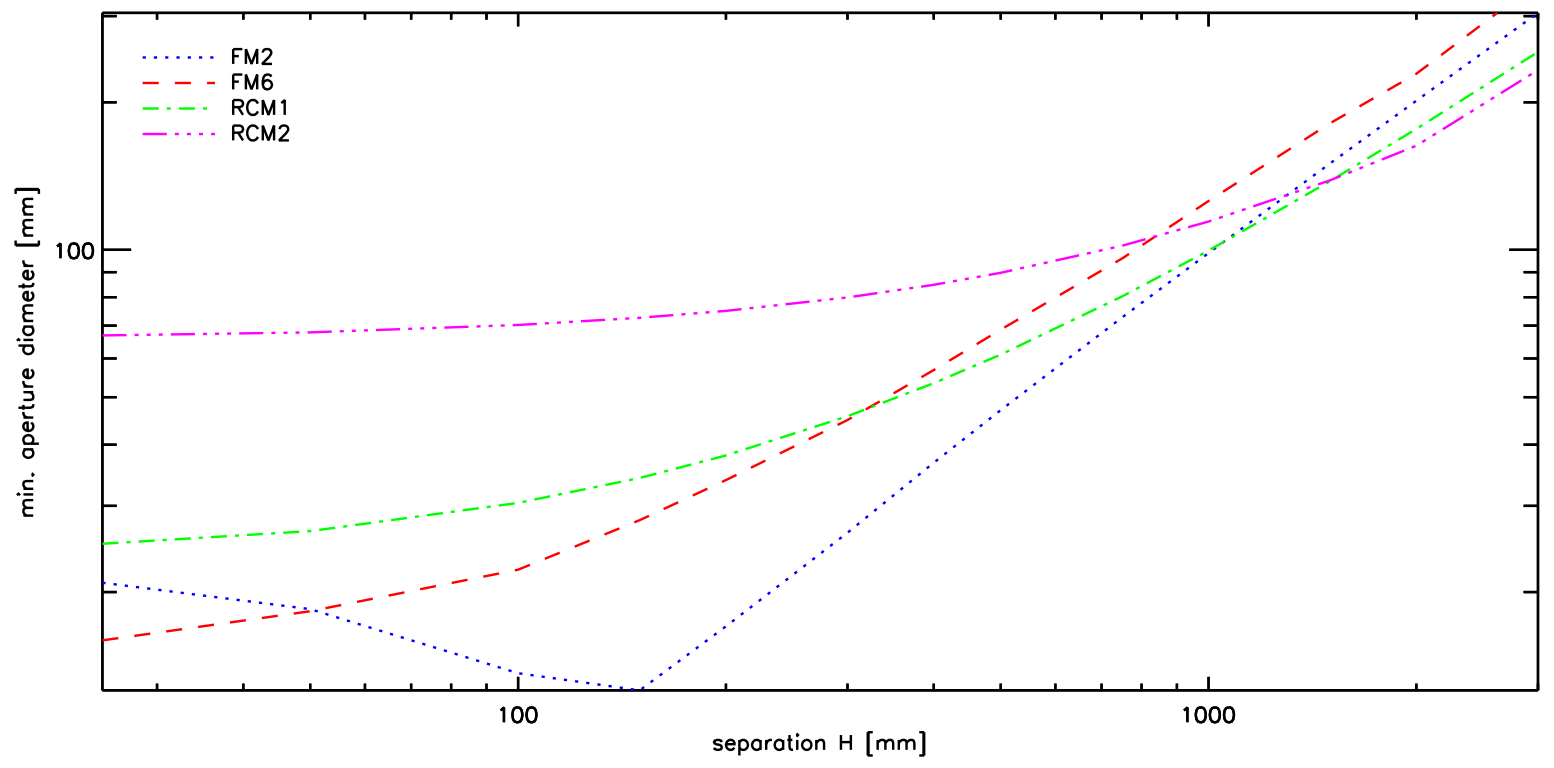

Figure 12.1.: Scaling behaviour of the baseline design with the optical bench separation parameter $H$. The required minimum mirror diameters are evaluated as a function of $H$, and plotted for the flat folding mirrors FM2 and FM6, and for the re-collimators RCM1 and RCM2. Note that the behaviour is different for each mirror, and does not scale linearly nor uniformly over a large range in $H$. For the given CRIREs setup, $H$ should be no smaller than 300 to $400 \mathrm{~mm}$. This translates into well controlled mirror diameters. For $H>700 \mathrm{~mm}$, several apertures start to quickly exceed $100 \mathrm{~mm}$ in diameter, and thus become rather unfeasible to MAIT. For $H<300$ the complexity becomes increasingly advantagous, but physically unfeasible for the CRIRES setup.

for a FoV > 0, Fig. 12.1 is nevertheless a good measure of the relative behaviour between the different mirrors. The $D_{i}$ scale differently, so that a feasibility range can be identified. For very small separations $(H<300 \mathrm{~mm})$, the system cannot be implemented for the current CRIRES setup (see above). On the contrary, for large $H>700 \mathrm{~mm}$, several mirrors exceed $100 \mathrm{~mm}$ diameter, and thus become increasingly complex (and mounting clearance will require different estimates than those above). A baseline of $H=400$ is thus in the optimum range to control the mirror dimensions.

\subsubsection{Optical bench dimensions}

The AO-table geometry restricts the attainable cell length $L$, as a straightforward mounting of the NAGics bench on top is desirable. Thus, it should not extend beyond the borders of the AO-table (to not cause Nasmyth platform obstructions, and to ease re-use of the current design of light covers). This will also allow for a simplified mounting of the second bench on structural posts erected on the $\mathrm{AO}$ bench, given load restrictions and vibration control is not a concern.

The AO-table dimensions are $1100 \times 1725 \mathrm{~mm}(\mathrm{~L} \times \mathrm{W})$, with the longer side perpendicular to the Nasmyth axis, and a cut-out of $100 \times 1050 \mathrm{~mm}$ to adapt to the round cryostat vessel (for details, see Figs. 11.5 and 11.6). The Nasmyth OPX, and thus the location of the FO sub-system, is not in the middle of the AO-table, but has a shorter clearance of $550 \mathrm{~mm}$ to 
the side. This means that, due to its inherent symmetry, the NAGICs RCRF half-length must be $L<550 \mathrm{~mm}$ (including mirror mounts and clearance for enclosure walls), in order to not protrude the AO-bench. A maximum $L=500 \mathrm{~mm}$ is deemed feasible with safety distance margins for the RCRF mirrors.

\subsubsection{Field of view}

The minimum apertures required are also a function of FoV. They scale linearly with the FoV, with the maximum gradient at any FP, and the least effect at any pupil plane. The FoV is thus mostly of concern for optical surfaces close to a focus. As an example, the first fold-out mirror (FM1) growths from $\sim 13$ to $\sim 66 \mathrm{~mm}$ in diameter for an on-axis and a $50^{\prime \prime} \times 50^{\prime \prime}$, respectively. While the full beam diameter in front of the FO sub-system is $54 \mathrm{~mm}$ (and thus barely fits the other calibration units on the carriage slide), the numbers are increased on the folding units due to the projection effect. With $H=400 \mathrm{~mm}$, the numbers are 50 and $97 \mathrm{~mm}$ diameters for FM6, respectively. In effect, for the full CRIRES-FoV the fold-mirrors (and the powered ones, too) become too large to easily mount with the constraints estimated for $H$. The FoV supported by NAGICS is thus to be reduced. Although CRIRES observations through NAGICS would, in principle, work with a minimal FoV around the on-axis, this would strongly limit the use of the AO. The center target object must then be the AO star, which is restricts AO usage to bright and blue enough stars. More importantly, a "missing field" would render target acquisition, placement of the star on the spectrograph's slit, and possibly guiding very inefficient. A minimum FoV of $5^{\prime \prime} \times 5^{\prime \prime}$ is deemed indispensible (cf. Sec. 11.2).

For selected fold-units, Fig. 12.2 plots their size depending on the FoV (for a configuration with $H=400 \mathrm{~mm}$ ). The largest unit is FM6, and its size is important to control as it also determines the necessary window size into the gas container vessel. To keep the windows reasonable small, a FoV of no more than $20^{\prime \prime} \times 20^{\prime \prime}$ seems advantagous.

Another important consequence the FoV has is on the DL sub-system. Due to its compact design with adjacent $45^{\circ}$ folds, an increase in FoV requires additional spacing between the FM3-FM6, as larger sizes would otherwise not fit, and cause vignetting. This additional spacing, as a consequence, is lacking towards the NAGICS Nasmyth FP: the more separation the DL sub-system takes, the closer FM8 approaches the FP. This is to be avoided as a) the FP should not sit too close to the mirror surface, and b) as some minimal mounting space is needed for FM8 on the carriage. In this way, an FoV larger than $15^{\prime \prime} \times 15^{\prime \prime}$ becomes unfeasible. As the CRIRES+ entrance slit will be limited to $10^{\prime \prime}$ (to suit the cross-dispersed echelle format, Follert et al. 2013), there is no strong reason to enable a FoV larger than that. ${ }^{2}$ Therefore, $10^{\prime \prime} \times 10^{\prime \prime}$ is baselined for NAGics.

The FoV is also a parameter that strongly influences the attainable optical quality, as aberrations become far more difficult to control the farther off-axis the FoV gets. Compensating for these off-axis effects will always be at the cost of the central field performance in a strongly decentered system like NAGICS. This aspect is further detailed when assessing the performance for a particular configuration in Sec. 12.3.

\subsubsection{Optical path length}

The achievable additional optical path length (AOPL) with NAGICS is the OPL inside the gas vessel. This is the distance light travels from the vessel entrance window to its exit window

\footnotetext{
${ }^{2}$ A larger FoV than the slit-length is nevertheless helpful for the slit-viewer, for target acquisition, and AO-guiding.
} 


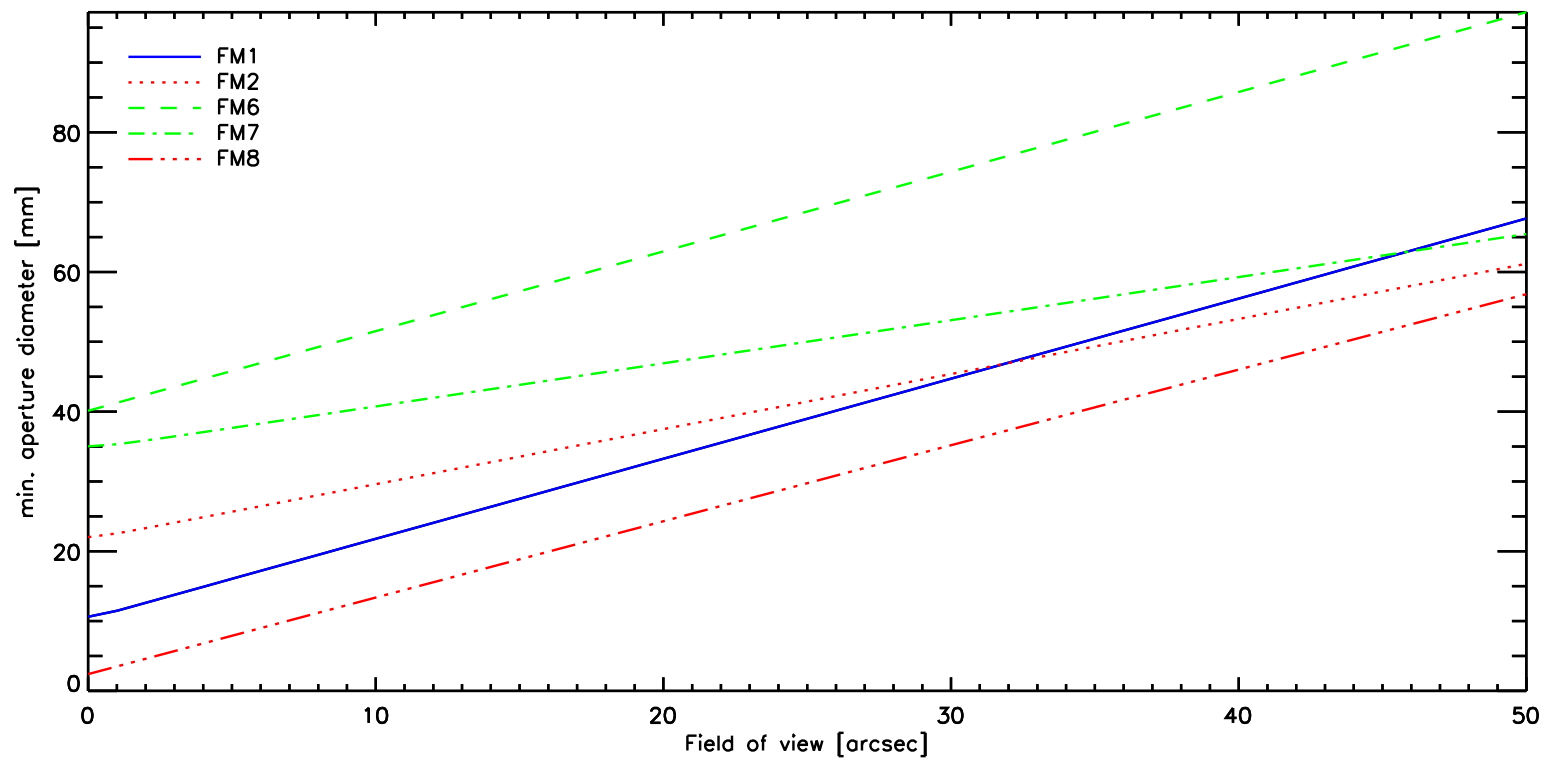

Figure 12.2.: Folding mirror apertures as a function of allowed FoV. For placement of the FM, see Fig. 11.19. Diameters are given for squared FoVs, $50^{\prime \prime} \times 50^{\prime \prime}$ is the full CRIREs field.
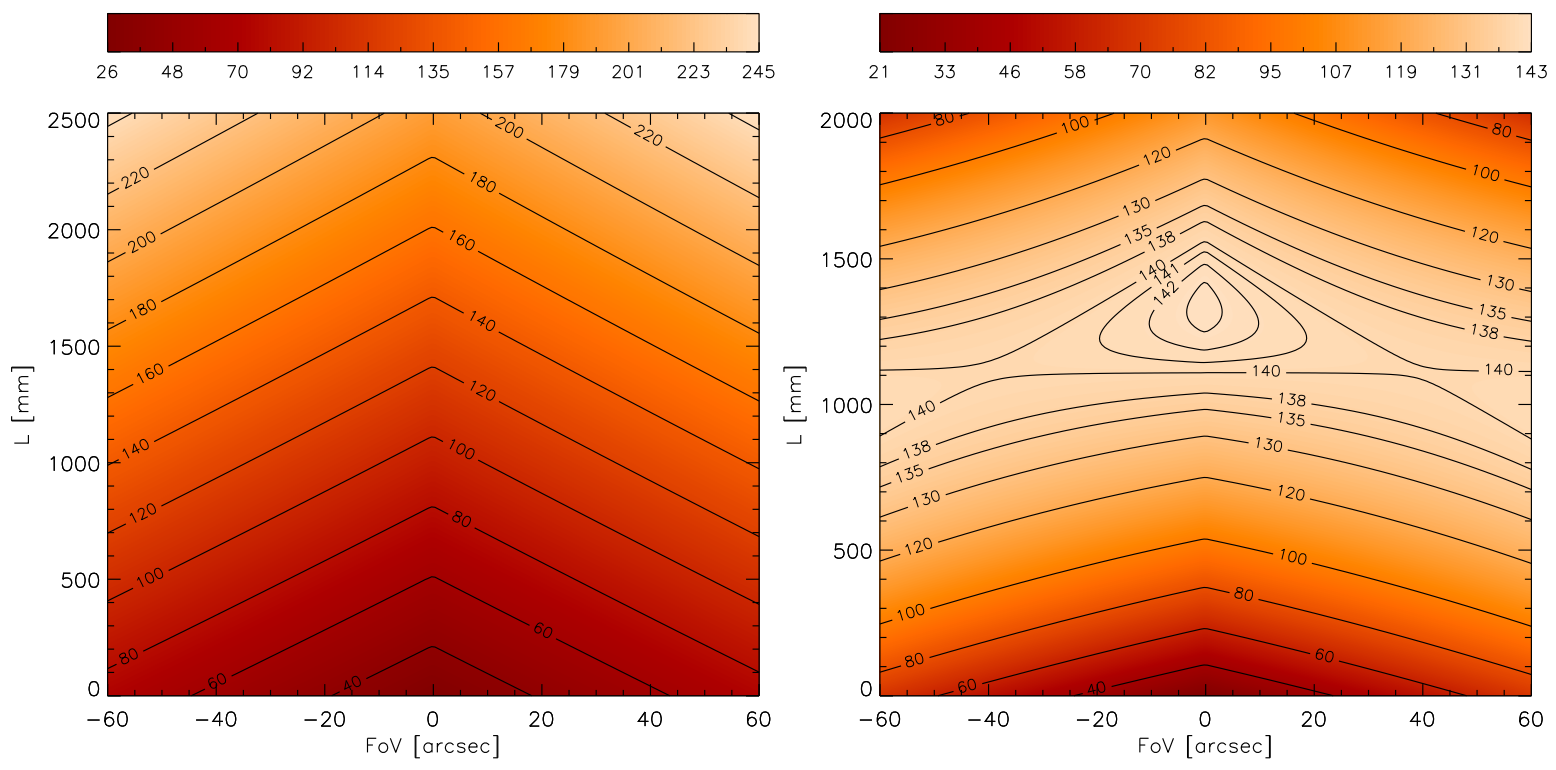

Figure 12.3.: Scaling behaviour of the baseline design (with the optical bench separation parameter $H=400 \mathrm{~mm}$ ). The contour maps give the required minimum reflective aperture (in $\mathrm{mm}$ ) of the RCM1 mirror unit (left panel) and of the RCM2 mirror unit (right panel) in relation to the targeted FoV and the cell half-length $L$.

(in the case the container encloses the entire RCRF; otherwise a constant length must be deducted). The usable AOPL is thus simply tied to the half-length $L$, and scales roughly like $\times L$. The exact AOPL depends also on the arm separation $S$. This means that for the smallest practical NAGICS configuration with about $200 \mathrm{~mm}$ half-length, the AOPL gets around $1.6 \mathrm{~m}$. 
This is almost an entire order of magnitude longer than the current CRIRES calibration cells, and a factor of 3.2 larger than the longest conventional cell under construction (D'Amato et al. 2008).

The influence of the OPL (thus $L$ ) and the FoV on the required minimum apertures for the RCRF units is calculated and displayed in Fig. 12.3. The contour maps demonstrate, that while the first RCRF unit (RCM1) depends linearly on $L$ and FoV, this is not the case for the RCM2 unit.

\section{Gas vessel dimensions}

The geometry of the vessel container follows closely the targeted cell half-length $L$ and $S$. The resulting vessel length thus scales with $2 L$, and with $S$ in width, plus some overhead and clearance depending on the required mirror apertures. A detailed construction design for the container is outside the scope of this work. I baseline the general vessel concept outlined in Sec. 11.3.5, §1. The principle is envisioned to encompass a steel container with lid and metallic vacuum seals, that hosts an optical bench inside. The RCRF sub-system is independently mounted on that bench, while the two windows (entrance and exit) are attached to the container. The reasoning against a nested vessel is simply the anticipated loss of light due to the multiple $(10 \times)$ window passages (see Sec. 12.3.1). Such a gas cell is foreseen to allow for fuelling with a suitable gas to be selected, and it should also be feasible to characterize it by means of an external FTS.

\subsubsection{Mirror properties}

\section{Powered mirrors}

The type of curvature of a mirror is highly correlated to manufacturing complexity and cost. Spherical shapes are straightforward to MAIT, but suffer from substantial (spherical) aberrations. This is particularly harmful to the NAGICS concept, as it relies on heavy off-axis operation of the RCRF mirrors. Parabolic shapes are much more versatile in the NAGICS context, yet to aim for the best attainable optical performance, I baseline the RCRF surfaces as free conical shapes, ie. any conical constant that fits best (see also Sec. 13.1.2 for restrictions).

\section{Folding mirrors}

No special requirements apply to the folding mirrors. All units can be of the same $45^{\circ}$ kind and build, dictated by FM6 $(\varnothing 60 \mathrm{~mm})$.

\section{Coatings}

The NAGICS design adds 12 reflecting surfaces to the CRIRES optical train before light enters the spectrograph. The least possible number of mirrors must be aimed for in the system, and high-reflectance $\varrho$ of all surfaces must be ensured in order to keep the instrument efficiency as high as possible. A standard high-reflectance aluminum coating $(\varrho>95 \%)$ introduces losses of nearly $50 \%$ in the NIR. Silver or gold HR-coatings ( $\varrho \gtrsim 98 \%$ in the NIR) are therefore baselined. Their efficiency will be analyzed in Sec. 12.3.1. 


\subsubsection{Baseline design parameters}

The basic design parameters for the NAGICS baseline configuration are summarized in Table 12.1. This configuration is modelled in detail and iterated until converging at a stable solution for all involved elements and requirements. Its anticipated performance can then be evaluated and compared to the nominal CRIRES model.

\subsection{Model performance verification}

A benchmark analysis of the NAGICS baseline design solution (also) relies on a comparison with a reference setup. For NAGics, the reference is an optical model in which the wavefronts enter the telescope, and are propagated until arriving at the spectrograph's entrance slit. Thus, this involves the VLT and the CRIRES warm optics as presented in Sec. 11.2.5ff. Essentially, this model is identical to the NAGICS model, except for the actual NAGICS device (eg. inactive, or taken out). In this section, I first verify the modelling of the VLT, and then the combined VLT and CRIRES, before assessing some key performance metrics for the latter.

\subsubsection{VLT model at Nasmyth}

The model of the VLT in Nasmyth configuration according to the specifications in Sec. 11.2.5 is set-up in a Zemax environment. I verify the model behaviour by propagating large numbers of incident rays to the FP, and analyze their distribution and properties. The rays are usually defined by their wavelength and originating coordinates, as well as the coordinates of pupil interception.

The VLT-model works as anticipated and correctly reproduces the expected behaviour. Note that the model cannot consider environmental effects such as atmospheric seeing conditions, and neglects manufacturing deficiencies and dynamic effects of operation (noise, shake, guiding errors, etc). The model thus gives a description of the physical behaviour of the optics for the ideal case of mechanically perfect mirrors. Although the image quality of a large

Table 12.1.: Summary of NAGICS baseline design parameters

\begin{tabular}{lccr}
\hline \hline Parameter & Symbol & Limitations & Baseline \\
\hline Cell half-length & $L$ & $\leq 500 \mathrm{~mm}^{a}$ & $400 \mathrm{~mm}$ \\
Bench separation & $H$ & $>300 \mathrm{~mm}^{b}$ & $400 \mathrm{~mm}$ \\
Add. opt. path length & AOPL & $>1.6 \mathrm{~m}$ & $\sim 2.96 \mathrm{~m}$ \\
(unvignetted) FOV & FoV & $\leq 15^{\prime \prime} \times 15^{\prime \prime}$ & $10^{\prime \prime} \times 10^{\prime \prime}$ \\
Arm separation & $S$ & $\geq 160 \mathrm{~mm}^{c}$ & $180 \mathrm{~mm}$ \\
Gas vessel type & & transmission losses & enclosure, two windows \\
Mirror coatings & & protected Al, Ag or Ag & (see Sec. 12.3 .1 ) \\
Mirror curvatures & & - & $-4250 \mathrm{~mm}$ to $+4200 \mathrm{~mm}^{d}$ \\
Mirror conics & & - & -2.7 to $+1.6^{d}$ \\
\hline \hline
\end{tabular}

Notes.

(a) Imposed by AO-bench geometry.

(b) Limited by existing CRIRES warm optic units.

(c) Depends on $L, H$, and FoV.

(d) Exakt values subject to choice of window substrate. 

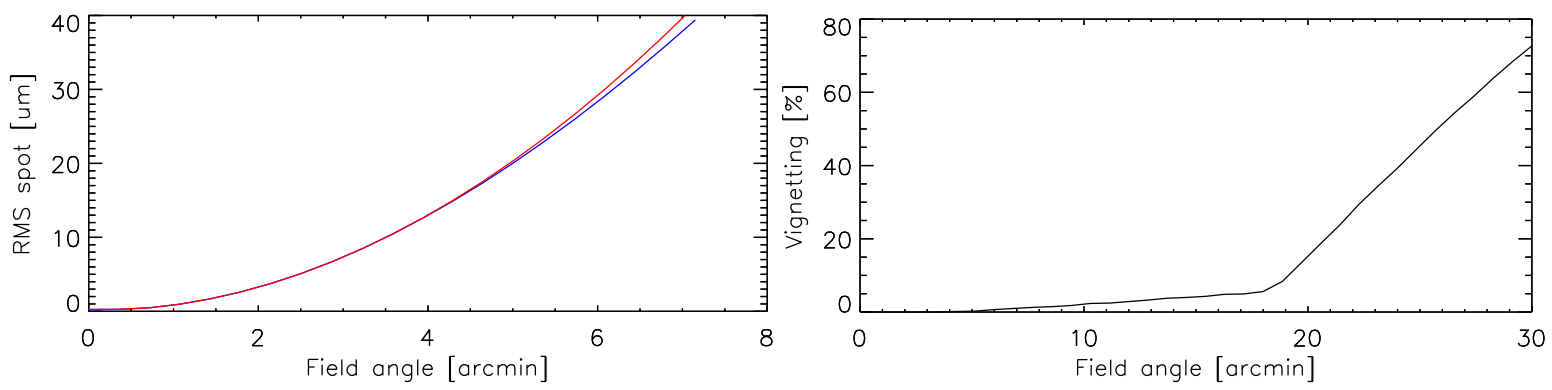

Figure 12.4.: Model performance at the Nasmyth FP, measured as spot radii (RMS deviation from centroid position) over the FoV (left panel). The distribution in $\mathrm{x}$ (red) and y (blue) is computed separately. Note that at larger off-axis angles astigmatism becomes apparent. The right panel plots vignetting as a function of FoV. At $10^{\prime}$, about $2.2 \%$ of the field are vignetted.

telescope cannot be quantified entirely by simple metrics such as resulting image spot size or PSF (Dierickx 1992), in our idealistic case such analysis is very helpful to assess the design performance (operational VLT optical performance tests can be found elsewhere, eg. Guisard et al. 2000). Fig. 12.4 (left panel) shows the resulting spot size (RMS) of a point source, traced by a finite number of rays through the model, as a function of the field angle on the sky (ie. projected angular distance from the telescope optical axis). The spot size is measured on the curved FP (field curvature of the Nasmyth FP is not a concern over the CRIRES FoV, and can be neglected). At $2.3 \mu \mathrm{m}$, the diffraction limit is about $42 \mu \mathrm{m}$, so the telescope model already correctly delivers diffraction limited performance over the entire unvignetted FoV (7'15). Note that the actual seeing thus will always limit the real performance, as is desired. The right panel shows the fraction of vignetting over the telescope's FoV. These numbers are in excellent agreement with Dierickx et al. (1995), and indicate that the exact geometry of the optical elements are correct in the model.

The resulting PSF is shown in Fig. 12.5 as the fraction of encircled energy, measured from the centroid position on the FP. The left panel gives the distribution for three extreme field angles for a wavelength of $2.3 \mu \mathrm{m}$. The on-axis performance is limited by its diffraction pattern. The seeing limitation under the best attainable conditions (seeing $\sim 0$ ". 4 ) holds up to almost the entire $30^{\prime}$ FoV. Energy concentrations for finite size objects are computed in the right panel of Fig. 12.5, and are indicative for the spatial (pixel) sampling required in the FP.

\subsubsection{CRIRES model}

The normal CRIRES optical train (ie. without insertion of a calibration cell) until after the AO module is entirely comprised of reflective elements. This guarantees a high performance over the entire CRIRES-range of wavelengths $(0.95-5.3 \mu \mathrm{m})$, which would physically not be possible with refractive elements. There is, however, one refractive surface that cannot be circumvented: the entrance window to the cryostat. The window has great impact on the overall optical performance, and deserves a separate treatment in the analysis. By looking at the performance achieved with and without the entrance window, one can better understand the effect this bottleneck has on the system. Then, in a second step, the deteriorating effect of the window can be addressed when finding an optimal solution for the NAGICS baseline design. 

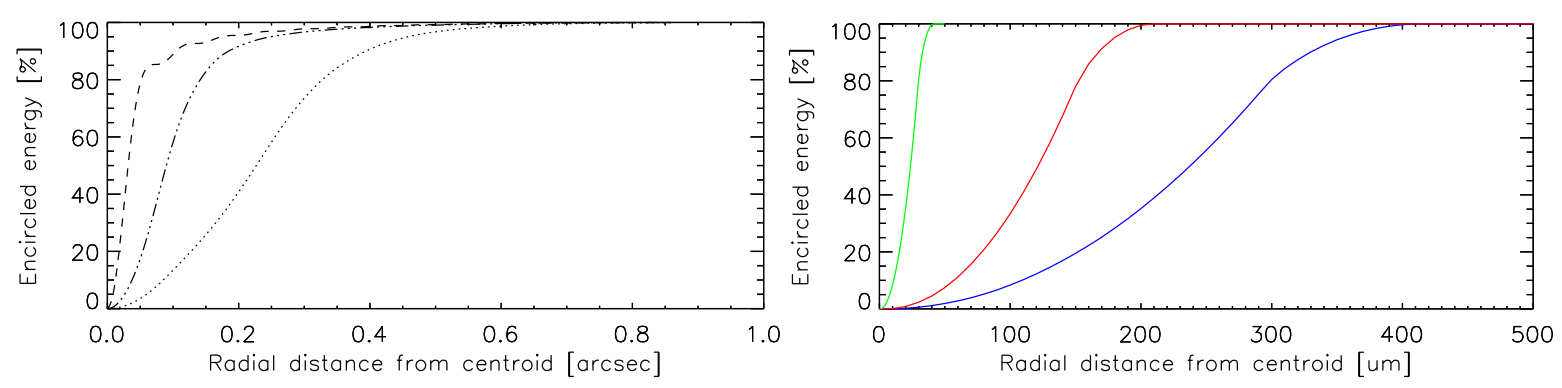

Figure 12.5.: Analysis of the image quality at the VLT-Nasmyth focus. Plotted is the fraction of encircled energy as a function of the PSF radius for an ideal point source (left panel), and sources with finite size (right panel). The telescope model neglects actual manufacturing tolerances and assumes ideal alignment and no seeing effects, and so gives the theoretical optical capabilities.

Left: Point sources with $\lambda=2.3 \mu \mathrm{m}$ at zero field angle (on-axis), $7^{\prime}$, and $30^{\prime}$ are propagated through the model, corresponding to the dashed, dashed-dooted, and dotted curves, respectively. The VLT optics are purely diffraction limited, with $80 \%$ encircled energy within a $0.10 \mathrm{PSF}$ in the on-axis case (note that the Airy-disc radius is visible as the first dip at $0 . " 072$ ).

Right: Extended homogenous disc objects of $0 . \prime 1,0 . " 5$, and $1{ }^{\prime \prime} 0$ are simulated with the VLT model. The images at the Nasmyth FP yield the green, red, and blue curves of encircled energy fractions, respectively. Note that neither active nor adaptive optics are considered here.

\section{Field dependent Strehl ratio}

CRIRES is designed for a full field of $50^{\prime \prime} \times 50^{\prime \prime}$. I quantify the optical performance of the warm-optics by first looking at the focal plane after the AO-module, in order to verify the model against the Delabre et al. (2000) specifications.

The Strehl ratio gives a useful metric to describe the system, it is defined as the ratio of the peak intensity of a system's diffraction PSF over the peak intensity of the diffraction PSF in the absence of aberrations. Thus, the peak intensity of a point source of given wavelength, located anywhere in the FoV, and suffering from diffraction, aberrations, and other deteriorating effects on the PSF, is devided by the peak intensity of the on-axis point source, suffering purely by diffraction.

The Strehl ratios obtained at the FP $230.1 \mathrm{~mm}$ behind the AOM3 (thus ignoring for a moment the dichroic entrance window) are highly determined by the effects of the spherical AO mirrors. Fig. 12.6 and Fig. 12.7 represent the corresponding Strehl ratios at the VLT Nasmyth FP and at the AO-focus, respectively. It is clear that the minimal aberrations of the VLT only become noticable at large field angles (note the different ranges in the figures), while for CRIREs the AO introduces significant losses within the $50^{\prime \prime} \times 50^{\prime \prime}$ FoV. Note that the Strehls given here are computed without wavefront correction by an AO in closed-loop. The purpose of the AO is, naturally, to improve the actual PSF (spatially and in time), in order to increase the observable Strehl. Here, however, I am interested in asessing the relative performance of CRIRES without and with the NAGICS device. Hence, the active AO capabilities are not needed here (and strongly depend on the instantanous conditions anyway).

As the system is slightly decentered due to the tilted operation of the AO mirrors, I plot 


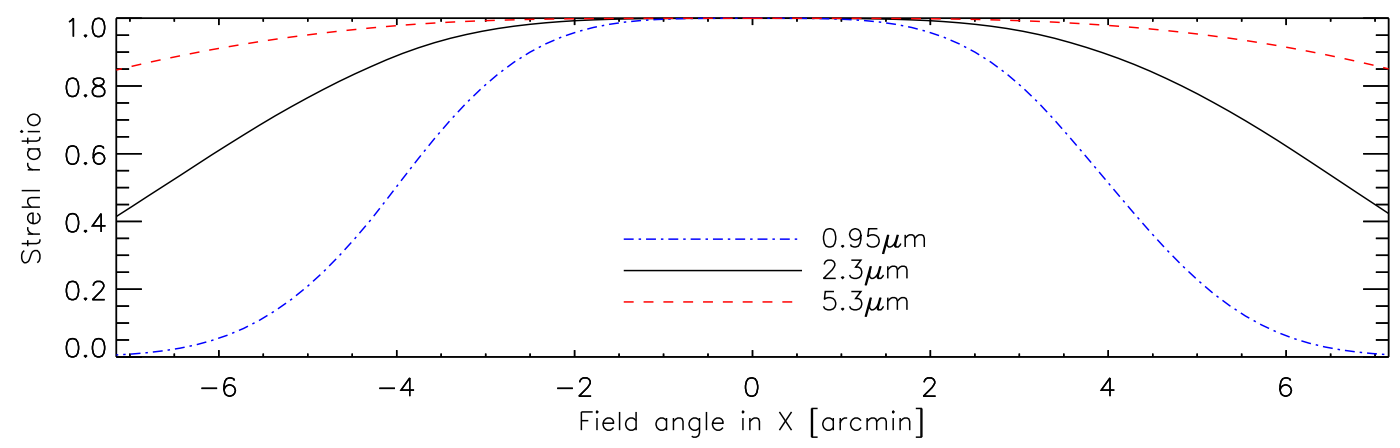

Figure 12.6.: Strehl ratios $s$ at the Nasmyth focal plane, for three wavelengths, as a function of horizontal field angle (identical to the vertical profile due to axi-symmetry. The VLT design provides diffraction limited $(s \geq 0.8)$ imaging up to about $3^{\prime}$ near $1 \mu \mathrm{m}$, and across the full unvignetted $\sim 7^{\prime}$ longward of $5 \mu \mathrm{m}$.

the Strehl separately for the horizontal and vertical field angles in Fig. 12.7 (notice the asymmetry in $y$ ). The impact of the warm-optics is negligible very close around the OPX $\left( \pm 1^{\prime \prime}\right)$, but becomes increasingly larger at higher field angles. The system can maintain diffraction limited performance (commonly referred to for $s \geq 0.8$ ) over the full field at the longest operating wavelengths, while this reduces to about $4.5^{\prime \prime} \times 4.5^{\prime \prime}$ approaching the bluer limit at $0.95 \mu \mathrm{m}$ (recall that the shorter wavelengths are reflected for WFS). Two reasons need to be mentioned: First, the overall degradation is due to the AO-module, which employs spherical mirrors, thus resulting in significant astigmatism and field curvature as mentioned previously (the spherical aberrations are mostly compensated for). Second, the higher Strehl ratios at longer wavelengths are a result of the diffraction limit, which scales with $\lambda / D$. This means the peak intensity of the purely diffraction limited point source (no aberrations) decreases, so $s$ effectively gets larger with $\lambda$.

\section{Impact of the dichroic entrance window on Strehl}

The situation is significantly worsened when the Strehl ratios are measured at the instrument focal plane, ie. the location of the CRIRES+ slit after light passes the cryostat entrance window (Fig. 12.8). The best performance is now achieved not on-axis, but $+1^{\prime \prime}$ in vertical direction (noticable for visible/NIR wavelengths). This is caused by the plane-parallel plate window, and reduces the overall efficiency of the instrument by about $10 \%$ when observing around $1 \mu \mathrm{m}$.

\section{Chromatic focal shift}

It is important to realize two consequences that the entrance window has on the focal plane. This is first a wavelength dependent focal length, resulting from the material's dispersion properties (axial color defect). As for any glass, $\mathrm{CaF}_{2}$ has a wavelength dependent refractive index, and so the large wavelength range involved here suffers from quite different OPDs and incident angles when traversing the glass window (albeit the window has no curvature).

The chromatic focal shift along the optical axis (see also Fig. 12.22) amounts to a maximum offset of $2.65 \mathrm{~mm}$ between 0.95 and $5.3 \mu \mathrm{m}$, which must be compensated for by refocusing for a given wavelength. Otherwise, this defocus translates into a loss of efficiency: At $0.95 \mu \mathrm{m}$, the extreme $\Delta f=2.65 \mathrm{~mm}$ results in a loss of Strehl of $\sim 100 \%$ already on-axis. At $5.3 \mu \mathrm{m}$, 


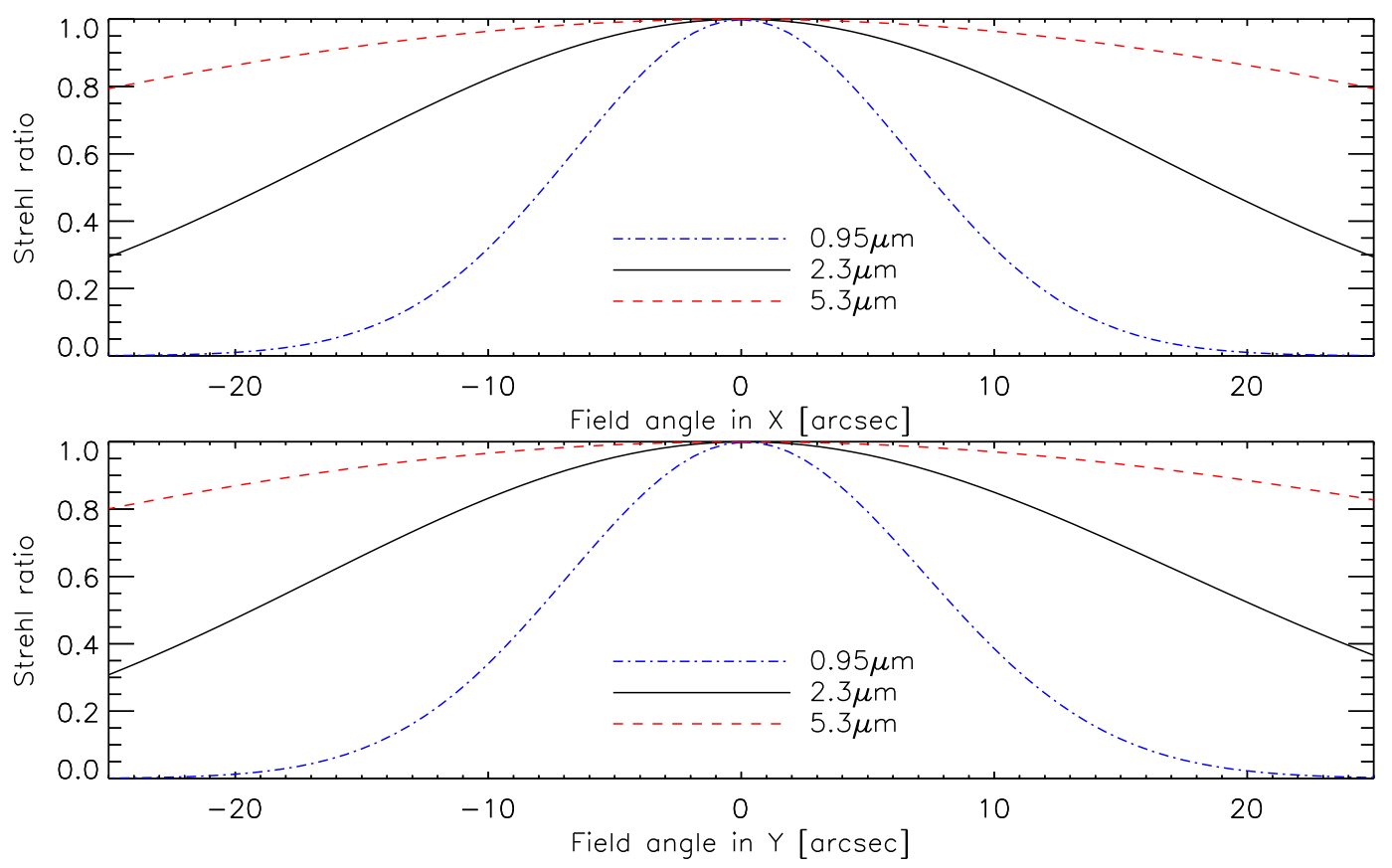

Figure 12.7.: Strehl ratios $s$ for the limiting wavelengths (plus central $K$ band) of CRIRES at the focal plane of the AO-module, plotted for the horizontal (top panel) and vertical field angle (bottom panel). While the full FoV provides diffraction limited $(s \geq 0.8)$ performance at $5.3 \mu \mathrm{m}$, this range shrinks to about $\pm 5^{\prime \prime}$ around the optical axis at the blue edge of the NIR.

the effect is more subtle, and yields a loss of $20 \%$ Strehl on-axis, and is worse off-axis; in any case, the performance is highly degraded. In the case of $2.65 \mathrm{~mm}$ uncorrected focus offset, the system is no longer diffraction limited at any wavelength nor any field angle. For smaller defocus amounts, the degradation is correspondingly less. Note that practically only the vertical performance is of importance for the spectrograph, as this is the orientation of the slit. I plot the Strehl ratio as a function of defocus for three wavelengths in Fig. 12.11, each for three angles along the vertical field (ie. min, center, and max along slit orientation). The zero-point is given by the average focus position over the entire wavelength range, and over the entire FoV. The slit is oriented such that it is perpendicular to the window tilt, so the chromatic focal shift is symmetric about the slit center. I will revisit the defocus effect when the NAGICs is under study in Sec. 12.3.1.

The second effect owing to the tilted window is a wavelength dependent displacement in the FP (lateral color defect). An image of eg. a point source in the FP is shifted in the direction of the window tilt, depending on the wavelength. For the slit (in the FP), this means the image (target) needs to be repositioned for each wavelength, in order to be located on the slit itself. ${ }^{3}$ This effect is computed for the center wavelengths of the $Z$ to $N$ bands in Fig. 12.9, along the field angle in the direction of displacement. Fig. 12.10 is the corresponding representation in the plane of the slit.

Although the chromatic effects of the entrance window cannot be altered by NAGICS,

\footnotetext{
3 This is particularly important, as the slit is almost never viewed in the wavelength of the spectroscopic observation. Hence, if not corrected, the PSF of the corresponding wavelength does not fall into the slit (or in other words, the resulting Strehl ratio is strongly hampered).
} 

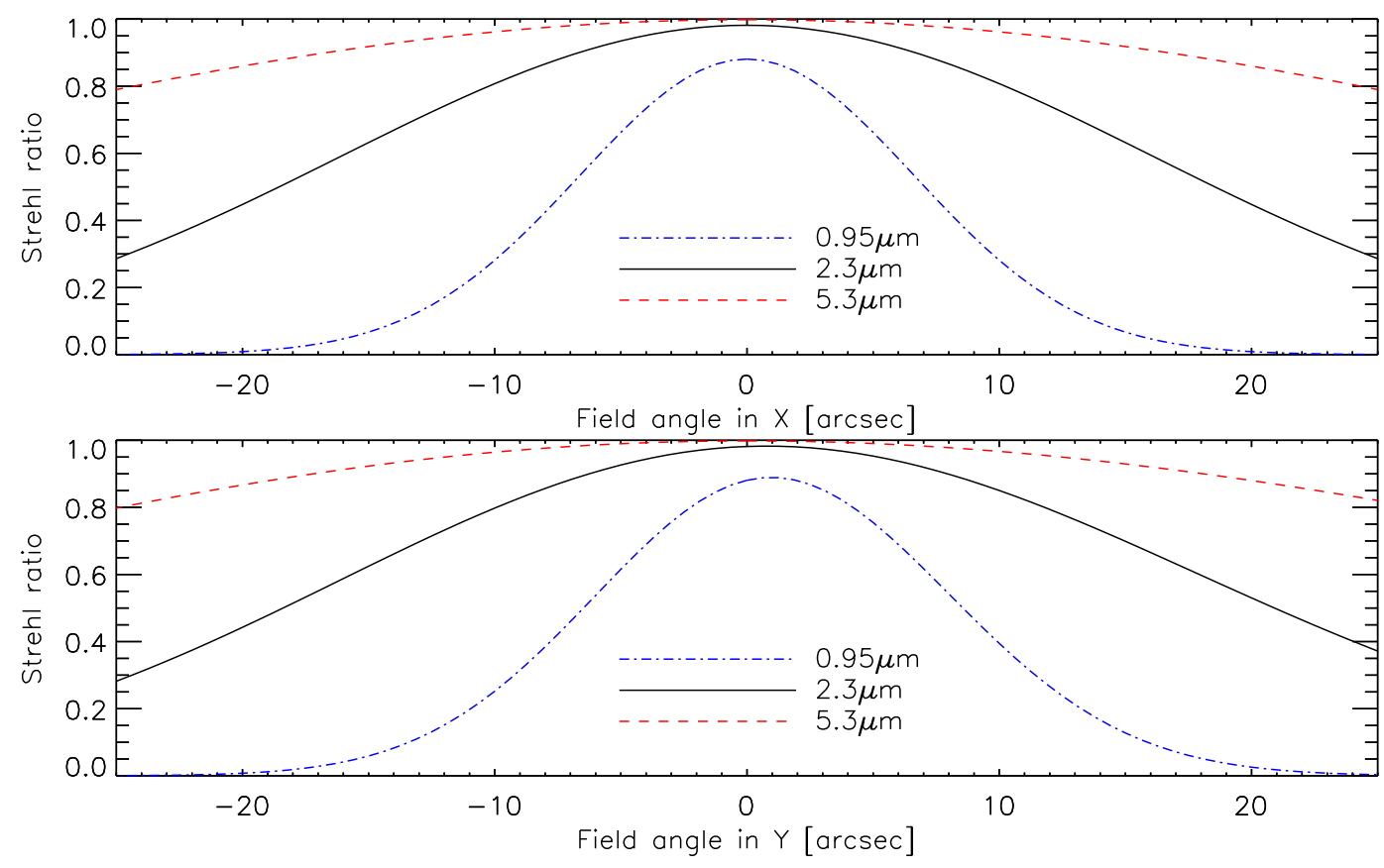

Figure 12.8.: Strehl ratios $s$ for the limiting wavelengths (plus central $K$ band) of CRIRES at the instrument focal plane on the entrance slit, after the dichroic entrance window to the cryostat. The horizontal (top panel) and vertical (bottom panel) field angle distribution differ slightly due to the tilted AO mirrors, and the tilted window. The impact of the $\mathrm{CaF}_{2}$ glass is a $10 \%$ loss at $0.95 \mu \mathrm{m}$, and negligible at the longest wavelength.

it is crucial to characterize them (they do not seem to be quantitatively documented ${ }^{4}$ ). This may serve as important input for the CRIRES+ operations (recall that CRIRES+ will have the entrance slit at the instrument focus after the window, while the current CRIRES accomodates relay optics at that location, and the entrance slit is situated only after that). The chromatism, as shown above, is a significant performance killer, yet the fundamental effect can only circumvented with a window design that is not or less tilted (which makes it incompatible with the AO design). Also, there is little choice for an alternative window material, as $\mathrm{CaF}_{2}$ offers the best availabe transmission over the desired, large wavelength range.

\section{Encircled energy fractions}

Energy concentrations on the slit are an important measure to quantify the throughput of a spectroscope. For a narrow entrance slit, and thus a high resolving power, the throughput is hampered by a target's flux that is lost at the slit, and so does not enter the dispersing elements. This is because the PSF on the slit is broadened by the transfer function of the optics involved, and by the dominating atmospheric seeing contribution. Adaptive optics through wavefront correction greatly helps to boost the PSF, ie. to increase the energy fraction passing through the slit.

\footnotetext{
${ }^{4}$ Delabre et al. (2000) does not seem to include chromatic effects into their analysis, they only show an
} uncommented figure of the chromatic displacement in the FP. 


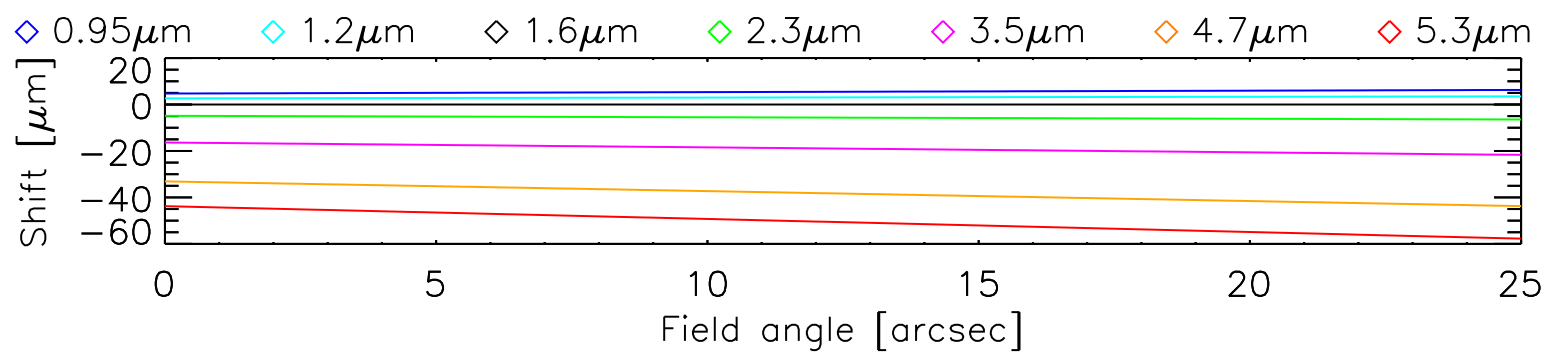

Figure 12.9.: Wavelength dependent lateral shift at the CRIRES+ slit, caused by the tilted $\mathrm{CaF}_{2}$ entrance window. The displacement is given in the direction perpendicular to the slit (tilt plane of the window), for the corresponding off-axis angles. The reference is given by the $1.6 \mu \mathrm{m}$ image.

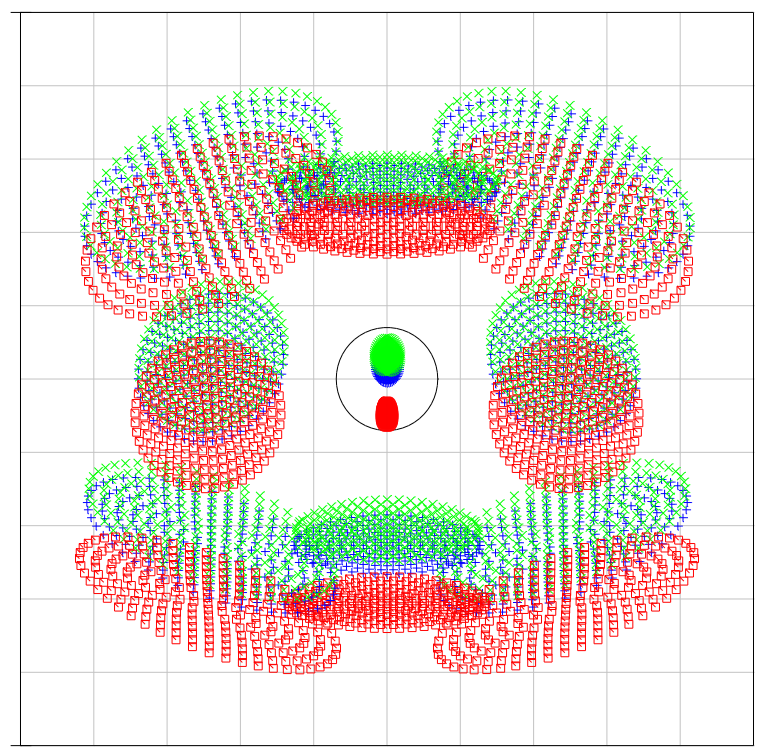

Figure 12.10.: Lateral wavelength dependent displacement at the instrument FP (at the CRIRES+ slit). The spot distributions sample the $50^{\prime \prime} \times 50^{\prime \prime}$ FoV (the FP shown covers $60 \mathrm{~mm}$ box length). The size of the spot distributions is exaggerated by $\times 100$ and given by the $42 \mu \mathrm{m}$ radius of the Airy disk (circle, for $2.3 \mu \mathrm{m}$ ). The entrance slit is in the horizontal direction. Colours represent $\lambda=0.95,2.3$, and $5.4 \mu \mathrm{m}$ (green, blue, and red, respectively). 

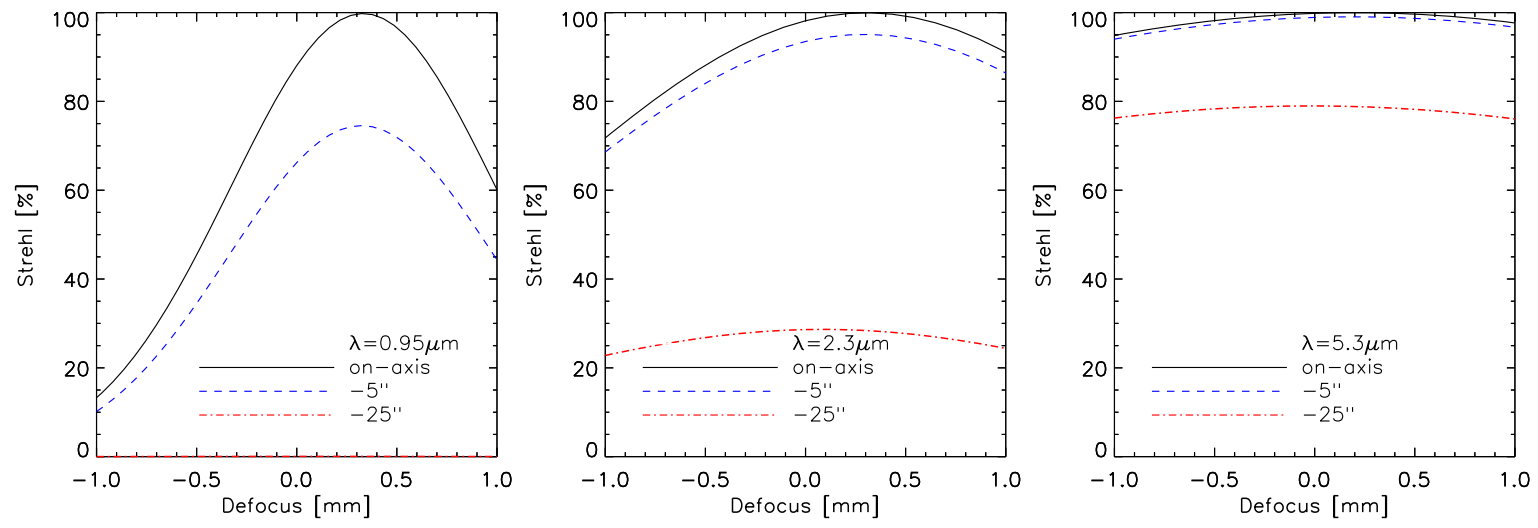

Figure 12.11.: Strehl ratios as a function of the defocus (bottom panels), resulting from a wavelength dependent focal shift in axial direction. Plotted are wavelengths of $0.95 \mu \mathrm{m}, 2.3 \mu \mathrm{m}$, and $5.3 \mu \mathrm{m}$ (left to right), for three field angles along the direction of the CRIRES+ slit (on-axis, $5^{\prime \prime}, 25^{\prime \prime}$ ).

The first broadening agent is determined by the physical properties of the optics, and may serve as a valuable benchmark for assessing the capabilities of the instrument. The CRIRES optics are designed to deliver an encircled energy concentration for a point source of $80 \%$ within $0.05^{\prime \prime}$ around the on-axis position, and up to $0.17^{\prime \prime}$ around the diagonal field edge at $\pm 25^{\prime \prime}$ (Delabre et al. 2000) ${ }^{5}$. My model of the CRIRES + optical chain reproduces these figures very well. Note that in my case the slit is located in the instrument FP, and there is no more pre-slit relay optics, which explains my slightly better resaults. With the suppression of the relay, the aberrations introduced by the $\mathrm{AO}$ module cannot be compensated anymore, and the impact at the slit needs to be considered. The encircled energy distributions my model delivers are shown in Fig. 12.12 for the boundary and central wavelengths $0.95 \mu \mathrm{m}, 2.3 \mu \mathrm{m}$, and $5.3 \mu \mathrm{m}$, probed at nine positions in the $50^{\prime \prime} \times 50^{\prime \prime} \mathrm{FOV}$ (on-axis, corners, and edges). These results set the stage against which NAGICS is to be compared.

\section{Spot sizes in the image plane}

To prepare a direct comparison of the image plane on the entrance slit for a) CRIRES without, and b) with NAGICs installed in the optical path, it is also useful to evaluate the image spot size of a point source across the field. Fig. 12.13 plots the RMS spot sizes for three wavelengths across a reduced $10^{\prime \prime} \times 10^{\prime \prime}$ field of view, ie. the field covered by the cell's baseline design. The Airy disk sizes are shown for comparison (circles), at the corresponding wavelengths. Each panel represents a field point at $(x, y)=(0 \pm 5,0 \pm 5)^{\prime \prime}$, with coordinates at the image plane given in $\mathrm{mm}$. Note that the FP is shifted $\Delta y=-3.65 \mathrm{~mm}$ at $2.3 \mu \mathrm{m}$, due to the entrance window. This shift is wavelength dependent, for the reasons discussed in the previous paragraphs (focal shift).

The nominal image quality is diffraction limited over the entire field, at all wavelengths. Even at the lower bound of $0.95 \mu \mathrm{m}$, the RMS spot sizedelivered by the model is below the Airy disk size $(r=17.4 \mu m)$. The RMS radius at the weakest points $(x, y)=(\mp 5,-5)^{\prime \prime}$ is

\footnotetext{
${ }^{5}$ As it is not specified, it is not clear from their Table 4.1 for which wavelength the calculations were performed, or if the numbers given are polychromatic. My results perfectly match the Delabre et al. data for a central wavelength of $\sim 2.3 \mu \mathrm{m}$, so I assume the calculations in their Table 4.1 refers to that monochromatic wavelength.
} 

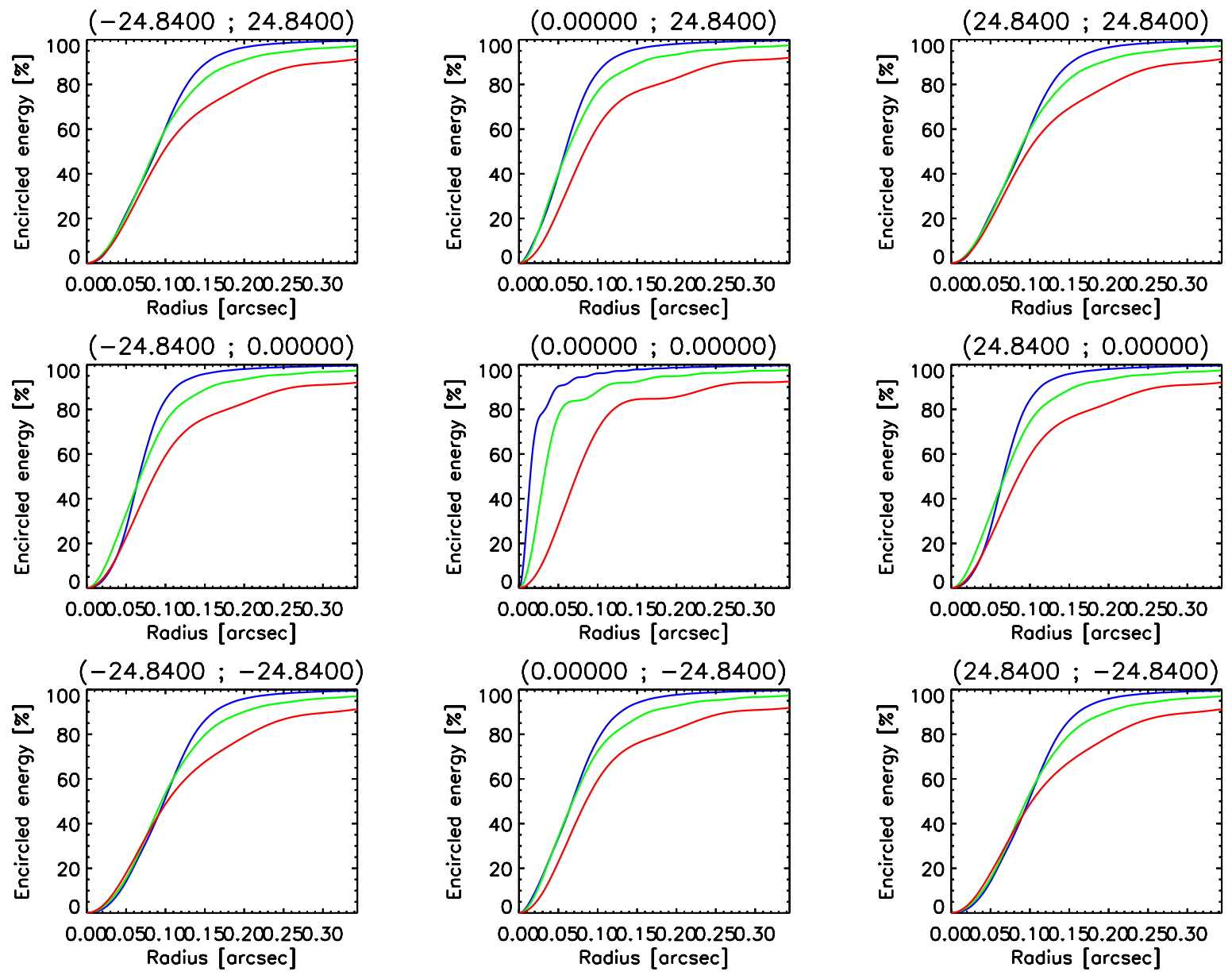

Figure 12.12.: Encircled energy fractions at the slit without NAGICS in place. Shown are the energy distributions centered at on-axis (middle panel) and the maximum field points in the $50^{\prime \prime} \times 50^{\prime \prime} \mathrm{FoV}$ (coordinates $x, y$ and radii in arcsec on the sky). The energy fractions are computed for the wavelengths $0.95 \mu \mathrm{m}, 2.3 \mu \mathrm{m}$, and $5.3 \mu \mathrm{m}$ (blue, green, red, respectively). The slit orientation is along the middle row (best performance). For a slit width of $0.2^{\prime \prime}\left(R=10^{5}\right)$, an ideal target at the slit center passes $\sim 100 \%$ and $85 \%$ of the energy into the spectrograph at $0.95 \mu \mathrm{m}$ and $5.3 \mu \mathrm{m}$, respectively (no atmospheric seeing assumed).

only $17.5 \mu \mathrm{m}$, thus still qualifies as "diffraction limited".

\section{Pupil imaging on AO-DM}

A pupil image on the AO-DM is achieved by the selection of curvatures and distances within the AO-relay optics. The pupil is needed to match the areas of probing by the WFS to the corresponding actuators of the deformable mirror, ie. the pupil image. The correspondence of pupil and its image on the AO-DM is plotted in Fig. 12.14 for both $x$ and $y$ coordinates in the pupil plane, with the corresponding coordinates on the AO-DM. The nominal diameter is $60 \mathrm{~mm}$. Deviations (lower panels in Fig. 12.14) are up to about $1 \%$ of the diameter in $\mathrm{x}$-direction. This corresponds to a small negative magnification. Along the $\mathrm{y}$-axis, the displacement is marginal ( $<0.5 \%$ of the diameter). The pupil image is flipped in $\mathrm{x}$ and $\mathrm{y}$ 


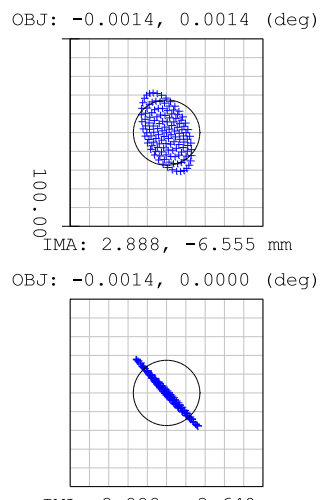

IMA: $2.888,-3.640 \mathrm{~m}$

OBJ $:-0.0014,-0.0014$ (deg)

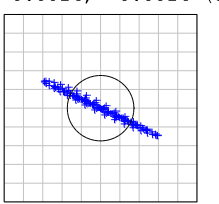

FP IMA: $2.889,-0.724 \mathrm{~mm}$

OBJ : $-0.0014,0.0014$ (deg)

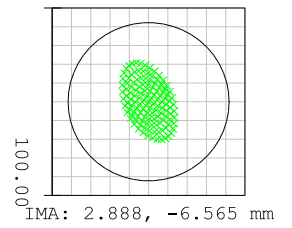

OBJ: $-0.0014,0.0000$ (deg)

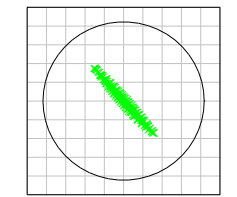

IMA: $2.888,-3.650 \mathrm{~mm}$

OBJ: $-0.0014,-0.0014$ (deg)

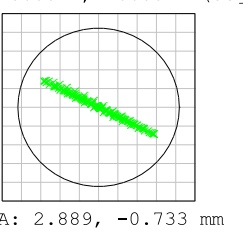

FP IMA : 2.889, $-0.733 \mathrm{~mm}$

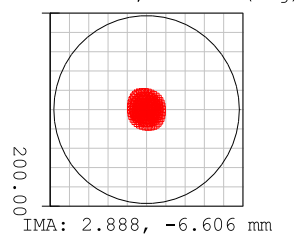

OBJ: $-0.0014,0.0000$ (deg)

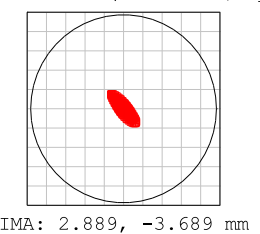

OBJ : $-0.0014,-0.0014$ (deg)

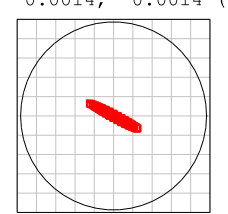

FP IMA: $2.889,-0.770 \mathrm{~mm}$

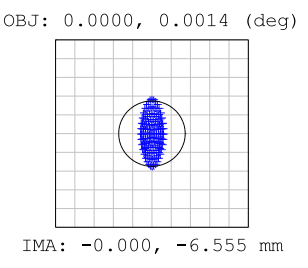

OBJ: $0.0000,0.0000$ (deg)
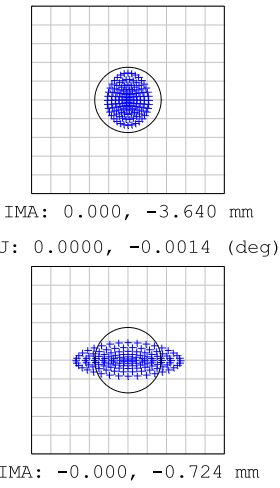

OBJ: $0.0000,0.0014$ (deg)

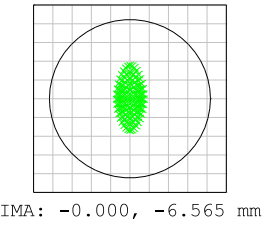

OBJ: $0.0000,0.0000$ (deg)

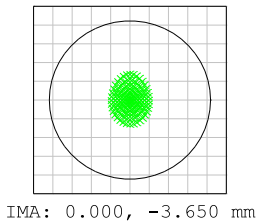

$\mathrm{BJ}: 0.0000,-0.0014$ (deg)

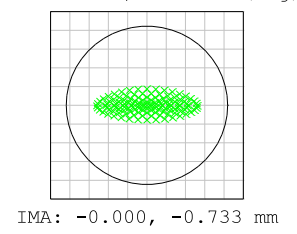

OBJ: $0.0000,0.0014$ (deg)

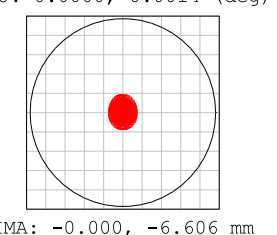

BJ: $0.0000,0.0000$ (deg)

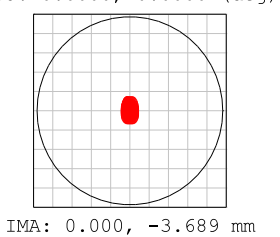

OBJ: $0.0000,-0.0014$ (deg)

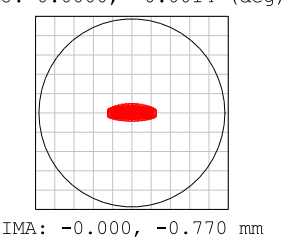

OBJ $: 0.0014,0.0014$ (deg)

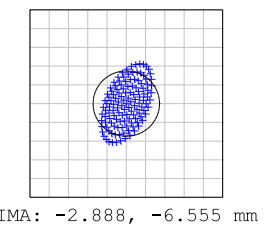

OBJ: $0.0014,0.0000$ (deg)

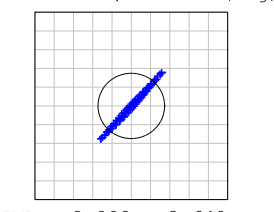

OBJ: $0.0014,-0.0014$ (deg)

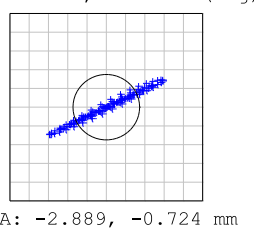

OBJ: $0.0014,0.0014$ (deg)

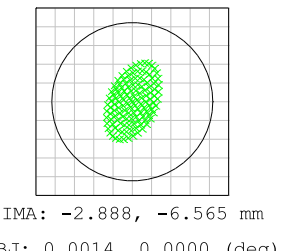

OBJ: $0.0014,0.0000$ (deg)

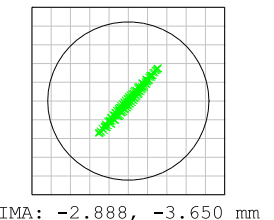

OBJ: $0.0014,-0.0014$ (deg)

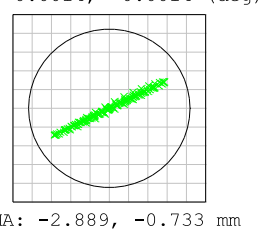

OBJ: $0.0014,0.0014$ (deg)

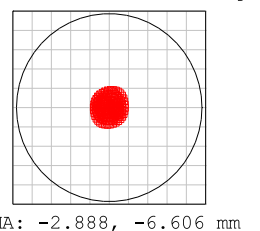

OBJ: $0.0014,0.0000$ (deg)

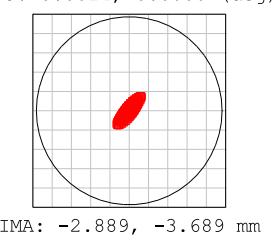

OBJ: $0.0014,-0.0014$ (deg)

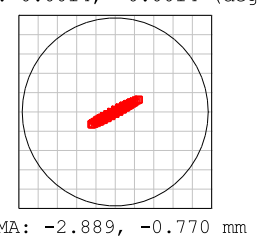


Figure 12.13.: (on page 154:) Spot diagrams at the slit across a $10^{\prime \prime} \times 10^{\prime \prime}$ field, at the average focus position. Note that the box size shown for each field point is $100 \mu \mathrm{m}$ for $\lambda=0.95 \mu \mathrm{m}$ (blue, at top) and $2.3 \mu \mathrm{m}$ (green, middle), and $200 \mu \mathrm{m}$ for $5.3 \mu \mathrm{m}$ (red, at bottom). The system is diffraction limited in almost all cases across the $10^{\prime \prime} \times 10^{\prime \prime}$ field shown.
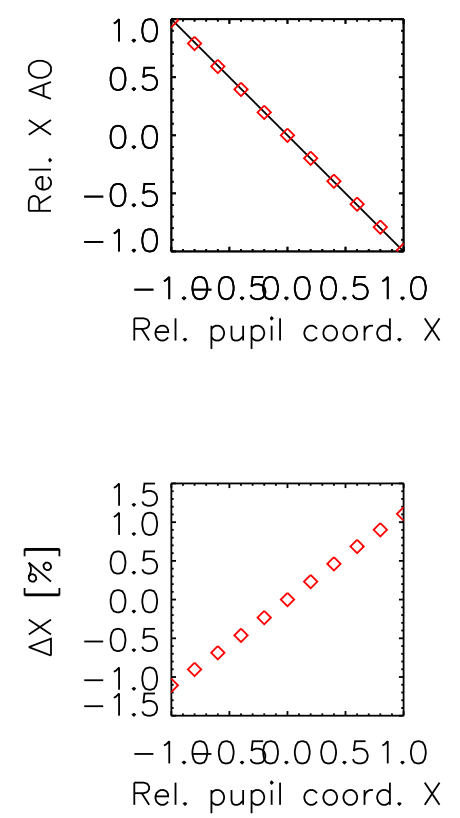
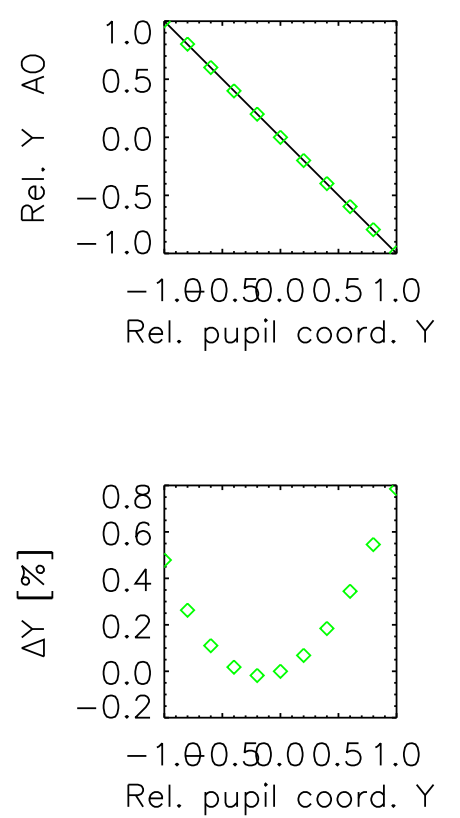

Figure 12.14.: (left:) Correspondence between the telescope pupil and the pupil image on the AO-DM. Imaged points on the AO-DM (in mm from centre, the diameter is $60 \mathrm{~mm}$ ) as a function of the originating coordinates in the pupil plane (relative coordinates) in $X$ and $Y$ directions (left and right panels, respectively). Note that the pupil image is flipped in both ways. (right:) Filling of the AO-DM aperture.

with respect to the entrance pupil plane. These measures will be important to facilitate a comparison with the effects of NAGICS in the path.

\section{Reflectance and throughput}

The CRIRES pre-slit optics feature a total of eight surfaces between the telescope and the image on the slit. There are six mirrors, the deformable mirror, and one window, plus the dichroic (which is coated on the window front). To estimate the optical losses due to imperfect reflectance and transmission, the following is assumed: the derotator mirrors and the AO mirrors are coated with protected silver, the $\mathrm{CaF}_{2}$ window is coated with an anti-reflective (on the backside). For the telescope mirrors, and for the AO-DM, protected aluminum coating is assumed.

Instead of the general idealistic coating with a uniform reflectivity for all wavelengths, I analyze the throughput across the entire system with laboratory measured reflectance data. This treatment allows for a wavelength dependent diagnostic of losses, which is deemed useful for two reasons. First, the behaviour is quite different over the $1-5 \mu \mathrm{m}$ NIR range, depending 
on the metal employed as a mirror coating. We will see for NAGICS, how the choice of coating defines the overall input efficiency. Second, the light dedicated for the AO $(<950 \mu m)$ must be considered as well. This is indicative for the AO-performance, and efficiency losses in this domain have a strong impact on the usability of the AO functionality.

Measured reflectance data ${ }^{6}$ have been obtained from Thorlabs for protected aluminum, silver, and gold, each for two angles of incidence (AOI; close to zero, and $45^{\circ}$ ). At high AOI, the reflective behaviour is significantly different, and also depends on polarization. The AOI is taken into account in the analysis, albeit at the very small field angles (ie. FOV) the resulting effect is weakly dependent on AOI. The exact behaviour of the real (time-dependent) coatings of VLT and warm-optics may be different from the assumptions here, but my interest is not in the absolute efficiency of the entire system (this severely depends on other constraints, such as aging), but in the relative, wavelength dependent efficiency. NAGICS, introducing a number of additional surfaces, also relies on a defined setup for the optical train in common. The data for $\mathrm{Al}, \mathrm{Ag}$, and $\mathrm{Au}$ are shown in Fig. 12.15 for completeness. Only the average reflectance for an AOI around zero is plotted. The $\mathrm{Al}$ data is lower by about $0.5 \%$ from $2 \mu \mathrm{m}$ on than the reflectance reported for the VLT mirrors by Ettlinger et al. (1999). Their measurements, however, do not extend beyond $2.5 \mu \mathrm{m}$. All three metal coatings have generally very high reflectance throughout the NIR ( $\mathrm{Ag}$ and $\mathrm{Au} \sim 98 \%, \mathrm{Al} \sim 95 \%$ ), but partly drop towards the visible $(\lesssim 1 \mu m)$.

The results of the analysis are shown in Fig. 12.16. Given the assumptions and properties of the coatings above, the system without gas cell maintains a high, common throughput from $2-5.3 \mu \mathrm{m}$, where about $60 \%$ of the initial energy arrives at the image plane on the slit. There is a strong decrease below $2 \mu \mathrm{m}$, with a minimum of $25 \%$ at $\sim 0.8 \mu \mathrm{m}$, owing to the reflectance break-down of Aluminum, and (more weakly) of Gold. Following the calculation, the minimum throughput in terms of light that passses through the slit is achieved at the CRIRES blue edge, at $0.95 \mu \mathrm{m}$ with $35 \%$ energy throughput. For lower wavelengths, note again that the dichroic prohibits transmission through the window and onto the slit, but reflects these wavelengths to the WFS. The dichroic is not modelled here as it has negligible absorption (thus, transmission plus reflectance match 100\%), instead I let all wavelengths pass through the window. The $\mathrm{CaF}_{2}$ window, however, does not suffer from a decreased transmission shortly below $1 \mu \mathrm{m}$, so the attenuation by the window is homogenously distributed over the entire wavelength range, and does not contribute to the particular loss of throughput below $1 \mu \mathrm{m}$.

The AO-WFS is most sensitive in the $R$-band $\left(\lambda_{c} \sim 0.7 \mu m\right)$, and so is especially hampered by the below-average performance of the preceeding mirror surfaces. Coatings (and the dichroic) must be chosen carefully when introducing further surfaces along the optical train, in order to keep the impair on the AO small.

\subsection{Baseline design performance analysis}

A complete optical model of the NAGICs baseline design is established following the design parameters derived in the previous section. The model comprises 14 surfaces, with corresponding curvature parameters, conics, distances, tilts, diameters, and coatings, and is embedded into the CRIRES model. This model is solved to meet all relevant requirements, and to work over the defined NIR wavelength range $0.95-5.3 \mu \mathrm{m}$, and for a minimum $10^{\prime \prime} \times 10^{\prime \prime} \mathrm{FoV}$.

\footnotetext{
${ }^{6}$ The Thorlabs coatings measured are G01 (protected Aluminum), P01 (protected Silver), and M01 (protected Gold). See http://www. thorlabs.com.
} 


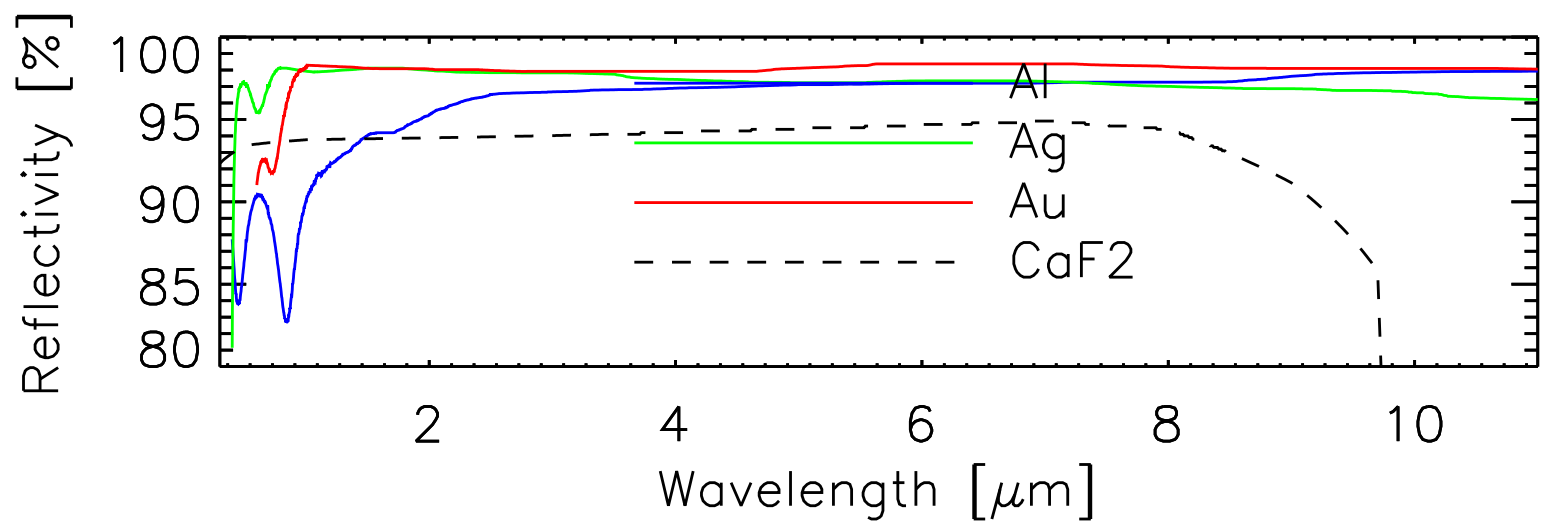

Figure 12.15.: Measured reflectance of HR metal coatings G01, M01, and P01 to estimate the surface losses of the warm-optics system. The transmission of $\mathrm{CaF}_{2}$ is also plotted (dashed line).

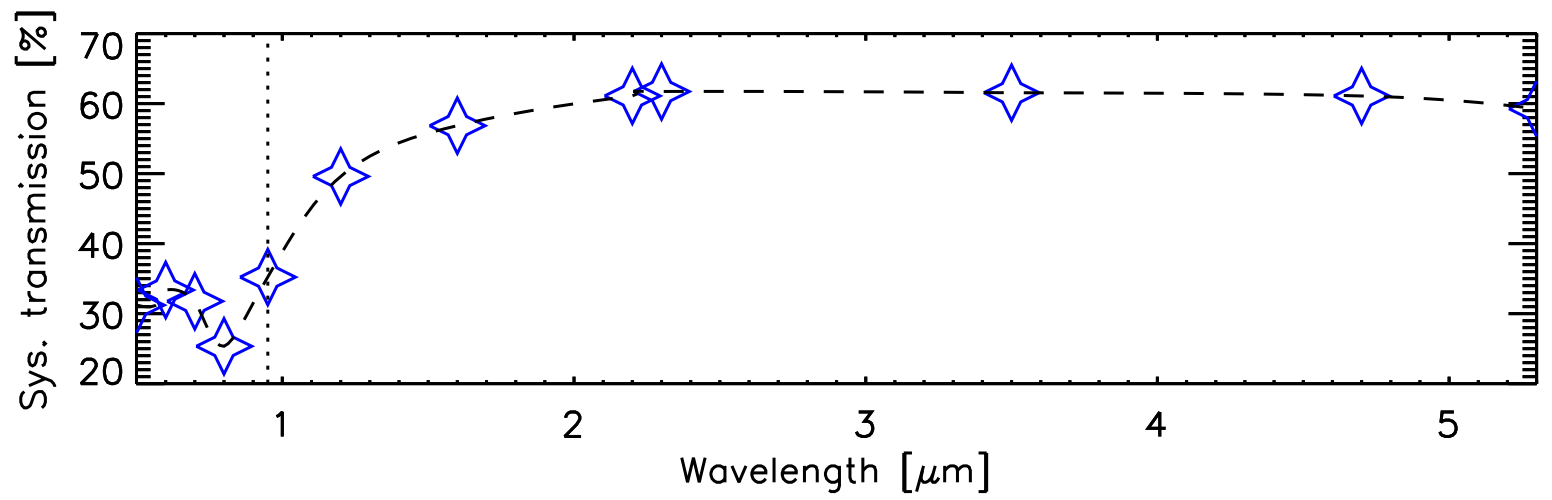

Figure 12.16.: Integrated system transmission of the VLT and the CRIRES warm-optics, based on wavelength dependent reflectance data for all mirror coatings, and the $\mathrm{CaF}_{2}$ window transmission. Note that $\lambda<0.95 \mu \mathrm{m}$ light is needed for the WFS of the AO system, and is reflected by a dichroic (vertical line).

The model is realized with a set of nearly 5800 conditions, for which a solution is computed. In this section, the converged solution is analyzed. To a large extent, the diagnostics are the same as employed for the measurements and analyses in Sec. 12.2.2 for the normal CRIRES with no calibration gas cell in the optical path.

In the following, an analysis of the optical capabilities is performed, followed by computations on the spectroscopic merit of the NAGICs calibration gas cell (ie. spectral imprints of selected calibration gases).

\subsubsection{Optical performance}

The optical performance of the NAGICS baseline design is characterized in several aspects. A comparison is then drawn between the system as described in Sec. 12.1 ("with NAGICs") and the system without NAGICS in the optical path as outlined in Sec. 12.2 ("no cell"). For the latter, the comparison is based mostly not on the full CRIREs FoV $\left(50^{\prime \prime} \times 50^{\prime \prime}\right)$, but on the same $10^{\prime \prime} \times 10^{\prime \prime}$ FoV as for NAGICS. 
The characterization of the NAGICS optical performance in this section comprises an evaluation of the usable FoV, the selection and corresponding impact of coatings on the system transmission, the chromatism introduced, and the image quality using various metrics. A verification to meet the requirements on scale and pupil is also performed.

\section{Field of view}

The NAGics baseline design provides a minimal FoV of $10^{\prime \prime} \times 10^{\prime \prime}$, mostly reasoned by limitations on the applicable mirror diameters (see Sec. 12.1.3). The FoV is defined by a field mask at the intermediate focus following the FO sub-system. If desired, the mask can be tailored to a larger FoV, at the cost of vignetting. The vignetting arises from the sizes of the FM units, which are determined following the baselined FoV. Close to the FP (or any IF), the FM units allow for a larger field than $10^{\prime \prime}$, but this is not the case for FM closer to the collimated beam. Thus, FM3-FM7 will quickly start to vignette for larger allowed fields than $10^{\prime \prime} \times 10^{\prime \prime}$.

The system's behaviour is shown in Fig. 12.17, where the percentage of vignetting, obtained from raytracing, is plotted against the diagonal field angle. The NAGICS system alone is by design free of vignetting until a diagonal field angle of $14^{\prime \prime}$ (see the blue dotted line). Fig. 12.17 also shows that the combination of NAGICS and warm-optics suffers from small amounts of vignetting from the start (solid blue line). This is caused by the undersized AO-DM unit, for which I assume a reflective $60 \mathrm{~mm}$ diameter. Note that the collimated beam on the AO mirror is not the very same with NAGICS, compared to no cell. It is compromised by the need to find a balanced solution that satisfies all relevant optical needs. If the AO-DM is enlarged (dotted blue line), the vignetting contribution by NAGICS becomes apparent. For comparison, the black dashed line shows the behaviour of the CRIRES model without calibration cell mechanism. Almost the entire $50^{\prime \prime} \times 50^{\prime \prime} \mathrm{FoV}$ is unvignetted, with a small $3.4 \%$ vignetting only for the outmost arcseconds (also due to the AO-DM).

\section{Coating efficiency}

The NAGICs baseline model is analyzed for its wavelength dependent transmission efficiency analogous to the calculations performed for the system without a gas cell device in Sec. 12.2.2. A major goal of this analysis is an estimate on the change of throughput, which unavoidably occurs when more optical surfaces are introduced into the system. Also, the impact of different coatings need to be assessed, thus enabling a coating selection for NAGICS. NAGICS features twelve mirror surfaces in the baseline design, plus a minimum of two glass windows for the captive gas vessel. For the windows, a thickness of $5 \mathrm{~mm}$ is baselined to be on the safe side, although $3 \mathrm{~mm}$ thicknesses might suffice. There are mostly two options for the mirror coatings $(\mathrm{Ag}$ and $\mathrm{Au}$ ), which have been discussed in Sec. 12.2.2. The remaining mirrors (and the cryostat entrance window) remain unchanged, as stated in the requirements. The fraction of energy lost due to imperfect reflectance (and transmission) is determined by integrating the losses for all surfaces, beginning with the telescope primary, until the first focal plane inside the cryostat (location of the entrance slit). Wavelength, incident angles and glass thickness are considered, based on the empirical data also presented in Sec. 12.2.2.

The dichroic layer is not specifically modelled, so all wavelengths are measured on the slit. This resembles an ideal step-function at $0.95 \mu \mathrm{m}$, the real behaviour is certainly a smeared out step-function (with high-frequency modulations), so that the real dichroic has an additional damping effect near it's cut-off wavelength, plus possibly a small general attenuation 


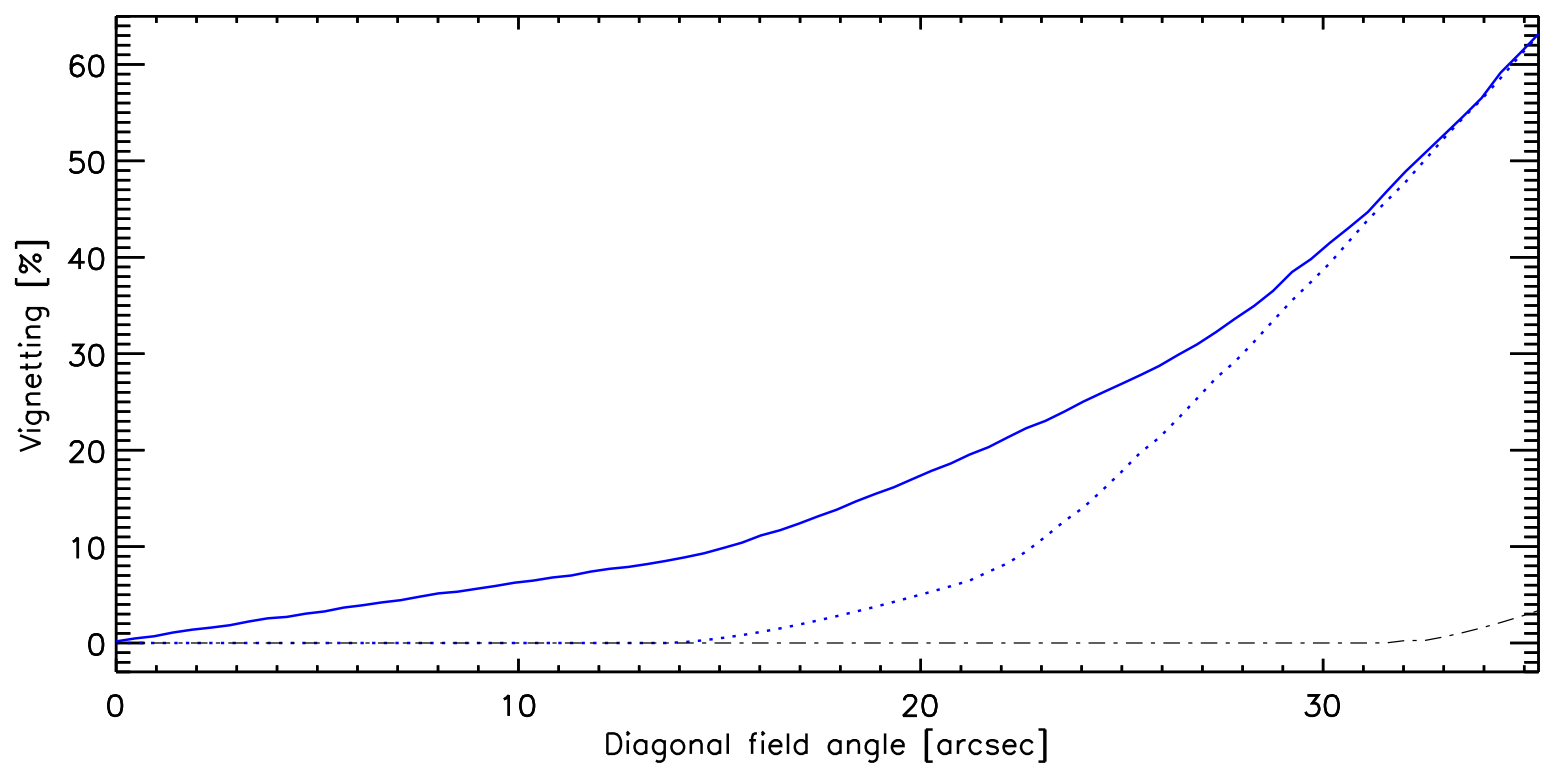

Figure 12.17.: System vignetting for off-axis field angles with NAGICs (blue lines) and without any cell in the optical train (black line). The normal CRIRES has an unvignetted FoV of almost the entire $50^{\prime \prime} \times 50^{\prime \prime}\left(\sim 34^{\prime \prime}\right.$ diagonal $)$. The maximum vignetting is only $3.4 \%$ at the very edge. For NAGICs, the baseline design FoV is $10^{\prime \prime} \times 10^{\prime \prime}$, with a resulting $6.2 \%$ at the edge (solid line). At larger off-axis angles, the level of vignetting steepens. For small fields, vignetting is solely contributed by the AO-DM. For comparison, the curve for NAGICs is shown (blue dotted line), when the AO-DM is not fixed to $60 \mathrm{~mm}$ diameter.

independent of wavelength. Again, the absolute transmission function as arriving on the slit is not of relevance here, as I focus on the relative changes due to NAGICS only.

The results for NAGiCS are summarized in Fig. 12.18. Three aspects are readily seen: First, the two glass windows baselined as entrance into and exit from the gas container vessel have a strong impact on the overall throughput. They reduce the transmission by about 10\%, with almost no dependence on wavelength. This means that the windows are reason for significant losses, even for the $\mathrm{CaF}_{2}$ glass with very favourable transmission (see Sec. 12.2.2). As a result for the vessel design, an interior solution where the vessel is accomodated inside the $\mathrm{RCRF}$, and where a total of ten window passages is required, is rendered unfeasible. This would translate in a factor of two in additional losses, and strongly impairs the usability of the whole spectrograph.

Second, the benefits of protected Gold coatings for all NAGICs mirror surfaces are small. The strengths of Gold come into play at wavelengths $\lambda>4 \mu m$, with a gain over Silver of about $5 \%$. Shortwards, the Silver coatings are equally well suited, or better $(\lambda<2 \mu m)$, resulting in a boost of $\sim 9 \%$ at $1 \mu \mathrm{m}$ over Gold. Third, the choice of coating has a severe impact on the AO performance. A difference of $34 \%$ gain at $0.8 \mu \mathrm{m}$ in favour of Silver advocates against a Gold coating, given that the peak AO efficiency relies on the the $R$-band region.

The overall performance in terms of throughput as seen on the instrument focal plane (where the CRIRES+ slit is located) is, on average, a factor of $1.54-1.82$ loss compared to NAGICS taken out of the path, depending on wavelength (Silver coatings assumed). As a result, the overall observing efficiency of CRIRES (neglecting other changes/improvements 

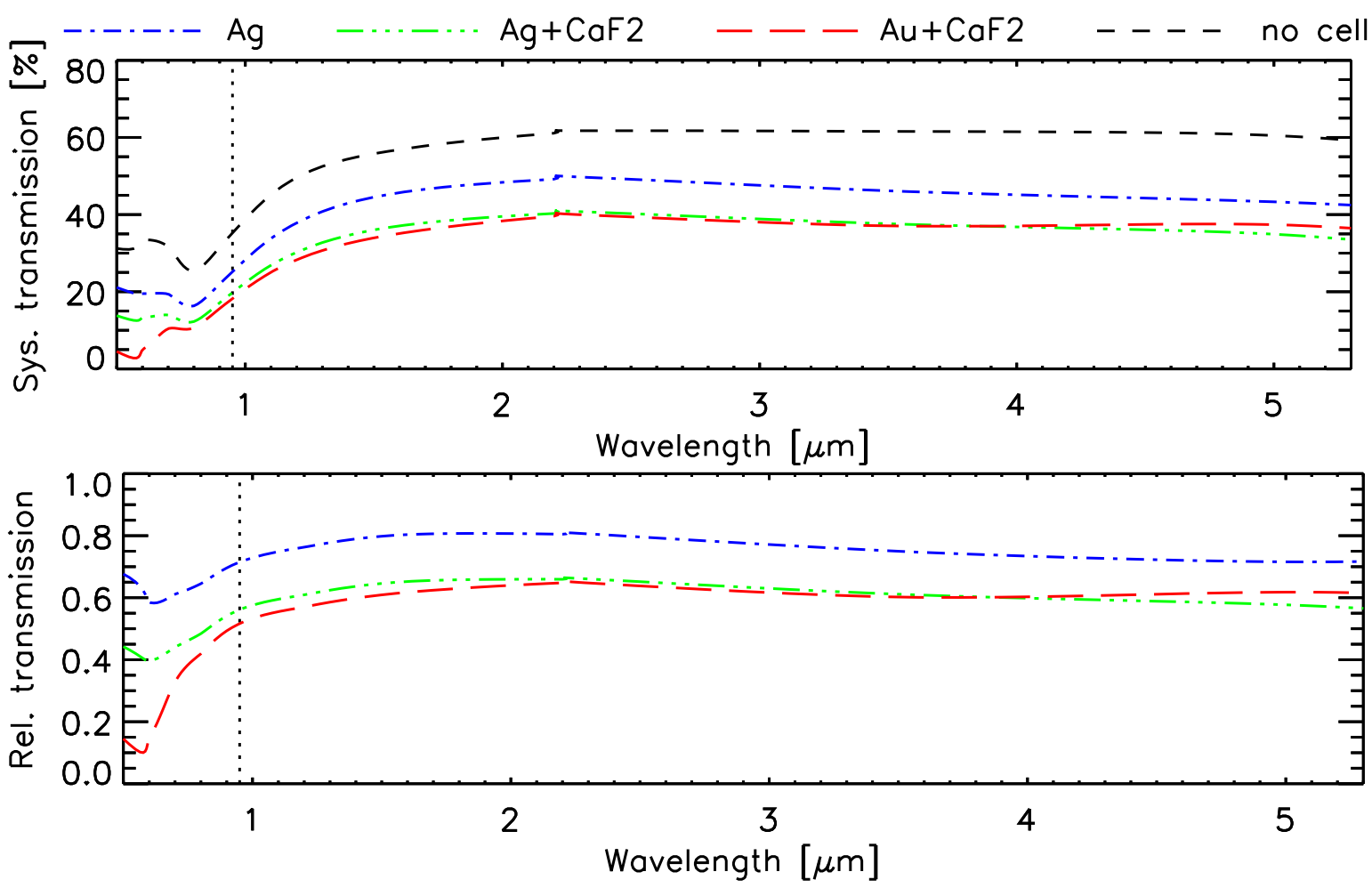

Figure 12.18.: (top:) Integrated system transmission of the VLT and the CRIRES warmoptics, with NAGICS in place. Calculations are performed for the VLT plus CRIRES and NAGICS model, with either protected Silver coatings or protected Gold coatings for the NAGics mirrors. Two $\mathrm{CaF}_{2}$ windows are in place and taken into account. For comparison, the system transmission for Silver coatings, but without the two NAGICS windows, is also shown (blue dashed dotted line). The black dashed line resembles the system without NAGICS. Note that $\lambda<0.95 \mu \mathrm{m}$ light is needed for the WFS of the AO system, and is reflected by a dichroic (vertical dotted line).

(bottom:) Relative transmission performances normalized to the "no cell" system (CRIRES without NAGICS; cf. Sec. 12.2.2).

made to the instrument) will be downshifted by $0.5-0.7$ magnitudes; ie. the current efficiency will be achieved for stars $0.5-0.7 \mathrm{mag}$ brighter, over the entire wavelength regime. The AO performance is reduced by a factor of 2.2 , so that the $\mathrm{AO}$ guide star limit can be estimated to decrease by 0.9 magnitudes (albeit this strongly depends on the star's color).

All following analyses are based on the baseline design, with protected Silver ceated NAGICS mirrors, and $5 \mathrm{~mm} \mathrm{CaF}_{2}$ windows (unless otherwise stated).

\section{Image scale}

The nominal image scale at the Nasmyth FP is not constant, as a result of field curvature. Near the field centre, it is $582 \mu \mathrm{m} /$ arcsec. As the AO-relay has unit magnification, the image scale at the CRIRES + slit FP is the same, in first order (the pre-slit relay of the current CRIRES is discontinued). Inserting the multi-mirror device NAGICS will introduce small deviations in the image scale, although the baseline design is driven by the need to maintain unit 

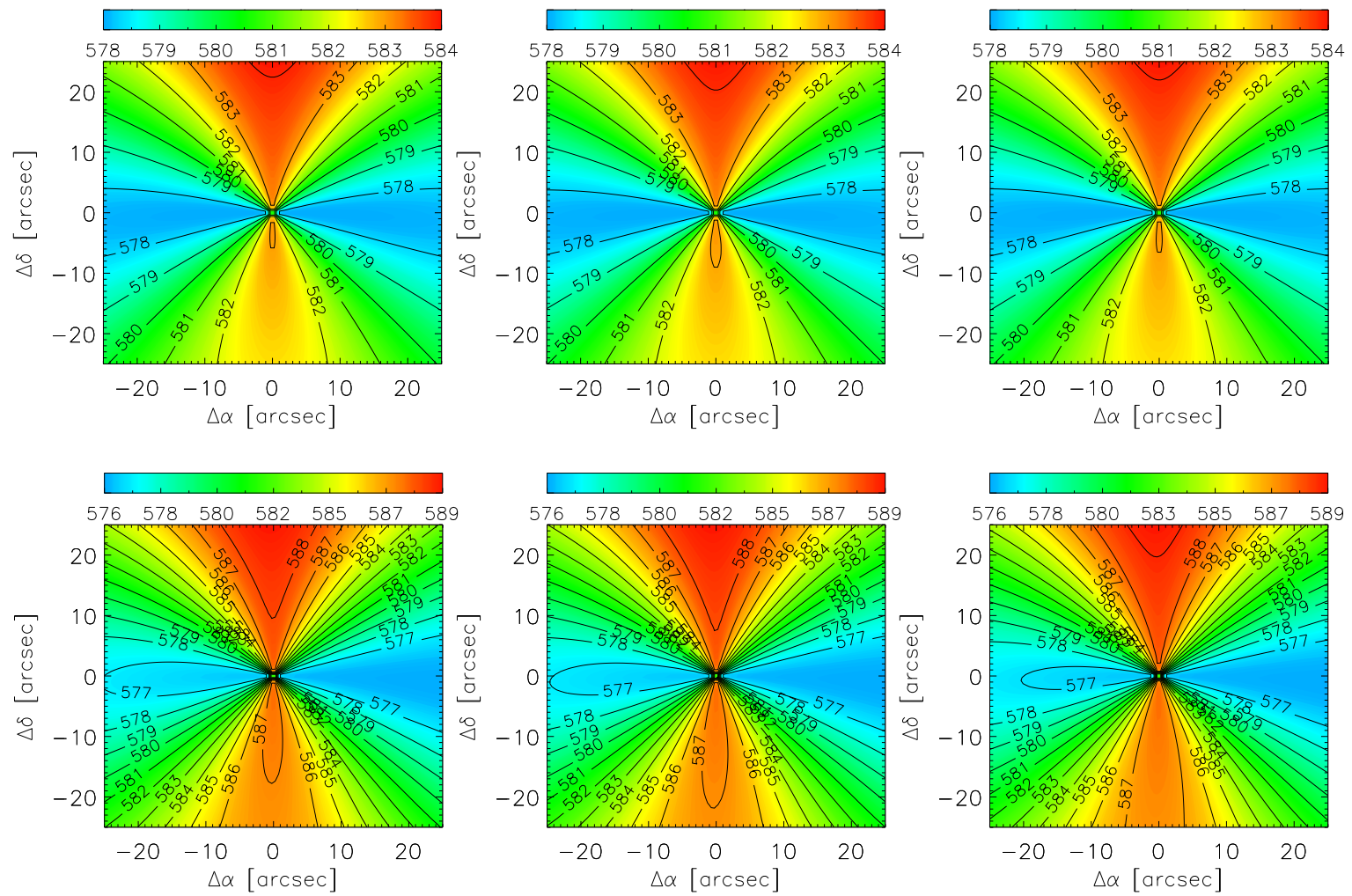

Figure 12.19.: Contour maps of the image scale across the slit FP without cell (top panels), and with NAGICS (bottom panels). The image scale is given in $\mu \mathrm{m} / \operatorname{arcsec}$, for $x, y$ offsets (in projected sky angle) around the on-axis point. Three wavelengths are plotted, $0.95 \mu \mathrm{m}, 2.3 \mu \mathrm{m}$, and $5.3 \mu \mathrm{m}$ (panels from left to right, respectively). Note that the baselined NAGiCs FoV is only $10^{\prime \prime} \times 10^{\prime \prime}$, at larger angles vignetting occurs.

magnification. To characterize the NAGICs image scale, I measure the scale factor across the slit FP on a grid of points, and compare it to the results obtained in the same way without the cell. The image scale is determined by the object sky angle around the on-axis field, and the resulting centroid position in the FP of the corresponding point source. Fig. 12.19 summarizes the results of the analysis as contour maps for the configuration with, and without NAGICS. Without cell (in the top panels), the image scale shows a radial-symmetric structure, with little deviation along the $\alpha$ and $\delta$ axes (with smaller and larger scales than average along the axes, respectively). This corresponds to a slightly larger scale along the slit $\left(\sim 583 \mu \mathrm{m} /{ }^{\prime \prime}\right)$ than perpendicular to the slit $\left(\sim 578 \mu \mathrm{m} /{ }^{\prime \prime}\right)$. A very small wavelength depence of the image scale (negligible) can be observed in the $0.95 \mu \mathrm{m}, 2.3 \mu \mathrm{m}$, and $5.3 \mu \mathrm{m}$ maps (left to right in Fig. 12.19).

The overall structure of the image scale is the same when NAGICS is placed into the optical train. The PTV amplitude is larger $\left(\sim 576-589 \mu \mathrm{m} /{ }^{\prime \prime}\right)$, so that the average scale factor along the slit is now $\sim 577 \mu \mathrm{m} /{ }^{\prime \prime}$ (the FP is rotated $90^{\circ} \mathrm{CCW}$ with NAGICS), and $\sim 587 \mu \mathrm{m} /{ }^{\prime \prime}$ perpendicular to it. The wavelength dependence persists (due to the entrance window), but is still negligible.

Thus, by design NAGICS maintains the magnification of the CRIRES warm-optics to within $0.8 \%$. 


\section{Field dependent Strehl ratios}

One of the design goals for NAGICS is to deliver an image quality at the CRIRES slit that is not compromised. A direct comparison of the performances for the CRIRES warm-optics alone, and including NAGICs is presented in Figs. 12.20 and 12.21 in terms of the achieved Strehl ratios $s$ (see also Fig. 12.8). While Fig. 12.20 represents contour maps of the whole plane of the field, Fig. 12.21 shows cuts of the maps along the axes, for the three wavelengths simultaneously.

For the envisioned $10^{\prime \prime} \times 10^{\prime \prime}$ FoV, NAGICS outperforms the unmodified configuration at all operating wavelengths, and across the entire field. This performance boost with NAGICS, despite of the massive beam massaging by the RCRF sub-system, is a result of the highly optimized RCRF, which is partly capable of compensating the AO-mirrors' deficiencies. The improvement provided by NAGICS amounts to a gain of $11 \%$ in Strehl ratio at the shortest wavelengths (where the current spectrograph is least efficient) around the central on-axis field (see Fig. 12.21). The advantage falls off towards the field corners $(\Delta s=9 \%$ ), and becomes smaller at longer wavelengths. At $5.3 \mu \mathrm{m}$, the difference is not noticable over the employed FoV, so the NAGICS performance is still on par with the system and no cell, despite the numerous limitations imposed on the optical design, and the two additional refractive elements (windows).

NAGICS effectively maintains diffraction limited performance $(s \geq 0.8)$ over its full FoV, for all but the bluest wavelength range.

\section{Chromatic focal shift}

The chromatic focal shift in the FP does not remain unchanged with NAGICS, compared to Sec. 12.3.1. Two shifts arise, a lateral displacement in the FP, depending on wavelength, and an axial displacement with a slightly offset position of the FP along the OPX, for each wavelength.

Axial shift: besides the tilted entrance window as a major source of the shift, two more windows (not tilted) are baselined. Their contributions (due to small deviations in incident angle on the windows, across the beam diameter) add to an increased axial focal shift. As with no cell inserted, this shift is both a function of wavelength and of off-axis position in the FP. For the on-axis case and the FoV corner field point, the shifts are computed in Fig. 12.22 for the CRIRES wavelength range. With NAGICS, the total shift on-axis is $300 \mu \mathrm{m}$ between $0.95 \mu \mathrm{m}$ and $5.3 \mu \mathrm{m}$, and $340 \mu \mathrm{mat}$ off-axis. This compares to $140 \mu \mathrm{m}$ (on-axis) and $160 \mu \mathrm{m}$ (at the same $10^{\prime \prime} \times 10^{\prime \prime}$ field position) for the un-modified CRIRES warm optics.

The absolute focus position also changes when NAGICS is inserted, by about 200-240 $\mu \mathrm{m}$ (depending on field position). This makes a refocussing mandatory when the cell is inserted, by means of the telescope M2 focus stage.

Lateral shift: the wavelength dependent image location in the FP is caused by the tilted entrance window, and does not change. The displacement (with or without NAGICS) is $\sim 44 \mu \mathrm{m}$ on-axis between $0.95 \mu \mathrm{m}$ and $5.3 \mu \mathrm{m}$, and increases slightly towards the field edges (see Fig. 12.9).

\section{Encircled energy fractions}

The optical performance of NAGICS is further assessed by the fraction of encircled energy within a given radius. It is important to realize that the fractions of encircled energy shown here are for the reduced $10^{\prime \prime} \times 10^{\prime \prime} \mathrm{FOV}$, whereas in Sec. 12.2.2 the aim was to verify the 

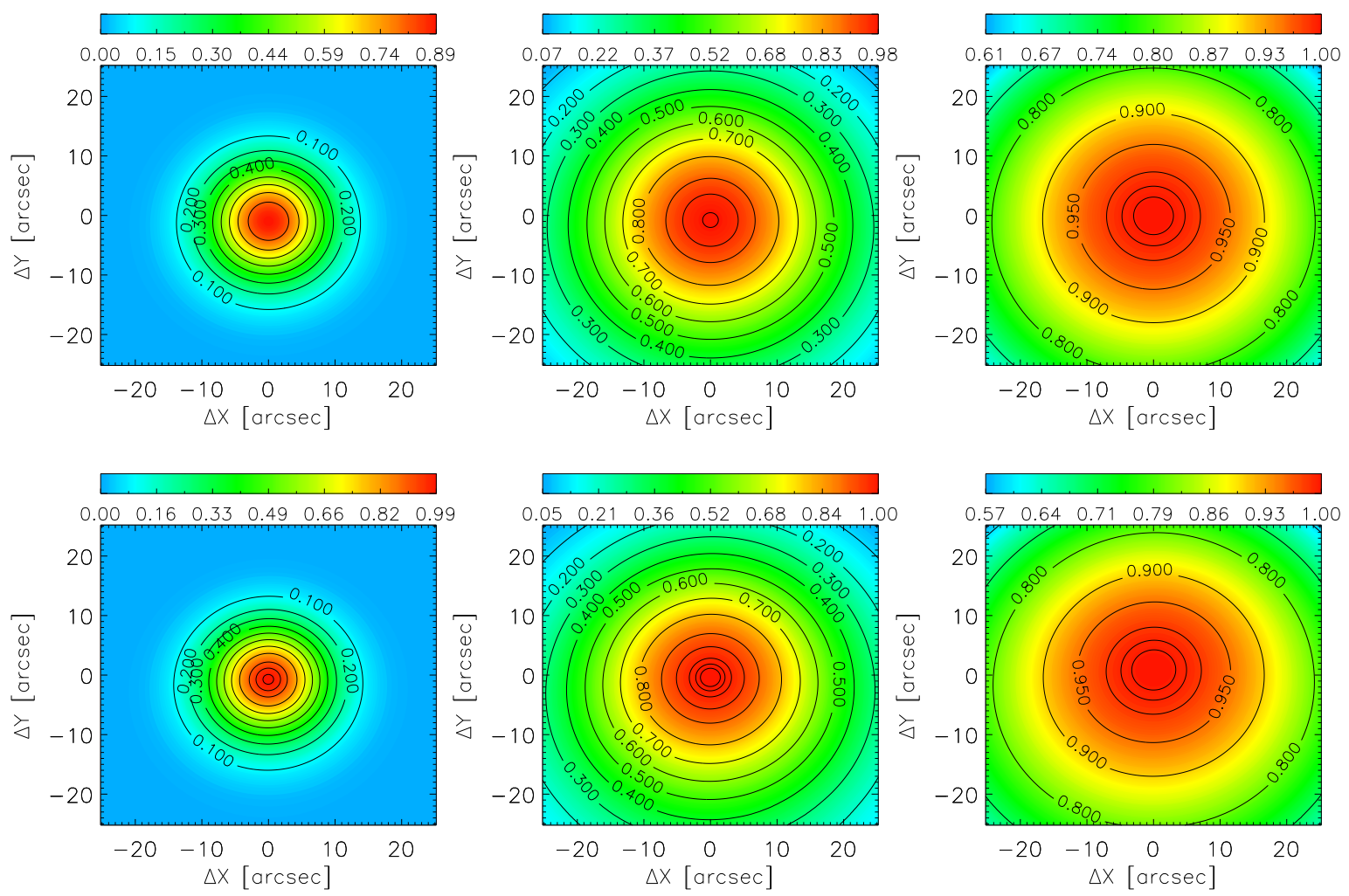

Figure 12.20.: Maps of Strehl ratios as a function of position around the on-axis field. Top row panels for no cell inserted, bottom row panels with NAGICS. Three wavelengths are plotted, $0.95 \mu \mathrm{m}, 2.3 \mu \mathrm{m}$, and $5.3 \mu \mathrm{m}$ (panels from left to right, respectively). NAGICS achieves a higher Strehl at all wavelengths, with the largest improvement at the shortest wavelength.

performance of the full CRIRES FoV. Fig. 12.24 shows the performance when NAGICS is inserted into the optical path (thick lines). For comparison, the performance of the "no cell" configuration is overplotted as thin lines, for the same field points. The encircling energy capabilities with NAGICS are virtually the same as without. This proves the very high design performance at all wavelengths over the envisioned FoV. In the blue regime, the performance is actually slightly improved by at most $10 \%$ (at all field angles), but the advantage weakens by a radial distance of $0.05^{\prime \prime}$, and is there almost negligible at longer wavelengths. At $2.3 \mu \mathrm{m}$ the $80 \%$ energy level, as an example, is achieved within $29.66 \mu \mathrm{m}$ (on-axis) and $35.12 \mu \mathrm{m}$ (worst off-axis) in FP space with NAGICS. This compares to $30.52 \mu \mathrm{m}$ and $37.63 \mu \mathrm{m}$, respectively, without the cell device. The theoretical, purely diffraction limited distance is $29.61 \mu \mathrm{m}$ at $2.3 \mu \mathrm{m}$. Fig. 12.25 plots the relative encircled energy fractions, normalized to the "no cell" configuration, as a function of radial distance from the corresponding field points.

The fact alone that the performance with NAGICS is not inferior, in terms of encircled energy, is remarkable and a result of the highly optimized RCRF mirrors, that mildly correct for the aberrations of the (inactive) AO system. Note that the encircled energy fractions are relative to the total energy enclosed, which arrives at the FP. As such, Figs. 12.24 and 12.25 cannot reflect the additional transmission losses introduced with NAGics (see Fig. 12.18). 

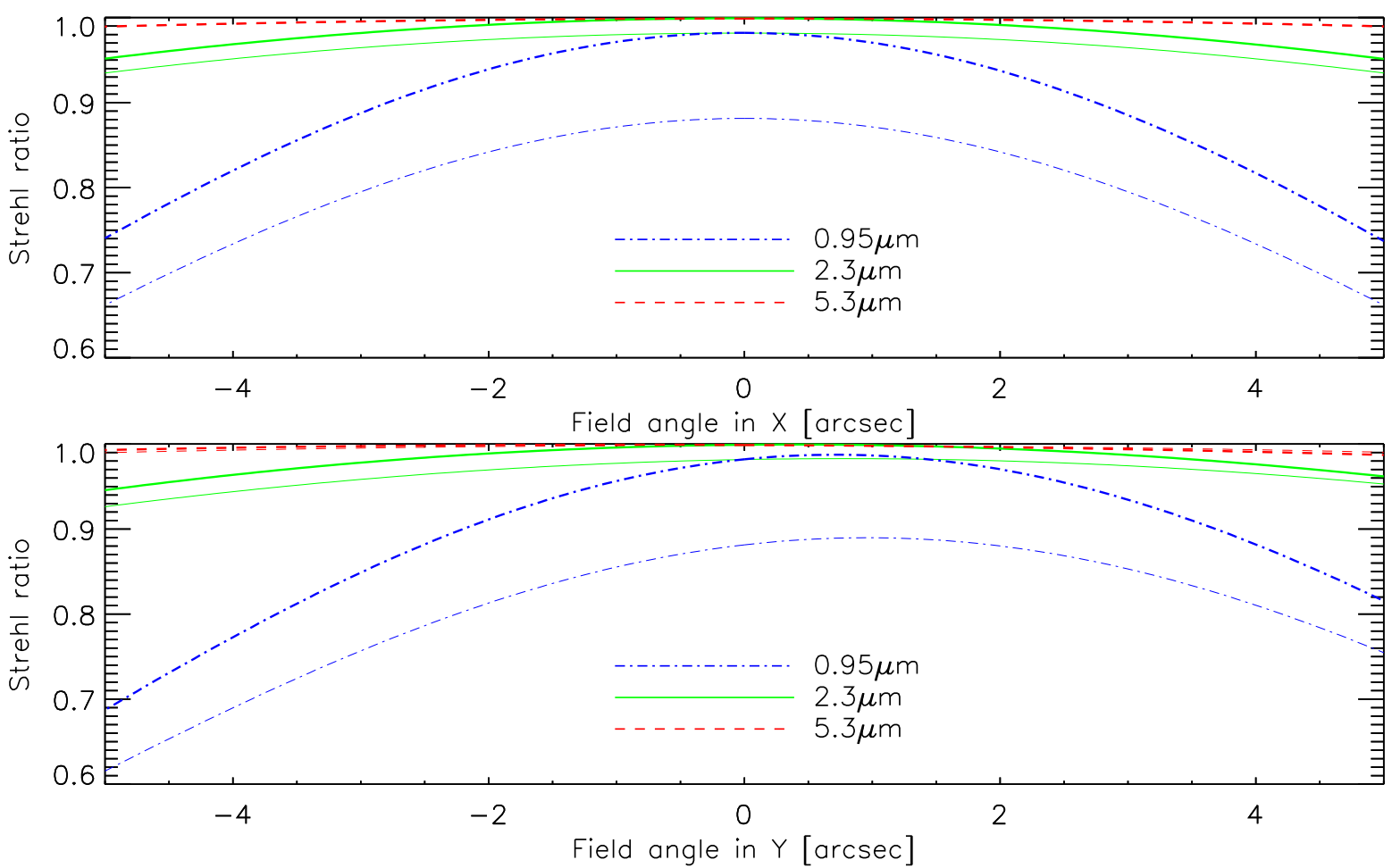

Figure 12.21.: Comparison of Strehl ratios as a function of field angle around the on-axis position. The X-cut (top) and Y-cut (bottom) are plotted along the $10^{\prime \prime} \times 10^{\prime \prime}$ field, for the three usual wavelengths $0.95 \mu \mathrm{m}, 2.3 \mu \mathrm{m}$, and $5.3 \mu \mathrm{m}$ (blue, green, and red, respectively). Thick lines represent the performance achieved with NAGICS, thin lines with no cell inserted.

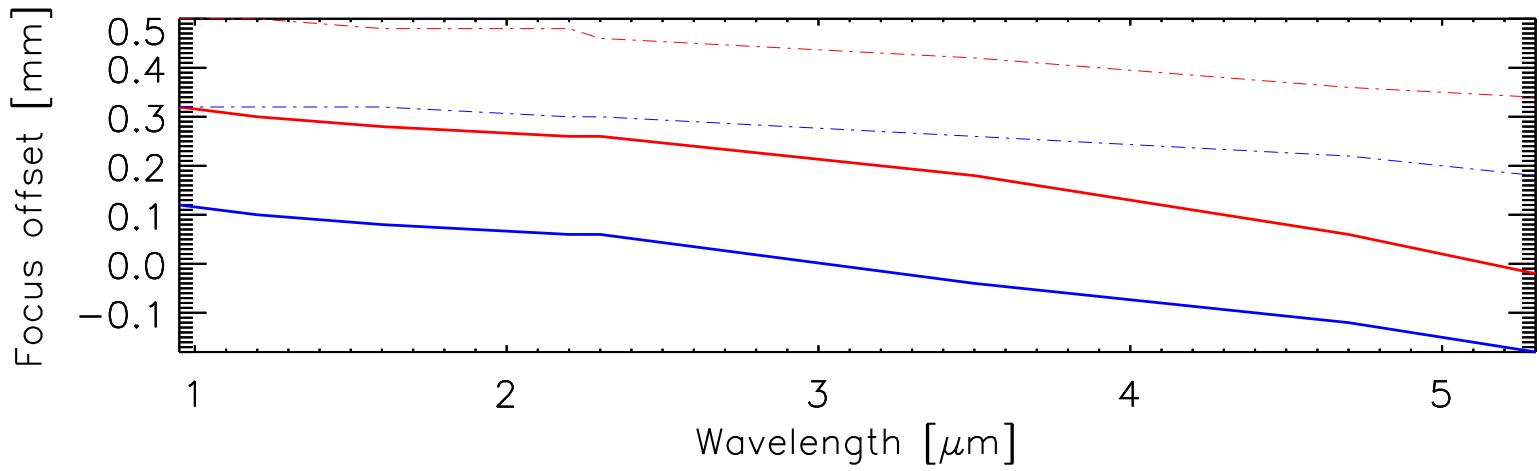

Figure 12.22.: Wavelength dependent axial focal shift, plotted for NAGICs (thick lines) and for no cell (thin dashed lines). In both cases, the on-axis points (blue) and the $10^{\prime \prime} \times 10^{\prime \prime}$ field edge (red) behave slightly different with wavelength. The effect is due to the wavelength sensitive dispersion of the window material. NAGICS features two additional windows.

\section{Spot sizes in the image plane}

The image quality on the CRIRES+ entrance slit is also adressed by the capabilities of the combined system (VLT, derotator, AO-module, entrance window, plus NAGICS) to image 

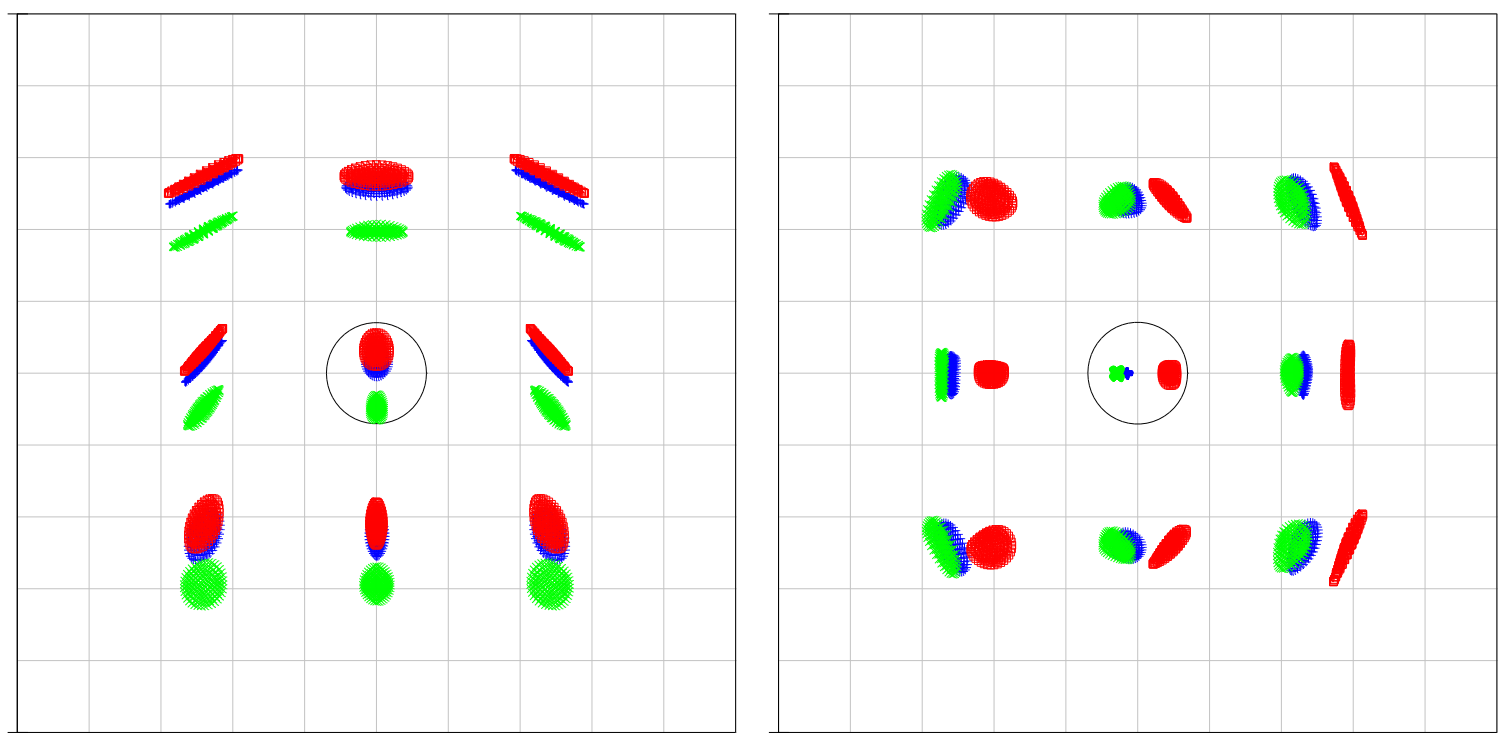

Figure 12.23.: Lateral wavelength dependent displacement at the instrument FP (at the CRIRES+ slit). The spot distributions mark the on-axis and boundary field points of the $10^{\prime \prime} \times 10^{\prime \prime} \mathrm{FoV}$ (the FP shown covers $12 \mathrm{~mm}$ box length). The size of the spot distributions is exaggerated by $\times 20$ and given by the $42 \mu \mathrm{m}$ radius of the Airy disk (circle, for $2.3 \mu \mathrm{m})$.

Colours represent $\lambda=0.95,2.3$, and $5.4 \mu \mathrm{m}$ (green, blue, and red, respectively). (left:) With no cell inserted. The entrance slit is in the horizontal direction. (right:) With NAGICs inserted. The entrance slit is in the vertical direction, rotated $90^{\circ} \mathrm{CCW}$ with respect to no cell (cf. Fig. 12.10).

point sources onto the FP. The sizes of point sources distributed over the $10^{\prime \prime} \times 10^{\prime \prime} \mathrm{FoV}$ depends on wavelength, as previously discussed. I plot the RMS sizes in the FP at the CRIRES+ slit in Fig. 12.26, and their geometric distributions in Fig. 12.27. Fig. 12.26 presents a direct comparison between NAGICS (plotted in blue) and the un-modified CRIRES warmoptics (plotted in black). The spot sizes are given as the RMS radii relative to the centroid positions of the corresponding spot images, and evaluated for the whole range of operating wavelengths. Three different radii are determined, to account for the uneven (elongated) shape of the spots. The solid lines are RMS radii, dotted lines resemble RMS size in Xdirection only, and dashed lines represent the RMS size in Y-direction. A representation of the spot shapes is depicted in Fig. 12.27 for the case of NAGICs.

The performance of the NAGICS baseline design is superb, and renders the system diffraction limited at all wavelengths, and across the entire field. When compared to the setup without the cell device inserted, the image quality is not compromised due to NAGICs. On the contrary, NAGICS improves the performance by a factor of up to seven. This is most notable on-axis, where the overall smallest spot size is achieved at $\lambda=3 \mu \mathrm{m}$. The improvement generally becomes smaller at longer wavelengths and towards the field corners. At one edge of the FoV, there is a slight degradation for $\lambda>3 \mu \mathrm{m}$, owing to the much elongated shapes of the point images. In any case, the practically relevant diffraction limit, which scales with $\lambda$, implies an Airy disk that is larger than the obtained spot radii in all cases (included in Fig. 12.27. 

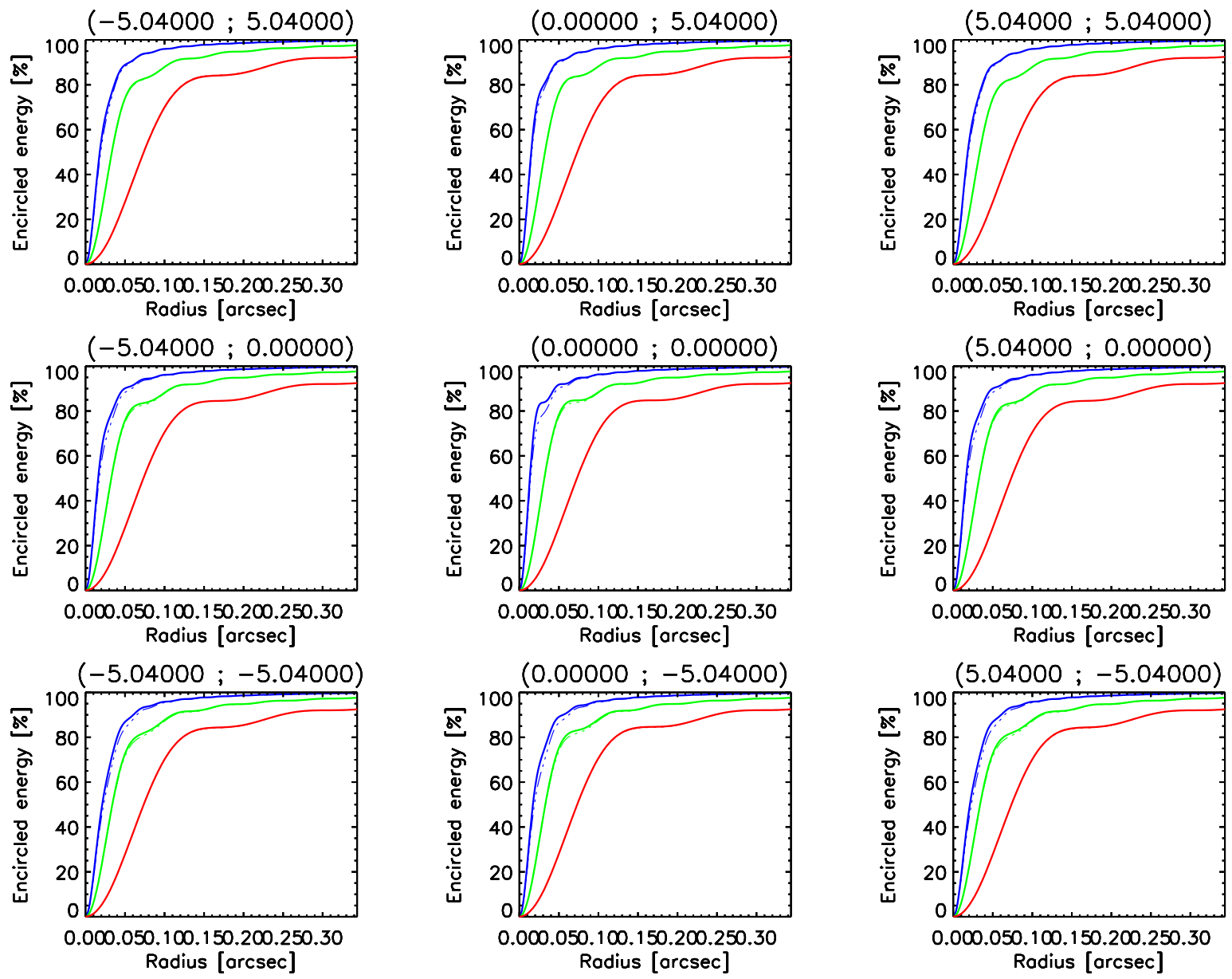

Figure 12.24.: Encircled energy fractions with NAGICS, at the slit. The energy fractions are computed for the wavelengths $0.95 \mu \mathrm{m}, 2.3 \mu \mathrm{m}$, and $5.3 \mu \mathrm{m}$ (blue, green, red, respectively), for 9 field points in the $10^{\prime \prime} \times 10^{\prime \prime} \mathrm{FoV}$ (coordinates $x, y$ in $\operatorname{arcsec}$ around the on-axis field). The slit orientation is along the middle column. For a slit width of $0.2^{\prime \prime}\left(R=10^{5}\right)$, an ideal target at the slit center passes $\sim 100 \%$ and $85 \%$ of the energy into the spectrograph at $0.95 \mu \mathrm{m}$ and $5.3 \mu \mathrm{m}$, respectively (no atmospheric seeing assumed). There is virtually no degradation compared to the "no cell" results (plotted as thin dash-dotted lines).

Thus, NAGICs is capable of maintaining the high image quality and diffraction limited performance (without active AO) of the system as without a calibration gas cell.

\section{Pupil imaging on AO-DM}

The requirement of pupil imaging onto the AO-DM has strong implications on the NAGICS image quality, and is a limiting factor when searching the optimal solution for the baseline design parameters. This is not a surprise, as NAGICS must both image object and pupil plane under the constraints of fixed relay optics and focal plane position. Thus, both the pupil image plane, and the image plane must be placed in the same way as if there was no NAGICS.

A compromise solution between best possible image quality at the FP, and the best achievable pupil placement on the AO-DM has been chosen. The results for the pupil image are 

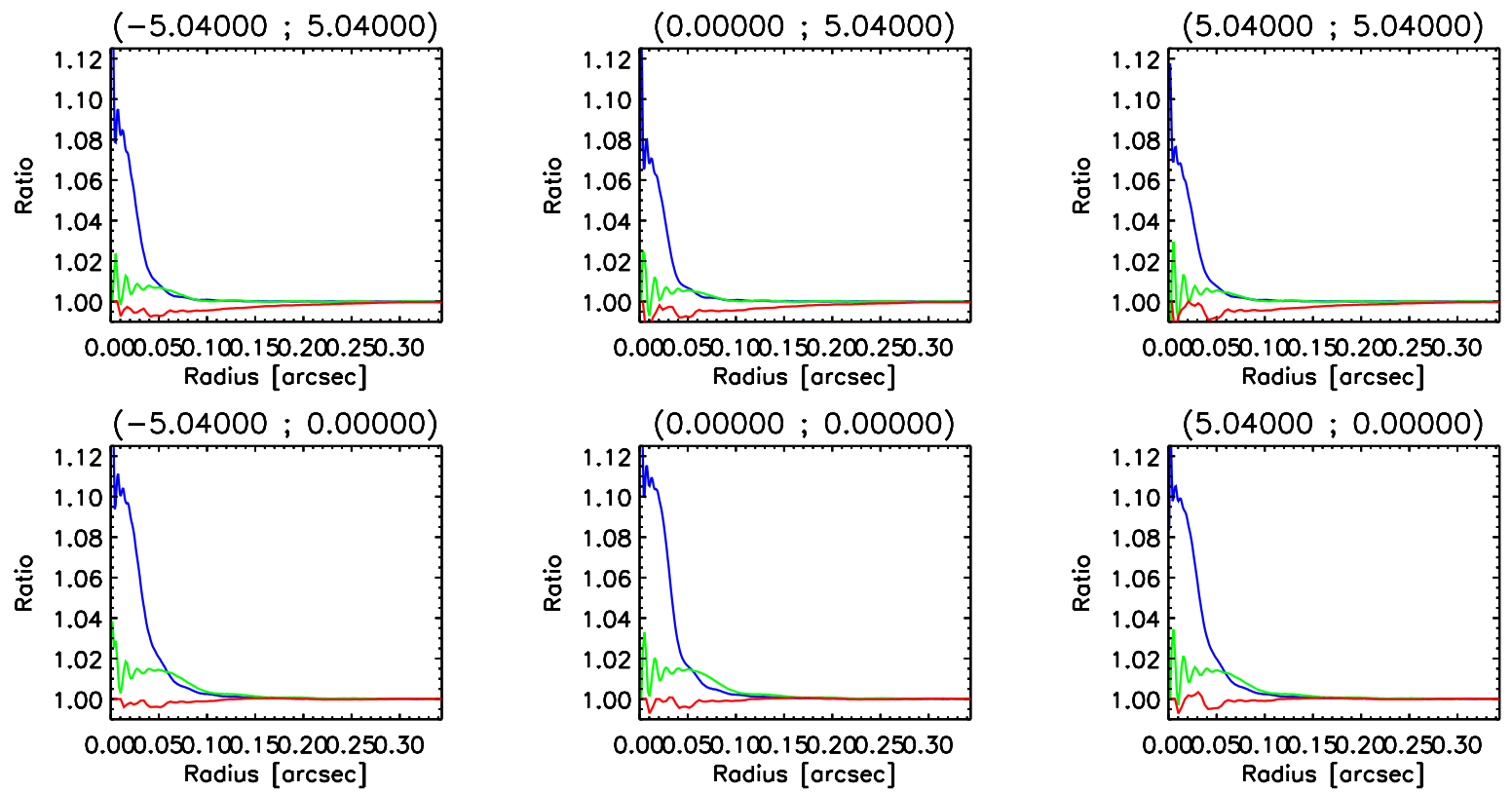

$(5.04000 ; 0.00000)$
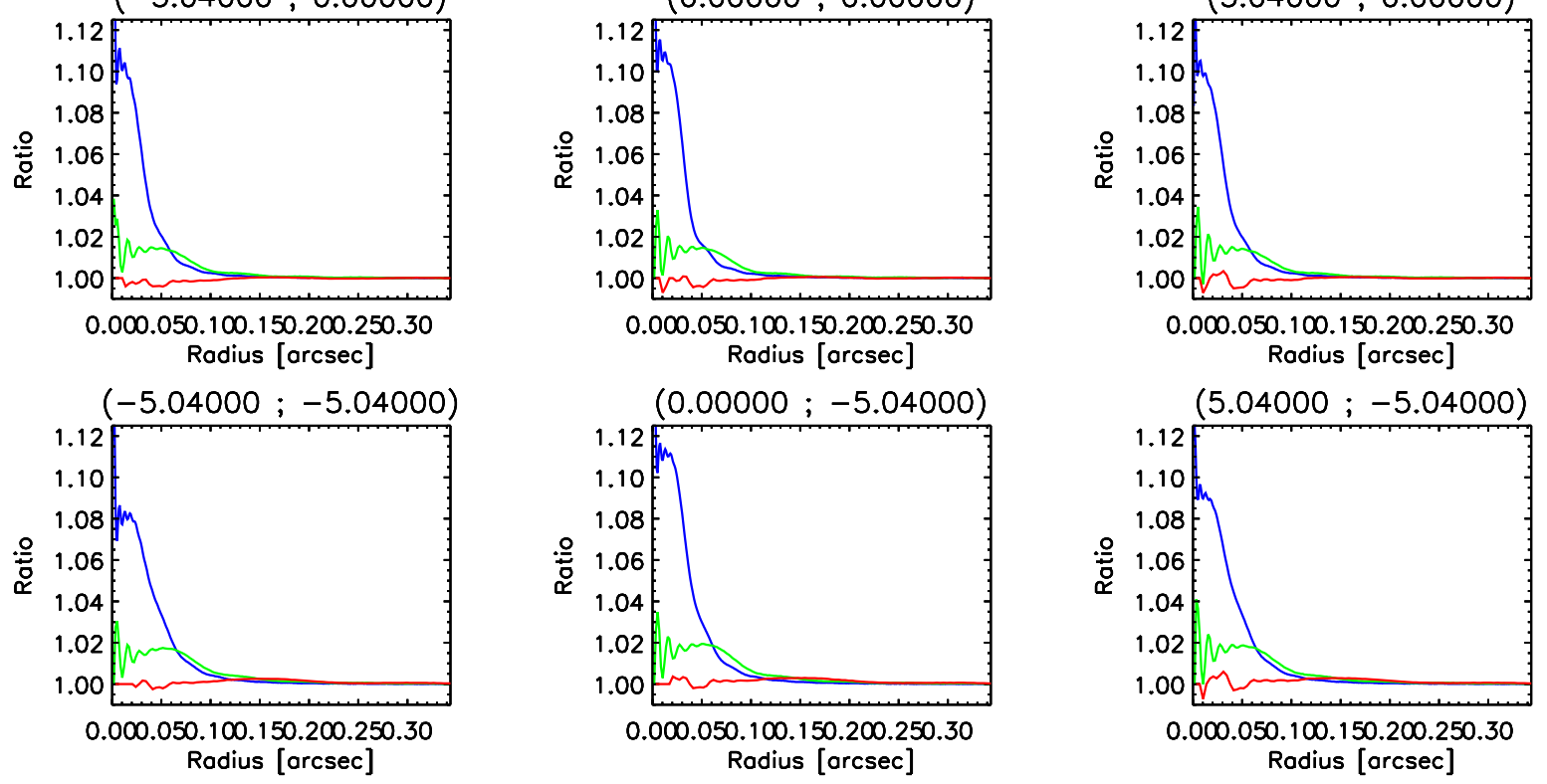

Figure 12.25.: Comparison of encircled energy fractions as in Fig. 12.24, but expressed as the ratio $\epsilon_{\text {NaGiCS }} / \epsilon_{\text {nocell }}$. Insertion of NAGICS slightly improves the encircled energy fraction.

reported in Fig. 12.28, in the same way as was presented for the nominal CRIRES in Fig. 12.14.

With NAGics, the marginal de-magnification is still in place, amounting to a range of displacements of -0.1 to $1 \%$ in $x$-direction (compared to $0-8 \%$ for normal CRIRES). The effect is thus slightly enhanced, but could be further compensated on the cost of FP image quality. In the $y$-direction, the displacement is actually better resembling the ideal position, with smaller deviations in the range -0.5 to $0.5 \%$ of the diameter (compared to $-1-8 \%$ for normal CRIRES). It is important to realize that the pupil image with NAGICS is not flipped (in $x$ and $y$ ) anymore, but rotated $90^{\circ} \mathrm{CCW}$. This is a result of the several foldings in the optical path. Likewise, the orientation of the image on the FP is also rotated $90^{\circ} \mathrm{CCW}$.

\subsubsection{Spectroscopic performance}

The motivation for the large OPL that NAGICs offers is the ability to produce deep and sharp absorption lines of suitable captive gases, preferably homogenously spread over a wide wavelength range (Chapter 10). To demonstrate the potential of the NAGICS OPL, I perform comparisons with captive gas calibrators that are already in use with CRIRES in a short-path calibration cell. 


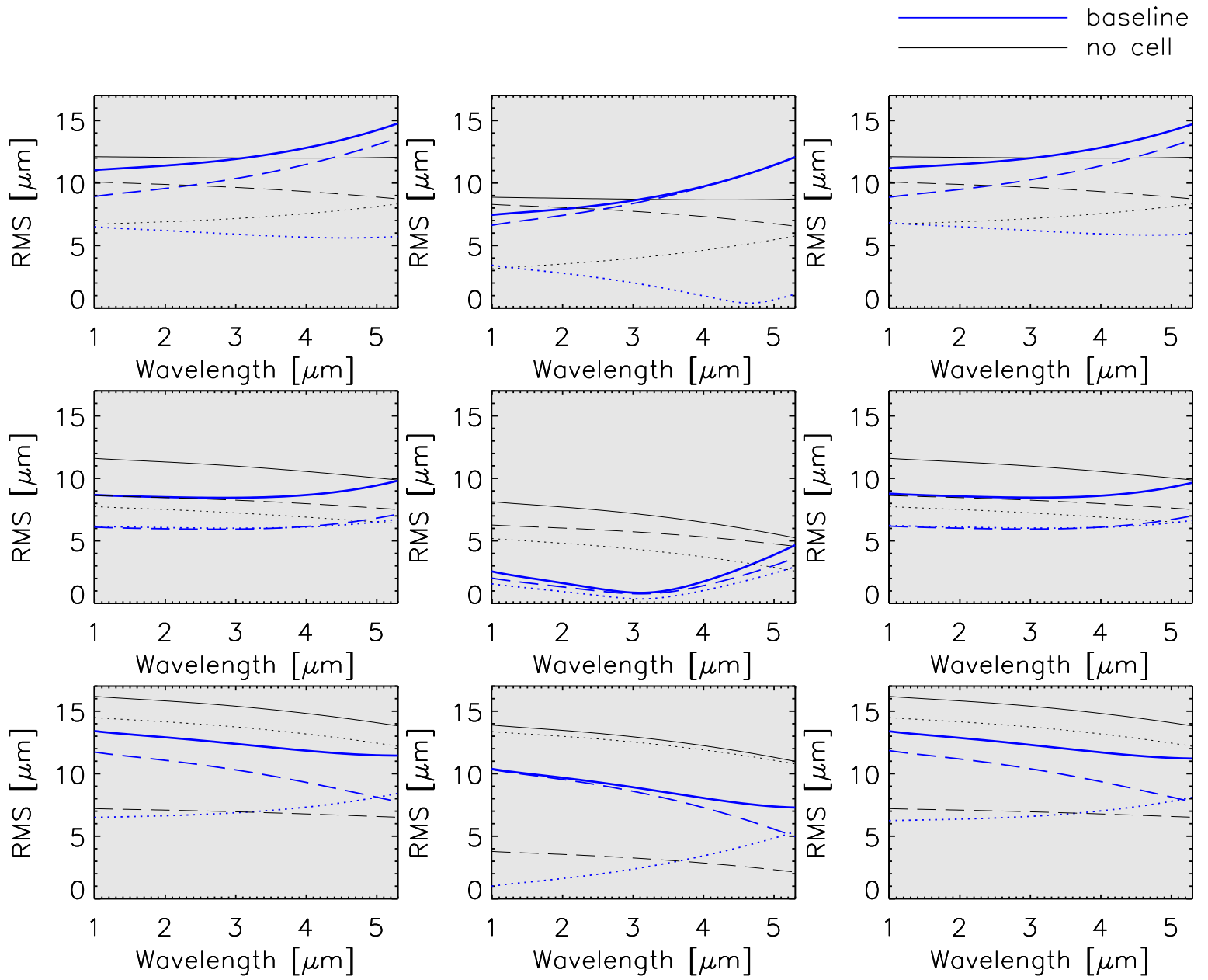

Figure 12.26.: RMS spot sizes in the FP for point sources imaged onto the slit. The spot sizes are determined for the system with NAGICS (blue lines) and without (black lines). To account for the spot distortions towards the edge of the $10^{\prime \prime} \times 10^{\prime \prime}$ field, the spot sizes are plotted as the radial RMS sizes (solid lines), and as the RMS sizes in X- (dotted lines) and Y-direction (dashed lines) separately. Each panel represents an off-axis field point (plus on-axis) across $10^{\prime \prime} \times 10^{\prime \prime}$, analogous to Fig. 12.24. See Fig. 12.27 for a visualization of the spot distortions. The NAGICS system outperforms the nominal image quality at the slit FP, and is diffraction limited at all wavelengths over almost the entire FoV.

Figure 12.27.: (on page 169:) NAGICS spot diagrams at the slit FP across a $10^{\prime \prime} \times 10^{\prime \prime}$ field, at the average focus position. Note that the box size shown for each field point is $100 \mu \mathrm{m}$ for $\lambda=0.95 \mu \mathrm{m}$ (blue, at top) and $2.3 \mu \mathrm{m}$ (green, middle), and $200 \mu \mathrm{m}$ for $5.3 \mu \mathrm{m}$ (red, at bottom). The system is diffraction limited in almost all cases across the $10^{\prime \prime} \times 10^{\prime \prime}$ field shown. 


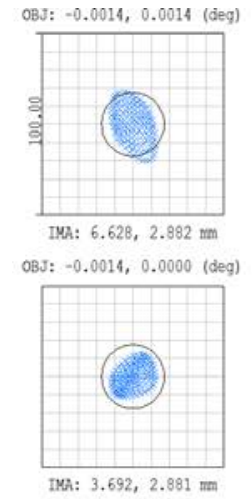

OBJ: $-0.0014,-0.0014$ (deg)

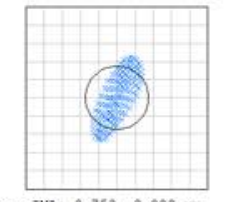

SLIT IF: $0.753,2.882 \mathrm{~mm}$

OBJ: $-0.0014,0.0014$ (deg)

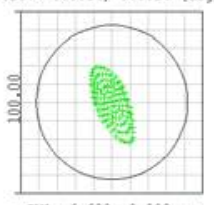

IMA: $6.638,2.882 \mathrm{~m}$

OeJ: $-0.0014,0.0000$ (deg)

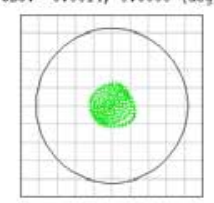

IMA: $3.701,2.881=$

OBJ: $-0.0014,-0.0014$ (deg)

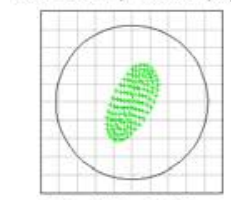

SLIT IF: $0.762,2.882=$

OBJ: $-0.0014,0.0014(\mathrm{deg})$

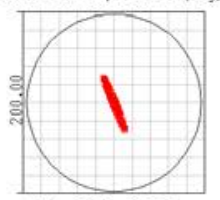

TKA: $6.675,2.883 \mathrm{~mm}$

OBJ: $-0.0014,0.0000$ (deg)

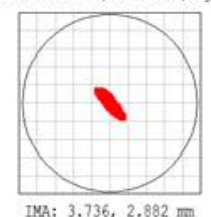

OBJ: $-0.0014,-0.0014$ (deg)

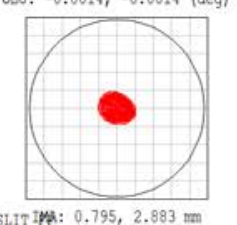

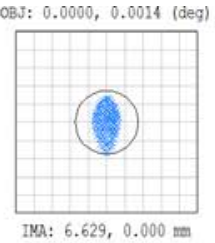

OBJ: $0.0000,0.0000$ (deg)

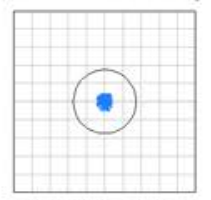

IMA: $3.693,-0.001=$

OBJ: $0.0000,-0.0014$ (deg)

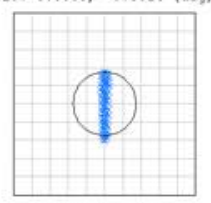

INA: $0.754,0.000 \mathrm{~m}$

OBJ: $0.0000,0.0014$ (deg)

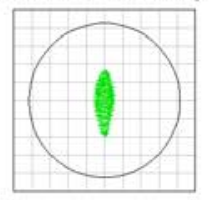

IMA: $6.639,0.000 \mathrm{~mm}$

OBJ: $0.0000,0.0000$ (deg)

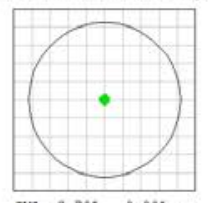

IMA: $3.701,-0.001=$

OBJ: $0.0000,-0.0014$ (deg)
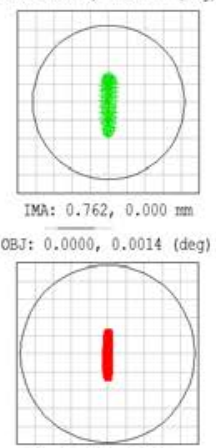

INA: $6.676,0.000 \mathrm{~m}$

OBJ: $0.0000,0.0000$ (deg)

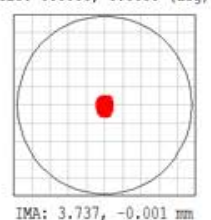

OBJ: $0.0000,-0.0014$ (deg)

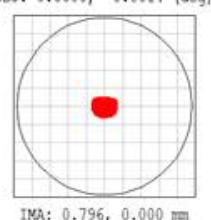

OBJ: $0.0014,0.0014$ (deg)

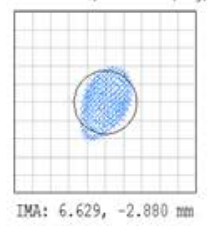

OBJ: $0.0014,0.0000$ (deg)

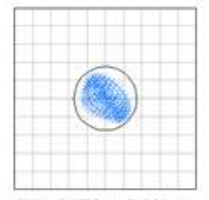

IMA: $3.692,-2.881 \mathrm{~mm}$

OBJ: $0.0014,-0.0014$ (deg)

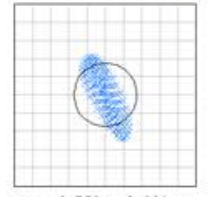

IMA: $0.753,-2.881 \mathrm{~mm}$

DJ: $0.0014,0.0014$ (deg)

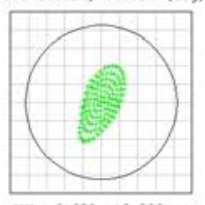

IMA: $6.638,-2.880=$

0QT: $0.0014,0.0000$ (deg)

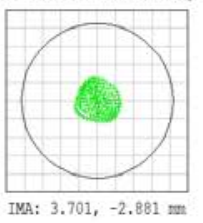

OBJ: $0.0014,-0.0014$ (deg)

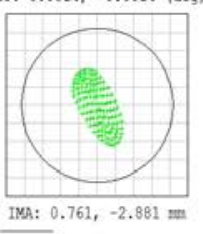

OBJ: $0.0014,0.0014(\mathrm{deg})$

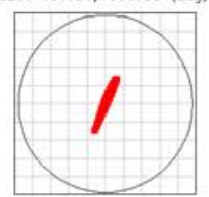

IXA: $6.676,-2.881 \mathrm{~mm}$

OBJ: $0.0014,0.0000$ (deg)

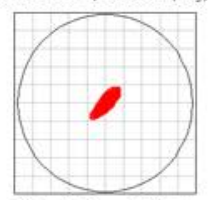

IMA: $3.736,-2.882 \mathrm{~mm}$

oss: $0.0014,-0.0014$ (deg)

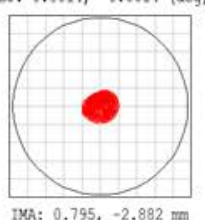




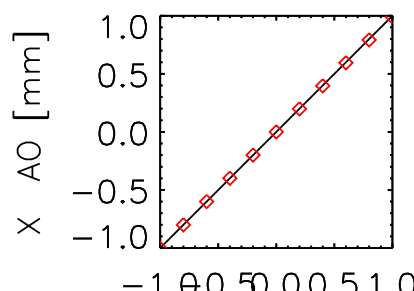

Rel. pupil coord. $X$

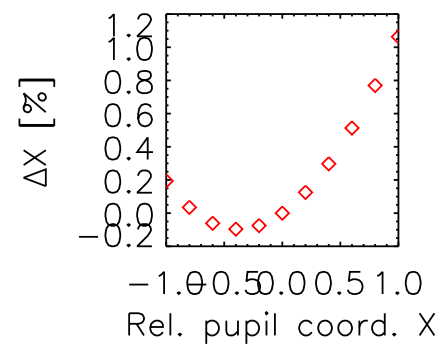

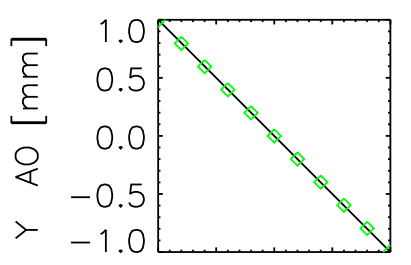

$-1 . \theta 0.50 .00 .51 .0$

Rel. pupil coord. Y

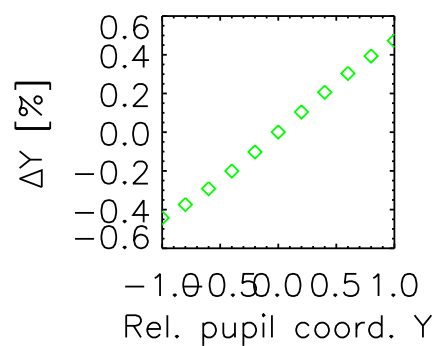

Figure 12.28.: Correspondence between the telescope pupil and the pupil image on the AODM with NAGICS. The position of imaged points on the AO-DM (in $\mathrm{mm}$ from centre, the diameter is $60 \mathrm{~mm}$ ) are determined as a function of the originating coordinates in the pupil plane (relative coordinates) in $X$ and $Y$ directions (left and right panels, respectively). The lower panels show the corresponding residuals. Note that for NAGICS, the pupil image is rotated $90^{\circ} \mathrm{CCW}$ with respect to the one of the nominal CRIRES. 


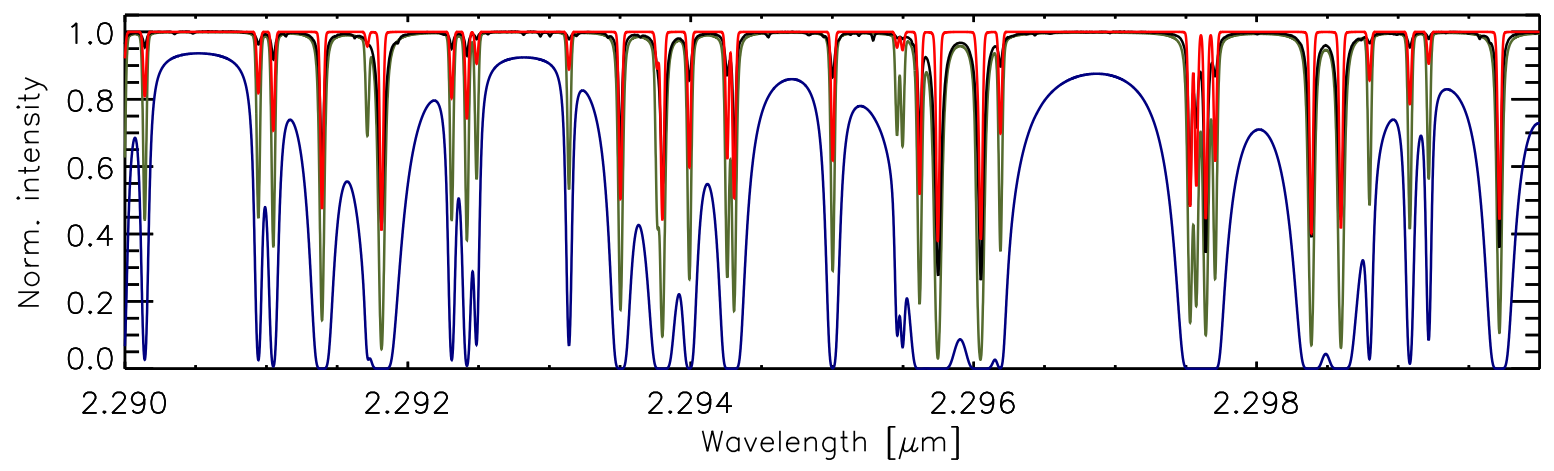

Figure 12.29.: Comparison of line widths and -depths for ammonia as the calibrator. An FTS measured spectrum of a $13 \mathrm{~cm} \mathrm{NH}_{3}$ gascell at 75 mbar pressure (black line; data courtesy Anglada-Escudé et al. 2012), and three synthesized spectra are shown: $\mathrm{NH}_{3}$ for NAGICS OPL and 75 mbar (grey line), 10 mbar (green line), and 1 mbar (red line), respectively. The latter are at $R=10^{5}$, while the FTS spectrum is at $R=7 \cdot 10^{5}$ (all at $20^{\circ} \mathrm{C}$ ).

\section{Ammonia}

Ammonia gas serves as a wavelength standard and has precisely characterized spectral transitions (for $\lambda>1.8 \mu \mathrm{m}$ ), with a wide spectral coverage in the NIR. For this purpose of a benchmark comparison, a transmission spectrum of ammonia $\left(\mathrm{NH}_{3}\right)$ is synthesized for the conditions provided by the NAGICS baseline design.

The spectral modelling is performed with the LBLRTM package (Clough et al. 1992, 2005), a branch of FASCODE, both of which are frequently used in climatology and athmospheric spectroscopy. LBLRTM is now in wider use for precision astronomical telluric modelling (Seifahrt et al. 2010). The spectroscopic line data is drawn from the HITRAN 2008 database (Rothman et al. 2005, 2009). For the baseline cell OPL $(\sim 2.96 \mathrm{~m})$, the ammonia absorption is computed for an ambient temperature of $20^{\circ} \mathrm{C}$ at pressures from 1 to 75 mbar. As a reference, an FTS-measured high-resolution spectrum of the ammonia cell constructed by Anglada-Escudé et al. (2012) is obtained. The data was kindly provided by G. AngladaEscudé, and was measured with $R=700000$ (at $2 \mu \mathrm{m}$ ) at $20^{\circ} \mathrm{C}$. Their cell has an OPL of $13 \mathrm{~cm}$ and is filled with pure $\mathrm{NH}_{3}$ at a pressure of $\sim 75 \mathrm{mbar}$ (also at the same temperature).

I show the recorded spectrum in Fig. 12.29 (black curve) in a small region of the $K$-band around $2.29 \mu \mathrm{m}$. This region is also used by Bean et al. (2010) with their short-path ammonia gascell and CRIRES (see Sec. 10.2.2). For comparison, a modelled spectrum of $\mathrm{NH}_{3}$ also with 75 mbar, but for NAGICS is included in Fig. 12.29 as the grey curve. The spectrum is degraded to match the resolving power of CRIRES $\left(R=10^{5}\right)$. At the given pressure, the ammonia lines are pressure broadened to a high degree, as a result of the $23 \times$ increased pathlength compared to the Anglada-Escudé et al. cell.

Line widths very similar to the FTS measurements $\left(R=7 \cdot 10^{5}, 13 \mathrm{~cm}\right)$ in this spectral region can be achieved with NAGICS at a pressure of only 10 mbar (shown in green), with considerably deeper lines. The potential of NAGICs becomes apparent when ultra-low pressures are applied. At 1 mbar (red curve), the line depths are mostly comparable to the Anglada-Escudé et al. cell at 75 mbar, but at this low pressure previously unresolved $\mathrm{NH}_{3}$ line blends and substructure becomes evident. Note that my 1 mbar spectrum is instrumentally broadened to $R=10^{5}$, while the $13 \mathrm{~cm}$ cell's spectrum (black curve) is at $R=7 \cdot 10^{5}$. 

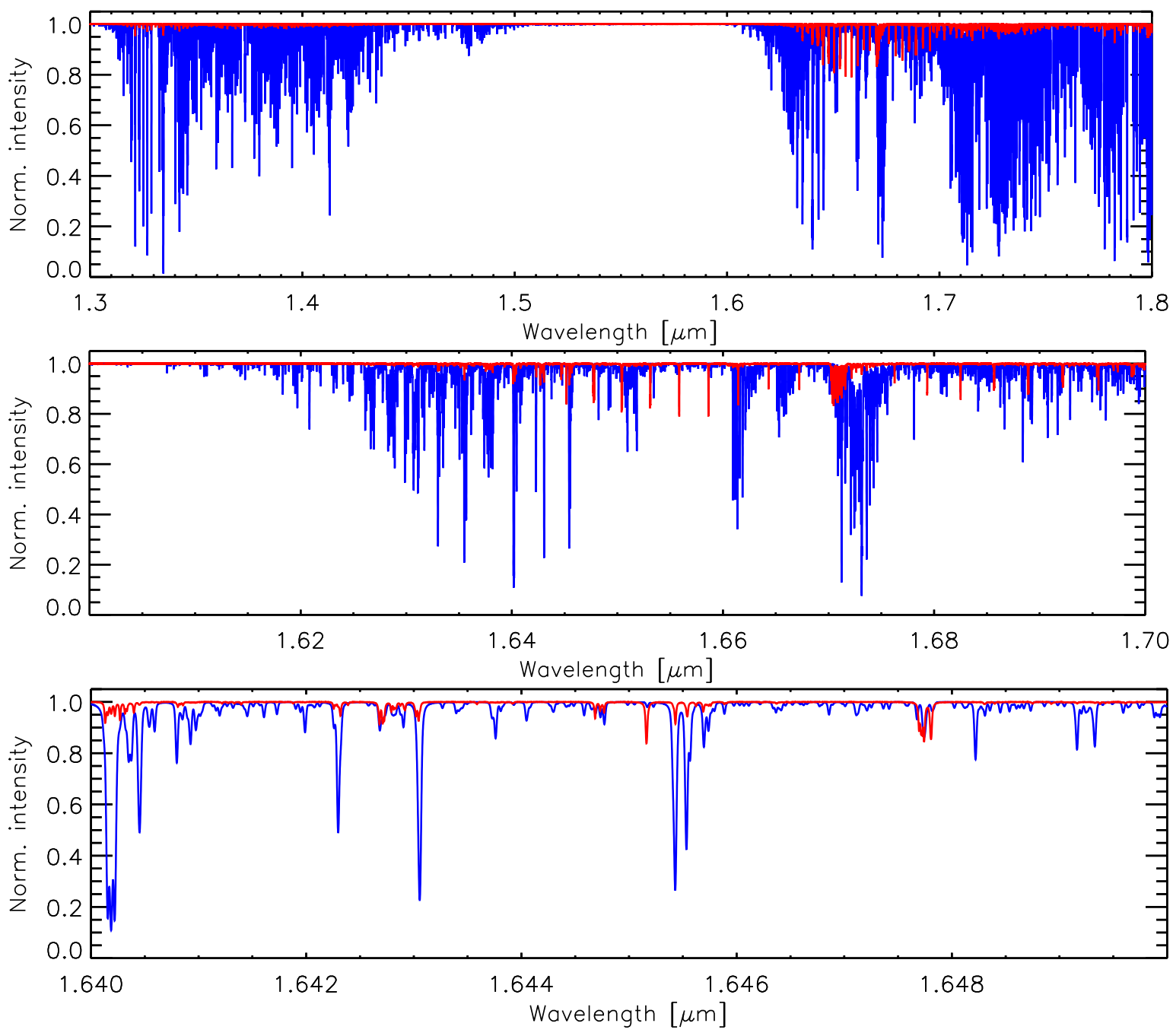

Figure 12.30.: Comparison of transmission spectra through the CsHeLL gascell and NAGICS. The captive gas is the methane isotopologue ${ }^{13} \mathrm{CH}_{4}$. The CsHELL-cell (Anglada-Escudé et al. 2012) is $13 \mathrm{~cm}$ long, at $\sim 275$ mbar pressure, and measured at $R=7 \cdot 10^{5}$ with an FTS (red curve). The NAGICS spectrum is synthesized for a $3 \mathrm{~m}$ OPL at $200 \mathrm{mbar}$, and for $R=10^{5}$ (blue curve). Panels (from top to bottom) show the $H$-band and two subset wavelength ranges.

The comparison makes clear that with the NAGICS OPL ultra-low gas-pressures are attainable that lead to increased line sharpness, while at the same time maintaining line depths. In the case of ammonia, the pressure thus can be tuned to attain the optimal balance between required line depth and perturbing line broadening. This ultimately increases the precision of the wavelength solution to higher levels than is achievable with current short-path ammonia gas cells.

\section{Methane isotopologues}

Methane is an abundant athmospheric greenhouse gas, and contributes significantly to the telluric absorption at NIR wavelengths. It is thus not convenient as a captive gas for astro- 
nomical calibration purposes, as the calibration lines would be superimposed on the same lines subject to unstable atmospheric perturbances. However, the isotopologue ${ }^{13} \mathrm{CH}_{4}$ only makes for a small natural abundance, and is thus a potential calibrator with a shifted spectrum that avoids confusion with the natural ${ }^{12} \mathrm{CH}_{4}$ (Anglada-Escudé et al. 2012).

To demonstrate the power of the NAGICS increased OPL, I explore the transmission spectra of ${ }^{13} \mathrm{CH}_{4}$ from recently measured FTS data, and from synthesized spectra for the properties of my baseline design. The FTS data was recorded at $R=7 \cdot 10^{5}$ through a $13 \mathrm{~cm}$ long gascell filled with ${ }^{13} \mathrm{CH}_{4}$ of $99 \%$ quoted purity, at $\sim 275 \mathrm{mbar}$ and $20^{\circ} \mathrm{C}$ (courtesy G. AngladaEscudé, priv. comm.). The spectrum is shown in Fig. 12.30 as the red curve for a wavelength range covering the $H$-band. Methane has very weak line bands for $\lambda \lesssim 2 \mu m$, so that the corresponding lines are very weak $(<20 \%$ depth) due to the small pathlength.

I compare this data to a synthesized spectrum of ${ }^{13} \mathrm{CH}_{4}$ for the $3 \mathrm{~m}$ OPL of NAGICS, with a pressure of 200 mbar. The spectral modelling is again performed with LBLRTM, with a linelist specifically extracted for the ${ }^{13} \mathrm{CH}_{4}$ isotopologue from the most recent HITRAN2012 database release ${ }^{7}$. This release features major additions for ${ }^{13} \mathrm{CH}_{4}$, increasing the wavelength coverage and the number of transitions by $250 \%$ compared to the previous HITRAN2008 version data for this methane isotopologue. The spectrum is plotted in Fig. 12.30 as the blue curve for the CRIRES resolving power of $R=10^{5}$.

Despite the lower pressure, the absorption coefficients of ${ }^{13} \mathrm{CH}_{4}$ in this wavelength regime result in drastically improved line depths compared to the short-cell. Although the line depths are strongly variable, the majority of the lines in the shown $H$-band exhibit depths of $60-80 \%$, with vastly increased coverage over the short-cell (cf. top panel in Fig. 12.30). NAGICS thus can open an opportunity for the simultaneous wavelength calibration in parts of the $\mathrm{H}$-band with ${ }^{13} \mathrm{CH}_{4}$, providing deep and relatively densely spaced lines. Note that the Anglada-Escudé et al. cell cannot be used for the $H$-band due to the very shallow lines, and that strong telluric absorption (mostly water-vapor) shortwards of $1.5 \mu \mathrm{m}$ and longwards of $1.8 \mu \mathrm{m}$ is superimposed on observations through the athmosphere. At my pathlength, ${ }^{13} \mathrm{CH}_{4}$ may be a viable constituent of a gas mixture for the wavelength range around $1.7 \mu \mathrm{m}$, complementing the much stronger line bands of methane (and its isotopologues) in the $K$ band and further red.

\subsubsection{Performance summary}

I have conducted a detailed performance analysis of the NAGICS baseline design, covering the optical design as well as application scenarios with two selected gas absorption species.

The optical baseline design meets all the requirements imposed by the CRIRES spectrograph (Sec. 11.2), and delivers diffraction limited image quality at the entrance slit. While the FoV is reduced compared to the nominal CRIRES FoV (matching the proposed slit length), the baseline design enables excellent Strehl ratios and encircled energy fractions.

The results show that in terms of image quality NAGICS outperforms the nominal CRIRES design at the instrument FP within this FoV, despite the introduction of a $3 \mathrm{~m}$ AOPL and 12 reflecting surfaces. The effiency loss of the spectrograph with NAGics can be kept as low as a factor of $<2$, which downshifts the CRIRES magnitude limit by about $\sim 0.5$ mag. My design is capable to retain the full CRIRES wavelength coverage.

A captive gas vessel that encloses the main parts of the NAGICs system has been identified as the more efficient solution over a nested vessel. Spectral modelling for the NAGICS filled with

\footnotetext{
${ }^{7}$ HITRAN2012 is accessible at http://www.cfa.harvard.edu/hitran/
} 
ammonia gas shows that lower pressures are attainable than currently in use, yet providing deeper and sharper calibration lines. A benchmarl test with the methane-13 isotopologue provides further evidence that suitable gas absorption features with deep lines can be achieved in domains that are not suitable with existing short-path calibration cells. 


\section{Risk assessment}

\section{Contents}

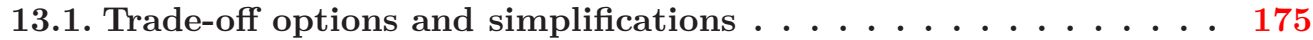

13.1.1. Field of view . . . . . . . . . . . . . . . . 175

13.1.2. Reducing optical complexities . . . . . . . . . . . . . . 176

RCRF off-axis parabolic mirrors . . . . . . . . . . 176

RCRF off-axis spherical mirrors . . . . . . . . . . . . . . 177

13.1.3. Wavelength range . . . . . . . . . . . . . . . . . 178

13.2. Tolerances . . . . . . . . . . . . . . . . . . . 181

13.2.1. Optomechanical alignment _. . . . . . . . . . . . 182

13.2.2. Tolerance analysis . . . . . . . . . . . . . . . 185

\subsection{Trade-off options and simplifications}

The NAGICs baseline model seeks to meet the requirements imposed by the VLt, CRIREs(+), the warm-optics, and by the calibration needs, while at the same time striving for a most flexible design approach. A number of free parameters in the design drive most of the complexity of the system. This concerns the manufacturing, assembly as well as integration, and lastly testing. Of primary concern is, eg. the type of curvature employed for the RCRF sub-system's mirrors, the desired FOV coverage, and the design strategy for the gas container vessel. These parameters affect all of the MAIT steps by rasing their complexity, and therefore pose specific risks on the realization of NAGICS.

A few selected aspects are discussed in the following, with the purpose of a trade-off between reduced system performance and risk. This is to estimate the impact of simplifications, reducing the overall risk, on the performance.

\subsubsection{Field of view}

Considerations on the limiting FoV have been discussed in Sec. 12.1 with respect to the (spatial) mounting and structure limitations. While a larger FoV can be realized with the NAGiCS concept at no fundamental design changes, larger (unvignetted) fields translate into larger optical elements, ie. surfaces (cf. Sec. 12.1.3). These increase the overall risk in several ways: first, a FoV exceeding $10^{\prime \prime} \times 10^{\prime \prime}$ is not compliant anymore with the baseline approach of mounting NAGICS on a two-story optical bench. Larger mounting stages, more clearance, and increased vertical spacing is required for mirror diameters exceeding $\sim 100 \mathrm{~mm}$ (baseline: $90 \mathrm{~mm}$ ). Second, the required mirrors at such apertures become increasingly risky (costly) to manufacture, specifically at the required custom curvatures. Third, testing these mirrors for their specifications is rendered increasingly demanding. A FoV larger than baselined serves mostly two reasons: the AO guidestar can be chosen with more flexibility, ie. farther away from the on-axis field. Also, the imaging capabilities are naturally expanded, facilitating simplified 
target acquisition, and allowing for a longer entrance slit (albeit its usability depends on the spectral format of the spectrograph).

On the other hand, further reducing the FoV is advantagous in terms of reducing system complexity, optical demands, and cost. However, the baselined $10^{\prime \prime} \times 10^{\prime \prime}$ FoV was motivated by the length of the anticipated CRIRES+ slit $\left(10^{\prime \prime}\right)$, fitted to the spectral echelle format (Follert et al. 2013). A reduced FoV down to the minimal $5^{\prime \prime} \times 5^{\prime \prime}$ (see Sec. 12.1.3) cannot fully exploit the spectral format, leading to reduced spatial resolving capabilities, and ultimately to a hampered sky background determination in the dispersed spectrum. The AO guidestar proximity is then also limited, as stated above.

For these reasons, a change of the design FoV is not deemed justified, unless limitations in mirror fabrication apply.

\subsubsection{Reducing optical complexities}

The NAGICs baseline design features only flat mirrors in the FO and DL sub-systems, and four conical off-axis mirrors in the RCRF sub-system. The four RCRF mirrors pose a substantial share of the total NAGICS risk, as fabrication, verification of the specs, and alignment of such mirrors is intrinsically more difficult than eg. spherical mirrors (see also Sec. 13.2).

To reduce the risk imposed by the MAIT of the RCRF mirrors, I explore options to employ mirror shapes with reduced complexity in the RCRF sub-system. Two possibilities are looked into: a) RCRF units with purely parabolic mirrors, and b) spherical mirrors. In either case, the off-axis application is retained.

\section{RCRF off-axis parabolic mirrors}

The NAGICS model is altered to encompass only parabolic off-axis mirrors as powered mirrors in the RCRF. The system thus comprises four degrees of freedom less, and a stable solution

Table 13.1.: Compliances with requirements for the NAGICS mirror trade-off process. Each trade-off option is listed with the corresponding combination of mirror selection, and whether the requirement on diffraction limited image quality in the slit FP is satisfied.

\begin{tabular}{|c|c|c|c|c|c|c|c|c|c|c|}
\hline \multicolumn{2}{|c|}{ FO } & \multicolumn{2}{|c|}{ DL } & \multicolumn{6}{|c|}{ "RCRF sub-system } & \multirow[t]{2}{*}{ DLim. $^{a} ?$} \\
\hline FM1 & FM8 & FM2 & FM3-5 & FM6 & RCM1 & RCM2 & RFM1 & RFM2 & FM7 & \\
\hline flat & flat & flat & flat & flat & free $^{b}$ & free & free & free & flat & $\checkmark$ \\
\hline flat & flat & flat & flat & flat & $\mathrm{pbl}^{c}$ & $\mathrm{pbl}$ & $\mathrm{pbl}$ & $\mathrm{pbl}$ & flat & $\pm \checkmark$ \\
\hline flat & flat & flat & flat & flat & $\operatorname{sph}^{d}$ & sph & sph & sph & flat & no \\
\hline flat & flat & sph & flat & flat & sph & sph & sph & sph & flat & no \\
\hline flat & flat & sph & flat & flat & sph & sph & sph & sph & sph & close \\
\hline flat & flat & sph & flat & sph & $\mathrm{sph}$ & $\mathrm{sph}$ & sph & sph & $\mathrm{sph}$ & close \\
\hline flat & flat & flat & flat & sph & sph & sph & sph & sph & sph & no \\
\hline flat & flat & sph & flat & sph & sph & sph & sph & sph & flat & no \\
\hline
\end{tabular}

Notes.

(a) Diffraction limited image quality in the FP.

(b) free: free conic. ${ }^{(c)}$ pbl: parabolic.

(d) sph: spherical. 
is seeked for. All other design parameters are kept unchanged. Emphasis is put on all requirements but image quality in the slit $\mathrm{FP}$, to allow for a comparison based on image quality, while the remaining aspects are fixed (ie. satisfied).

The model converges to a solution where the combination of powered mirrors and distances (including the delay-line) effectively compensates for the reduced degrees of freedom. I evaluate the system performance in the following in terms of achieved encircled energy fractions and RMS spot sizes.

The parabolic NAGICS configuration delivers almost diffraction limited image quality across the entire $10^{\prime \prime} \times 10^{\prime \prime}$ FoV. Fig. 13.1 plots the obtained RMS point image spot radii over wavelength, and Fig. 13.2 shows the measured encircled energies, also by field.

As in Fig. 12.26 for the baseline design, the $x$ and $y$ radii components are plotted separately in Fig. 13.1 as dotted and dashed lines, to indicate aspheric spot shapes. The loss of image quality compared to the baseline depends on the field, and also on the wavelength, and is most pronounced on-axis. On average, however, the parabolic configuration suffers from a degradation by a factor of 3-4 off-axis, and up to a factor of $\sim 15$ on-axis. That said, this amount of deterioration still enables a diffraction limited performance in most cases (within the greyed range in Fig. 13.1), ie. for $\lambda \gtrsim 2.3 \mu \mathrm{m}$.

In terms of encircled energy fractions (Fig. 13.2), a parabolic system cannot meet the expectations of the baseline design. The $80 \%$ level (on-axis) is achieved only within a PSF enlarged by a factor of $\times 3.5, \times 1.8$, and $\times 1.2$, for $\lambda=0.95,2.3$, and $5.3 \mu \mathrm{m}$. Hence, the at bluer wavelengths the efficiency of the spectrograph suffers the most, at the corresponding penalties. At off-axis, the losses are slightly smaller. Recall that the observing throughput depends crucially on the actual seeing conditions (and on the gain by an active AO), yet with a parabolic configuration of NAGICS, significant losses in throughput at the shortest wavelengths under seeing un-limited conditions are due. At longer wavelengths, the disadvantage vanishes.

\section{RCRF off-axis spherical mirrors}

Further reduction of complexity is achieved with spherical mirrors in the RCRF sub-system. No converging solution for such a NAGICS configuration can be found that yields performance damages of less than two orders of magnitude worsening, compared to the baseline design.

If more degrees of freedom for compensation are added, ie. in the form of converting flat folding mirrors at pronounced locations into spherical, powered mirrors, a drastic gain in optical performance can be attained. Table 13.1 lists the combinations of such conversions, and their benefit. The least complex option with the highest gain is comprised of six spherical mirrors, thereby converting two flat mirrors (FM2 and FM7). This combination allows for a model solution providing image quality in the range of the diffraction limit.

The performance of this six-fold spherical configuration is on display in Figs. 13.3 and 13.4 (RMS spot radii and encircled energy fractions). Such a system can deliver diffraction limited performance for $\sim 3 \mu \mathrm{m}$ on longwards (without active AO), but not much below (pre tolerancing!).

For the encircled energies, the penalty factors are about 5.2, 2.6, and 1.5 at the $80 \%$ level (on-axis, $0.95 ; 2.3$, and $5.3 \mu \mathrm{m}$, respectively). This means that with a slidwidth of $0.2^{\prime \prime}$ $\left(R=10^{5}\right)$, the nominal energy fraction entering the spectrograph is significantly less than $100 \%$ at all wavelengths, before seeing comes into play. Although the AO in closed loop may be able to boost this fraction to some extent (only under very good conditions), such a spherical configuration does not appear advisable as a NAGICS design for CRIRES. However, 

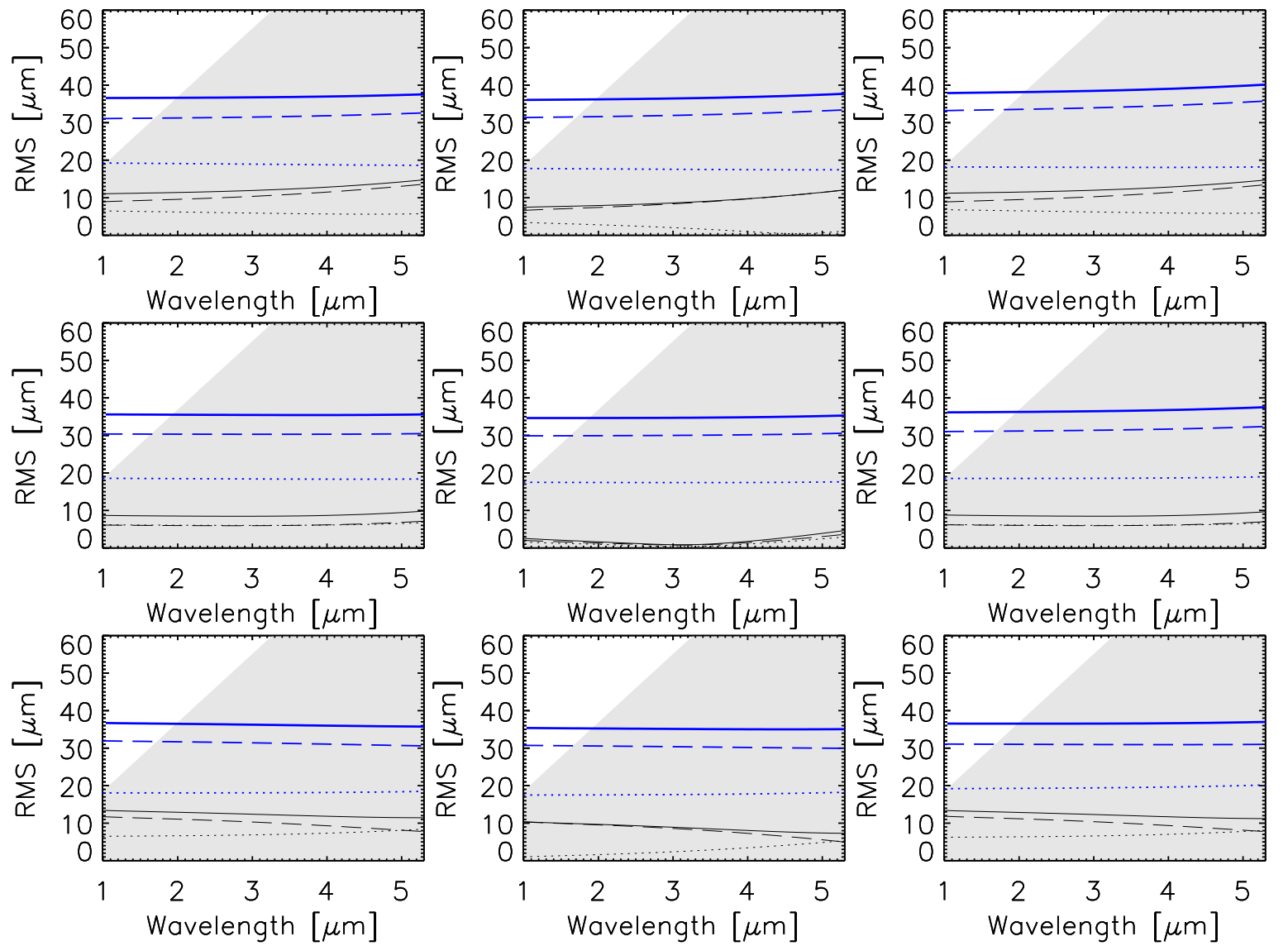

Figure 13.1.: RMS spot sizes for the trade-off option RCRF off-axis parabolic mirrors (blue), compared to the NAGICs baseline design (black). The panels represent the field point in the same way as in Fig. 12.26. The diffraction limited range (Airy radius) is indicated by the greyed area.

attributed to its reduced complexity, it may serve as a viable option for prototyping and a proof of concept study at reduced cost and risk.

The trade-off options are summarized in Table 13.1. While the parabolic setup may serve as a fall-back solution for NAGICS with reduced performance, the spherical configuration can be expected to suffer from high performance losses under real conditions. In effect, it depends on the capabilities of the $\mathrm{AO}$ whether it can significantly enhance the resulting perturbed wavefronts, and whether an $\mathrm{AO}$ correction is deemed mandatory, to specify the required NAGICS performance.

\subsubsection{Wavelength range}

The efficiency of the NAGICS system is limited primarily by the number of reflecting (coated) surfaces employed, and the number and type of windows used. For the large CRIRES range of wavelengths, the choice of materials with highest available reflectance or transmission, respectively, is very limited (see Sec. 12.3.1). 

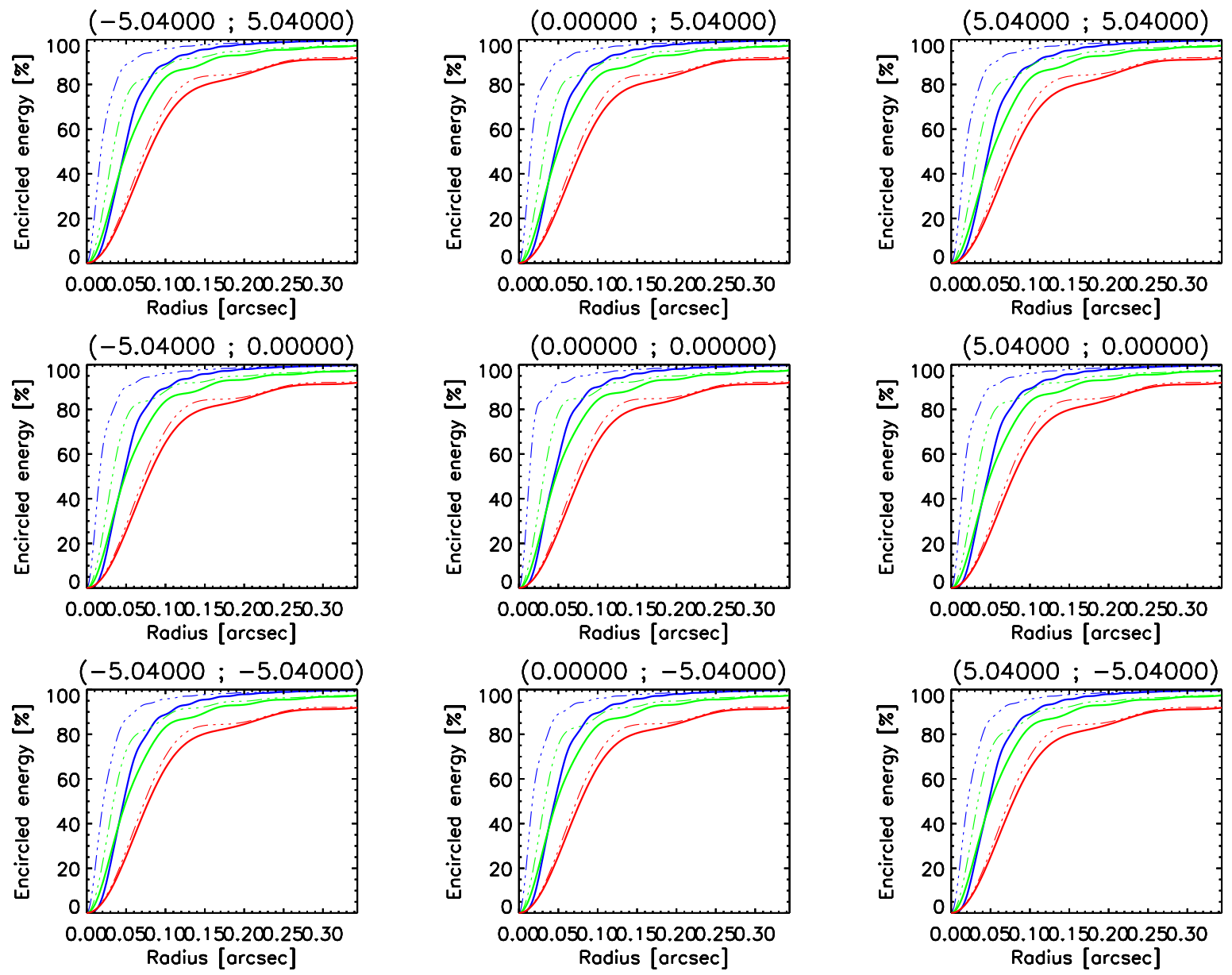

Figure 13.2.: Comparison of encircled energy fractions obtained at the slit for the parabolic trade-off option (solid lines), and the NAGics baseline design (dashed-dotted lines). Energy fractions are computed for the wavelengths $0.95 \mu \mathrm{m}, 2.3 \mu \mathrm{m}$, and $5.3 \mu \mathrm{m}$ (blue, green, red, respectively), for the nine field edges and the center. Other symbols as in Fig. 12.24.

The efficiency can be boosted, if the operating wavelength range is limited. In this case, window substrates with higher transmission rates as the baselined $\mathrm{CaF}_{2}$ can be envisioned. Two options are within reach: for a wavelength window restricted to $\sim 3.5<\lambda \leq 5.3 \mu \mathrm{m}$, $\mathrm{BaF}_{2}$ can be obtained with a transmission very close to $100 \%$, yet with below $90 \%$ outside of this range $(0.2-11 \mu \mathrm{m})$. The corresponding gain in efficiency is shown in Fig. 13.5 and is about a $5 \%$ improvement over the baselined solution (longwards of $3.5 \mu \mathrm{m}$ ).

The down-side of this trade comes two-fold. Besides the limitation on (efficiently) usable spectroscopy only in the $L$ and $M$ bands, the AO functionality is severely hampered, as the $R$ band suffers extensively from transmission losses. The material is also susceptible to humidity (degrading transmission), which should not pose a high risk in the Paranal environment, but may require careful handling and testing conditions. Limiting $\lambda$ in this way requires that a suitable calibration gas in that range be available to provide sufficient absorption lines. This itself poses a risk as the corresponding wavelength range is poorly studied with respect to astronomical wavelength calibration. 


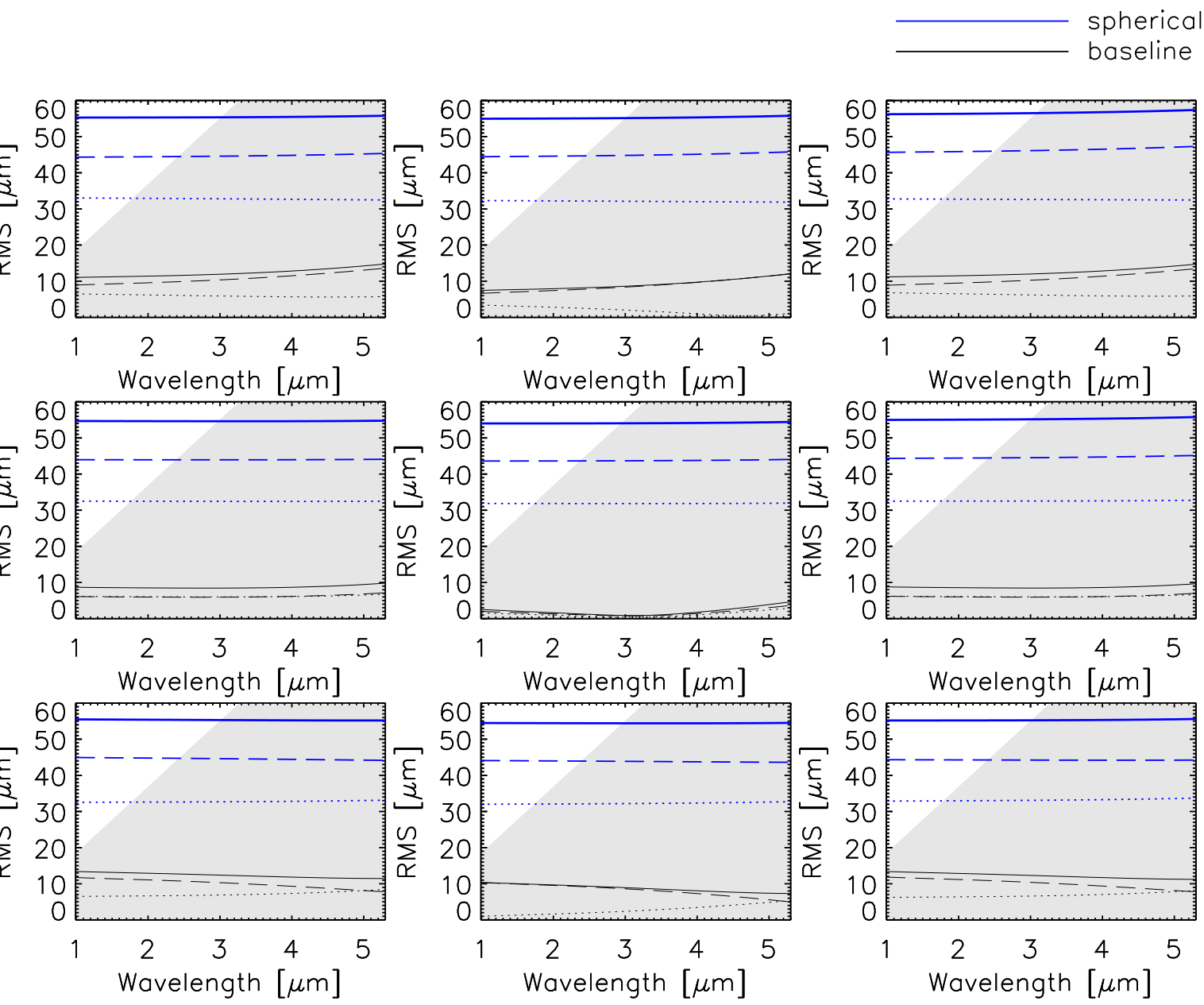

Figure 13.3.: RMS spot radii for the trade-off option RCRF off-axis spherical mirrors (blue), compared to the NAGICS baseline design (black). The panels represent the field point in the same way as in Fig. 12.26. The diffraction limited range (Airy radius) is indicated by the greyed area.

Other potential window substrates are UV fused silica (UVFS) or similar, more specialized types, which provide a $\gtrsim 94 \%$ transmission level over the $0.5<\lambda 2.0 \mu \mathrm{m}$ range. ${ }^{1}$ The spectroscopic coverage with CRIRES is thus restricted to shortwards the $K$ band (or including the $K$ band if a low-OH version is applied, which does not show the typical strong absorption bands around $1.4 \mu \mathrm{m}$ and $2.2 \mu \mathrm{m}$ ). The $\mathrm{AO}$ functionality is preserved to the extent of the baseline performance (with a few extra percent gain), and the effeciency is improved by $5 \%$ in the $K$ band with respect to the baseline design Fig. 13.5 shows the NAGICS efficiency with two $\mathrm{N}-\mathrm{PK} 52 \mathrm{~A}$ windows.

As concluded in Sec. 12.3.1, the limiting agent is the mirror coating. This means that any of the alternative window substrates will only play their strengths in terms of a substantial gain in system efficiency if there are more than just the two baselined windows employed (ie. a nested vessel configuration). In that case (six windows), $\mathrm{BaF}_{2}$ and eg. N-PK52A can make a

\footnotetext{
${ }^{1}$ High-transmission glasses such as the Schott type N-PK52A achieve $>99 \%$ internal transmission for $0.5<$ $\lambda<2.0 \mu \mathrm{m}$, but are also limited to $2 \mu \mathrm{m}$.
} 

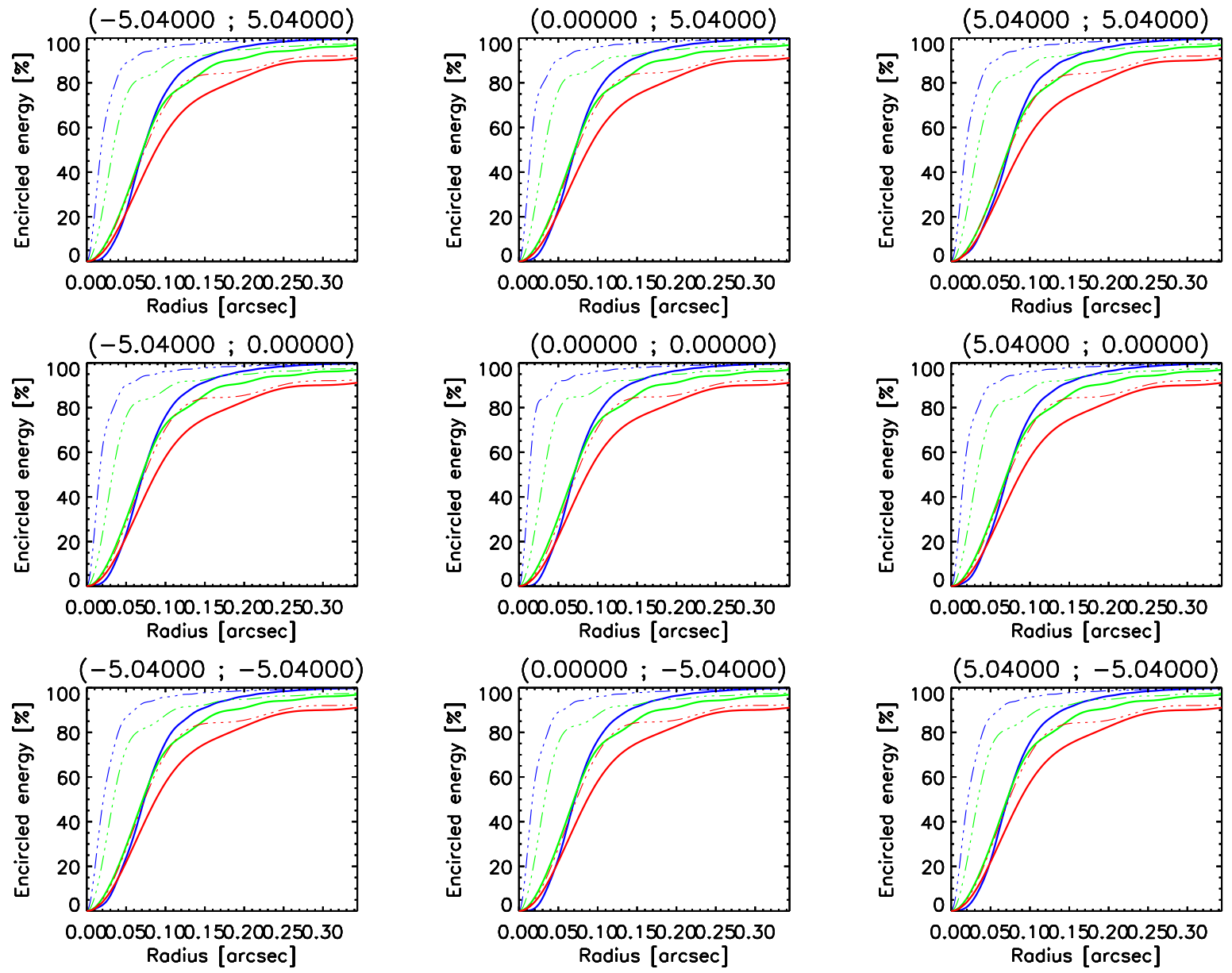

Figure 13.4.: Comparison of encircled energy fractions obtained at the slit for the spherical trade-off option (solid lines), and the NAGics baseline design (dashed-dotted lines). Energy fractions are computed for the wavelengths $0.95 \mu \mathrm{m}, 2.3 \mu \mathrm{m}$, and $5.3 \mu \mathrm{m}$ (blue, green, red, respectively), for the nine field edges and the center. Other symbols as in Fig. 12.24.

difference, with a resulting improvement of system transmission $\times 1.5$ and $\times 1.6$, respectively, over $\mathrm{CaF}_{2}$. Yet, a nested vessel comes at a transmission $\times 1.5$ worse than the baselined enclosure vessel.

Hence, the merits of alternative high-transmission window materials do not counterbalance the drawbacks of restricted wavelength coverage.

\subsection{Tolerances}

This section provides some estimates on the deterioration of the NAGICS baseline performance as a result of inevitable imperfections during the MAIT process. The NAGICS performance specifications as well as fabrication tolerances are yet to be defined, and are beyond the scope of this section. Instead, the estimates derived here on the resulting system performance shall a) help to constrain the allowable tolerances, mostly for manufacturing and alignment, and b) provide guidance on what performance can be expected under a reasonable set of assumptions 


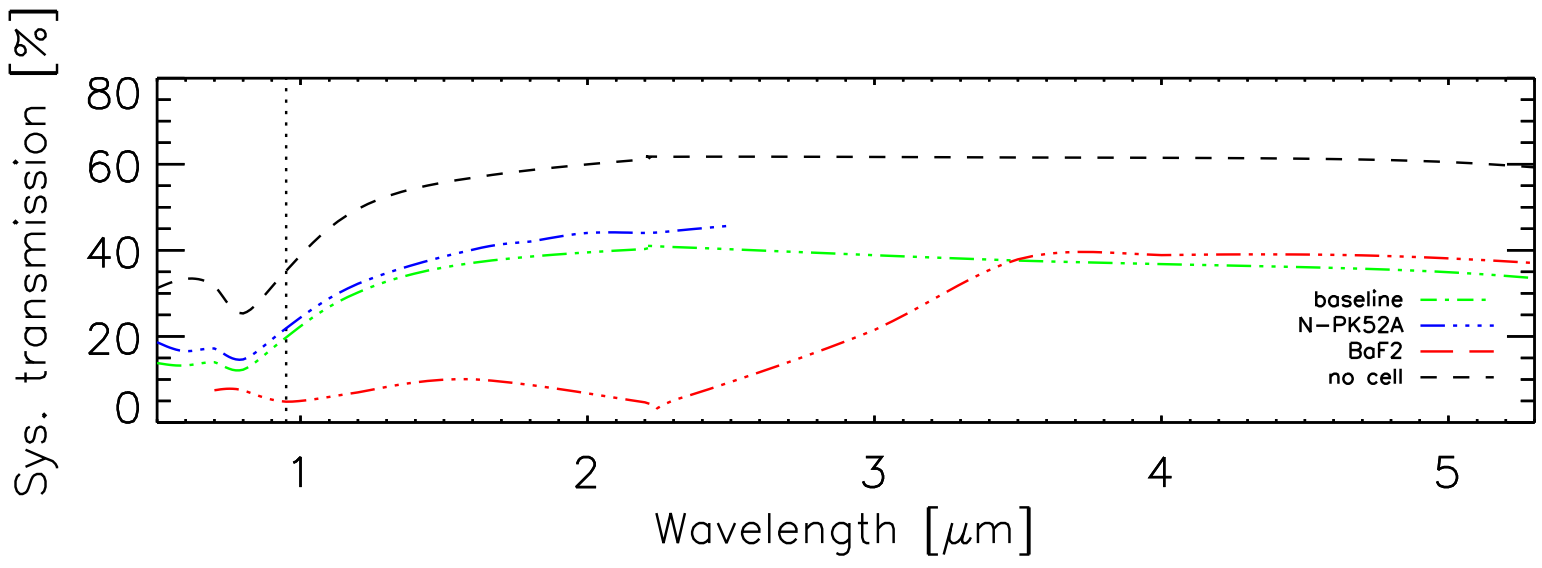

Figure 13.5.: Integrated system transmission as in Fig. 12.18 for the NAGICS baseline design $\left(\mathrm{CaF}_{2}\right.$ as window substrate), and two alternative materials with higher transmittance, but reduced wavelength coverage. Without NAGICS in the optical train, the "no cell" performance is achieved.

on tolerances.

\subsubsection{Optomechanical alignment}

The NAGics baseline design features 14 optically active surfaces, thereof eight flat folding mirrors, four powered (conic) mirrors, and two flat transmission windows. Although no specifications on manufacturing tolerances have been defined at this stage, the integration and alignment alone pose a challenge, and the resulting risk is on the optical performance. Even with ideally fabricated components, their precision alignment is a demanding process that crucially dictates if the anticipated system performance analyzed in Chapter 12.3 can be met, and to what level.

The 14 elements that NAGICS is comprised of will react differently on alignment errors, and the combination of errors for all components will give different results than those estimated from the isolated, single element errors alone. I consider five sources of error in the following estimates. These are

1. Offsets in distances (spacing) between subsequent components,

2. Centration errors in the plane perpendicular to the OPX (decenter in X and Y),

3. Erroneous tilts about the axes perpendicular to the OPX (X and Y tilt),

4. Deviations from the optimal radius of curvature for both the powered mirrors and the flat mirrors (flatness), and

5. Imperfections in the non-spherical shape of the powered mirrors (conic error).

The folding mirrors FM1-FM8 are the least subject to spacing errors (within reason) and decenters, while the RCRF mirrors can be expected to be very sensitive to variations in their conic, as they are operated highly off-axis. No assumptions are made on the surface quality (roughness) at this stage, and tolerances are assigned only to the NAGICS elements. For the telescope and remaining CRIRES warm-optics, the nominal design data is assumed. 

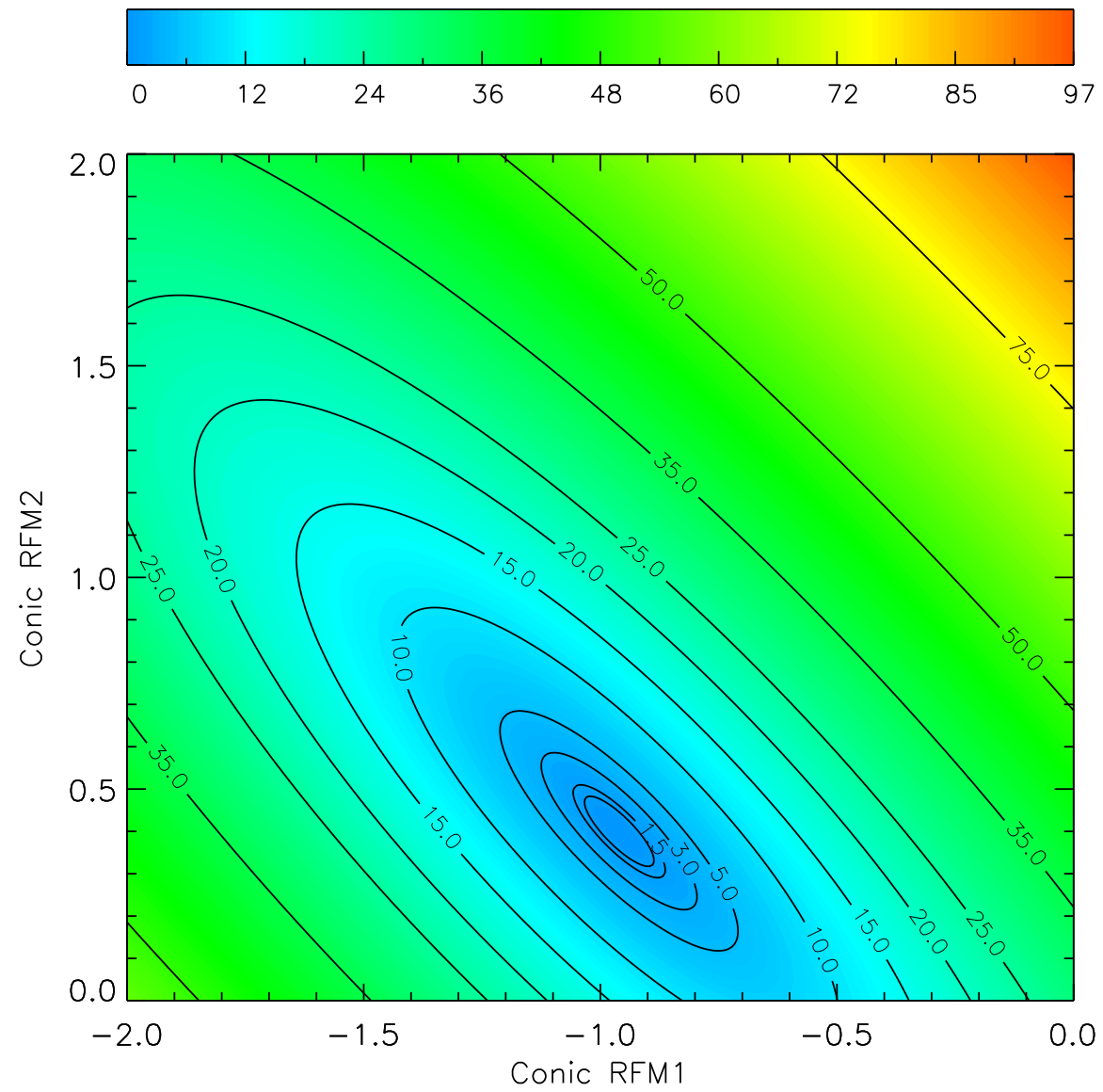

Figure 13.6.: Sensitivity map of variations in the conic constants of the RFM1 and RFM2 mirrors. The contours give the response of the system relative to the best values for RFM1 and RFM2.

For the five parameters above, the following conservative error budget is assigned. Spacings and decenters be accurate within $\pm 0.1 \mathrm{~mm}$, tilts $\pm 0.1^{\circ}$, deviations in RoC be within $\pm 0.1 \mathrm{~mm}$, and \pm 0.1 for the maximum conic error. Diameters are not considered, as these are sufficiently oversized. For each of the two transmission windows, the tilt of one side is limited to $\pm 1^{\prime}$ at a time (window wedge). This corresponds to a a typical value of parallelism for the window substrates. However, each window as a whole may have a tilt of up to $\pm 0.1^{\circ}$, independently of each other. This seems reasonable as the gas container vessel (with attached windows) does not need to be moved during normal operations (recall that only the FO sub-system is moved into and out of the Nasmyth beam).

The NAGics baseline model is iterated for each of the 14 surfaces, by measuring the effect of the five parameters with their corresponding errors. Each parameter is subsequently perturbed to the minimum and maximum of its error budget, and the system evaluated. The performance criterion monitored is the RMS radial spot size or wavefront error of the image in the FP (on the CRIRES+ slit), for a wavelength of $1 \mu \mathrm{m}$ (ie. about the blue edge of the CRIRES wavelength range). After each step, the system is allowed to adjust the focus position by re-focusing with the telescope M2 mirror to account for offsets in the FP location (the resulting offsets of the $\mathrm{FP}$ along the OPX are within a few $\mathrm{mm}$, the range to re-focus in the FP is $50 \mathrm{~mm}$ ). The analysis is carried out separately for the on-axis position and eight edge 


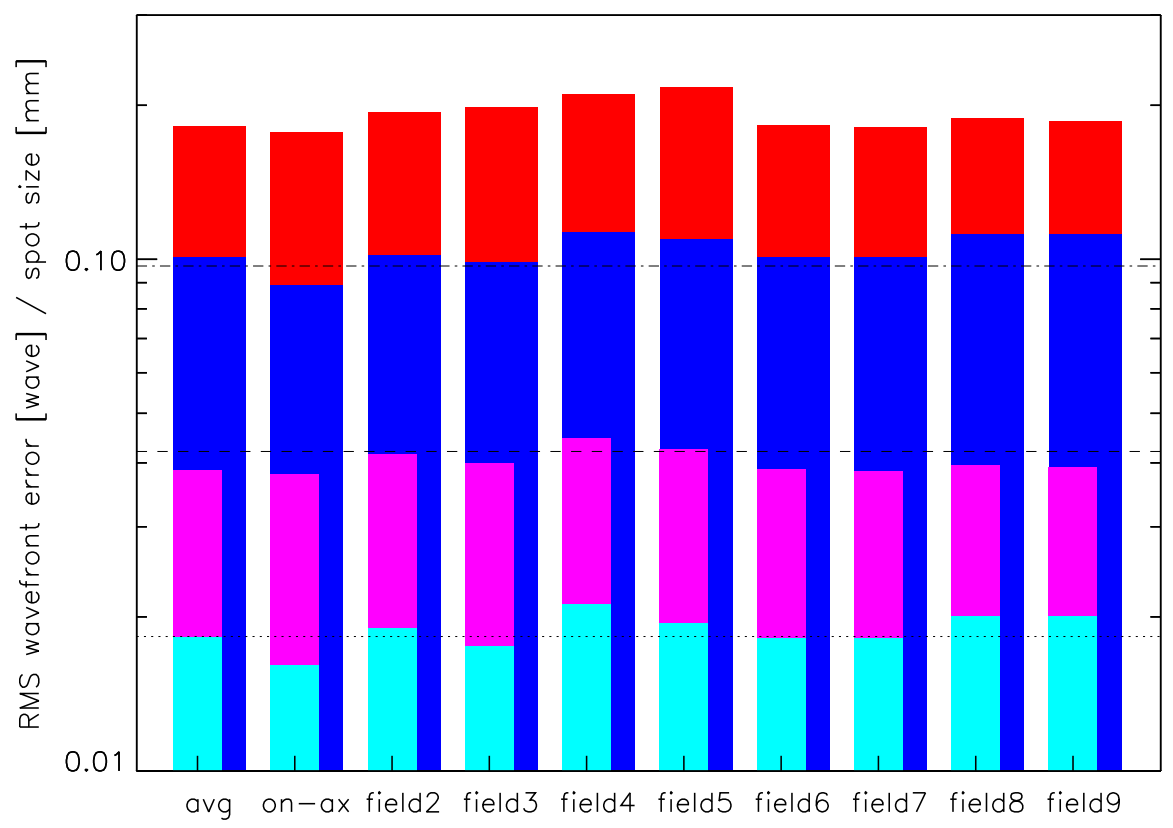

Figure 13.7.: (RSS-)estimates on the change of performance at $1 \mu \mathrm{m}$ reflecting the assumed tolerance budget for all parameters and surfaces. Performance is expressed in RMS wavefront error (broad bars, in waves) and RMS spot sizes (thin bars, in $\mathrm{mm}$ ). The blue and cyan bars give the nominal NAGics baseline performance in wavefront error and spot sizes, respectively. The red and pink bars indicate the root sum squared degraded total performances for the assigned error budgets. The first column is the average over the entire field, with the subsequent columns giving the individual field positions across the FoV. Horizontal lines mark diffraction limits (in $\mathrm{mm}$ ) for $\lambda=0.95,2.3$, and $5.3 \mu \mathrm{m}$, respectively.

and corner field angles in the FoV.

The results indicate that the by far strongest impact on the spot size or wavefront error is caused by errors in the RCRF mirror shapes, ie. the conic constants of the RCM2 and RFM1 units. The respective margins of the RCM1 and RFM2 units have a smaller impact by about a factor of four. The contribution of the possible wedge of the first transmission window, as a source of potential chromatism, is almost an order of magnitutde less than the impact of the RFM1. A sensitivity map of the conic variation for RFM1 and RFM2 is given in Fig. 13.6 as an example. The contours reveal a strong gradient towards a deficient performance (given in units of relative performance change compared to the best fitting values).

For the baseline design, a nominal wavefront error of 0.089 waves at $1 \mu \mathrm{m}$ is obtained for the on-axis position, and 0.101 waves averaged over the entire field. Following the root sum squared (RSS) method (Smith 1991), a combined resulting wavefront error can be estimated that reflects the tolerances of all parameters for all 14 surfaces (Youngworth 2006), given the margins above. Doing so reveals a total wavefront error of 0.177 and 0.182 waves respectively. These estimates indicate that a) the NAGICS design can compensate reasonable precision alignment errors to a large degree, owing to its highly symmetric concept. Based on these estimates, b) the degraded system performance can be expected to be still within a factor of three of the diffraction limit ${ }^{2}$. The results are summarized in Fig. 13.7 by field angle in terms

\footnotetext{
${ }^{2}$ A wavefront error of $\sim 0.05-0.07 \lambda$ is considered diffraction limited; Mahajan 1998.
} 
of RMS spot size and wavefront errors. for wavelengths

\subsubsection{Tolerance analysis}

In an independent approach, the NAGICS baseline behaviour to tolerances in the parameters described in the previous section is analyzed in a statistical approach. Monte Carlo simulations are performed for the performance indicators RMS wavefront error, and RMS spot size in the FP. The error budget per parameter is the same (see above). For each surface, the parameter values are randomly drawn from a normal distribution within $2 \sigma$ of the error margin assigned to that parameter. This means the normal distribution's mean is set to the mean of parameter value plus tolerance, and one standard deviation is a quarter of the tolerance range. In this way, the drawn parameter values are limited to the specified tolerances, and do not exceed them. The simulation is repeated $10^{5}$ times for each indicator, and the reaction of the system is measured in the corresponding indicator.

This procedure allows for a statistically meaningful population of all toleranced parameters at the same time, and reflects the probability distribution of perturbations in a true physical system.

Fig. 13.8 shows the results of the MC runs. The MC simulations are carried out independently for RMS wavefront error in the FP, and for RMS spot radius in the FP as a figure of merit (upper and lower panels, respectively). First, in the on-axis case the distributions (right panels) are shifted slightly to better performance (smaller wavefront error and spot radius), as is expected. The shift amounts to 0.01 waves or $\sim 2 \mu m$ in the maximum peak, and is thus small compared to the absolute errors, so that the average field seems to give a good description for the entire FoV including the sweet-spot on-axis.

Fig. 13.8 suggests that there is a high probability to yield a system with small resulting error, and a negligible likelihood to obtain very large errors, given the underlying alignment and fabrication errors assigned in Sec. 13.2.1. More quantitatively, the following picture arises: The most probable erroneous system with $\sim 6 \%$ of all cases has a wavefront error of 0.096 waves, or a resulting spot radius of $17.4 \mu \mathrm{m}$. This is a performance just below the diffraction limited level $(18.3 \mu \mathrm{m}$ spot) at $1 \mu \mathrm{m}$. Inspecting the cumulative probability function in Fig. 13.8 reveals that the probability to yield the system with diffraction limited performance at $1 \mu \mathrm{m}$ (RMS spot below $18 \mu \mathrm{m}$ ) is actually about $10 \%$. Within $1 \sigma$, the performance is degraded to less than a level of 0.130 waves or $25.9 \mu \mathrm{m}$. On the degraded end, the probability to end up with eg. a performance worse than 0.173 waves RMS wavefront error or $36 \mu \mathrm{m}$ spot radius is only $10 \%$. For comparison, the tolerance-free design at $1 \mu \mathrm{m}$ has a nominal error of 0.101 waves or $18.2 \mu \mathrm{m}$.

There are two implications of these figures. First, by assuming a set of low-precision tolerances for the NAGICS surfaces, the predicted resulting performance in terms of image quality in the FP is still on the order of the diffraction limited performance, with a $1 \sigma$ confidence. Second, by tightening the tolerances to a higher level of precision, a diffraction limited performance is feasible for NAGICS. The level of precision needed is subject to further analysis and depends on the specifications. However, from this concept analysis already it can be concluded that diffraction limited performance is can be achieved with modest demands on precision. 

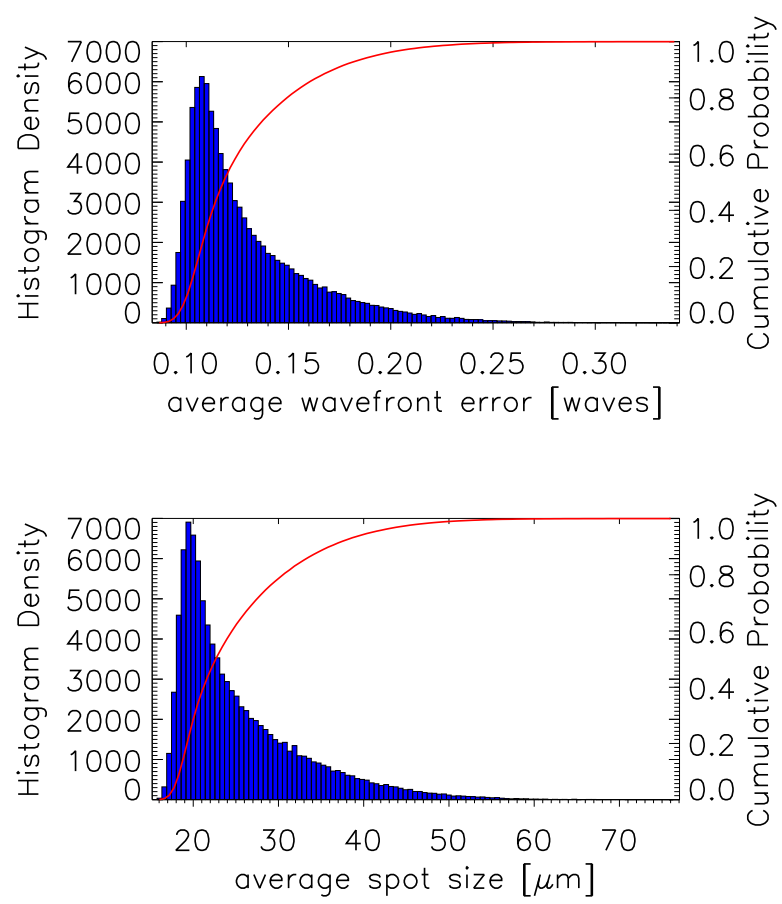
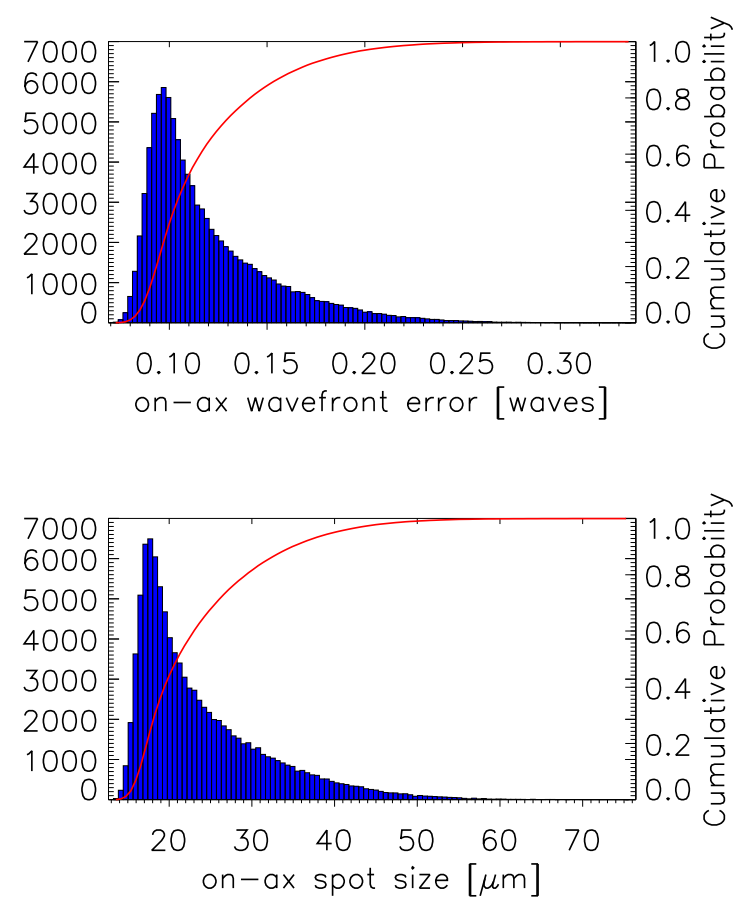

Figure 13.8.: Results obtained from $10^{5}$ Monte Carlo simulations, perturbing all toleranced parameters at the same time. The statistical performance result is expressed in RMS wavefront error (top panels) and in RMS spot size (bottom panels) at $1 \mu \mathrm{m}$. The statistics are only marginally different for the averaged field (left panels) and the on-axis position (right panels). For the averaged field, the most likely system settles with a wavefront error (spot radius) of $0.096(17.4 \mu \mathrm{m})$, with a probability of $5.9 \%(6.5 \%)$, respectively. With a $1 \sigma$ probability the system will not be degraded to worse than 0.130 waves $(25.9 \mu \mathrm{m})$, respectively, which is close to the diffraction limit $(18.3 \mu \mathrm{m}$ for $\lambda=1 \mu \mathrm{m})$. 


\title{
14. Summary and outlook
}

\author{
Contents
}

14.1. Adaptions to other instruments . . . . . . . . . . 187

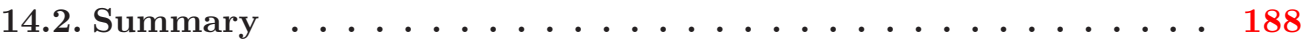

\subsection{Adaptions to other instruments}

The NAGICS baseline design (Sec. 12.1) is specifically tailored and optimized to the properties and requirements of CRIRES at the VLT. However, the NAGICS concept is a flexible approach that allows for scalability, most notably for the AOPL parameter. The system can thus be easily adapted to the needs of other instruments, and to other telescopes.

In this way, the NAGICS concept may be a viable option to equip other high-resolution optical or NIR spectrographs with an adaptable long-path calibration gascell. This approach is most useful with telescope/instrument setups where the space constraints along the optical path between telescope and instrument are tight, and do not allow for placement of a conventional long(-tube) gascell. In such cases, given that a short-path gascell cannot satisfy the needs, the provision of an AOPL of several meters by NAGICs is beneficial for several reasons: First, it enables to relax the required gas pressure needed to achieve the necessary line depths, while suppressing pressure broadening at the same time. Second, it enables the application of gases with low absorption coefficients, which over small pathlengths (at a given pressure) cannot provide enough absorption to form a strong transmission spectrum. Third, in mixtures of gases NAGICS provides the AOPL as an additional parameter to tune the selection of species and their partial pressures to find the optimal compromise for the desired wavelength coverage, line density, -depth and -sharpness.

For any instrument, NAGICS needs to be adapted and optimized to fit. The primary telescope parameters that will change the NAGICS properties are the focal ratio offered and the FoV accepted by the instrument (cf. Sec. 12.1.3). As with the CRIRES adaption of NAGICS, the resulting sizes of the main mirror units for larger AOPL are what constrains the feasibility most, and is subject to opto-mechanical limitations.

For long pathlengths, the system becomes advantagous if an intermediate focus is provided between the RCM2 and RFM1 units (cf. Fig. 11.19), instead of the beam being most collimated. Such a setup also eases to place conventional, tube-like cells with reasonable lenght between these two mirror units. This comes at the advantage of replacing such a cell vessel without the need to re-align, so that different captive gas-mixtures can be inserted on demand for a desired wavelength calibration. The longer the NAGICS length, the more feasible is also the implementation of a nested vessel type, inserted in between the whole RCRF subsystem (Sec. 11.3.5).

Test adaptations to the optical design of the New Technology Telescope (ESO/NTT) with a $3.5 \mathrm{~m}$ aperture and $\mathrm{f} / 11$ at its Nasmyth port (Thle 2012) prove diffraction limited performance for resulting pathlengths of $\sim 0.8 \mathrm{~m}$ up to $32 \mathrm{~m}$. 
For the E-ELT baseline design ${ }^{1}$, (f/17.7 at Nasmyth) the NAGICS concept likewise delivers diffraction limited performance. For the E-ELT case, constraining the FoV is a critical step for the reasons outlined above (the $10^{\prime} \mathrm{FoV}$ results in a $\sim 2.2 \mathrm{~m}$ FP diameter). In the latter case, additional flexibility is gained by the implementation of the AO. As the AO correction is part of the telescope, implemented with the M4, the requirement to pupil re-image as with CRIRES is relaxed.

The NAGICS concept can thus be adapted to a wide range of focal ratioes, and the examples given show that the optical design permits usable pathlengths of up to $32 \mathrm{~m}$ for gas absorption apllications.

\subsection{Summary}

Part II of this thesis presented a novel approach to a long-path gas absorption cell device for astronomical spectrographs. For the first time, a transmission calibration mechanism for simultaneous and offline wavelength reference measurements is conceived which enables optical pathlengths through a captive gas of several meters. This unprecedented capability is offered for an extended field of view, and is not limited to an on-axis point source as existing laboratory installments. The optical design features a compact format and allows insertion into and removal from the optical path. In both cases, the optical setup for a given telescope/spectrograph system does not need to be changed. To the spectrograph fed by the novel cell concept, the optical beam properties as without cell are restored.

For CRIRES at the Vlt I have developed this novel concept in detail to meet the CRIRES requirements and properties ("NAGICS"). This baseline design has been accurately modelled and its optical performance analyzed. The results demonstrate that NAGICS delivers diffraction limited image quality over the entire CRIRES supported wavelength range to the spectrograph, with virtually no degradation of performance over the full field of view required to illuminate the entrance slit. The analysis further shows that a high level of throughput is attainable with a purely reflective design ${ }^{2}$, while at the same time fullfilling all requirements (including adaptive optics functionality).

The gain in wavelength calibration fidelity has been estimated by modelling the transmission spectra of two molecular species for the NAGICS setup. The results for ammonia and the methane-13 isotopologue demonstrate that deeper and sharper absorption lines at lower pressures, with higher line densities, can be expected with a NAGICS gascell.

I have further discussed options to limit the supported wavelength range, and to reduce the optical complexity. Finally, an analysis for moderate optical alignment errors is presented. The results show that diffraction limited performance of a manufactured and integrated NAGICS can be achieved with modest demands on opto-mechanical precision.

The construction of a NAGICS prototype is scheduled and will allow for a proof of concept and the measurement of high-resolution transmission spectra of multi-species gas mixtures.

\footnotetext{
${ }^{1}$ Based on the $42 \mathrm{~m}$ E-ELT optical design report, E-TRE-ESO-227-138, iss. 3, with subsequent revsion to f/17.7 for the Nasmyth foci.

${ }^{2}$ Plus two unavoidable (refractive) flat optical windows for the captive gas vessel.
} 


\section{References}

Abrams, M. C., Davis, S. P., Rao, M. L. P., Engleman, Jr., R. \& Brault, J. W. (1994) High-resolution Fourier transform spectroscopy of the Meinel system of OH. ApJS, 93, 351-395. 88

Anglada-Escudé, G., Plavchan, P., Mills, S., Gao, P., García-Berríos, E., Lewis, N. S., Sung, K., Ciardi, D. ET AL. (2012) Design and Construction of Absorption Cells for Precision Radial Velocities in the K Band Using Methane Isotopologues. PASP, 124, 586-597. 96, 97, 100, 101, 171, 172, 173

BALONA, L. A. (2010) Wavelength calibration of arc spectra using intensity modelling. $M N$ RAS, 409, 1601-1605. 87

Baraffe, I., Chabrier, G., Allard, F. \& Hauschildt, P. H. (1998) Evolutionary models for solar metallicity low-mass stars: mass-magnitude relationships and color-magnitude diagrams. A\&A, 337, 403-412. 5, 30, 31

Baraffe, I., Chabrier, G., Allard, F. \& Hauschildt, P. H. (2002) Evolutionary models for low-mass stars and brown dwarfs: Uncertainties and limits at very young ages. A $\mathscr{E} A, \mathbf{3 8 2}, 563-572.30,31$

Barnes, S. A. (2003) On the Rotational Evolution of Solar- and Late-Type Stars, Its Magnetic Origins, and the Possibility of Stellar Gyrochronology. ApJ, 586, 464-479. 5, 6

Barrado y Navascues, D. \& Stauffer, J. R. (1996) Lithium abundance in binaries of the Hyades open cluster. A\& A, 310, 879-892. 34, 37

Basri, G., Mohanty, S., Allard, F., Hauschildt, P. H., Delfosse, X., Martín, E. L., Forveille, T. \& Goldman, B. (2000) An Effective Temperature Scale for Late$\mathrm{M}$ and L Dwarfs, from Resonance Absorption Lines of Cs I and Rb I. ApJ, 538, 363-385. 29

Bean, J. L., Seifahrt, A., Hartman, H., Nilsson, H., Wiedemann, G., Reiners, A., Dreizler, S. \& Henry, T. J. (2010) The CRIRES Search for Planets Around the Lowest-mass Stars. I. High-precision Near-infrared Radial Velocities with an Ammonia Gas Cell. ApJ, 713, 410-422. 95, 99, 100, 108, 171

Beckers, J. M. (1976) Reliability of sunspots as tracers of solar surface rotation. Nature, 260, 227-229. 93

Bender, C. F. \& Simon, M. (2008) The Detection of Low-Mass Companions in Hyades Cluster Spectroscopic Binary Stars. ApJ, 689, 416-429. 24, 34, 37, 43, 46

Bessell, M. S. \& Brett, J. M. (1988) JHKLM photometry - Standard systems, passbands, and intrinsic colors. PASP, 100, 1134-1151. 12, 22 
Bessell, M. S. \& Weis, E. W. (1987) The Cousins and Kron VRI systems. PASP, 99, 642-644. 21

Bleach, J. N., Wood, J. H., Smalley, B. \& Catalán, M. S. (2002a) Echelle observations of chromospheric activity in post-common-envelope binaries. MNRAS, 335, 593-609. 35

Bleach, J. N., Wood, J. H., Smalley, B. \& Catalán, M. S. (2002b) The measurement of rotational broadening in post-common-envelope binaries. MNRAS, 336, 611-620. 35, $52,53,60,79$

Bouchy, F., Pepe, F. \& Queloz, D. (2001) Fundamental photon noise limit to radial velocity measurements. A\&A, 374, 733-739. 94, 108

Bouvier, J., Forestini, M. \& Allain, S. (1997) The angular momentum evolution of low-mass stars. A\&A, 326, 1023-1043. 5

Braje, D. A., Kirchner, M. S., Osterman, S., Fortier, T. \& Diddams, S. A. (2008) Astronomical spectrograph calibration with broad-spectrum frequency combs. European Physical Journal D, 48, 57-66. 89

Breckinridge, J. B., Pierce, A. K. \& Stoll, C. P. (1975) Thorium Comparison Spectrum. ApJS, 29, 97.85

Browning, M. K., Basri, G., Marcy, G. W., West, A. A. \& Zhang, J. (2010) Rotation and Magnetic Activity in a Sample of M-Dwarfs. AJ, 139, 504-518. 5, 26, 61

Bruno, P., Leone, F., Oliva, E., Gennari, S., Mochi, I. \& Origlia, L. (2006) The preslit system of GIANO-TNG. In Society of Photo-Optical Instrumentation Engineers (SPIE) Conference Series, vol. 6269 of Society of Photo-Optical Instrumentation Engineers (SPIE) Conference Series. 101

Butler, R. P., Marcy, G. W., Williams, E., McCarthy, C., Dosanjh, P. \& Vogt, S. S. (1996) Attaining Doppler Precision of $3 \mathrm{M} \mathrm{s}-1 . P A S P, \mathbf{1 0 8}, 500.93,98$

Campbell, B. \& Walker, G. A. H. (1979) Precision radial velocities with an absorption cell. PASP, 91, 540-545. 93, 97

Campbell, B., Walker, G. A. H. \& Yang, S. (1988) A search for substellar companions to solar-type stars. ApJ, 331, 902-921. 93, 97, 98

Carpenter, J. M. (2001) Color Transformations for the 2MASS Second Incremental Data Release. AJ, 121, 2851-2871. 22, 30

Chabrier, G. \& Baraffe, I. (1997) Structure and evolution of low-mass stars. A $\& A, \mathbf{3 2 7}$, 1039-1053. 6

Chabrier, G. \& KüKer, M. (2006) Large-scale $\alpha^{\wedge} 2$-dynamo in low-mass stars and brown dwarfs. $A \& A, 446,1027-1037.6$

Claudi, R. U., Bonanno, A., Leccia, S., Ventura, R., Desidera, S., Gratton, R., Cosentino, R., Paternò, L. Et AL. (2005) Asteroseismology of Procyon A with SARG at TNG. $A \mathscr{E} A, 429$, L17-L20. 97 
Clough, S. A., Iacono, M. J. \& Moncet, J.-L. (1992) Line-by-Line Calculations of Atmospheric Fluxes and Cooling Rates: Application to Water Vapor. J. Geophys. Res., 97, 15761. 171

Clough, S. A., Shephard, M. W., Mlawer, E. J., Delamere, J. S., Iacono, M. J., Cady-Pereira, K., Boukabara, S. \& Brown, P. D. (2005) Atmospheric radiative transfer modeling: a summary of the AER codes. J. Quant. Spec. Radiat. Transf., 91, 233-244. 171

Cochran, W. D. \& Hatzes, A. P. (1993) McDonald Observatory Planetary Search - A high precision stellar radial velocity survey for other planetary systems. In Planets Around Pulsars, edited by J. A. Phillips, S. E. Thorsett \& S. R. Kulkarni, vol. 36 of Astronomical Society of the Pacific Conference Series. 93

Cram, L. E. \& Mullan, D. J. (1979) Model chromospheres of flare stars. I - Balmer-line profiles. ApJ, 234, 579-587. 67

Cutri, R. M., Skrutskie, M. F., van Dyk, S., Beichman, C. A., Carpenter, J. M., Chester, T., Cambresy, L., Evans, T. et Al. (2003) 2MASS All-Sky Catalog of Point Sources (Cutri+ 2003). VizieR Online Data Catalog, 2246, 0-+. 9, 16, 19, 30

D'Amato, F., Viciani, S., Oliva, E., Origlia, L. \& Mochi, I. (2008) Characterization of the HCl-HBr-HI gas absorption cell for GIANO-TNG. In Society of Photo-Optical Instrumentation Engineers (SPIE) Conference Series, vol. 7014 of Society of Photo-Optical Instrumentation Engineers (SPIE) Conference Series. 97, 101, 102, 144

de Cuyper, J.-P. \& Hensberge, H. (1998) Wavelength calibration at moderately high resolution. A\&AS, 128, 409-416. 88

Dekker, H., D’Odorico, S., Kaufer, A., Delabre, B. \& Kotzlowski, H. (2000) Design, construction, and performance of UVES, the echelle spectrograph for the UT2 Kueyen Telescope at the ESO Paranal Observatory. In Society of Photo-Optical Instrumentation Engineers (SPIE) Conference Series, edited by M. Iye \& A. F. Moorwood, vol. 4008 of Society of Photo-Optical Instrumentation Engineers (SPIE) Conference Series. 11

Delabre, B., Moorwood, A. \& Monnet, G. (2000) CRIRES Optical Design Report. Tech. Report VLT-TRE-ESO-14500-2096, European Southern Observatory, KarlSchwarzschild-Str. 2, D-85748 Garching bei München, issue 2.0. 115, 123, 125, 126, 127, $147,150,152$

Delfosse, X., Forveille, T., Perrier, C. \& Mayor, M. (1998) Rotation and chromospheric activity in field $\mathrm{M}$ dwarfs. $A \mathscr{E} A, \mathbf{3 3 1}, 581-595.5,6$

Delorme, P., Collier Cameron, A., Hebb, L., Rostron, J., Lister, T. A., Norton, A. J., Pollacco, D. \& West, R. G. (2011) Stellar rotation in the Hyades and Praesepe: gyrochronology and braking time-scale. MNRAS, 413, 2218-2234. 7, 63, 64, 65, 66

Denissenkov, P. A., Pinsonneault, M., Terndrup, D. M. \& Newsham, G. (2010) Angular Momentum Transport in Solar-type Stars: Testing the Timescale for Core-Envelope Coupling. ApJ, 716, 1269-1287. 65

Dienickx, P. (1992) Optical performance of large ground-based telescopes. Journal of Modern Optics, 39, 569-588. 146 
Dierickx, P., Enard, D. \& Tarenghi, M. (1995) VLT Optics: design of telescope optics. Tech. Report VLT-TRE-ESO-10000-0526, European Southern Observatory, KarlSchwarzschild-Str. 2, D-85748 Garching bei München, issue 1.B. 111, 116, 146

Doerr, H.-P., Kentischer, T. J., Steinmetz, T., Probst, R. A., Franz, M., Holzwarth, R., Udem, T., Hänsch, T. W. et Al. (2012) Performance of a laser frequency comb calibration system with a high-resolution solar echelle spectrograph. In Society of Photo-Optical Instrumentation Engineers (SPIE) Conference Series, vol. 8450 of Society of Photo-Optical Instrumentation Engineers (SPIE) Conference Series. 89

Donati, J.-F., Morin, J., Petit, P., Delfosse, X., Forveille, T., Aurière, M., Cabanac, R., Dintrans, B. ET AL. (2008) Large-scale magnetic topologies of early M dwarfs. MNRAS, 390, 545-560. 6

Durney, B. R., De Young, D. S. \& Roxburgh, I. W. (1993) On the generation of the large-scale and turbulent magnetic fields in solar-type stars. Sol. Phys., 145, 207-225. 6

Ettlinger, E., Giordano, P. \& Schneermann, M. (1999) Performance of the VlT mirror coating unit. The Messenger, 97, 4-8. 156

Figueira, P., Pepe, F., Lovis, C. \& Mayor, M. (2010a) Evaluating the stability of atmospheric lines with HARPS. A $\mathscr{E} A$, 515, A106. 88

Figueira, P., Pepe, F., Melo, C. H. F., Santos, N. C., Lovis, C., Mayor, M., Queloz, D., Smette, A. ET AL. (2010b) Radial velocities with CRIRES. Pushing precision down to $5-10 \mathrm{~m} / \mathrm{s}$. A\&A, 511, A55. 88

Fleming, T. A., Giampapa, M. S., Schmitt, J. H. M. M. \& Bookbinder, J. A. (1993) Stellar coronae at the end of the main sequence - A ROSAT survey of the late M dwarfs. ApJ, 410, 387-392. 25

Follert, R., Anglada-Escudé, G., Piskunov, N. \& the CRIRES+ Consortium (2013) CRIRES+: The CRIRES upgrade project. Tech. report, European Southern Observatory, VLT-TRE-ESO-14500-5813. xxi, 109, 127, 142, 176

Giclas, H. L., Burnham, R. \& Thomas, N. G. (1962) A proper motion survey in the Hyades. Lowell Observatory Bulletin, 5, 257-280. 19

Gizis, J. \& ReId, I. N. (1995) Low-Mass Binaries in the Hyades. AJ, 110, 1248. 30, 37

Gizis, J. E., Reid, I. N. \& Hawley, S. L. (2002) The Palomar/MSU Nearby Star Spectroscopic Survey. III. Chromospheric Activity, M Dwarf Ages, and the Local Star Formation History. AJ, 123, 3356-3369. 6

Golimowski, D. A., Leggett, S. K., Marley, M. S., Fan, X., Geballe, T. R., Knapp, G. R., Vrba, F. J., Henden, A. A. et Al. (2004) L' and M' Photometry of Ultracool Dwarfs. AJ, 127, 3516-3536. 22, 79

Gray, D. F. (2005) The Observation and Analysis of Stellar Photospheres. 27, 28

Greene, T. P., Tokunaga, A. T., Toomey, D. W. \& Carr, J. B. (1993) Cshell: a high spectral resolution 1-5 um cryogenic echelle spectrograph for the IRTF. In Society of Photo-Optical Instrumentation Engineers (SPIE) Conference Series, edited by A. M. 
Fowler, vol. 1946 of Society of Photo-Optical Instrumentation Engineers (SPIE) Conference Series. 100

Griffin, R. F., Griffin, R. E. M., Gunn, J. E. \& Zimmerman, B. A. (1985) Spectroscopic orbits for 16 more binaries in the Hyades field. AJ, 90, 609-642. 33, 37, 60, 79

Griffin, R. F., Griffin, R. E. M., Gunn, J. E. \& Zimmerman, B. A. (1988) A radialvelocity survey of the Hyades. AJ, 96, 172-197. 34, 37

GüDEL, M. (2004) X-ray astronomy of stellar coronae. A\&A Rev., 12, 71-237. 5, 44

Guenther, E. W., Paulson, D. B., Cochran, W. D., Patience, J., Hatzes, A. P. \& Macintosh, B. (2005) Low-mass companions to Hyades stars. A $\mathscr{G} A, \mathbf{4 4 2}, 1031-1039$. $24,37,45,47$

Guisard, S., Noethe, L. \& Spyromilio, J. (2000) Performance of active optics at the VLT. In Society of Photo-Optical Instrumentation Engineers (SPIE) Conference Series, edited by P. Dierickx, vol. 4003 of Society of Photo-Optical Instrumentation Engineers (SPIE) Conference Series. 146

Hanson, R. B. (1975) A study of the motion, membership, and distance of the Hyades cluster. $A J, \mathbf{8 0}, 379-401$. 16, 39, 79

Hatzes, A. P., Cochran, W. D., Endl, M., Guenther, E. W., Saar, S. H., Walker, G. A. H., Yang, S., Hartmann, M. ET AL. (2006) Confirmation of the planet hypothesis for the long-period radial velocity variations of $\beta$ Geminorum. A\&\&A, 457, 335-341. 93

Hatzes, A. P., Cochran, W. D., Endl, M., McArthur, B., Paulson, D. B., Walker, G. A. H., Campbell, B. \& Yang, S. (2003) A Planetary Companion to $\gamma$ Cephei A. ApJ, 599, 1383-1394. 93

Hatzes, A. P., Cochran, W. D., Mcarthur, B., Baliunas, S. L., Walker, G. A. H., Campbell, B., Irwin, A. W., Yang, S. Et AL. (2000) Evidence for a Long-Period Planet Orbiting $\epsilon$ Eridani. ApJ, 544, L145-L148. 93

Hauschildt, P. H., Allard, F. \& Baron, E. (1999) The NextGen Model Atmosphere Grid for $3000<=T \_$ef $f<=10,000 \mathrm{~K}$. ApJ, 512, 377-385. 23

Hawley, S. L., Gizis, J. E. \& Reid, I. N. (1996) The Palomar/MSU Nearby Star Spectroscopic Survey.II.The Southern M Dwarfs and Investigation of Magnetic Activity. AJ, 112, 2799. 6

Hawley, S. L. \& Reid, I. N. (1994) Comparison of Chromospheric and Coronal Activity in the Hyades and Pleiades. In Bulletin of the American Astronomical Society, vol. 26 of Bulletin of the American Astronomical Society. 7, 62

Hawley, S. L., Reid, I. N., Gizis, J. E. \& Byrne, P. B. (1999) Chromospheric Activity in Low Mass Stars: Observational Results from Clusters and the Field. In Solar and Stellar Activity: Similarities and Differences, edited by C. J. Butler \& J. G. Doyle, vol. 158 of Astronomical Society of the Pacific Conference Series. 6, 7, 44, 48, 62, 63 
Hempelmann, A., Schmitt, J. H. M. M., Schultz, M., Ruediger, G. \& Stepien, K. (1995) Coronal X-ray emission and rotation of cool main-sequence stars. $A \mathscr{G} A, \mathbf{2 9 4}$, 515-524. 47

Herriott, D. R. \& Schulte, H. J. (1965) Folded optical delay lines. Appl. Opt., 4, 883. 102

Hewett, P. C., Warren, S. J., Leggett, S. K. \& Hodgkin, S. T. (2006) The UKIRT Infrared Deep Sky Survey ZY JHK photometric system: passbands and synthetic colours. MNRAS, 367, 454-468. 31

Hoard, D. W., Wachter, S., Sturch, L. K., Widhalm, A. M., Weiler, K. P., Pretorius, M. L., Wellhouse, J. W. \& Gibiansky, M. (2007) Cool Companions to White Dwarf Stars from the Two Micron All Sky Survey All Sky Data Release. AJ, 134, 26-42. 36

Hunng, X., Schwenke, D. W. \& Lee, T. J. (2008) An accurate global potential energy surface, dipole moment surface, and rovibrational frequencies for $\mathrm{NH}_{3}$. J. Chem. Phys., 129(21), 214304. 100

Ihle, G. (2012) Interface Specification for Visiting Instruments in La Silla Observatory. Tech. Report LSO-SPE-ESO-00000-0003, La Silla Paranal Observatory, ESO, issue 2. 187

Irwin, J., Aigrain, S., Bouvier, J., Hebb, L., Hodgkin, S., Irwin, M. \& Moraux, E. (2009) The Monitor project: rotation periods of low-mass stars in M50. MNRAS, 392, 1456-1466. 63, 64

Irwin, J., Berta, Z. K., Burke, C. J., Charbonneau, D., Nutzman, P., West, A. A. \& FALCO, E. E. (2011) On the Angular Momentum Evolution of Fully Convective Stars: Rotation Periods for Field M-dwarfs from the MEarth Transit Survey. ApJ, 727, 56. 5

Ivanov, G. A. (2008) Catalogue of stars with high-proper motions. V2. (Ivanov, 2008). VizieR Online Data Catalog, 1306, 0-+. 9

JaCkson, R. J. \& JefFries, R. D. (2010a) Are the spin axes of stars randomly aligned within a cluster? MNRAS, 402, 1380-1390. 64

Jackson, R. J. \& Jeffries, R. D. (2010b) Chromospheric activity among fast-rotating M dwarfs in the open cluster NGC2516. MNRAS, 407, 465-478. 63

Kaufer, A. \& PAsquini, L. (1998) FEROS: the new fiber-linked echelle spectrograph for the ESO 1.52-m telescope. In Society of Photo-Optical Instrumentation Engineers (SPIE) Conference Series, edited by S. D'Odorico, vol. 3355 of Presented at the Society of PhotoOptical Instrumentation Engineers (SPIE) Conference. 11

Käufl, H. U., Amico, P., Ballester, P., Bendek, E., Biereichel, P., Bristow, P., Casali, M., Delabre, B. et al. (2006a) Good Vibrations: Report from the Commissioning of CRIRES. The Messenger, 126, 32-36. 108

Käufl, H. U., Amico, P., Ballester, P., Bendek Selman, E. A., Bristow, P., Casali, M., Delabre, B., Dobrzycka, D. Et Al. (2008) CRIRES: commissioning and first science results. In Society of Photo-Optical Instrumentation Engineers (SPIE) 
Conference Series, vol. 7014 of Society of Photo-Optical Instrumentation Engineers (SPIE) Conference Series. 108

Käufl, H.-U., Ballester, P., Biereichel, P., Delabre, B., Donaldson, R., Dorn, R., Fedrigo, E., Finger, G. ET Al. (2004) CRIRES: a high-resolution infrared spectrograph for ESO's VLT. In Society of Photo-Optical Instrumentation Engineers (SPIE) Conference Series, edited by A. F. M. Moorwood \& M. Iye, vol. 5492 of Society of PhotoOptical Instrumentation Engineers (SPIE) Conference Series. 89, 108, 109, 110

Käufl, H. U., Esteves, R., Fedrigo, E., Gojak, D., Lizon, J.-L., Marchetti, E., Oberti, S., Paufique, J. et Al. (2006b) CRIRES: Commissioning of the MACAO Adaptive Optics Module and General Status Report. The Messenger, 124, 2. 108

Kawaler, S. D. (1988) Angular momentum loss in low-mass stars. ApJ, 333, 236-247. 5

Kenyon, S. J. \& Hartmann, L. (1995) Pre-Main-Sequence Evolution in the Taurus-Auriga Molecular Cloud. ApJS, 101, 117-+. 22, 23, 79

Kerber, F., Nave, G. \& Sansonetti, C. J. (2008) The Spectrum of Th-Ar Hollow Cathode Lamps in the 691-5804 nm region: Establishing Wavelength Standards for the Calibration of Infrared Spectrographs. ApJS, 178, 374-381. 86, 88, 94

Kerber, F., Nave, G., Sansonetti, C. J. \& Bristow, P. (2009) From laboratory to the sky: Th-Ar wavelength standards for the cryogenic infrared echelle spectrograph (CRIRES). Physica Scripta Volume T, 134(1), 014007. 88

Kiraga, M. \& Stepien, K. (2007) Age-Rotation-Activity Relations for M Dwarf Stars. Acta Astron., 57, 149-172. 6

Kirkpatrick, J. D., Henry, T. J. \& McCarthy, Jr., D. W. (1991) A standard stellar spectral sequence in the red/near-infrared - Classes K5 to M9. ApJS, 77, 417-440. 27

Koch, A. \& Woenl, H. (1984) The use of molecular iodine absorption lines as wavelength references for solar Doppler shift measurements. A\&A, 134, 134-138. 93

Konopacky, Q. M., Ghez, A. M., Barman, T. S., Rice, E. L., Bailey, III, J. I., White, R. J., McLean, I. S. \& Duchêne, G. (2010) High-precision Dynamical Masses of Very Low Mass Binaries. ApJ, 711, 1087-1122. 37

Kraft, R. P. (1970) Stellar Rotation. p. 385. 6

Lamm, M. H., Mundt, R., Bailer-Jones, C. A. L. \& Herbst, W. (2005) Rotational evolution of low mass stars: The case of NGC 2264. A\&A, 430, 1005-1026. 63

Lanning, H. H. \& Pesch, P. (1981) HZ 9 - A white-dwarf-red-dwarf spectroscopic binary in the Hyades. ApJ, 244, 280-285. 35, 37

Lawrence, A., Warren, S. J., Almaini, O., Edge, A. C., Hambly, N. C., Jameson, R. F., Lucas, P., Casali, M. et al. (2012) UKIDSS-DR8 LAS, GCS and DXS Surveys (Lawrence+ 2012). VizieR Online Data Catalog, 2314, 0. 31

Leggett, S. K., Allard, F., Dahn, C., Hauschildt, P. H., Kerr, T. H. \& Rayner, J. (2000) Spectral Energy Distributions for Disk and Halo M Dwarfs. ApJ, 535, 965-974. 22 
lo Curto, G., Manescau, A., Avila, G., Pasquini, L., Wilken, T., Steinmetz, T., Holzwarth, R., Probst, R. et Al. (2012) Achieving a few $\mathrm{cm} / \mathrm{sec}$ calibration repeatability for high resolution spectrographs: the laser frequency comb on HARPS. In Society of Photo-Optical Instrumentation Engineers (SPIE) Conference Series, vol. 8446 of Society of Photo-Optical Instrumentation Engineers (SPIE) Conference Series. 89

López-Santiago, J., Montes, D., Gálvez-Ortiz, M. C., Crespo-Chacón, I., Martínez-Arnáiz, R. M., Fernández-Figueroa, M. J., de Castro, E. \& Cornide, M. (2010) A high-resolution spectroscopic survey of late-type stars: chromospheric activity, rotation, kinematics, and age. $A \mathscr{E} A, \mathbf{5 1 4}$, A97. 26

Lovis, C. \& PePe, F. (2007) A new list of thorium and argon spectral lines in the visible. $A \mathscr{E} A, 468,1115-1121.86,88,94$

LuYten, W. J. (1979) NLTT catalogue. Volume_I. +90_to_+30_. Volume._II. +30_to_0_. 19

MacGregor, K. B. \& Brenner, M. (1991) Rotational evolution of solar-type stars. I Main-sequence evolution. ApJ, 376, 204-213. 5

Mahadevan, S. \& Ge, J. (2009) The Use of Absorption Cells as a Wavelength Reference for Precision Radial Velocity Measurements in the Near-Infrared. ApJ, 692, 1590-1596. 98, 99

Mahajan, V. (1998) Optical Imaging and Aberrations: Ray geometrical optics. No. pts. 1-2 in SPIE Press monograph, SPIE Optical Engineering Press, URL http://books.google.de/books?id=I_1AAQAAIAAJ. 184

Marcy, G. W. \& Butler, R. P. (1992) Precision radial velocities with an iodine absorption cell. PASP, 104, 270-277. 93, 98

Marcy, G. W. \& Chen, G. H. (1992) The rotation of M dwarfs. ApJ, 390, 550-559. 5

Mayor, M., Pepe, F., Queloz, D., Bouchy, F., Rupprecht, G., Lo Curto, G., Avila, G., Benz, W. et AL. (2003) Setting New Standards with HARPS. The Messenger, 114, 20-24. 90

Mayor, M. \& Queloz, D. (1995) A Jupiter-mass companion to a solar-type star. Nature, 378, 355-359. 93

Mermilliod, J.-C., Mayor, M. \& Udry, S. (2009) Catalogues of radial and rotational velocities of $1253 \mathrm{~F}-\mathrm{K}$ dwarfs in 13 nearby open clusters. A\&A, 498, 949-960. 60, 79

Mestel, L. (1984) Angular Momentum Loss During Pre-Main Sequence Contraction. In Cool Stars, Stellar Systems, and the Sun, edited by S. L. Baliunas \& L. Hartmann, vol. 193 of Lecture Notes in Physics, Berlin Springer Verlag. 5

Mickelson, M. E., Steyert, D. W., Sirota, J. M. \& Reuter, D. C. (2001) Cooled Multi-pass Cells for Visible and Infrared Spectroscopy. In AAS/Division for Planetary Sciences Meeting Abstracts \#33, vol. 33 of Bulletin of the American Astronomical Society. 88 
Mohanty, S. \& Basri, G. (2003) Rotation and Activity in Mid-M to L Field Dwarfs. ApJ, 583, 451-472. 5, 6, 29

Morin, J., Donati, J.-F., Petit, P., Delfosse, X., Forveille, T. \& Jardine, M. M. (2010) Large-scale magnetic topologies of late $\mathrm{M}$ dwarfs. MNRAS, 407, 2269-2286. 6

Murphy, M. T., Locke, C. R., Light, P. S., Luiten, A. N. \& Lawrence, J. S. (2012) Laser frequency comb techniques for precise astronomical spectroscopy. MNRAS, 422, 761-771. 89

Murphy, M. T., Tzanavaris, P., Webb, J. K. \& Lovis, C. (2007a) Selection of ThAr lines for wavelength calibration of echelle spectra and implications for variations in the fine-structure constant. MNRAS, 378, 221-230. 87

Murphy, M. T., Udem, T., Holzwarth, R., Sizmann, A., Pasquini, L., AraujoHauck, C., Dekker, H., D'Odorico, S. et Al. (2007b) High-precision wavelength calibration of astronomical spectrographs with laser frequency combs. MNRAS, 380, 839847. 89

Nai-Cheng, S., Yao-Xiang, W., Yi-Min, S., Cheng-Yang, L., Xue-Bin, Z. \& Chu, W. (1981) Iodine and Methane Stabilized He-Ne Lasers as Wavelength Standards. In Precision Measurement and Fundamental Constants, edited by B. N. Taylor \& W. D. Phillips. 100

Nordström, B., Mayor, M., Andersen, J., Holmberg, J., Pont, F., Jørgensen, B. R., Olsen, E. H., Udry, S. ET AL. (2004) The Geneva-Copenhagen survey of the Solar neighbourhood. Ages, metallicities, and kinematic properties of $\sim 14000 \mathrm{~F}$ and $\mathrm{G}$ dwarfs. A\&A A 418, 989-1019. 26

Noyes, R. W., Hartmann, L. W., Baliunas, S. L., Duncan, D. K. \& Vaughan, A. H. (1984) Rotation, convection, and magnetic activity in lower main-sequence stars. ApJ, 279, 763-777. 6

O’Dwyer, I. J., Chu, Y.-H., Gruendl, R. A., Guerrero, M. A. \& Webbink, R. F. (2003) Hard X-Ray Emission Associated with White Dwarfs. AJ, 125, 2239-2254. 35

Oliva, E., Hatzes, A., Piskunov, N., Reiners, A., Käufl, H. U., Ferruzzi, D., TozzI, A. \& OrIGLIA, L. (2012) Upgrading CRIRES-VLT to cross-dispersed mode. In Society of Photo-Optical Instrumentation Engineers (SPIE) Conference Series, vol. 8446 of Society of Photo-Optical Instrumentation Engineers (SPIE) Conference Series. 109

Ossendrijver, M. (2003) The solar dynamo. A\&AA Rev., 11, 287-367. 6

Osterman, S., Diddams, S., Beasley, M., Froning, C., Hollberg, L., Macqueen, P., Mbele, V. \& Weiner, A. (2007) A proposed laser frequency comb-based wavelength reference for high-resolution spectroscopy. In Society of Photo-Optical Instrumentation Engineers (SPIE) Conference Series, vol. 6693 of Society of Photo-Optical Instrumentation Engineers (SPIE) Conference Series. 89

Palmer, B. A. \& Engleman, R. (1983) Atlas of the Thorium spectrum. 87, 88 
Paufique, J., Biereichel, P., Donaldson, R., Delabre, B., Fedrigo, E., Franza, F., Gigan, P., Gojak, D. ET AL. (2006) MACAO-CRIRES, a step towards highresolution spectroscopy. ArXiv Astrophysics e-prints. 108, 115

Pels, G., Oort, J. H. \& Pels-Kluyver, H. A. (1975) New members of the Hyades cluster and a discussion of its structure. $A \mathscr{E} A, \mathbf{4 3}, 423-441.19$

Perryman, M. A. C., Brown, A. G. A., Lebreton, Y., Gomez, A., Turon, C., Cayrel de Strobel, G., Mermilliod, J. C., Robichon, N. et Al. (1998) The Hyades: distance, structure, dynamics, and age. A\& A, 331, 81-120. 7, 25, 27, 30

Phillips, D. F., Glenday, A. G., Li, C.-H., Cramer, C., Furesz, G., Chang, G., Benedick, A. J., Chen, L.-J. ET AL. (2012) Calibration of an astrophysical spectrograph below $1 \mathrm{~m} / \mathrm{s}$ using a laser frequency comb. Optics Express, 20, 13711-13726. 89

Pizzolato, N., Maggio, A., Micela, G., Sciortino, S. \& Ventura, P. (2003) The stellar activity-rotation relationship revisited: Dependence of saturated and non-saturated $\mathrm{X}$-ray emission regimes on stellar mass for late-type dwarfs. A\&A, 397, 147-157. 6, 47, 53

Prosser, C. F., Shetrone, M. D., Dasgupta, A., Backman, D. E., Laaksonen, B. D., Baker, S. W., Marschall, L. A., Whitney, B. A. et Al. (1995) Rotation periods of open-cluster stars, 3. PASP, 107, 211-218. 64, 66

Pye, J. P., Hodgkin, S. T., Stern, R. A. \& Stauffer, J. R. (1994) ROSAT X-ray luminosity functions of the Hyades dK and dM stars. MNRAS, 266, 798. 24

Quinlan, F., Ycas, G., Osterman, S. \& Diddams, S. A. (2010) A 12.5 GHz-spaced optical frequency comb spanning $>400 \mathrm{~nm}$ for near-infrared astronomical spectrograph calibration. Review of Scientific Instruments, 81(6), 063105. 89

Radick, R. R., Thompson, D. T., Lockwood, G. W., Duncan, D. K. \& Baggett, W. E. (1987) The activity, variability, and rotation of lower main-sequence Hyades stars. ApJ, 321, 459-472. 7, 9, 42, 60, 64, 66, 79

Rebassa-Mansergas, A., Schreiber, M. R. \& Gaensicke, B. T. (2012) M dwarf companions to white dwarfs I: relating magnetic activity, rotation and age. ArXiv e-prints. 36, 66

Rebolo, R. \& Beckman, J. E. (1988) Rotation, Activity and Lithium Depletion in the Hyades Late Main Sequence. In The Impact of Very High $S / N$ Spectroscopy on Stellar Physics, edited by G. Cayrel de Strobel \& M. Spite, vol. 132 of IAU Symposium. 7

Redman, S. L., Kerber, F., Nave, G., Mahadevan, S., Ramsey, L. W., Smoker, J., Käufl, H.-U. \& FigueirA, P. R. L. (2012) Near-infrared calibration systems for precise radial-velocity measurements. In Society of Photo-Optical Instrumentation Engineers (SPIE) Conference Series, vol. 8446 of Society of Photo-Optical Instrumentation Engineers (SPIE) Conference Series. 94

Reid, I. N. \& Gizis, J. E. (1997) Low-Mass Binaries in the Hyades: A Scarcity of Brown Dwarfs. AJ, 114, 1992. 37, 38 
Reid, I. N. \& MAhoney, S. (2000) Low-mass spectroscopic binaries in the Hyades: a candidate brown dwarf companion. MNRAS, 316, 827-844. 7, 9, 29, 37, 38, 42, 49, 50, 54, 79

ReID, N. (1992) Proper motions from Schmidt plates. II - The Hyades. MNRAS, 257, 257302. $9,10,19,21$

REID, N. (1993) Low-Mass Stars in the Hyades. MNRAS, 265, 785. 21, 31

Reid, N., Hawley, S. L. \& Mateo, M. (1995) Chromospheric and coronal activity in low-mass Hyades dwarfs. MNRAS, 272, 828-842. 7, 24, 25

ReINERs, A. (2007) The narrowest M-dwarf line profiles and the rotation-activity connection at very slow rotation. A\&BA, 467, 259-268. 26

Reiners, A. \& Basri, G. (2007) The First Direct Measurements of Surface Magnetic Fields on Very Low Mass Stars. ApJ, 656, 1121-1135. 6

Reiners, A. \& BAsri, G. (2008) Chromospheric Activity, Rotation, and Rotational Braking in $\mathrm{M}$ and L Dwarfs. ApJ, 684, 1390-1403. 6, 41

Reiners, A. \& Basri, G. (2010) A Volume-Limited Sample of 63 M7-M9.5 Dwarfs. II. Activity, Magnetism, and the Fade of the Rotation-Dominated Dynamo. ApJ, 710, 924935. $6,61,62$

Reiners, A., Basri, G. \& Browning, M. (2009) Evidence for Magnetic Flux Saturation in Rapidly Rotating M Stars. ApJ, 692, 538-545. 47

Reiners, A., Joshi, N. \& Goldman, B. (2012) A Catalog of Rotation and Activity in Early-M Stars. AJ, 143, 93. 5, 6, 26, 48, 61, 62, 63, 64

Reiners, A. \& Mohanty, S. (2012) Radius-dependent Angular Momentum Evolution in Low-mass Stars. I. ApJ, 746, 43. 6, 64, 67

ROSAT Consortium (2000) The Second ROSAT Source Catalog of Pointed Observations with the Position Sensitive Proportional Counter. In ROSAT News, vol. 72, ROSAT Consortium. 79

Röser, S., Schilbach, E., Piskunov, A. E., Kharchenko, N. V. \& Scholz, R.-D. (2011) A deep all-sky census of the Hyades. A\&A A 531, A92+. 9, 10, 16, 25, 30, 35, 39, 79

Rosman, K. J. R. \& Taylor, P. D. P. (1998) Isotopic Compositions of the Elements 1997. Journal of Physical and Chemical Reference Data, 27, 1275-1287. 87

Rothman, L. S., Gordon, I. E., Barbe, A., Benner, D. C., Bernath, P. F., Birk, M., Boudon, V., Brown, L. R. ET AL. (2009) The HITRAN 2008 molecular spectroscopic database. J. Quant. Spec. Radiat. Transf., 110, 533-572. 86, 108, 171

Rothman, L. S., Jacquemart, D., Barbe, A., Chris Benner, D., Birk, M., Brown, L. R., Carleer, M. R., Chackerian, C. et Al. (2005) The Hitran 2004 molecular spectroscopic database. J. Quant. Spec. Radiat. Transf., 96, 139-204. 171

Rousselot, P., Lidman, C., Cuby, J.-G., Moreels, G. \& Monnet, G. (2000) Nightsky spectral atlas of $\mathrm{OH}$ emission lines in the near-infrared. $A \mathscr{E} A, \mathbf{3 5 4}, 1134-1150.88$ 
Roxburgh, I. W. (1983) Stellar winds and spindown in solar type stars. In Solar and Stellar Magnetic Fields: Origins and Coronal Effects, edited by J. O. Stenflo, vol. 102 of IAU Symposium. 5

Rupprecht, G. (2005) Requirements for Scientific Instruments on the VLT Unit Telescopes. Tech. Report VLT-SPE-ESO-10000-2723, La Silla Paranal Observatory, ESO, issue 1. 111, 112,119

SaAR, S. H. \& Osten, R. A. (1997) Rotation, turbulence and evidence for magnetic fields in southern dwarfs. MNRAS, 284, 803-810. 26

Scholz, A. \& EislÖffel, J. (2007) The first rotation periods in Praesepe. MNRAS, 381, 1638-1646. 64, 65, 66

Scholz, A., Irwin, J., Bouvier, J., Sipőcz, B. M., Hodgkin, S. \& Eislöffel, J. (2011) Rotation periods for very low mass stars in Praesepe. MNRAS, 413, 2595-2605. 64, 65,66

Schreiber, M. R. \& Gänsicke, B. T. (2003) The age, life expectancy, and space density of Post Common Envelope Binaries. A\&A, 406, 305-321. 35, 37

SchriJver, C. J. \& ZwaAn, C. (2008) Solar and Stellar Magnetic Activity. 5, 53

Schwan, H. (1991) The distance and main sequence of the Hyades cluster based on 145 stars with highly accurate proper motions obtained from work on the catalogues FK 5 and PPM. A\&A, 243, 386-400. 16, 39, 79

Seemann, U., Reiners, A., Seifahrt, A. \& Kürster, M. (2011) The Activity and Rotation Limit in the Hyades. In 16th Cambridge Workshop on Cool Stars, Stellar Systems, and the Sun, edited by C. Johns-Krull, M. K. Browning \& A. A. West, vol. 448 of Astronomical Society of the Pacific Conference Series. xxi

Seifahrt, A., Käufl, H. U., ZÄngl, G., Bean, J. L., Richter, M. J. \& SiebenmorGEN, R. (2010) Synthesising, using, and correcting for telluric features in high-resolution astronomical spectra . A near-infrared case study using CRIRES. A\&A, 524, A11. 95, 100, 171

Seifahrt, A., Reiners, A., Scholz, A. \& Basri, G. (2009) Activity and rotation of low mass stars in young open clusters. In American Institute of Physics Conference Series, edited by E. Stempels, vol. 1094 of American Institute of Physics Conference Series. 42, 79

Siess, L., Dufour, E. \& Forestini, M. (2000) An internet server for pre-main sequence tracks of low- and intermediate-mass stars. A\&A, 358, 593-599. 6

Skumanich, A. (1972) Time Scales for CA II Emission Decay, Rotational Braking, and Lithium Depletion. ApJ, 171, 565-+. 5

Smith, W. J. (1991) Fundamentals of the optical tolerance budget. vol. 1354 of Society of Photo-Optical Instrumentation Engineers (SPIE) Conference Series, URL http://dx.doi.org/10.1117/12.47901.184 
Stauffer, J. R., Giampapa, M. S., Herbst, W., Vincent, J. M., Hartmann, L. W. \& Stern, R. A. (1991) The chromospheric activity of low-mass stars in the Hyades. ApJ, 374, 142-149. 48, 49, 50, 62

Stauffer, J. R., Hartmann, L. W. \& Latham, D. W. (1987) Rotational velocities of low-mass stars in the Hyades. ApJ, 320, L51-L55. 7, 9, 38, 39, 42, 60, 79

Stauffer, J. R., Hartmann, L. W., Prosser, C. F., Randich, S., Balachandran, S., Patten, B. M., Simon, T. \& Giampapa, M. (1997a) Rotational Velocities and Chromospheric/Coronal Activity of Low-Mass Stars in the Young Open Clusters IC 2391 and IC 2602. ApJ, 479, 776-+. 7, 9, 21, 24, 37, 38, 42, 49, 50, 52, 54, 60, 79

Stauffer, J. R., Hartmann, L. W., Prosser, C. F., Randich, S., Balachandran, S., Patten, B. M., Simon, T. \& Giampapa, M. (1997b) Rotational Velocities and Chromospheric/Coronal Activity of Low-Mass Stars in the Young Open Clusters IC 2391 and IC 2602. ApJ, 479, 776. 63

Stauffer, J. R., Liebert, J., Giampapa, M., Macintosh, B., Reid, N. \& Hamilton, D. (1994) Radial velocities of very low mass stars and candidate brown dwarf members of the Hyades and Pleiades. AJ, 108, 160-174. 48

Steinmetz, T., Wilken, T., Araujo-Hauck, C., Holzwarth, R., Hänsch, T. W., Pasquini, L., Manescau, A., D’Odorico, S. Et AL. (2008) Laser Frequency Combs for Astronomical Observations. Science, 321, 1335-. 89

Stern, R. A., Schmitt, J. H. M. M. \& Kahabka, P. T. (1995) ROSAT All-Sky Survey Observations of the Hyades Cluster. ApJ, 448, 683-+. 10, 24, 25, 35, 79

Stern, R. A., Schmitt, J. H. M. M., Pye, J. P., Hodgkin, S. T., Stauffer, J. R. \& Simon, T. (1994) Coronal X-ray sources in the Hyades: A 40 kilosecond ROSAT pointing. ApJ, 427, 808-821. 24, 25

Stoehr, F., Fraquelli, D., Kamp, I., Kimball, T., Levay, K., Rogers, T., Smith, M., Thompson, R. et AL. (2007) DER_SNR: A Simple \& General Spectroscopic Signalto-Noise Measurement Algorithm. Space Telescope European Coordinating Facility Newsletter, 42, 4-+. 19

Swann, W. C. \& Gilbert, S. L. (2000) Pressure-induced shift and broadening of 15101540$\mathrm{nm}$ acetylene wavelength calibration lines. Journal of the Optical Society of America B Optical Physics, 17, 1263-1270. 98

Swann, W. C. \& Gilbert, S. L. (2002) Pressure-induced shift and broadening of 15601630$\mathrm{nm}$ carbon monoxide wavelength-calibration lines. Journal of the Optical Society of America B Optical Physics, 19, 2461-2467. 98

Swann, W. C. \& Gilbert, S. L. (2005) Line centers, pressure shift, and pressure broadening of 1530-1560 nm hydrogen cyanide wavelength calibration lines. Journal of the Optical Society of America B Optical Physics, 22, 1749-1756. 98

Tokunaga, A. T. (2000) Infrared Astronomy. pp. 143-+. 9, 22, 23 
Urban, Š., Tu, N., Narahari Rao, K. \& Guelachvili, G. (1989) Analysis of highresolution Fourier transform spectra of ${ }^{14} \mathrm{NH}_{3}$ at $2.3 \mu \mathrm{m}$. Journal of Molecular Spectroscopy, 133, 312-330. 100

Valdivielso, L., Esparza, P., Martín, E. L., Maukonen, D. \& Peale, R. E. (2010) A New Gas Cell for High-precision Doppler Measurements in the Near-infrared. ApJ, 715, 1366-1369. 101

Valenti, J. A., Butler, R. P. \& Marcy, G. W. (1995) Determining Spectrometer Instrumental Profiles Using FTS Reference Spectra. PASP, 107, 966. 98

van Altena, W. F. (1966) Low-luminosity members of the Hyades cluster. AJ, 71, 482-512. 19

van Bueren, H. G. (1952) On the structure of the Hyades cluster. Bull. Astron. Inst. Netherlands, 11, 385-+. 19

Voges, W., Aschenbach, B., Boller, T., Bräuninger, H., Briel, U., Burkert, W., Dennerl, K., Englhauser, J. Et al. (1999) The ROSAT all-sky survey bright source catalogue. A\&A A, 349, 389-405. 46, 79

Voges, W., Aschenbach, B., Boller, T., Brauninger, H., Briel, U., Burkert, W., Dennerl, K., Englhauser, J. Et Al. (2000) ROSAT all-sky survey faint source catalogue. IAU Circ., 7432, 1. 79

Vogt, S. S. (1987) The Lick Observatory Hamilton Echelle Spectrometer. PASP, 99, 12141228. 98

Walker, G. A. H., Bohlender, D. A., Walker, A. R., Irwin, A. W., Yang, S. L. S. \& Larson, A. (1992) Gamma Cephei - Rotation or planetary companion? ApJ, 396, L91-L94. 93

Walker, G. A. H., Walker, A. R., Irwin, A. W., Larson, A. M., Yang, S. L. S. \& Richardson, D. C. (1995) A search for Jupiter-mass companions to nearby stars. Icarus, 116, 359-375. 93

Walkowicz, L. M. \& Hawley, S. L. (2009) Tracers of Chromospheric Structure. I. Observations of $\mathrm{Ca}$ II $\mathrm{K}$ and $\mathrm{H} \alpha$ in M Dwarfs. AJ, 137, 3297-3313. 56, 67

Wallace, L., Hinkle, K. H., Livingston, W. C. \& Davis, S. P. (2011) An Optical and Near-infrared (2958-9250 A) Solar Flux Atlas. ApJS, 195, 6. 27, 35

West, A. A. \& Basri, G. (2009a) A First Look at Rotation in Inactive Late-Type M Dwarfs. ApJ, 693, 1283-1289. 26

West, A. A. \& Basri, G. (2009b) A First Look at Rotation in Inactive Late-Type M Dwarfs. ApJ, 693, 1283-1289. 61

West, A. A., Hawley, S. L., Bochanski, J. J., Covey, K. R., Reid, I. N., Dhital, S., Hilton, E. J. \& Masuda, M. (2008) Constraining the Age-Activity Relation for Cool Stars: The Sloan Digital Sky Survey Data Release 5 Low-Mass Star Spectroscopic Sample. AJ, 135, 785-795. 6, 48, 63 
West, A. A., Hawley, S. L., Walkowicz, L. M., Covey, K. R., Silvestri, N. M., Raymond, S. N., Harris, H. C., Munn, J. A. et Al. (2004) Spectroscopic Properties of Cool Stars in the Sloan Digital Sky Survey: An Analysis of Magnetic Activity and a Search for Subdwarfs. AJ, 128, 426-436. 6, 48

White, J. U. (1942) Long optical paths of large aperture. Journal of the Optical Society of America (1917-1983), 32, 285. 102

Worthey, G. \& Lee, H.-C. (2011) An Empirical UBV RI JHK Color-Temperature Calibration for Stars. ApJS, 193,1-+. 22, 23

Wright, N. J., Drake, J. J., Mamajek, E. E. \& Henry, G. W. (2011) The Stellaractivity-Rotation Relationship and the Evolution of Stellar Dynamos. ApJ, 743, 48. 6, 37

Wybourne, B. G. \& Meggers, W. F. (1965) Spectroscopic Properties of Rare Earths. Physics Today, 18, 70. 87

Youngworth, R. N. (2006) Twenty-first century optical tolerancing: a look at the past and improvements for the future. vol. 6342 of Society of Photo-Optical Instrumentation Engineers (SPIE) Conference Series, URL http://dx.doi.org/10.1117/12.692188. 184

Yurchenko, S. N., Zheng, J., Lin, H., Jensen, P. \& Thiel, W. (2005) Potentialenergy surface for the electronic ground state of $\mathrm{NH}_{3}$ up to $20000 \mathrm{~cm}^{-1}$ above equilibrium. J. Chem. Phys., 123(13), 134308. 100

Zacharias, N., Finch, C., Girard, T., Hambly, N., Wycoff, G., Zacharias, M. I., Castillo, D., Corbin, T. ET Al. (2010) The Third US Naval Observatory CCD Astrograph Catalog (UCAC3). AJ, 139, 2184-2199. 9

Zacharias, N., Monet, D. G., Levine, S. E., Urban, S. E., Gaume, R. \& Wycoff, G. L. (2004a) The Naval Observatory Merged Astrometric Dataset (NOMAD). In American Astronomical Society Meeting Abstracts, vol. 36 of Bulletin of the American Astronomical Society. 9, 16

Zacharias, N., Urban, S. E., Zacharias, M. I., Wycoff, G. L., Hall, D. M., Monet, D. G. \& Rafferty, T. J. (2004b) The Second US Naval Observatory CCD Astrograph Catalog (UCAC2). AJ, 127, 3043-3059. 9 



\section{List of publications}

Chen, G., van Boekel, R., Wang, H., Nikolov, N., Seemann, U., and Henning, Th.: 2014, Astron. Astroph. 567, AA8.

Chen, G., van Boekel, R., Madhusudhan, N., Wang, H., Nikolov, N., Seemann, U., and Henning, Th.: 2014, Astron. Astroph. 564, AA6.

Sarmiento, L. F., Reiners, A., Seemann, U., et al. 2014, Proc. SPIE, 9147, 914754

Seemann, U., Anglada-Escude, G., Baade, D., et al. 2014, Proc. SPIE, 9147, 91475G

Shulyak, D., Reiners, A., Seemann, U., Kochukhov, O., Piskunov, N. 2014. Magnetic fields in M-dwarfs from high-resolution infrared spectroscopy. IAU Symposium 302, 170-173.

Chen, G., van Boekel, R., Wang, H., Nikolov, N., Fortney, J. J., Seemann, U., Wang, W., Mancini, L., and Henning, Th.: 2013, Astron. Astroph. 563, A40.

Glauser, A.M., van Boekel, R., Krause, O., Henning, T., Benneke, B., Bouwman, J., Cubillos, P.E., Crossfield, I.J.M., Detre, Ö.H., Ebert, M., Grözinger, U., Güdel, M., Harrington, J., Justtanont, K., Klaas, U., Lenzen, R., Madhusudhan, N., Meyer, M.R., Mordasini, C., Müller, F., Ottensamer, R., Plesseria, J.-Y., Quanz, S.P., Reiners, A., Renotte, E., Rohloff, R.-R., Scheithauer, S., Schmid, H.M., Schrader, J.-R., Seemann, U., Stam, D., Vandenbussche, B., and Wehmeier, U.: 2013, (ArXiv e-prints, arXiv:1305.3089) Journal of Astronomical Instrumentation, 2, 50004.

Müller, A., Roccatagliata, V., Henning, T., Fedele, D., Pasquali, A., Caffau, E., RodríguezLedesma, M.V., Mohler-Fischer, M., Seemann, U., and Klement, R.J.: 2013, Astron. Astroph. 556, A3.

Seemann, U., Reiners, A., Seifahrt, A., and Kürster, M.: 2013, Astron. Astroph. (submitted).

Shulyak, D., Reiners, A., Seemann, U., Kochukhov, O., Piskunov, N.: 2014, Astron. Astroph. 563, A35.

Adami, C., Jouvel, S., Guennou, L., Le Brun, V., Durret, F., Clement, B., Clerc, N., Comerón, S., Ilbert, O., Lin, Y., Russeil, D., and Seemann, U.: 2012, Astron. Astroph. 540, A105.

Lebzelter, T., Seifahrt, A., Uttenthaler, S., Ramsay, S., Hartman, H., Nieva, M.-F., Przybilla, N., Smette, A., Wahlgren, G.M., Wolff, B., Hussain, G.A.J., Käufl, H.U., and Seemann, U.: 2012, Astron. Astroph. 539, A109.

Mislis, D., Heller, R., Fernandez, J., Seemann, U., Ioannidis, P., and Avdellidou, C.: 2012, 10th Hellenic Astronomical Conference, 12.

Setiawan, J., Roccatagliata, V., Fedele, D., Henning, T., Pasquali, A., Rodríguez-Ledesma, M.V., Caffau, E., Seemann, U., and Klement, R.J.: 2012, Astron. Astroph. 540, A141. 
Lebzelter, T., Seifahrt, A., Ramsay, S., Almeida, P., Bagnulo, S., Hartman, H., Hussain, G., Käufl, H.-U., Nieva, M.F., Przybilla, N., Seemann, U., Smette, A., Uttenthaler, S., Wahlgren, G., and Wolff, B.: 2011, Why Galaxies Care about AGB Stars II: Shining Examples and Common Inhabitants 445, 623.

Seemann, U., Reiners, A., Seifahrt, A., and Kürster, M.: 2011, 16th Cambridge Workshop on Cool Stars, Stellar Systems, and the Sun 448, 313.

Ramsay, S.K., Lebzelter, T., Seifahrt, A., Almeida, P., Bagnulo, S., Hartman, H., Hussain, G., Käufl, H.U., Nieva, M.-F., Przybilla, N., Seemann, U., Smette, A., Uttenthaler, S., Wahlgren, G.M., and Wolff, B.: 2011, Bulletin de la Societe Royale des Sciences de Liege 80, 509 .

Lebzelter, T., Seifahrt, A., Ramsay, S., Almeida, P., Bagnulo, S., Dall, T., Hartman, H., Hussain, G., Käufl, H.U., Nieva, M.-F., Przybilla, N., Seemann, U., Smette, A., Uttenthaler, S., Wahlgren, G., and Wolff, B.: 2010, The Messenger 139, 33. 